FORSCHUNGSERGEBNISSE DER WIRTSCHAFTSUNIVERSITÄT WIEN

Matthias Fink

\title{
Erfolgsfaktor Selbstverpflichtung bei vertrauensbasierten Kooperationen
}


FORSCHUNGSERGEBNISSE DER

WIRTSCHAFTSUNIVERSITÄT WIEN

Matthias Fink

\section{Erfolgsfaktor Selbstverpflichtung bei vertrauensbasierten Kooperationen}

Durch seine verhaltensnormierende Wirkung stellt Vertrauen einen wirkungsvollen Mechanismus zur Koordination sozialer Beziehungen dar. Es stellt sich die Frage, ob dieser allgemeine Koordinationsmechanismus auch in den Bereichen der Gesellschaft erfolgreich seine Wirkung entfalten kann, in denen bisher vor allem der Marktmechanismus und hierarchische Strukturen für die Abstimmung der Transaktionsbeziehungen verantwortlich gemacht wurden. Der Autor zeigt für den betriebswirtschaftlichen Kontext der zwischenbetrieblichen Kooperation die Möglichkeit, Notwendigkeit und den Erfolg vertrauensbasierter Koordination auf. Die Aussagekraft der in dieser Arbeit präsentierten Argumentation erhält durch eine umfangreiche Befragung von Geschäftsführern kleiner und mittlerer Betriebe empirische Bestätigung.

Matthias Fink, geboren 1978 in Mödling bei Wien, studierte Handelswissenschaften und Betriebswirtschaftslehre an der Wirtschaftsuniversität Wien, wo er 2004 im Fachbereich Betriebswirtschaftslehre der Klein- und Mittelbetriebe promovierte. Derzeit ist er Universitätsassistent am Institut für Klein- und Mittelbetriebe an der Wirtschaftsuniversität Wien. Seine Forschungsschwerpunkte sind Transaktionstheorie, Kooperationsforschung, Koordinationsmechanismen interorganisationaler Transaktionsbeziehungen und Vertrauen im ökonomischen Kontext. 
Erfolgsfaktor Selbstverpflichtung bei vertrauensbasierten Kooperationen 


\section{Forschungsergebnisse der Wirtschaftsuniversität Wien}

\section{Band 6}

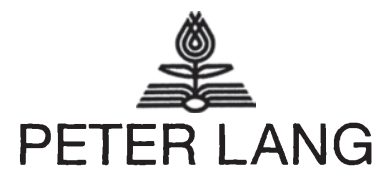

Frankfurt am Main · Berlin · Bern · Bruxelles · New York · Oxford · Wien 
Matthias Fink

\section{Erfolgsfaktor Selbstverpflichtung \\ bei vertrauensbasierten Kooperationen}

Mit einem empirischen Befund

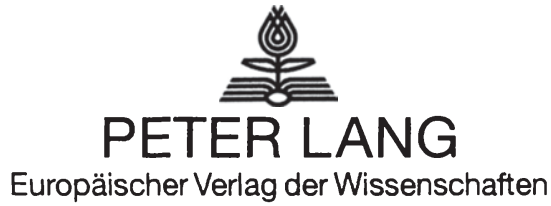


Bibliografische Information Der Deutschen Bibliothek Die Deutsche Bibliothek verzeichnet diese Publikation in der Deutschen Nationalbibliografie; detaillierte bibliografische Daten sind im Internet über <http://dnb.ddb.de> abrufbar. Open Access: The online version of this publication is published on www.peterlang.com and www.econstor.eu under the international Creative Commons License CC-BY 4.0. Learn more on how you can use and share this work: http://creativecommons. org/licenses/by/4.0.

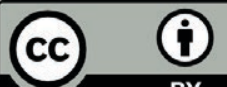

BY

This book is available Open Access thanks to the kind support of ZBW - Leibniz-Informationszentrum Wirtschaft.

Zugl.: Wien, Wirtschaftsuniv., Diss., 2004

Gefördert durch die Wirtschaftsuniversität Wien.

Für die Grafiken dieses Bandes standen teilweise nur unzureichende Vorlagen zur Verfügung.

Wir bitten um Verständnis dafür, daß wir sie aus dokumentarischen Gründen dennoch abgedruckt haben.

Gedruckt auf alterungsbeständigem, säurefreiem Papier.

ISSN 1613-3056

ISBN3-631-53881-2

ISBN 978-3-631-75411-5 (eBook)

(C) Peter Lang $\mathrm{GmbH}$

Europäischer Verlag der Wissenschaften

Frankfurt am Main 2005

Alle Rechte vorbehalten.

Das Werk einschließlich aller seiner Teile ist urheberrechtlich geschützt. Jede Verwertung außerhalb der engen Grenzen des Urheberrechtsgesetzes ist ohne Zustimmung des Verlages unzulässig und strafbar. Das gilt insbesondere für Vervielfältigungen, Übersetzungen, Mikroverfilmungen und die Einspeicherung und Verarbeitung in elektronischen Systemen.

Printed in Germany 124567 www.peterlang.de 
An dieser Stelle möchte ich mich herzlich bei all jenen bedanken, die mich während der Erstellung dieser Arbeit unterstützt haben. Besonderer Dank gilt meinen Doktorvätern Herrn Professor Dietmar Rössl, Wien und Herrn Professor Josef Mugler, Wien für die hervorragende Betreuung. Die zahlreichen anregenden Gespräche und Diskussionen haben ebenso ganz wesentlich zum Gelingen der Arbeit beigetragen wie die Ressourcen, die mir Herr Professor Mugler freundlicherweise an seinem Institut zur Verfügung gestellt hat.

Meiner Familie und meiner Freundin Cosima danke ich dafür, dass es mir in den letzten achtzehn Monaten ermöglicht wurde, mich ganz auf das Dissertationsprojekt zu konzentrieren. 

„Kein noch so drakonisches Gesetz, kein noch so zahlreiches Polizisten- und Beamtenheer, keine noch so scharfe allgegenwärtige Kontrolle kann nämlich einen solchen Zwang ausüben wie das Vertrauen. Das sicherste, ja das einzige

Mittel, die Menschen anständig zu machen, besteht darin, dass man sie für anständig hält."

(Egon Friedell 1983, 108) 



\section{Geleitwort}

Angesichts des Entwicklungsstandes der Kooperationsforschung, die nach Jahren der Theorieentwicklung nunmehr vor allem erhebliche Defizite in der empirischen Plausibilitätsprüfung ihrer Aussagen aufweist, greift Matthias Fink in seiner bemerkenswerten Arbeit ein zentrales betriebswirtschaftliches Thema auf.

Im Rahmen der theoretischen Modellierung stellt er die Frage nach der Möglichkeit und dem Erfolg der Koordinationsleistung maximenbasierten Vertrauens auf ein philosophisches und soziologisches Fundament. So gelingt es Herm Fink, die bearbeitete Fragestellung in einen größeren Zusammenhang zu stellen, womit von der Arbeit auch über die Grenzen der Betriebswirtschaft hinausgehende Implikationen auf die Organisation wirtschaftlicher Aktivitäten ausgehen.

Auch wenn die geleistete theoretische Arbeit einen wesentlichen Beitrag zur Integration der Kooperationsforschung zu leisten vermag, stellte die Messbarmachung des entwickelten Modells das weitaus riskantere, aber umso spannendere Unterfangen dar. Neben ausgeprägten analytischen Fähigkeiten und einer fundierten Methodenkenntnis bedarf es auch der nötigen Kreativität, um die mit dem bearbeiteten Untersuchungsgegenstand verbundene Operationalisierungsproblematik überwinden und das Modell einer empirischen Überprüfung zugänglich machen zu können. Mit dieser empirischen Studie und den bereits vorgelegten weiterführenden Forschungsarbeiten erschließt Matthias Fink zentrale, bisher offene Fragen für die Kooperationsforschung. Seine Ergebnisse und vor allem sein Forschungsdesign werden sowohl die weitere Theoriebildung als auch die empirischen Arbeiten im Forschungsfeld der zwischenbetrieblichen Kooperationen beeinflussen.

Die vom Verständnis um die gegenseitige Beeinflussung von theoretischer und empirischer Forschung getragene Arbeit verdient nicht zuletzt auf Grund der Präzision der analytischen Arbeit, ihrer Klarheit und Aussagekraft, besonders aber wegen ihrer potenziell katalytischen Wirkung auf die empirische Kooperationsforschung eine besondere Beachtung in der Fachwelt. 



\section{Inhaltsübersicht}

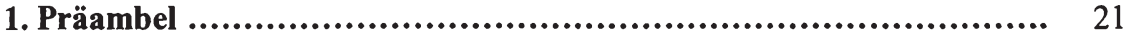

2. Wissenschaftstheoretische Positionierung ............................... 23

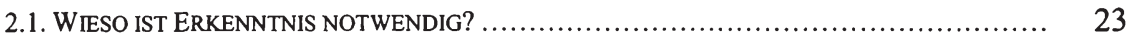

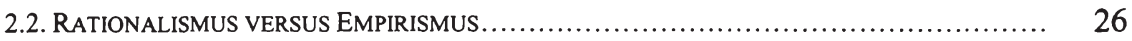

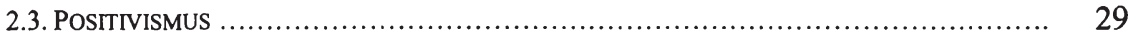

2.4. ARGUMENTATIONSLINIEN DER POSITIVISMUSKRITIK .................................. 30

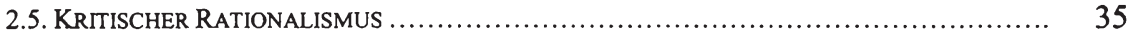

2.6. KRITIK DES KRITISCHEN RATIONALISMUS ........................................... 35

2.7. ALTERNATIVEN ZUM POSITIVISMUS ......................................................... 39

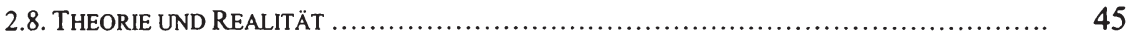

2.9. KONSEQUENZEN FÜR DIE VORLIEGENDE ARBEIT ....................................... 48

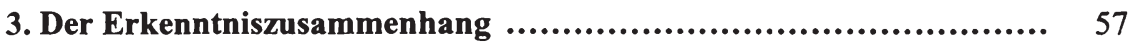

3.1. VON DER UNSICHERHEIT ZUM VERTRAUEN ......................................... 57

3.2. VOM VeRTRAUEN ZUR SELBSTVERPFLICHTUNG ..................................... 71

3.3. DAS MAXIMENBASIERTE VERTRAUENSVERHÄLTNIS ................................. 75

3.4. SELBSTVERPFLICHTUNG ALS MÖGLICHKEIT ZUR DURCHBRECHUNG DOPPELTER
KONTINGENZ

4. Formulierung der Forschungsfrage und Arbeitshypothese............. 83

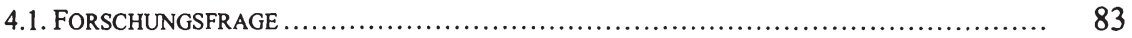

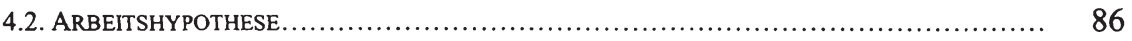

4.3. FORSCHUNGSDESIGN UND WEITERE ENTWICKLUNG DES TEXTES _....................... 87

5. Die empirische Erhebung ............................................ 89

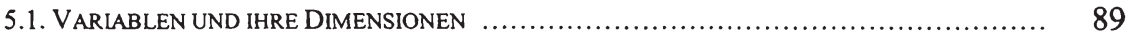

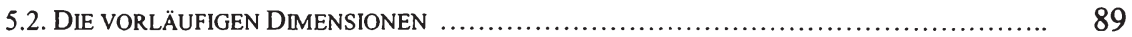

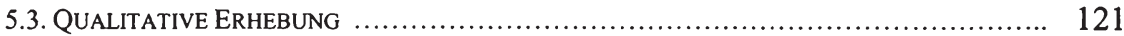

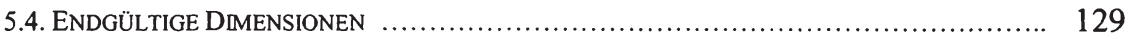

5.5. MODELLIERUNG DER INTERDEPENDENZEN ZWISCHEN DEN DIMENSIONEN DER LATENTEN VARIABLEN - ENTWICKLUNG EINES GESAMTMODELLS _.............................. 130

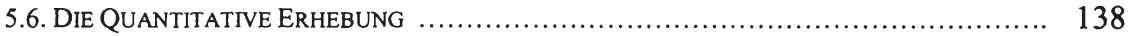

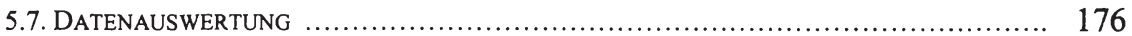

6. Zusammenfassende Thesen - Was wurde erreicht?................... 205

APPENDIX 1: ITEMNUMMER, LABEL (SPSS 10) UND ITEMFORMULIERUNG …............... 207 
APPENDIX 2: DATENMATRIX

APPENDIX 3: RELIABILITÄTSTEST

APPENDIX 4: KONSISTENZTEST - TRENNSCHÄRFEKOEFFIZIENTEN .

APPENDIX 5: FORMELN AUS SPSS 10 243

APPENDIX 6: KORRELATIONSMATRIZEN 245

LISTE DER ZITIERTEN LITERATUR 247 


\section{Inhaltsverzeichnis}

1. Präambel.............................................................. 21

2. Wissenschaftstheoretische Positionierung $. . . . \ldots \ldots \ldots \ldots \ldots \ldots \ldots \ldots \ldots \ldots . . . \ldots 23$

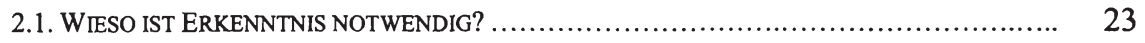

2.1.1. Entscheidung und Erkenntnis ...................................................... 23

2.1.2. Wissenschaft als sozial institutionalisierte Erkenntnis ................................ 25

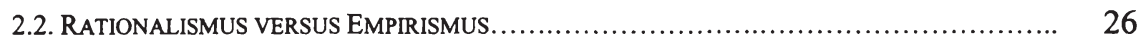

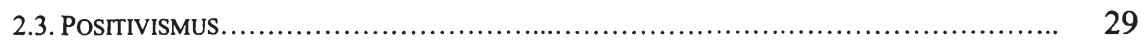

2.4. ARGUMENTATIONSLINIEN DER POSITIVISMUSKRITIK................................. 30

2.4.1. Die Geisteswissenschaften und die ,Zwei-Welten-Theorie“ ........................... 30

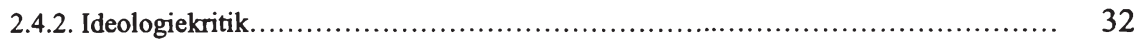

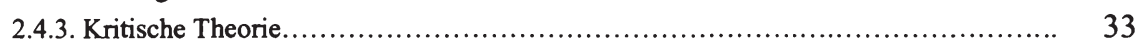

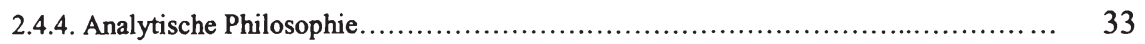

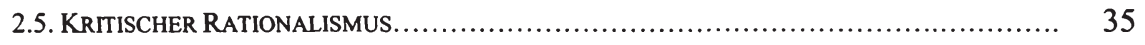

2.6. KRITIK DES KRITISCHEN RATIONALISMUS.................................................. 35

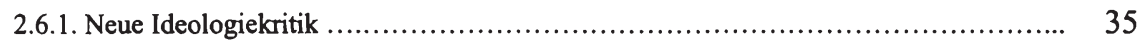

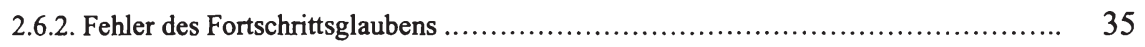

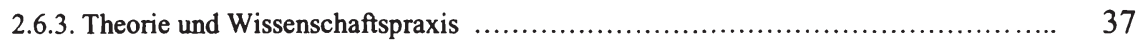

2.6.4. Befreiung vom Positivismus............................................................ 38

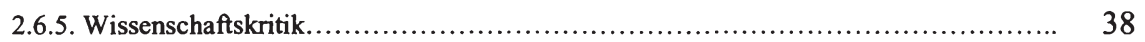

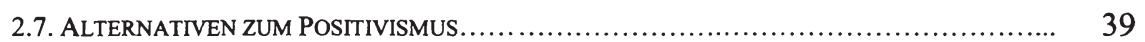

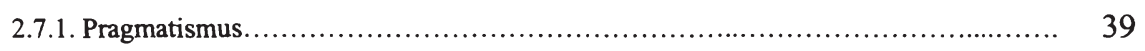

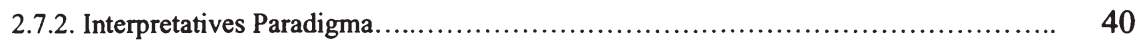

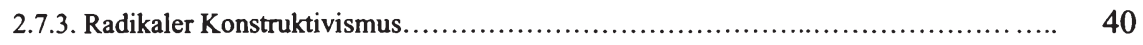

2.7.4. Evolutionäre Erkenntnistheorie ...................................................... 41

2.7.5. Genetische Erkenntnistheorie........................................................ 42

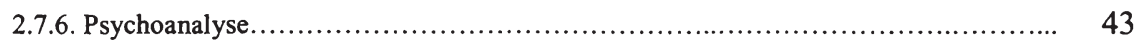

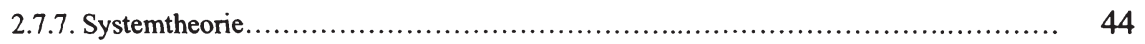

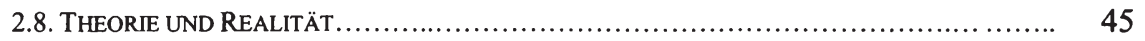

2.8.1. Realität - Logisch unterschiedliche Gegenstände.................................. 45

2.8.2. Von logisch unterschiedlichen Theorien zur Forderung nach Multiparadigmatik....... 46

2.8.3. Erkenntnistheorie als konnotative Theorie............................................ 47

2.8.4. Unabschließbarkeit der Erkenntnistheoriearbeit...................................... 48



2.9.1. Intersubjektive Transparenz und dokumentierte Entscheidungen...................... 49

2.9.2. Durch gutes Erklären Verstehen sichern.............................................. 50

2.9.3. Die Forschergemeinde als kritisches Korrektiv ......................................... 50

2.9.4. Ideen- und Methodenpluralismus................................................ 51

2.9.5. Pragmatisch nützliche Aussagen.......................................................... 51 


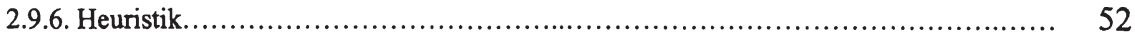

2.9.7. Verantwortlichkeit des Forschers für ,seine“ Wirklichkeitskonstruktion............... 53

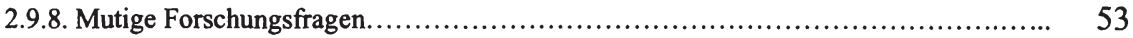

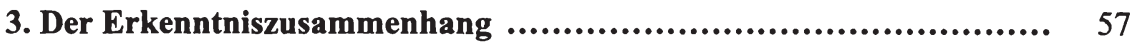

3.1. VON DER UNSICHERHEIT ZUM VERTRAUEN.............................................. 57

3.1.1. Unbrauchbare Annahmen........................................................ 57

3.1.2. Identifikation eines brauchbaren Vertrauensbegriffs................................... 59

3.1.2.1. Abgrenzung gegen den Begriff ,Zuversicht" “...................................... 59

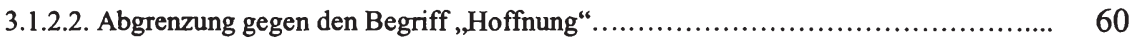

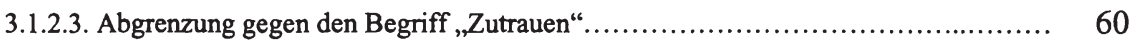

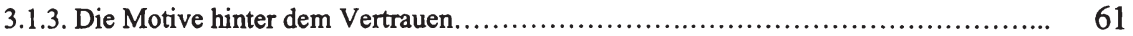

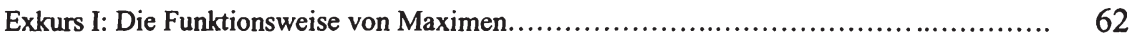

Exkurs II: Die Möglichkeit moralischer Handlungen im Markt - Warum das

Wirtschaftssubjekt kooperativ handeln ,soll" ...................................... 65

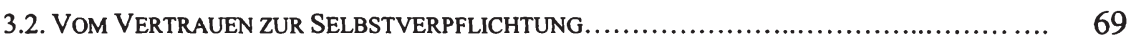

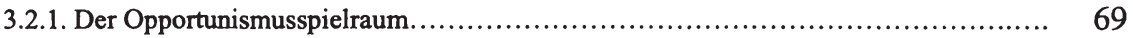

3.2.2. Begrenzung des Opportunismusspielraums als Komplexitätsreduktionsstrategie....... 70

3.2.3. Begrenzung der Opportunismusneigung als Komplexitätsabsorptionsstrategie.......... 73

3.3. DAS MAXIMENBASIERTE VERTRAUENSVERHäLTNIS ..................................... 75

3.4. SELBSTVERPFLICHTUNG ALS MÖGLICHKEIT ZUR DURCHBRECHUNG DOPPELTER

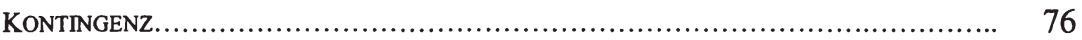

4. Formulierung der Forschungsfrage und Arbeitshypothese............. 83

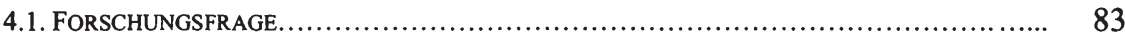

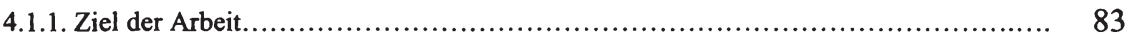

4.1.2. Warum die Existenz von Erfolgsfaktoren vermutet wird................................ 83

4.1.3. Selbstverpflichtung als Erfolgsfaktor in Synergiesystemen............................. 83

4.1.4. Selbstverpflichtung als Erfolgsfaktor in Synergiesystemen unter Unternehmen.......... 83

4.1.5. Selbstverpflichtung als Erfolgsfaktor bei Synergiesystemen unter KMU................ 85

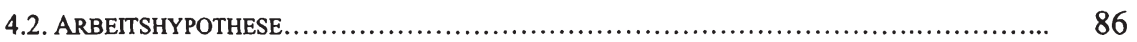

4.3. FORSCHUNGSDESIGN UND WEITERE ENTWICKLUUNG DES TEXTES........................ 87

5. Die empirische Erhebung............................................. 89

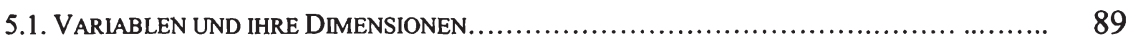

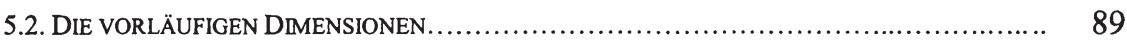

5.2.1. Kategorisierungsvariable „Synergiesystem“.......................................... 89

5.2.1.1. Drei Perspektiven des Möglichkeitsraums........................................... 92

5.2.1.2. Die Charakteristika des Synergiesystems........................................ 95

5.2.1.2.1. Charakteristika des Begriffs Kooperation.......................................... 95

5.2.1.2.2. Zusätzliche Charakteristika des Begriffs Synergiesystem............................ 97

5.2.2. Erklärende Variable „Selbstverpflichtung“.......................................... 98

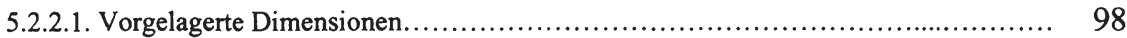


5.2.2.1.1. Die Wirkung der Erfahrung auf das menschliche Verhalten....................... 98

5.2.2.1.2. Vertrautheit, Reputation und perzepierte Geschichte......................... 101

5.2.2.1.3. Risikobereitschaft und Selbstvertrauen.................................... 103

5.2.2.2. Nachgelagerte Dimension: Unaufgeforderte Erhöhung der eigenen Gefahr........... 104

5.2.3. Abhängige Variable „Erfolg“ “.............................................. 105

5.2.3.1. Dimensionen der Beziehungsperspektive..................................... 107

5.2.3.1.1. Kommunikationsqualität................................................. 107

5.2.3.1.2. Stabilität und Belastbarkeit - Verlässlichkeit............................... 107

5.2.3.1.3. Sympathie und persönliche Beziehung................................... 107

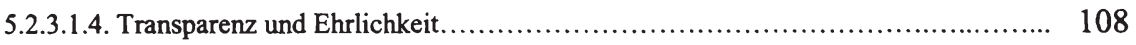

5.2.3.1.5. Entscheidungsfindung................................................ 108

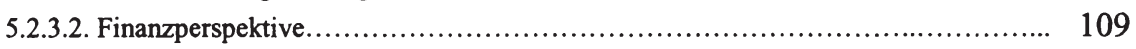

5.2.3.2.1. Umsatzbezogene Kennzahlen............................................ 110

5.2 .3 .2 .2 . Rentabilitätskennzahlen................................................ 111

5.2.3.3. Binnenperspektive..................................................... 112

5.2.3.3.1. Mitarbeiterfluktuation................................................ 112

5.2.3.3.2. Verlässlichkeit der Prognosen........................................... 114

5.2.3.4. Außenperspektive......................................................... 115

5.2.3.4.1. Kundenzufriedenheit und Stammkundenanteil.............................. 115

5.2.3.4.2. Marktanteilsentwicklung................................................. 116

5.2.3.4.3. Anteil der Stammlieferanten.............................................. 118

5.2.3.4.4. Angebots-Zuschlagsrate.............................................. 119

5.2.4. Zusammenfassung: Die vorläufigen Dimensionen der Variablen.................... 119

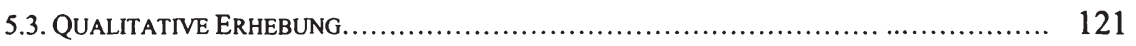

5.3.1. Verworfene Dimensionen..................................................... 123

5.3.1.1. Verworfene Dimension „Umsatzrentabilität“

5.3.2. Adaptierte Dimensionen..................................................... 124

5.3.2.1. Adaptierte Dimension ,langfristige Perspektive“.............................. 124

5.3.2.2. Adaptierte Dimension ,Flexibilität““..................................... 124

5.3.2.3. Adaptierte Dimension ,Risikobereitschaft“.................................. 125

5.3.2.4. Adaptierte Dimension „Cash-Flow-Umsatzrate“ ............................... 125

5.3.2.5. Adaptierte Dimension ,Angebotszuschlagsrate“................................ 126

5.3.2.6. Adaptierte Dimension ,Anteil der Investitionen am Umsatz“.................... 126

5.3.2.7. Adaptierte Dimension „Kundenzufriedenheit und Stammkundenanteil“............. 126

5.3.3. Zusätzlich in die Erhebung integrierte Dimensionen.............................. 126

5.3.3.1. Zusätzliche Dimension „Mitarbeiterqualifikation“.............................. 127

5.3.3.2. Zusätzliche Dimension „Entwicklung der Beziehungsintensität“................... 127

5.4. ENDGÜltige DiMENSIONEN...................................................... 127

5.5. MODELLIERUNG DER INTERDEPENDENZEN ZWISCHEN DEN DIMENSIONEN DER LATENTEN VARIABLEN - ENTWICKLUNG EINES GESAMTMODELL.............................. 130 
5.5.1. Entwicklung der Messmodelle ............................................... 130

5.5.1.1. Messmodell der Variablen Synergiesystem“................................... 130

5.5.1.2. Messmodell der Variablen ,Selbstverpflichtung“............................. 131

5.5.1.3. Messmodell der Variablen ,Erfolg“ “....................................... 132

5.5.2. Entwicklung des Strukturmodells.......................................... 135

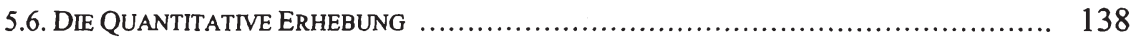

5.6.1. Untersuchungseinheiten und Befragte ........................................ 138

5.6.2. Entwicklung des Erhebungsinstrumentes................................... 139

5.6.3. Grundsätze der Fragebogengestaltung ....................................... 139



5.6.3.2. Bestimmtheit ........................................................ 141

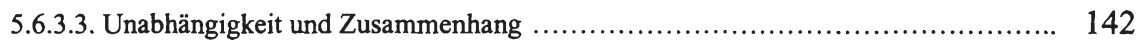

5.6.3.4. Bescheidenheit und Notwendigkeit ....................................... 144

5.6.3.5. Klarheit und Übersichtlichkeit ............................................ 145

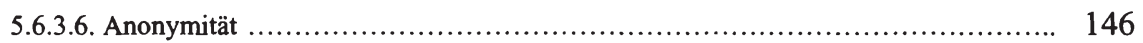

5.6.4. Statements als Items .................................................... 146

5.6.5. Antwortkategorien, Messskala und Skalenniveau ............................... 147

5.6.6. Entwicklung der Einzelitems ................................................. 147

5.6.6.1. Operationalisierung der Dimensionen der latenten Variablen „Synergiesystem“..... 148

5.6.6.2. Operationalisierung der Dimensionen der latenten Variablen „Selbstverpflichtung“.. 153

5.6.6.3. Operationalisierung der Dimensionen der latenten Variablen „Erfolg aus der

Beziehungsperspektive“................................................ 157

5.6.6.4. Operationalisierung der Dimensionen der latenten Variablen „Erfolg aus der

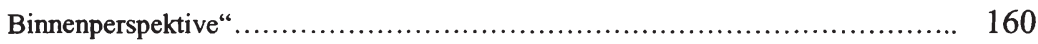

5.6.6.5. Operationalisierung der Dimensionen der latenten Variablen „Erfolg aus der

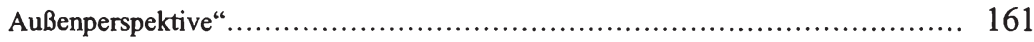

5.6.6.6. Operationalisierung der Dimensionen der latenten Variablen „Erfolg aus der

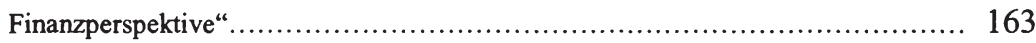

5.6.6.7. Zusätzliches Item ,Kooperationsdauer“...................................... 164

5.6.7. Die Verbindungstabelle ....................................................... 164

5.6.8. Überlegungen zu Fragensequenzierung und Fragebogenstrukturierung................ 168

5.6.9. Untersuchungszeitraum, Grundgesamtheit, Stichprobe und Rücklaufquote............ 170

5.7. DatenausWERTUNG ........................................................ 176

5.7.1. A posteriori Prüfung der Güte des Erhebungsinstruments ......................... 176

Exkurs III: Ursachen, Wirkungen und der Pfeil .................................... 176

5.7.1.1. Prüfung der Güte der Fragebogenitems mittels Trennschärfekoeffizient ............ 177

5.7.1.1.1. Prüfung der Güte der Items zur Erhebung der Ausprägungen der latenten Variablen ,Synergiesystem“....................................... 177

5.7.1.1.2. Prüfung der Güte der Items zur Erhebung der Ausprägungen der latenten Variablen „Selbstverpflichtung“... 
5.7.1.1.3. Prüfung der Güte der Items zur Erhebung der Ausprägungen der latenten Variablen „Erfolg aus der Beziehungsperspektive“

5.7.1.1.4. Prüfung der Güte der Items zur Erhebung der Ausprägungen der latenten Variablen ,Erfolg aus der Binnenperspektive“....

5.7.1.1.5. Prüfung der Güte der Items zur Erhebung der Ausprägungen der latenten Variablen „Erfolg aus der Außenperspektive“.

5.7.1.1.6. Prüfung der Güte der Items zur Erhebung der Ausprägungen der latenten Variablen „Erfolg aus der Finanzperspektive“

5.7.1.2. Test auf Reliabilität mittels der Split-half Methode

5.7.2. Überprüfung der Gültigkeit des theoretischen Modells

5.7.2.1. Überprüfung der Gültigkeit der Messmodelle

5.7.2.1.1. Überprüfung des Messmodells der latenten Variablen „Synergiesystem“.

5.7.2.1.2. Überprüfung des Messmodells der latenten Variablen „Selbstverpflichtung“

5.7.2.1.3. Überprüfung des Messmodells der vier Perspektiven der latenten Variablen „Erfolg“"

5.7.2.1.4. Überprüfung der Struktur der verwendeten Balanced Scorecard

5.7.2.2. Überprüfung der Gültigkeit des Strukturmodells

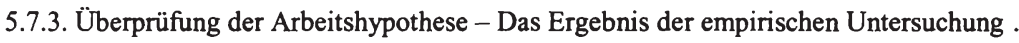
200

6. Zusammenfassende Thesen - Was wurde erreicht? 205

APPENDIX 1: ITEMNUMMER, LABEL (SPSS 10) UND ITEMFORMULIERUNG

APPENDIX 2: DATENMATRI

APPENDIX 3: RELIABILITÄTSTEST

APPENDIX 4: KONSISTENZTEST - TRENNSCHÄRFEKOEFFIZIENTEN

APPENDIX 5: FORMELN AUS SPSS 10

APPENDIX 6: KORRELATIONSMATRLEN 



\section{Abbildungsverzeichnis}

Abb. 1: Empirismus, Rationalismus, Kant und Hegel .................... 28

Abb. 2: Programme der analytischen Philosophie ........................ 34

Abb. 3: Gang der Argumentation..................................... 71

Abb. 4: Entstehungsprozess eines maximenbasierten

Vertrauensverhältnisses ...................................... 76

Abb. 5: Maximenbasiertes Vertrauen ermöglicht

Transaktionsbeziehungen .................................. $\quad 79$

Abb. 6: Transaktionsbeziehungen und ihre Basen ...................... 80

Abb. 7: Argumentationsweg und Forschungsfrage .................... 84

Abb. 8: Entwicklung der Kapitel im Text und der Arbeitsschritte des

Projekts ...................................................... 87

Abb. 9: Operationalisierungsschema ................................. 90

Abb. 10: Isolierung des Begriffs, „Synergiesystem“ im Möglichkeitsraum.. 94

Abb. 11: Zusammenfassende Darstellung der Dimensionen eines

Synergiesystems ............................................... 98

Abb. 12: Einfluss der Erfahrung auf das Verhalten ..................... 100

Abb. 13: Vergleich und Abgrenzung der Begriffe Reputation, Vertrautheit und Erfahrung

Abb. 14: Zusammenfassende Darstellung der Dimensionen der

erklärenden Variablen „Selbstverpflichtung“................... 104

Abb. 15: Struktur der Balanced Scorecard zur Erhebung der abhängigen

Variablen Erfolg ......................................... 106

Abb. 16: Die vorläufigen Dimensionen der Variablen - ein Überblick ..... 120

Abb. 17: Zuordnung der Kompetenzfelder zu den Gesprächspartnern ...... 122

Abb. 18: Auflistung der möglichen Szenarien in der Periode t0 bis t1 ..... 128

Abb. 19: Die endgültigen Dimensionen der Variablen - ein Überblick ..... 129

Abb. 20: Messmodell der Variablen „Synergiesystem“.................... 131

Abb. 21: Messmodell der Variablen „Selbstverpflichtung“................ 132

Abb. 22: Balanced Scorecard als formale Struktur der Erfolgsmessung .... 133

Abb. 23: Messmodell der Variablen „Erfolg“............................ 134

Abb. 24: Das Strukturmodell .............................................. 136

Abb. 25: Gesamtmodell .............................................. 137

Abb. 26: Trade-off zwischen Vollständigkeit und Rücklaufquote .......... 144

Abb. 27: Verbindungstabelle ........................................... 165

Abb. 28: Filterwirkung und Struktur des ersten Items .................... 169

Abb. 29: Der Fragebogen .......................................... 171

Abb. 30: Trennschärfekoeffizienten der Itembatterie „Synergiesystem“.... 177

Abb. 31: Güte der Itembatterie „Synergiesystem“........................ 179

Abb. 32: Trennschärfekoeffizienten der Itembatterie „Selbstverpflichtung“ 180

Abb. 33: Güte der Itembatterie „Selbstverpflichtung““................... 181

Abb. 34: Trennschärfekoeffizienten der Itembatterie „Erfolg aus der

Beziehungsperspektive“....................................... 182

Abb. 35: Güte der Itembatterie „Erfolg aus der Beziehungsperspektive“... 183 
Abb. 36: Trennschärfekoeffizienten der Itembatterie „Erfolg aus der Binnenperspektive“

Abb. 37: Güte der Itembatterie „Erfolg aus der Binnenperspektive“...

Abb. 38: Trennschärfekoeffizienten der Itembatterie „Erfolg aus der

Abb. 39: Güße der Itembatter

,Erfolg aus der Außenperspektive“

Abb. 40: Trennschärfekoeffizienten der Itembatterie „Erfolg aus der Finanzperspektive"

Abb. 41: Güte der Itembatterie „Erfolg aus der Finanzperspektive“........ 188

Abb. 42: Ergebnisse des Reliabilitätstests .............................. 189

Abb. 43: Die Komplexität des Forschungsgegenstandes .................. 190

Abb. 44: Überprüfung des Messmodells der Variablen „Synergiesystem”.. 192

Abb. 45: Überprüfung des Messmodells der Variablen „Selbstverpflichtung”....

Abb. 46: Adaptiertes Modell der Variablen „Selbstverpflichtung“.......... 195

Abb. 47: Überprüfung des Messmodells der Variablen „Erfolg“............ 197

Abb. 48: Überprüfung der Struktur der Balanced Scorecard................ 198

Abb. 49: Überprüfung des Strukturmodells ............................ 200

Abb. 50: Die Arbeitshypothese ................................... 201

Abb. 51: Hypothesentest als Überprüfung der Gültigkeit des Argumentationsweges...................................... 202

Abb. 52: Test der Teilhypothese 2 - Korrelationskoeffizienten ............ 203 


\section{Präambel}

Egon Friedell betont in der zitierten Textpassage die außerordentlich starke normative Kraft, die von intrinsisch motiviertem (maximenbasiertem) Vertrauen ausgeht. Durch seine verhaltensnormierende Wirkung stellt Vertrauen einen wirkungsvollen Mechanismus zur Koordination sozialer Beziehungen dar. Es stellt sich die Frage, ob dieser allgemeine Koordinationsmechanismus auch in Bereichen der Gesellschaft erfolgreich seine Wirkung entfalten kann, in denen bisher vor allem der Marktmechanismus und hierarchische Strukturen für die Abstimmung der Transaktionsbeziehungen verantwortlich gemacht wurden. Diese zwei (Markt und Hierarchie), in der klassischen Betriebswirtschaft behandelten Koordinationsmechanismen reichen für die Erklärung vieler im realen Wirtschaftsleben stattfindender Transaktionsbeziehungen jedoch offensichtlich nicht aus: Nach Rößl' sind davon jene Transaktionsbeziehungen betroffen, bei denen

„,weder der Markt noch die hierarchische Organisation [...] geeignete Koordinationsmechanismen [darstellen], wenn eine auf langfristige Vorteile abzielende Transaktion hochgradig unsicher und komplex ist, sodass ein hierarchisches Festlegen des Verhaltens der Unsicherheit nicht angepasst wäre. D.h., einerseits steht die Langfristigkeit einer verlässlichen Koordination durch den Marktmechanismus entgegen, umgekehrt erlaubt die mangelnde Programmierbarkeit der Beziehung keine hierarchische Koordination. “2

Obwohl das Markt- bzw. Organisationsversagen zu einem Unterbleiben der betroffenen Transaktionen führen müsste, finden diese in der Realität jedoch sehr wohl statt. Diese Arbeit unternimmt den Versuch, die Frage nach der Möglichkeit, der Notwendigkeit und dem Erfolg der Entfaltung der Koordinationsleistung von maximenbasiertem Vertrauen im betriebs-wirtschaftlichen Kontext der langfristigen, hochgradig komplexen und unsicheren zwischenbetrieblichen Kooperationsbeziehung (Synergiesysteme ${ }^{3}$ ) stringent $\mathrm{zu}$ argumentieren, sowie diese Argumentation auf der Basis einer Befragung von Geschäftsführern kleiner und mittlerer Unternehmen fundiert empirisch zu überprüfen.

Die Frage nach der Möglichkeit des Einsatzes von Vertrauen zur Koordination von Transaktionsbeziehungen verweist auf die Überprüfung von zwei Voraussetzungen. Erstens ist zu beleuchten, ob die Wirtschaftssubjekte angesichts der im Wirtschaftssystem herrschenden Restriktionen überhaupt vertrauen können. Zweitens ist zu begründen, warum sie als Vertrauende bzw. als Vertrauensempfänger die vom Vertrauen ausgehende Einschränkung ihres Handlungsspiel-

\footnotetext{
1 Rößl (1994a) sowie Rößl (1994b) und Osterloh/Weibel(2000)

2 Röß1 1994b, 11

3 Zum Begriff Synergiesysteme siehe Abschnitt 5.2.1.
} 
raums zulassen sollen. Erst die Rechtfertigung der normativen Kraft instrumentellen Vertrauens eröffnet den Argumentationsweg für dessen Notwendigkeit als Schlüssel zur Lösung sozialer Dilemmata von der Art des Gefangenendilemmas.

Hochgradig komplexe und unsichere Transaktionsbeziehungen, die auf eine langfristige Zielerreichung angelegt sind (Synergiesysteme), stellen solche sozialen Dilemmata dar. Es ist zu zeigen, wie die, auf maximenbasiertem Vertrauen beruhende Selbstverpflichtung der Akteure, die in solchen Situationen bestehende wechselseitige Verhaltensunsicherheit („Doppelte Kontingenz“) durchbrechen kann und so die Etablierung und den Bestand von Synergiesystemen ermöglicht. Gelingt es des weiteren, Selbstverpflichtung als vergleichsweise ressourcenschonende Alternative zur Überwindung von Komplexität und Unsicherheit in Austauschbeziehungen herauszustellen, so ist eine gültige Argumentation für Selbstverpflichtung als ein Erfolgsfaktor bei Synergiesystemen gefunden.

In einem weiteren Schritt ist die formulierte Argumentation mit der Empirie zu konfrontieren. Dazu werden die relevanten abstrakten Phänomene

- Selbstverpflichtung,

- Synergiesystem und

- Unternehmenserfolg (Messmodelle)

sowie die in der Argumentation behaupteten Wirkungszusammenhänge (Strukturmodell) in einem mehrstufigen Operationalisierungsprozess in ein empirisch überprüfbares Gesamtmodell übersetzt. Sodann ist ein Fragebogen zu entwerfen, der im Rahmen einer großzahligen Erhebung die tatsächlichen Ausprägungen der relevanten Phänomene erfassen kann. Inwieweit die im Gesamtmodell formulierten Kausalstrukturen als Muster in den erhobenen Daten identifiziert werden können, entscheidet über die Gültigkeit des Modells und damit über das Schicksal der theoretisch geführten Argumentation.

Bevor das oben entworfene Unternehmen in Angriff genommen wird, ist jedoch noch ein wissenschaftstheoretisches Fundament zu formulieren, das den Geltungsanspruch der in der Arbeit getroffenen Aussagen rechtfertigt und die Reichweite dieses Anspruchs absteckt. 


\section{Wissenschaftstheoretische Positionierung}

Das Ziel jeder ernsthaften wissenschaftlichen Tätigkeit ist die Generierung von Wissen. Daher sollte eine wissenschaftliche Arbeit die Zielsetzung haben etwas Neues herauszufinden und an die Öffentlichkeit zu transportieren. Das bedeutet, dass jede wissenschaftliche Arbeit auf Erkenntnis abzielt. Damit ist sie automatisch mit den Problemen der Erkenntnis konfrontiert. Es ist zwar praktisch möglich diese grundsätzlichen Fragen auszuklammern und sich direkt in die Bearbeitung der jeweiligen Forschungsfrage zu stürzen, es ist aber äußerst fraglich, ob eine Erklärung ohne Begründung ${ }^{4}$ befriedigend sein kann. Auch diese Arbeit wird das Erkenntnisproblem nicht lösen können, aber dessen (wenn auch knappe) Diskussion und die daraus gezogenen Schlüsse können eine hinreichend stabile Basis für wissenschaftliche Aussagen darstellen, auch wenn diese, und das möge sich der Leser bei der Lektüre vergegenwärtigen, von definitiven Begründungen weit entfernt sind.

Der Schaffung dieser hinreichend stabilen Basis widmet sich der erste Teil der Arbeit. Zuerst wird in einem ersten Schritt danach gefragt, warum Erkenntnis für den Menschen notwendig ist, um dann in einem zweiten Schritt die Frage nach der Möglichkeit von Erkenntnis aufzuwerfen. Um dem Anspruch auf Klarheit auch bezüglich der inhaltlichen Entwicklung des Textes gerecht zu werden, wird die Diskussion der Antworten auf die Frage nach der Möglichkeit von Erkenntnis chronologisch dargestellt. Im Anschluss werden zuerst die Konsequenzen dieser Diskussion für die vorliegende Arbeit besprochen, um dann Grundsätze zu formulieren, die das wissenschaftstheoretische Fundament dieser Arbeit bilden.

\subsection{Wieso ist Erkenntnis notwendig?}

\subsubsection{ENTSCHEIDUNG UND ERKENNTNIS}

Unbelebte Dinge unterliegen den Natur"gesetzen". Sie haben keine Wahl, ob und inwiefern sie diesen Folge leisten wollen, denn sie können nicht wollen. Sie brauchen aber auch nicht wollen können, da sie nicht wählen müssen, um zu überleben. Lebewesen sind im Unterschied dazu jedoch ständig mit der Notwendigkeit konfrontiert, ihr Leben zu erhalten. Lebewesen sind a priori Programme gegeben,

\footnotetext{
4 Erklärungen stellen immer Aussagen über Zusammenhänge dar. Um Zusammenhänge erklären zu können, muss man sie zuerst erkannt haben. Erkenntnis muss ihre Bedingungen offen legen, um auf einem stabilen Fundament zu stehen. Diese Offenlegung der Bedingungen der Möglichkeit von Erkenntnis kann nur eine Metha-Theorie (Erkenntnistheorie/Wissenschaftstheorie) leisten. Sie fungiert dann als Begründung der Erkenntnis.
} 
die bei gewissen Reizen bzw. Reizkonstellationen auf das rezeptorische System ein ganz bestimmtes Verhalten des Lebewesens notwendigerweise auslösen. So ist das Überleben eines solchen Lebewesens in stabilen Umwelten potenziell gesichert. Mit der unmittelbaren Wirkung der Reize auf das Verhalten ist sowohl eine große Sicherheit des Vorganges, aber gleichzeitig auch ein hohes Maß an Inflexibilität verbunden. Veränderungen der Umwelt stellen Lebewesen, die durch Programmierung ihr Überleben zu sichern suchen, vor unlösbare Probleme: Lernen ist nicht dem einzelnen Individuum möglich, sondern nur der gesamten Population und wird von Generation zu Generation im Genom weitergegeben. ${ }^{5}$

Im Laufe der Evolution entwickelte sich eine andere Methode im Umgang der Lebewesen mit dem Problem der Überlebenssicherung. Die Programmierung nahm ab. So entstand für die Lebewesen ein Spielraum, der ihnen ermöglichte, sich auf geänderte Umweltbedingungen einzustellen. Gleichzeitig mit der neu gewonnenen Flexibilität mussten die Lebewesen einer völlig neuen Situation gerecht werden: Sie mussten handeln. Handlungen als praktischer Ausdruck des Willens basieren auf Entscheidungen, als deren Grundlage eine Kenntnis von der Welt Voraussetzung ist. Verkürzt ausgedrückt heißt das: Eine Reduzierung der Programmierung zwingt die Lebewesen zu handeln; Handlungen zwingen die Lebewesen Entscheidungen zu treffen; Entscheidungen zwingen die Lebewesen dazu sich Wissen über die Welt anzueignen. Anders formuliert bedeutet das: Entscheiden bedingt Erkenntnis. ${ }^{6}$

Es kann zwischen zwei Modi unterschieden werden, mit deren Hilfe der Mensch sein Überleben in der Welt sicherstellt. Auf der Ebene des Alltagsbewusstseins werden Routinen abgespielt, die dem Menschen eine kognitive Auseinandersetzung mit konkreten Situationen ersparen, indem bei Handlungen auf Erfahrungen zurückgegriffen wird. Dieser Modus ist zwar in Hinblick auf die knappen kognitiven Ressourcen sparsam, führt aber nur so lange zum Ziel, solange sich die Annahme erfüllt, dass die Vergangenheit in die Zukunft fort-geschrieben werden kann, also solange die Erfahrungen auf die konkrete, aktuelle Situation anwendbar sind. Ist das nicht der Fall, so steht der nach einer Routine handelnde Mensch vor einem Problem. Wäre ihm nur die Ebene des Alltagsbewusstseins zugänglich, so würde das Auftauchen eines Problems unweigerlich zum Scheitern führen. Glücklicherweise steht dem Menschen jedoch ein zweiter Modus zur

5 vgl. Schülein 2002, $10 \mathrm{ff}$

6 vgl. Schülein $2002,10 \mathrm{ff}$ 
Generierung von Handlungen zur Verfügung: die bewusste Entscheidung auf der Grundlage von Wissen.

Durch Institutionalisierung der Reflexion befreit die Gesellschaft den Akteur von permanentem Handlungsdruck und schafft so Freiräume, die es ihm ermöglichen, seine volle kognitive Kapazität auf Erkenntnis zu richten. Mit dem geschaffenen Freiraum steigt aber auch der Anspruch, der für die Reichweite der Gültigkeit der gewonnenen Erkenntnis erhoben wird. Sozial institutionalisierte Erkenntnis erhebt den Anspruch der Objektivität. Es handelt sich nicht mehr lediglich um Meinungen, die sich dem Anspruch der Legitimation entziehen, sondern um Theorien, die sich zu rechtfertigen haben, um dem Anspruch der Objektivität gerecht zu werden. Die soziale Institutionalisierung der Reflexion kann somit als Produktions-verfahren für objektive Erkenntnis unter der strengen Nebenbedingung der Effizienz gesehen werden. Das Produktionssystem wird Wissenschaft genannt. ${ }^{7}$

\subsubsection{WISSENSCHAFT ALS SOZIAL INSTITUTIONALISIERTE ERKENNTNIS}

Der Weg von der Behauptung zur Theorie führt über die Hürde der Legitimation des Anspruchs der objektiven Erkenntnis. Dies bedingt einerseits methodische Kontrolle und andererseits theoretische Fundierung. Wie dies geschieht, hängt von der unterstellten Theorie über die Theorien (Metha-Theorie) ab. Solche Metha-Theorien versuchen die Möglichkeit der Erkenntnis zu klären. Sie stellen eine Art „Dachorganisation“ für Theorienbildung dar, unter deren Schutzmantel Theorien, die den Ansprüchen der jeweiligen herangezogenen Metha-Theorie genügen, Objektivität beanspruchen können. Diese Verfahrensweise ist jedoch problematisch, da es sich bei Metha-Theorien ebenfalls um Theorien handelt, deren Anspruch auf Objektivität gerechtfertigt werden muss. So gerät man in einen infiniten Regress, dem sich die Wissenschaft, vor allem aber die Sozialwissenschaft, durch einen Regressstopp zu entziehen sucht. Leider können auch die in dieser Arbeit getroffenen Aussagen auf keine Letztbegründung zurückgreifen, auch ihrer wissenschaftstheoretischen Fundierung widerfährt notwendiger Weise ein Regressstop, dieser erfolgt jedoch bewusst und so spät wie möglich. Die Ursachen dieser unbefriedigenden Situation und die Diskussion ihrer Konsequenzen für die vorliegende Arbeit werden im Folgenden knapp dargestellt. Ausgangspunkt ist eine kurze Gegenüberstellung der erkenntnistheoretischen Grundhaltungen Rationalismus und Empirismus sowie eine knappe Besprechung deren wichtigster Vertreter.

7 vgl. Schülein $2002,22 \mathrm{ff}$ 


\subsection{Rationalismus versus Empirismus}

Rationalismus bezeichnet die erkenntnistheoretische Grundhaltung wonach die Welt durch die Idee erfasst und durch die Zuordnung von Begriffen zu Objekten geordnet werden kann. Die Idee erfasst das Objekt durch den Begriff so, wie es ist. ${ }^{8}$ Im Gegensatz dazu postuliert der Empirismus, dass es durch bestimmte Methoden (empirische Erfahrung und Systematisierung) zur Identität des realen mit dem wirklichen Objekt ${ }^{9}$ kommt $^{10}$ und dass so Erkenntnis möglich wird.

Als Vertreter der Empiristen seien die Grundzüge der Erkenntnisphilosophie von Francis Bacon dargestellt. Für ihn hat die oberste Wissenschaft die Aufgabe, die anderen Disziplinen zu kritisieren. Nur wenn wir uns von unseren Vorurteilen (Trugbildern) ${ }^{11}$ befreien, ist wahre Erkenntnis möglich. Laut Bacon ist ein wirkliches Abbild der Welt dem Menschen nur über ein Zusammenspiel von empirischer Erfahrung und einer ordnenden Tätigkeit des Geistes zugänglich.

Für den Rationalisten Descartes kann hingegen alles (die Welt) ein Irrtum sein und in Frage gestellt werden, außer dem Ich, das in Frage stellt (res cogitans) ${ }^{12}$. Dies ist daher der einzige definitive Ausgangspunkt für Erkenntnis. Es kommt so zur Trennung zwischen res cogitans (Subjekt) und der Welt (Objekt). Mit Hilfe der Universalmathematik kann die Welt in Ideen ${ }^{13}$ gefasst werden. Die Möglich-

$8 \mathrm{vgl}$. Müller/Halder 1973, $223 \mathrm{f}$.

9 ,Die richtige Erkenntnis ist erreicht, wenn das im Geiste vorhandene Bild mit der Wirklichkeit übereinstimmt (adequatio rei et intellectus)“. (Thomas von Aquin, zitiert in Störig 1992, 258)

10 Genauer dazu (Feyerabend 2002, 23ff) und (Schülein 2002, 67f)

11 Die vier Gruppen von Trugbildern sind: Trugbilder des Stammes: Der Mensch kann die Welt nur mit Hilfe seines Verstandes und seiner Sinne erfassen, sie geben aber ein verfälschtes Bild der Wirklichkeit. Trugbilder der Höhle: Jeder Mensch hat individuell Vorurteile je nach Veranlagung, Erziehung, Gewohnheit und Neigung. Trugbilder des Marktes: Im Bezug auf die Probleme der Sprache nimmt Bacon eine nominalistische Position ein. Die bloßen Namen sind mit der Logik nicht verbunden, sie entstehen im sozialen Umgang der Menschen (auf dem Markt). Trugbilder des Theaters: Es besteht die Gefahr, dass Theorien durch ihre Verschulung an Reflexion verlieren. So werden Intümer in den philosophischen Schulen tradiert und konserviert. Diese Schulen sind dann nur mehr Institutionen des Glaubens. (vgl. Bacon 1990, 164)

12 ,Ich habe mich überredet, dass es schlechterdings nichts in der Welt gibt: keinen Himmel, keine Erde keine Geister, keine Körper, also doch wohl mich selbst nicht? Keineswegs: ich war sicherlich, wenn ich mich dazu überredet habe. [...] Ich bin, ich existiere, soviel ist gewiss." (Descartes 1960, 100f)

13 „Ich [würde], was das Wachs ist nicht richtig beurteilen, wenn ich nicht der Meinung wäre, dass [...] es mehr Verschiedenheiten zulässt, als ich jemals in der Einbildung umfasst habe. Es bleibt mir nichts anderes übrig als zuzugeben, dass ich, was Wachs ist, gar nicht die Einbildung habe, sondern nur im Denken erfassen kann." (Descartes 1960, 104) 
keit von Ideen begründet Descartes durch die Existenz Gottes ${ }^{14}$. Auch Spinoza sieht in Gott die Letztbegründung. Der Mensch ist Teil der Natur (Naturalismus) und die Natur ist Gott (Monismus). Erkenntnis ist auf das Verständnis und auf die Folgeleistung der inneren Logik (der Notwendigkeit) des Geschehens gerichtet. Ist diese Logik verstanden, ist auch die Frage „Was ist Gott" beantwortet. ${ }^{15}$ Für Leibnitz besteht die Welt aus einer einzigen Substanz, die in zwei Arten von Monaden geteilt ist. Einerseits die nackten Monaden, die ein unbewusstes Verständnis der Welt haben und andererseits die beseelten Monaden (Menschen), die zwar zu bewusster, aber nur beschränkter Wahrnehmung fähig sind. Einzig Gott als Urmonade hat ein vollständiges Bild des Universums. Kurz: Wir und alles, was ist, ist Teil eines Ganzen. ${ }^{16}$

Kant überwindet den Widerspruch zwischen Empirismus und Rationalismus, indem er die Grundideen beider Denkrichtungen in seine Transzendentalphilosophie integriert. Er geht davon aus, dass sowohl Erfahrung als auch Denken notwendig sind und aufeinander verweisen. Er argumentiert, dass dem menschlichen Bewusstsein bestimmte Denkformen (Ordnung der Dinge in Raum und Zeit/die Kategorien) vorgegeben sind und erst Erfahrung ermöglichen. ${ }^{17}$ Erkennen ist eine aktive, produktive Leistung des Erkenntnissubjekts. Bei seiner Objektivierung richtet sich das Objekt nach der Theorie, da nur so eine Begrifflichkeit a priori möglich ist. Erst wenn wir von etwas einen Begriff haben, können wir beginnen, es wahrzunehmen. Kant stellt zwar fest, dass Erfahrung und Denken sich wechselseitig bedingen, betont aber die dominante Rolle des Verstandes. Die Konstruktionen des Verstandes können zwar an der Wirklichkeit überprüft werden, aber nur der Verstand kann Erkenntnis ermöglichen. Wir schaffen uns ein Abbild der Objekte. Die Dinge an sich sind der Erkenntnis nicht zugänglich. Das bedeutet, dass der reflektierte Gegenstand von dem realen Gegenstand systematisch verschieden ist (Konstruktivismus). ${ }^{18}$

14 „Unter dem Namen Gottes verstehe ich eine Substanz, die unendlich, unabhängig, von höchster Einheit und Macht ist, und von der ich selbst geschaffen worden bin, ebenso wie alles andere Existierende, fasst es nämlich existiert." (Descartes 1960, 113)

15 vgl. Störig 1992, 325f

16 vgl. Störig 1992, 337f

17 vgl. Kant 1988,201

$18 \mathrm{vgl}$. Störig 1992, 387ff 
Abb. 1: Empirismus, Rationalismus, Kant und Hegel

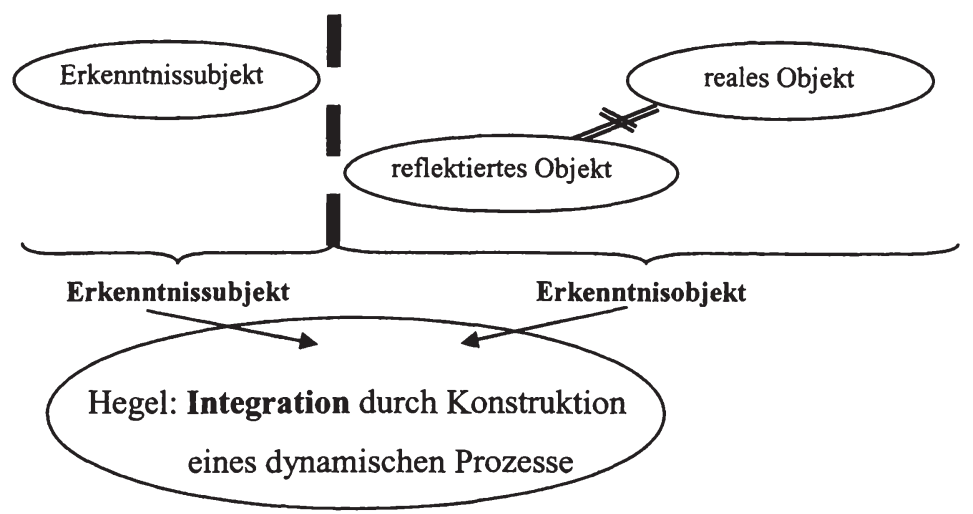

Quelle: Eigene Darstellung in Anlehnung an Schülein 2002, 86.

Die Kant'sche Trennung zwischen Erkenntnissubjekt und Erkenntnisobjekt wird von Hegel wieder aufgehoben. Für ihn ist Erkenntnis ein dynamischer, nicht linearer Prozess, der den Weg des absoluten Geistes, gedacht als permanenten Fortschritt, zu Selbstverwirklichung beschreibt. ${ }^{19}$ Diesen Prozess stellt Hegel in einem dreistufigen Modell $^{20}$ dar:

1. Position / These 1: Eine bestimmte Entwicklungsstufe des Seins

2. Negation /Antithese 2: Kritik / Zerstörung / Zerfall der These

3. Negation der Negation / Synthese: Entwicklung einer neuen Position / These 3, die die wichtigen und bleibenden Elemente der These 1 enthält, aber auch die Antithese 2 antizipiert. Der Prozess beginnt von Neuem...

Der Gesamtprozess besteht aus Teilprozessen. Diese Teilprozesse müssen durch ein vernetztes Gesamtsystem hindurch. Dabei werden sie in andere Formen und Prozesse umstrukturiert und übersetzt. Die Teilprozesse stehen in gegenseitiger Abhängigkeit. Wirklichkeit wird so zu einem dynamischen, widersprüchlichen Prozess, dessen Teilprozesse sich gegenseitig bedingen und bestimmen (dialektischer Prozess). Nichts ist, sondern alles wird. Das Jetzt ist ein nur scheinbar statischer Zustand, eine von unzähligen möglichen Momentaufnahmen eines Prozesses aus einer von unzähligen möglichen Perspektiven. Im Zuge dieses Prozesses haben daher nicht Inhalte oder Zustände Bestand, sondern nur das Gemeinsame, 
das all diesen Zuständen eigen ist. ${ }^{21}$ Darin sieht Hegel auch die Wahrheit: nicht in der scheinbaren Unmittelbarkeit der Momentaufnahme unserer Sinne, sondern in deren Bedingungen. ${ }^{22}$ Also ist Erkenntnis unabhängig von der konkreten Erfahrung. Konkrete Erfahrung ist auf Grund der Dynamik des Prozesses auch nicht möglich. Wahrheit ist selbst Teil des Prozesses. ${ }^{23}$

Ziemlich zeitgleich mit Hegels Wirken führt die industrielle Revolution zur Herausbildung eines Subsystems „Wissenschaft“. Damit war auch die Entstehung eines neuen Bildungstyps verbunden. An die Stelle des universal gelehrten Bürgers tritt nun ein speziell geschulter hauptberuflicher Profiwissenschafter, der im Rahmen der Universität durch gezieltes (methodisches) Vorgehen Forschung betreibt. Diese Technisierung der Erkenntnis führt zu einer Explosion neuen Wissens, gleichzeitig wird alles Bisherige als nicht mehr zeitgemäß verworfen. ${ }^{24}$

\subsection{Positivismus}

Die neuen Wissenschafter brauchen einfachere, prägnantere Aussagen über die Möglichkeit einer Erkenntnis, als sie die Systeme von Kant oder Hegel boten. Thre Leitwissenschaft wurde der Positivismus, der alles aus dem Bereich der Wissenschaften ausschloss, was nicht rein empirische Feststellungen treffen wollte. Daraus entstand eine Theorie der mit wissenschaftlichen Mitteln vorangetriebenen Erkenntnis, die sich durch die Abgrenzung von allem Unwissenschaftlichen durch das Kriterium der positiven Begründbarkeit zu definieren versuchte. ${ }^{25}$

Der Empirist Mill unternimmt in seinem Werk „System of Logic“ den Versuch den Syllogismus ${ }^{26}$ in eine moderne Form zu bringen. Für ihn stellt Logik die geeignete Methodik dar, die begründbare Verfahren hervorbringt, welche praktische Erkenntnisse gewährleisten. ${ }^{27}$ Der Syllogismus wird bei Mill auf Deduktion reduziert. Induktive Schlüsse rechtfertigt Mill dagegen mit dem Verweis auf die „Gleichförmigkeit im Gang der Natur.“28 Aus empirischen Daten (objektiven Erkenntnissen), die aus Beobachtungen oder Experimenten gewonnen werden, kann

$21 \mathrm{vgl}$. Hegel 1989, 89

22 vgl. Hegel $1989,87 \mathrm{f}$

23 vgl. Störig 1992, $457 \mathrm{ff}$

24 vgl. Schülein 2002, 101

25 Schülein 2002, 102

26 vgl. Müller/Halder 1973, 237

27 vgl. Störig $1992,481 \mathrm{f}$

28 Schülein 2002, 103 
der Forscher unter Verwendung der Übereinstimmungs- und Differenzenmethode $^{29}$ auf allgemeine Aussagen schließen (direkte Induktion a posteriori) ${ }^{30}$. Für Mill ist auch die soziale Realität gleichförmig und kausal aufgebaut. Daher gibt es für ihn auch nur eine Art von objektiver Erkenntnis: die Erfassung von Regelmäßigkeiten und induktiven Begründungen von kausalen Zusammenhängen. Das Problem der Erkenntnisbegründung wird zu einem Problem der Methodik. Das erkennende Subjekt ist irrelevant. ${ }^{31}$

\subsection{Argumentationslinien der Positivismuskritik}

\subsubsection{Die GeISTESWISSENSCHAFTEN UND DIE „ZWEI-WELTEN-THEORIE“}

Gianbattista Vico wehrt den Angriff der Positivisten auf die sog. Geisteswissenschaften dadurch ab, dass er den Gültigkeitsbereich des positivistischen Wahrheitskriteriums auf den unerkennbaren (also nur beschreibbaren) Bereich der Welt (Schöpfung) einschränkt und den Geisteswissenschaften einen zweiten Bereich der Welt, nämlich die Geschichte als menschengemacht und daher erkennbar ${ }^{32}$ als Betätigungsfeld zuweist. ${ }^{33}$ Für die sog. „Zwei-Welten-Theorie“ formulierte Dilthey dann die grundsätzliche Differenz im Prinzip der Erkenntnis ${ }^{34}$ :

„Es geht um die Differenz im Umgang mit der Wirklichkeit: Die Naturwissenschaften behandeln die dinglichen Eigenschaften der Welt und erklären deren gesetzmäßige Ordnung. Die Geisteswissenschaften beschäftigen sich dagegen mit dem Sinn, den die Dinge im Rahmen des menschlichen Lebens haben. Diesem Sinn nähern sie sich mit Hilfe des eigenen Erlebens und dem Verstehen des in dem Geschehen objektiven Sinns. ${ }^{435} / 36$

Damit werden die Geisteswissenschaften und somit auch die Geisteswissenschafter selbst Teil ihres Untersuchungsgegenstandes, handeln also niemals voraussetzungslos. ${ }^{37}$ Dies unterscheidet sie jedoch nicht von den Formalwissenschaftern.

29 vgl. Mill 1862, 453

30 vgl. Mill 1862, 477

31 vgl. Schülein 2002, $102 \mathrm{ff}$

32 „Nur das kann erkannt werden, was einer selbst hervorgebracht hat.“(Vico 1979)

33 vgl. Schülein 2002, 107

34 vgl. Dilthey 1974, $98 \mathrm{f}$

35 vgl. Schülein 2002, 110

36 Seiffert kritisiert die Diltheys Gegenüberstellung von, erklären' auf der einen Seite und ,verstehen' auf der anderen Seite prägnant: „Zwischen ,erklären' und ,verstehen' kann es [...] gar keinen Gegensatz geben: Was ich oder andere mir ,erklärt' [haben], das ,verstehe' ich. Es ist also schief, wenn Wilhelm Dilthey und seine Nachfolger hier einen Gegensatz konstruieren wollen [...] Auch die diesem Gebrauch durchaus entsprechende Definition von ,Erklärung' in der Wissenschaftstheorie bedeutet eine unzulässige Einengung. Es ist nicht möglich, ,Erklären' und ,Verstehen' in einen Gegensatz zu bringen: was mir erklärt wird, das verstehe ich." (Seiffert 1992, 10) 37 vgl. Dilthey 1974, 166 
Was die Geisteswissenschaften aber sehr wohl von den Formalwissenschaften unterscheidet, ist das Bewusstsein über diese Problematik. Aus der bewussten Auseinandersetzung der Geisteswissenschaften mit dem Problem der nicht zu erreichenden Unabhängigkeit des Forschers vom Untersuchungsgegenstand resultierten Überlegungen über den Umgang mit dieser Tatsache, die schließlich die Notwendigkeit allgemeiner Verstehensregeln herausstellten. ${ }^{38}$

Die Hermeneutik ${ }^{39}$ als „Wissenschaft der interpretierenden Sinnvermittlung، ${ }^{60}$ versucht genau das zu bewerkstelligen. Sie ist die methodisch reflektierte Beschäftigung mit den Gegenständen, Verfahrensweisen und Rahmenbedingungen menschlichen Verstehens ${ }^{41}$ im Allgemeinen und in konkreten Verstehenssituationen. ${ }^{42}$ Dabei ist sie jedoch in einem strukturellen Problem (,hermeneutischer Zirkel") gefangen: Um einen Zugang zum Forschungsobjekt zu finden muss der Forscher von Prämissen ausgehen, die dann seinen jeweiligen Gegenstandszugang bestimmen. Je nachdem, wie dieser Zugang aussieht, fällt seine Interpretation unterschiedlich aus und damit die Ergebnisse. Das bedeutet, dass die Ergebnisse immer von den Prämissen abhängen. Wenn sowohl die Ergebnisse als auch die Interpretationsstrategie von den Prämissen abhängen, dann werden die Ergebnisse immer die Interpretationsstrategie bestätigen. ${ }^{43}$ Somit stellt sich die Hermeneutik nicht als geeigneter Kontrollmechanismus richtigen Verstehens dar. ${ }^{44}$

Es ist aber darauf hinzuweisen, dass es sich bei diesem Vorgang nicht unbedingt um einen ,hermeneutischen Zirkel“45, gedacht als sich selbst bestätigender (tautologischer) Prozess, sondern auch um eine ,hermeneutische Spirale ${ }^{\text {“46 }}$ handelt. Dabei weist Goller-Schreckenberg auf zwei wesentliche Aspekte hin: den

38 vgl. Dilthey 1974, 167

39 Hermeneutik: 1. Etwas aussagen/ausdrücken, darstellen. 2. Etwas Gesagtes auslegen/erklären. 3. Etwas Gesagtes übersetzen/dolmetschen. $\mathrm{Zu}$ den Bedeutungen der einzelnen Wörter siehe (Seiffert 1992, 9ff).

40 Seiffert/Radnitzky 1994, 128

41 „Beim hermeneutisch relevanten Verstehen [...] kann [...] keine Rede von ,Eindeutigkeit' im Sinne des pragmatischen bewährten Alltagsverstehens sein. [...] Die eigentlich hermeneutischen Interpretationsaufgaben [...] würden gar nicht existieren, wenn sie im Sinne eines, Normalfalles' unproblematisch und eindeutig lösbar wären." (Karl-Otto Apel zitiert in: Seiffert 1992, 12)

$42 \mathrm{vgl}$. Goller-Schreckenberg 1999, 1)

43, ,.... zur Tatsache kommen wir nur durch Schlüsse, die wir aufgrund von Tatsachen ziehen müssen und umgekehrt: Schlüsse ziehen wir aus Tatsachen, zu denen wir durch Schlussfolgerungen gelangt sind.“ (Seiffert 1991 II, 104)

$44 \mathrm{vgl}$. Schülein 2002, $112 \mathrm{ff}$

45 vgl. Dilthly, $267 f f$

46 vgl. Goller-Schreckenberg 1999, 5 
Zirkel zwischen Vorverständnis und Gegenstandsverständnis ${ }^{47}$ und den Zirkel zwischen Teilen untereinander und den Teilen und dem Ganzen ${ }^{48}$. Die hermeneutische Zirkelbewegung Diltheys kann nach Danner ${ }^{49}$ auch als eine Vermittlungsbeziehung zwischen Besonderem und Allgemeinem, also zwischen Praxis und Theorie, interpretiert werden. Gadamer ${ }^{50}$ sieht

„die einzige Möglichkeit, den [...][mit der Interdependenz von Interpret, Interpretationstechnik und Interpretationsergebnis] verbundenen Problemen zu begegnen [...][in einer] Art von Dokumentation der Geschichte von Voraussetzungen und Interpretation: Durch Reflexion der Umstände, durch die eine Interpretation zustande kommt, und die Art ihrer Wirkung ergibt sich die Möglichkeit einer Interpretation der Interpretation, die ihre Begrenzungen relativiert.“51

\subsubsection{IDEOLOGIEKRITIK}

Marx erkennt als erster die Ökonomie als Motor der Geschichte und entmystifiziert damit den „objektiven Geist“ Hegels. Außerdem hält er fest, dass die Vorstellungen, die sich die Menschen von der Welt machen, Selbstinterpretationen sind und nicht eine Ausformung des ,objektiven Geistes“ Hegels sein können („Das Sein bestimmt das Bewusstsein“) $)^{52}$, denn wo kämen sonst die falschen Vorstellungen der Menschen her $^{53}$ ? Damit werden die Vorstellungen, welche die Menschen von der Welt haben, nicht nur täuschungsanfällig, sondern manipulierbar. ${ }^{54}$ Beides zusammen ergibt: Ist die Wirklichkeit, so wie es die Positivisten postulieren, durch Kontakt mit der Welt erkennbar, so kann das Erkannte über

47 Unter dem Zirkel zwischen Vorverständnis und Gegenstandsverständnis ist zu verstehen, dass der Interpret mit einem gewissen begrenzten Vorverständnis, das aus seinem wirkungsgeschichtlich und historisch bedingten Vorurteilshorizont besteht, an den Gegenstand (z.B. Text) herangeht und an ihm ein erstes Textverständnis (eine erste provisorische textbezogene Auslegungsversion) entwickelt. Die gewonnenen Einsichten erweitern nun den Vorurteilshorizont und erweitern so die Grenzen des Bewusstseins. Damit ist der Blick auf weitere Aspekte eröffnet. Das Vorverständnis wird so auf eine höhere Ebene transportiert und der Blick des Interpreten wird schärfer und umfassender. Jetzt kann der Interpret mit einem erweiterten Vorverständnis und dem Blick auf neue Aspekte erneut an den Text herangehen. Durch Wiederholung dieses Vorganges kann ein immer verbessertes Textverständnis erzielt werden. Es handelt sich dabei nicht um eine Addition von weiterem Wissen, sondern der Interpert deutet alles auf der Grundlage der neuen Basis. (vgl. Goller-Schreckenberg 1999, 3)

48 Der Zirkel zwischen Teilen untereinander und den Teilen und dem Ganzen ist zu verstehen als ein wiederholtes Abgleichen und „In-Beziehung-Setzen“ mit dem Ziel über ein besseres Verständnis des Untersuchungsgegenstandes (zB des Textes) zur Widerspruchsfreiheit der Teile untereinander und im Bezug zum Ganzen zu gelangen. (vgl. Goller-Schreckenberg 1999, 3)

49 vgl. Danner 1998, 59 f

50 vgl. Gadamer 1960

51 vgl. Schülein 2002, 115

52 vgl. Marx 1969, Bd.21, $296 \mathrm{f}$

53 vgl. Marx 1969, Bd. 21, 297

54 vgl. Marx 1969, Bd.3, 27 
eine Manipulation der Erkennenden gesteuert werden. Der Positivismus ist daher für Marx ideologieanfällig. Das menschliche Denken ist somit von gesellschaftlichen Verhältnissen gesteuert. Der Positivismus verstärkt tendenziell das, was ist.

\subsubsection{KRITISCHE THEORIE}

Die Kritische Theorie entwickelt keine eigene alternative Methodik, sondern kritisiert die des Positivismus: „Der Positivismus versteht sich [...] als arbeitsteiliges Rad im Getriebe und fügt sich mehr oder weniger blind den gesellschaftlichen Vorgaben. ${ }^{\text {" } 55}$ Denn so Horkheimer:

„Ob das Auffinden neuer Varietäten auf einzelnen Gebieten der anorganischen oder organischen Natur [...] zur Änderung alter Klassifikationen oder zum Entstehen neuer den Anlass bildet, lässt sich keineswegs nur aus der logischen Situation ableiten, hängt in Wahrheit nicht bloß von der Einfachheit und Folgerichtigkeit des Systems, sondern unter anderem auch von Richtung und Ziel der Forschung ab, die aus ihr selbst weder zu erklären noch letztlich einsichtig zu machen sind. ${ }^{56}$

Die Beziehung von Hypothesen auf Tatsachen vollzieht sich schließlich nicht im Kopf der Gelehrten, sondern in der Industrie. “" ${ }^{\text {(57 }}$ Selbst stellt die Kritische Theorie höhere Ansprüche an eine Wissenschaftstheorie. Sie sieht sich dem Ziel der Aufklärung verpflichtet und will einen vernünftigen Zustand herstellen. Dies soll nur mittels einer holistischen Sichtweise möglich sein, die Zusammenhänge von Erkenntnis und Gesellschaft stets im Auge behält. ${ }^{58}$

\subsubsection{ANALytische Philosophie}

Die Vertreter der Analytischen Philosophie interessierte weniger die Frage, ob Erkenntnis möglich ist, sondern vielmehr die Frage der Formulierung der Erkenntnis. Die Analytische Philosophie ist somit Sprachphilosophie. Innerhalb dieser Denkrichtung etablierten sich zwei konträre Programme die im Folgenden in tabellarischer Form gegenüber gestellt werden:

55 Schülein 2002,125

56 Horkheimer 1968, 144

57 Horkheimer 1968, 145

58 Schülein 2002, $125 \mathrm{f}$ 
Abb. 2: Programme der analytischen Philosophie

\begin{tabular}{|c|c|}
\hline Russel-Programm & Programm des G. E. Moore \\
\hline $\begin{array}{l}\text { Schaffung einer Kunstsprache, die Aussagen } \\
\text { ermöglicht, die von den Zweideutigkeiten } \\
\text { und Unzulänglichkeiten der Alltagssprache } \\
\text { befreit sind. }\end{array}$ & $\begin{array}{l}\text { Reduzierung der Umgangssprache auf ihren } \\
\text { unproblematischen Kern (Fachsprache), in der } \\
\text { Verständigung möglich ist. }\end{array}$ \\
\hline Wittgenstein 1 & Wittgenstein 2 \\
\hline $\begin{array}{l}\text { „Was sich überhaupt sagen lässt, lässt sich } \\
\text { klar sagen; und wo man nicht reden kann, } \\
\text { darüber muss man schweigen."61 } \\
\text { Sinnvolles Reden ist nur möglich über } \\
\text { Dinge, die der Erfahrung zugänglich sind. } \\
\text { Also Sätze mit empirischem nicht aber } \\
\text { normativem Gehalt. Dieser Versuch } \\
\text { scheiterte jedoch an der Unmöglichkeit, } \\
\text { Sätze zu formulieren die diese Vorraus- } \\
\text { setzungen erfüllen. }\end{array}$ & $\begin{array}{l}\text { Da die Wahrheit nicht an Grammatik festgemacht } \\
\text { werden kann (Scheitern Wittgenstein 1), gibt es } \\
\text { keine objektive Wahrheit. }{ }^{63} \text { Die Wahrheit ist so } \\
\text { wie die Sprache an Handlungen gebunden, also } \\
\text { an Praxissysteme. }{ }^{64} \text { Jedes Praxissystem hat sein } \\
\text { eigenes Sprachspiel }{ }^{65} \text { (Spezialsprache). So gut } \\
\text { sich die Elemente innerhalb eines Praxissystems } \\
\text { verstehen, so unmöglich ist Verstehen über die } \\
\text { Grenzen zweier Praxissysteme hinweg. }{ }^{66}\end{array}$ \\
\hline \multicolumn{2}{|c|}{$\begin{array}{l}\text { Statt die gesamte Alltagssprache auf ihren wahrheitsfähigen Kern zu reduzieren, } \\
\text { begnügt sich Wittgenstein mit der Reduzierung der Gültigkeit ihres Wahrheitsan- } \\
\text { spruchs auf das jeweilige Subsystem. }\end{array}$} \\
\hline $\begin{array}{l}\text { Wiener Kreis }{ }^{67} \text { versucht wahrheitsfähige } \\
\text { Protokollsätze zu formulieren }{ }^{68} \text {, scheitert } \\
\text { aber daran, dass keine wertfreie Aussage } \\
\text { möglich ist. }\end{array}$ & $\begin{array}{l}\text { - Relativismus } \\
\text { - Konstruktivismus }\end{array}$ \\
\hline
\end{tabular}

Quelle: Eigene Darstellung

59 Schülein 2002, 130

60 Schülein 2002, 130

61 Wittgenstein 1989, 9

62 vgl. Schülein $2002,131 \mathrm{ff}$

63 vgl. Wittgenstein 1989, 245

64 „Eine Sprache vorstellen heißt, sich eine Lebensform vorstellen.“ (Wittgenstein 1989, 246)

65 „Der ganze Vorgang des Gebrauchs der Sprache [ist] eines jener Spiele, mittels welcher Kinder ihre Muttersprache lernen. Ich will diese Spiele ,Sprachspiele' nennen. [...] Ich werde auch das ganze: der Sprache und der Tätigkeiten, mit denen sie verwoben ist, das ,Sprachspiel' bezeichnen." (Wittgenstein 1989, 241)

$66 \mathrm{vgl}$. Wittgenstein $1989,252 \mathrm{f}$

67 vgl. Störig 1992, $676 \mathrm{ff}$

68 vgl. Carnap 1932, 220

69 Relativismus bezeichnet die Anschauung, dass es eine absolute, umfassende Wahrheit für den Menschen nicht gibt und dass alles Erkennen vielmehr bezogen ist auf und abhängig ist von veränderlichen Bedingungen. (Müller/Halder 1972, 229) 


\subsection{Kritischer Rationalismus}

Einen völlig neuen Ansatz stellt Popper vor. Er ersetzt das Verifizierbarkeitskriterium des Positivismus durch das Falsifikationsprinzip ${ }^{70}$. Er argumentiert, dass die Wahrheit göttlich und daher für den Menschen nicht zugänglich ist. ${ }^{71}$ Somit müssen wir uns mit relativen Wahrheiten begnügen, von denen wir aber mittels des Falsifikationsprinzips jeweils die beste (nächste zur Wahrheit) auswählen können. An Stelle der absoluten Wahrheit, tritt die bewährte Wahrheit bis diese falsifiziert und durch eine neue, wie Popper annimmt, bessere ersetzt wird. $^{72}$ Mit diesem Kunstgriff eliminiert Popper gleichzeitig das Induktionsproblem ${ }^{73}$. Denn wenn eine Wahrheit nur so lange Gültigkeit beansprucht, bis ein Fall eingetreten ist, der sie falsifiziert, braucht sie auch nicht allgemeine Gültigkeit beanspruchen. (Der erste schwarze Schwan falsifiziert die Theorie, wonach alle Schwäne weiß sind.)

\subsection{Kritik des Kritischen Rationalismus}

\subsubsection{NEUE IDEOLOGIEKRITIK}

Der Kritische Rationalismus ist, auch wenn das Wahrheitskriterium, und damit auch der Wahrheitsbegriff, ein relativierter ist, vom Positivismus nicht genügend verschieden, um nicht dieselben Ansatzpunkte für Kritik aufzuweisen. So wundert es kaum, dass der Kritische Rationalismus einer erneuerten Ideologiekritik ausgesetzt ist. Wie dem Positivismus wird auch dem Kritischen Rationalismus vorgeworfen, ein viel zu enges, zu einseitiges und vor allem ein politisch verzerrtes Bild der Wirklichkeit zu vertreten. ${ }^{74}$

\subsubsection{FEHLER DES FORTSCHRITTSGLAUBENS}

Einen anderen Aspekt des Kritischen Rationalismus kritisiert Kuhn. Er setzt dort an, wo Popper eine permanente Verbesserung des Wissens durch permanente Kritik und rationale, begründbare Auswahl der Theorien als linearen Prozess skizziert. Für Kuhn ist die Theorie des stetigen Fortschritts der Wissenschaft ein Mythos. Seine Vorstellung von der Funktionsweise der Veränderung der Wissen-

70 Zahar, E., G. in: Keuth 1998, 103

71 „Nur die Götter besitzen Wissen; wir Sterblichen können nur vermuten und raten.“ (Popper 1995, 63)

72 vgl. Waschkuhn 1999,7

73 vgl. Seiffert 1991, 153ff

$74 \mathrm{vgl}$. Habermas 1973 
schaft über die Zeit beschreibt er anhand einer Abfolge typischer Phasen und des Konzepts des Paradigmas ${ }^{75}$. Der typische Phasenverlauf ${ }^{76}$ ist folgender:

1. Vorparadigmatische Phase: ein mehr oder weniger gleichberechtigtes Nebeneinander loser, improvisierter, konkurrierender Ansätze.

2. Paradigmatische Phase: Ein Ansatz setzt sich durch und wird allgemein anerkannt. Das herrschende Paradigma ist jetzt der Bezugsrahmen der Forscher eines Fachs. Es grenzt seine Konkurrenten aus, indem es definiert, was ,normale“ Wissenschaft ist.

3. Kritische Phase: Der Gehalt des herrschenden Paradigmas ist erschöpft. Es häufen sich Probleme, Fehlschläge und Anomalien, die den Glauben an das herrschende Paradigma erschüttern.

4. Revolutionäre Phase: Es kommt $\mathrm{zu}$ breitem Widerstand gegen das herrschende Paradigma. Alternativen werden propagiert und treffen auf verzweifelten Widerstand der Vertreter des ,alten“ Paradigmas.

5. Neue Paradigmatische Phase: Ein alternatives Paradigma erringt die Oberhand und steigt zum neuen herrschenden Paradigma auf. Vertreter des alten Paradigmas passen sich an oder sterben aus.

Der Übergang von einem Paradigma auf ein neues beruht weder auf Falsifikation noch auf Verifikation, sondern ist eine Entscheidung der Forschergemeinde zwischen inkommensurablen ${ }^{77}$ Alternativen. Die Alternativen entziehen sich einem Vergleich. Daher ist es nicht möglich zu entscheiden, welches Paradigma das bessere ist. Die Entscheidung für ein Paradigma kann somit nicht logisch begründet (bewiesen) werden, sondern ist eine Frage der Durchsetzungskraft der „neuen Forschergeneration“78. Somit gibt es auch keinen definitiven Fortschritt.

Kuhn beantwortet die Frage nach der Ursache der fälschlichen Auffassung der Geschichte der Wissenschaft als linearen Prozess mit dem Hinweis auf die Tatsache, dass Lehrbücher ständig umgeschrieben werden, so dass sie immer auf das herrschende Paradigma zentriert sind und so der jeweils herrschende Zustand

75 Paradigma im Kuhn'schen Sinn: Ontologische, methodologische und theoretische Leitvorstellungen und Leitkonzepte.

76 vgl. Schülein 2002,154f

77 Paradigmen sind inkommensurabel in dem Sinne, dass sie weder kompatibel noch verknüpfbar sind. Unter ihnen ist kein Austausch möglich. Sie existieren in verschiedenen Welten, können nicht koexistieren, schließen einander also aus.

78 Bei den Forschern, die das neue Paradigma vertreten, handelt es sich „gewöhnlich um Männer, die so jung oder auf dem von der Krise befallenen Gebiet so neue sind, dass ihre Arbeiten sie weniger tief als die meisten ihrer Zeitgenossen an die durch das alte Paradigma bestimmten Weltauffassungen und Regeln gebunden hat.“ (Kuhn 1969, 139f) 
der Wissenschaft als unvermeidbare Konsequenz dessen erscheint, was vorher geschah. $^{79}$

\subsubsection{THEORIE UND WISSENSCHAFTSPRAXIS}

Aus einer ähnlichen Richtung wie Kuhn greift auch Knorr-Cetina den Positivismus und somit auch den Kritische Rationalismus an. Sie kritisiert heftig das idealisierte Schema der Ableitung von Hypothesen aus Theorien und deren rigorose Überprüfung an der Wirklichkeit. Ihrer Ansicht nach hat dieses Verständnis von Wissenschaft wenig mit der realen Wissenschaftspraxis zu tun. Vielmehr ist Forschung ein permanenter Entscheidungsprozess ${ }^{80}$, wobei die Entscheidungen wesentlich von nicht sachlichen Themen ${ }^{81}$ mitbestimmt werden, aber gleichzeitig Einfluss auf Richtung und Art der Erkenntnis nehmen. Diese Einflüsse werden dann bei der Darstellung der Ergebnisse ausgeblendet, sodass der Eindruck einer geradlinigen Forschungstätigkeit entsteht, bei der unter Verwendung neutraler Methoden objektive Ergebnisse ermittelt werden. ${ }^{82}$ „Die Trennung von Methode und Resultat suggeriert eine Unabhängigkeit, die so nicht existiert. ${ }^{\text {" }}{ }^{83}$ Werden die Ergebnisse publiziert, so kommt es zu einer weiteren Distanzierung von den eigenen Entscheidungen z.B. durch Zitate:

„Die Endfassung argumentiert [...] zu Gunsten der vorgeschlagenen Alternative, aber sie gibt nicht länger zu, den Vorschlag gemacht zu haben. [Man bedient sich] der Guerilla-Taktik, die durch literarische Zerstreuungsstrategien verdeckt wird. ${ }^{184}$

Knorr-Cetina fordert daher, dass Ergebnisse wissenschaftlicher Forschung immer als Resultate praktischer Entscheidungen und damit vor dem Hintergrund der Bedingungen, unter denen diese getroffen wurden, gesehen werden. „Sie ergänzte die Kuhn-Kritik durch die Verdeutlichung, dass auch im Alltag die propagierte bzw. geforderte methodische und theoretische Strenge des Kritischen Rationalismus ein Mythos ist. “85

79 vgl. Schülein 2002,154f

$80 \mathrm{vgl}$. Knorr-Cetina1984, $25 \mathrm{ff}$

81 Verfügbare Technik, verfügbare Materialien, soziale Abhängigkeit, Karriereinteressen...

82 „Im wissenschaftlichen Papier hat die experimentelle Methode keine eigene dynamische Struktur: Es gibt keine Probleme, keine Ressourcen, die diese Probleme in Lösungen überleiten, keine Interessensfusionen, die die Ausführung der technischen Operationen tragen könnten." (Knorr-Cetina 1981, 212)

83 Schülein 2002, 161

84 Knorr-Cetina 1981, 230

85 Schülein 2002, 162 


\subsubsection{BEFREIUNG VOM POSITIVISMUS}

Den dritten grundsätzlichen Ansatzpunkt der Positivismuskritik formuliert beispielsweise Feyerabend, wenn er den Verhaltenskodex des Positivismus als zu simpel und disziplinierend kritisiert, als dass dieser zur Kreativität anregen könnte. Für Feyerabend wirken die Regeln, die das verzerrte positivistische Weltbild beherrschen,

„verdummend und widersprechen dem eigentlichen Ziel der Wissenschaft. Sie funktioniert dann, bleibt aber unproduktiv und weiß dies nicht einmal." ${ }^{46}$ Der Forscher gehorcht „wie ein gut dressiertes Haustier [...] dem Vorstellungsbild seiner Herren, er hält sich an die Grundsätze des Argumentierens, die er gelernt hat [...], und er kann überhaupt nicht erkennen, dass das, was er als die ,Stimme der Vernunft' ansieht, nicht anders ist als eine kausale Nachwirkung seines Trainings." ${ }^{87}$

Tatsächlich aber ist für Feyerabend die Reglementierung der Forschungstätigkeit nur eine Befriedigung des niedrigen menschlichen Bedürfnisses nach Sicherheit und führt unweigerlich zu dürftigen eindimensionalen Ergebnissen. Für ihn gibt es ,nur einen Grundsatz [...], der sich unter allen Umständen und in allen Stadien der menschlichen Entwicklung vertreten lässt. Es ist der Grundsatz: „Anything goes (Mach, was du willst) ${ }^{488}$

\subsubsection{WISSENSCHAFTSKRITIK}

Die vierte grundsätzliche Kritik geht über die drei oben dargestellten bei weitem hinaus. Während Kuhn in der Annahme, es gäbe einen linearen, qualitativen Fortschritt der Wissenschaft, einen Irrtum sieht, Knorr-Cetina die Vorstellung eines geordneten zielgerichteten, rationalen Forschungsprozesses mit von den verwendeten Methoden unabhängigen Ergebnissen bricht und Feyerabend auf die dramatischen Auswirkungen der enormen Regelungsdichte positivistischer Forschungstätigkeit hinweist, stellt die sogenannte Wissenschaftskritik die positivistisch betriebene Wissenschaft als Ganzes in Frage. Dabei wird darauf hingewiesen, dass Wissenschaft in Abhängigkeit von Geld (Wirtschaft) und Politik Produkte hervorbringt, die zwar funktional für bestimmte Interessen und Interessensgruppen, aber gleichzeitig für andere düsfunktional und für das gesellschaftliche Ganze sogar gefährlich sind. Der Grund dafür wird darin 
gesehen, dass positivistisch betriebene Wissenschaft weder einem vernünftigen Plan folgt noch imstande ist, ihre Folgen zu begreifen und zu kontrollieren ${ }^{89}$.

„Je weiter Erkenntnis sich ausdehnt, desto problematischer wird ihre Anwendung. Einerseits weil viele Chancen, die sie bietet, mit Risken verbunden sind, andererseits jedoch vor allem, weil viele Erkenntnisse hoch spezieller Art und unmittelbar nur auf bestimmte Teile von Realität bezogen sind. Ihre Anwendung ist jedoch immer eine Intervention in ein komplexes System, was zur Folge hat, dass mehr betroffen wird, als intendiert ist und das System auf komplexe, nicht lineare Weise reagiert. [...] Es besteht also in modernen Gesellschaften eine systematische Diskrepanz zwischen der Partikularität von Erkenntnis und der Systematik ihrer Anwendung.“ 90

Dem Positivismus wird zur Last gelegt, dass dieser wesentliche Themenkomplex sowie die Frage der Verteilung von Erkenntnis ${ }^{91}$ gänzlich ausgeblendet wird. ${ }^{92}$

\subsection{Alternativen zum Positivismus}

Trotz der vielen identifizierbaren Strömungen, in die sich die Opposition gegen den Positivismus mittlerweile ausdifferenziert hat, ist es nicht gelungen, eine einheitliche Gegenposition zur einfachen Prägnanz des Positivismus und des Kritischen Rationalismus zu formulieren. Einige alternative Programme zum Positivismus werden im Folgenden kurz vorgestellt.

\subsubsection{PRAGMATISMUS}

Der Pragmatismus spielte eine zentrale Rolle bei dem Versuch, Theorie stärker an Praxis zu binden und daraus Begründungen zu entwickeln. C. S. Peirce, einer der wichtigsten Erkenntnistheoretiker des Pragmatismus, betonte als anthropologische Begründung von Forschung die Tatsache, dass Zweifel ein unangenehmer Zustand sei, den der Mensch durch Forschung zu überwinden trachte. Das Ziel ist, zu Überzeugung zu gelangen, die es dann in weiterer Folge erlaubt, Verhaltensregeln ${ }^{93} \mathrm{zu}$ entwickeln. Für Peirce ist Forschung ein Mittel zur Überwindung von

89 Siehe dazu genauer Hermann 1984

90 Schülein 2002, 168

91 Dies bezieht sich auf die Frage, wem Forschungsergebnisse zugänglich sind. „Es gibt Schätzungen, die davon ausgehen, dass inzwischen etwa die Hälfte aller neuen Erkenntnis nicht mehr der (wissenschaftlichen) Öffentlichkeit frei zugänglich sind, sondern geheim gehalten oder privatisiert sind."(vgl. Schülein 2002, 167)

92 vgl. Störing, 1992, 585ff

93 Verhaltensregel ermöglichen einemn unter gegebenen Umständen bestimmte Ziele zu erreichen. 
Unsicherheit ${ }^{94}$, indem sie praktische Orientierung bietet. Denken ist für Peirce die Herstellung von praktischen Wirkungen. Er formuliert die praktische Maxime der Erkenntnis:

„Überlege, welche Wirkungen, die denkbarerweise praktische Relevanz haben könnten, wir dem Gegenstand unseres Begriffs in unserer Vorstellung zuschreiben. Dann ist unser Begriff dieser Wirkungen das Ganze unseres Begriffs des Gegenstandes. “95

Das bedeutet, dass wir in praktischen Wirkungszusammenhängen denken. ${ }^{96}$

\subsubsection{INTERPRETATIVES PARADIGMA}

Aufbauend auf den Ergebnissen der Arbeit von Schütz ${ }^{97}$ zielen die interpretativen Ansätze in eine ähnliche Richtung wie die Positivismuskritik. Schütz hatte untersucht, wie sich Menschen in der Welt orientieren. Er kommt zum Ergebnis, dass unser Alltagsbewusstsein aus der Fülle der vorhandenen Reize nur einige wenige heraushebt und zu einem sinnvollen Bild von Realität verbindet. Somit wird Wirklichkeit nicht abgebildet, sondern aktiv erzeugt. Das Alltagsbewusstsein lässt sich dabei von den lebensgeschichtlich erworbenen Vorstellungen leiten, die eng mit den gesellschaftlichen Vorgaben verflochten sind. Auch Wissenschaft ist demnach ein aktiver Erzeugungsvorgang, der sich zwar methodisch kontrolliert vollzieht, von der alltäglichen Orientierung in der Welt aber nicht systematisch verschieden ist. Es wird somit auch Wissenschaft als ein Akt des Verstehens gesehen. ${ }^{98}$ „Verstehende Ansätze [sind], wissenschaftstheoretisch gesehen, keine Ergänzung, sondern eine Alternative zu allen nicht-verstehenden Richtungen.“99

94 ,Die Tätigkeit des Denkens [wird] durch den Reiz des Zweifels ausgelöst und [...] hört [auf], wenn eine Überzeugung erreicht ist; so dass die Herstellung der Überzeugung die einzige Funktion des Denkens ist." (Peirce 1976, 187)

95 Peirce 1976, 195

Zur Veranschaulichung dieser Regel gibt Peirce Beispiele an, von denen an dieser Stelle eines dargestellt wird: Wir „wollen [...] fragen, was wir meinen, wenn wir ein Ding hart nennen. Offensichtlich dies: dass es von vielen anderen Substanzen nicht gereizt werden wird. Der ganze Begriff dieser Eigenschaft, wie der jeder anderen, liegt in ihren gedachten Wirkungen. Es gibt absolut keinen Unterschied zwischen einem harten und einem weichen Ding, solange sie nicht auf die Probe gestellt worden sind.“(Peirce 1976, 195)

96 vgl. Schülein 2002, $171 \mathrm{f}$

97 Näheres zu Alfred Schütz siehe (Grathoff 1978)

98 vgl. Schülein 2002, $173 \mathrm{f}$

99 Hitzler, R. in: Jung/Müller-Doohm 1993, 232 


\subsubsection{RADIKALER KONSTRUKTIVISMUS}

Der Radikale Konstruktivismus verabschiedet sich nun dezidiert vom Objektivitätsanspruch der Wirklichkeit. ${ }^{100}$ Ernst von Glasersfeld stellt fest: „Was immer wir unter ,Erkenntnis' verstehen wollen, es kann nicht mehr die Abbildung oder Repräsentation einer vom Erleben unabhängigen Welt sein.“101 Unser begriffliches Wissen besteht aus Modellen. Diese befähigen uns zur Orientierung in der Erlebniswelt, zur Vorhersage von Situationen und fallweise sogar zur Bestimmung von Erlebnissen. ${ }^{102}$

„Die Rolle der Wissenschaft [besteht] nicht darin, objektive Realität widerzuspiegeln, sondern darin, uns zu befähigen, in unserer Erlebniswelt zu handeln und Ziele zu erreichen. Daher rührt der [...] Grundsatz, dass Wissen passen, aber nicht übereinstimmen muss. “103

Für die Vertreter des radikalen Konstruktivismus sind Theorie und Welt absolut getrennt. Damit erteilen sie jeder Möglichkeit empiristisch fundierter Erkenntnis eine massive Absage:

„Die Annahme, dass unsere Sinne uns irgend etwas Objektives aus der ontischen Welt übermitteln können, wird hinfällig. [Es zeigt sich], dass alle Kenntnis in der Erlebniswelt konstruiert werden muss, sich ausschließlich auf eben diese Erlebniswelt bezieht und keinerlei ontologische Ansprüche auf Objektivität erheben kann. “ 104

Humberto Maturana hat diese völlige Trennung zwischen Welt und Erkenntnis ${ }^{105}$ mit dem U-Bootgleichnis ${ }^{106}$ veranschaulicht. Demnach ergeht es dem Menschen in seiner Umwelt wie einer U-Bootbesatzung. Zur Orientierung können nur die Informationen der Instrumente herangezogen werden, diese sagen aber nichts Wahres über die Umwelt aus. Solange sich der Mensch mittels der Informationen der Instrumente erfolgreich in seiner Umwelt orientieren kann, das U-Boot nicht verunglückt, passt ${ }^{107}$ die Theorie hinreichend. ${ }^{108}$

100 „Objektivität ist die Illusion, dass Beobachtungen ohne einen Beobachter gemacht werden können.“(Glasersfeld 1991, 17)

101 Glasersfeld 1991, 17

102 vgl. Glasersfeld 1991, 24

103 Glasersfeld 1991, 24

104 Glasersfeld 1991, 28; siehe dazu auch Kant 1991, B XIII und Kant 1991, $129 f$

„Die Annahme, dass unsere Sinne uns irgend etwas Objektives aus der ontischen Welt übermitteln können, wird hinfällig, wenn es zutrifft, dass die Signale unseres Wahrnehmungsapparates nicht einmal Gesehenes von Gehörtem oder Ertastetem unterscheiden.“(Glasersfeld 1991, 28)

105 Erkenntnis wird hier verstanden als konstruiertes Bild der Welt.

106 vgl.Maturana 1988, 25ff

$107 \mathrm{vgl}$. Glasersfeld 1991, $25 \mathrm{f}$

108 vgl. Glasersfeld 1991, 176 


\subsubsection{EVOLUTIONÄRE ERKENNTNISTHEORIE}

Die Evolutionäre Erkenntnistheorie baut auf biologischen Einsichten, vor allem auf der Evolutionstheorie Darwins, auf. Darwins Prinzip der Zweckmäßigkeit besagt, dass das Zusammenspiel von genetischer Mutation und ökologischer Selektion dafür sorgt, dass sich das am besten angepasste Lebewesen fortpflanzen kann und so als Typ überlebt. Lorenz ${ }^{109}$ übertrug dieses Prinzip auf die Entwicklung des menschlichen Erkenntnisvermögens. Demnach liegt der Anpassungsvorteil des Menschen in der höheren Komplexität der Informationsverarbeitung, die dem Menschen reflexive Verarbeitung von Wirklichkeit weit über die Grenzen der sinnlichen Wahrnehmung erlaubt. Die evolutiv entwickelte und angepasste Fähigkeit zur Erkenntnis bedeutet aber nicht, dass das, was wir als Erkenntnis betrachten, auch tatsächlich die Wahrheit ist. Vielmehr ist eine hundertprozentige Anpassung schon hinsichtlich der Heterogenität der Milieus nicht möglich. Aber auch eine optimale Anpassung findet laut Lorenz nicht statt, sondern es kommt aus ressourcenökonomischen Gründe lediglich zu einer hinreichenden Anpassung. Das hat zwei wichtige Folgen für unsere Erkenntnisfähigkeit:

1. Wir erkennen im Prinzip das für uns in unserer Lebenswelt unmittelbar Bedeutsame. Und jeder Versuch darüber hinaus Erkenntnis zu erlangen, führt unweigerlich zu unauflösbaren Aporien ${ }^{110}$.

2. Unser Denken ist niemals voraussetzungslos, da es immer schon an unsere Lebenserfordernisse gebunden ist und daher diese immer schon voraussetzt.

Damit gelingt die biologische Begründung des Kant'schen Denkens in Kategorien. Wir denken in Kategorien, wie Raum, Zeit, Kausalität, weil es für uns sinnvoll ist, nicht weil sie objektiv richtig (logisch zwingend) sind. ${ }^{111}$

$109 \mathrm{vgl}$. Lorenz 1973

110 Systemischer Konflikt (Aporie) ist ein Konflikt mit folgenden Eigenschaften: zwei einander entgegengesetzte Positionen, die beide wahr bzw. berechtigt sind, wobei beide voneinander abhängig sind. Aporien sind logisch unmöglich - und trotzdem real. Aporien kann man nicht lösen. (vgl. Beck/Schwarz 2000)

111 vgl. Schülein $2002,176 \mathrm{f}$ 


\subsubsection{GENETISCHE ERKENNTNISTHEORIE}

Diese gedankliche Richtung beschäftigt sich mit der Entwicklung des Erkenntnisvermögens eines Menschen von seiner Geburt an. Jean Piaget beschreibt basierend auf empirischen Untersuchungen,

„wie das Kind im Kontakt mit seiner Umwelt nach und nach seine kognitiven Strukturen denen der Wirklichkeit anpasst (Akkomodation) und umgekehrt die Umwelt, zB die Eltern, ihre Strukturen an diejenigen des Subjekts anpassen (Assimilation). Beide Vorgänge erzeugen ein Gleichgewicht (Äquilibration). “112

Dabei basiert die Entwicklung der Erkenntnisfähigkeit auf Erfahrung. Je stärker die sensomotorischen Fähigkeiten ausgebildet sind, umso mehr Erfahrungen sind möglich, und das Kind entwickelt sich von der konkreten Phase bis zur formaloperativen Stufe. Piaget geht davon aus, dass die Entwicklung der Wissenschaft (Erkenntnisfähigkeit der Menschheit) analog zur Entfaltung der Erkenntnisfähigkeit des Einzelnen abläuft. So war Verständnis physikalischer Bewegungen des Aristoteles ähnlich wie das eines Kind heute. ${ }^{113}$ Er geht dabei davon aus, dass der Mensch seine Schemata von einer gegebene Realität entwickelt. Das Ding-ansich ist für den Menschen jedoch nicht erkennbar. ${ }^{114}$

\subsubsection{PSYCHOANALYSE}

Im Gegensatz zu Piaget, der die kognitiven Muster und deren Entwicklung untersuchte, interessierte Freud vielmehr der psychische Sinnzusammenhang ihrer Verwendung. Für ihn ist Denken ein komplexer, nur teilweise bewusster Prozess, dessen Anfänge und Grundlagen nicht rational strukturiert sind. Die anfängliche Einheit der Erfahrung wird durch den Kontakt mit der Welt und durch die Entwicklung der Psyche aufgelöst: Real zusammenhängende Dinge werden aufgespaltet und die Erfahrungsfragmente getrennt behandelt. Im Laufe der Zeit entwickelt der Mensch im Umgang mit den Erfahrungsfragmenten verschiedene Problemlösungsmechanismen: u.a. Verleugnung, Rationalisierung, Verdrängung.

„Unter günstigen Bedingungen entwickelt sich das Erleben weiter in Richtung auf einen besseren, differenzierteren und stabileren Umgang mit der Innen- und Außenwelt, das Erleben reift in Richtung auf das Vermögen, Wirklichkeit als solche - unabhängig von eigenen Bedürfnissen, Problemen, Konflikten - wahrzunehmen und zu verarbeiten. Erfahrungen zeigen jedoch, dass die Tendenz zur Verwendung der frühen/primitiven/verzerrenden Mechanismen des Umgangs mit

112 Schülein 2002, 178

$113 \mathrm{vgl}$. Wenzel

114 vgl. Piaget 1996 und Vonèche 1985 
Wirklichkeit keineswegs verschwunden sind, dh. auch Erwachsene projezieren und spalten, leugnen und manipulieren Wirklichkeit.“115

Psychischer Stress oder eine beeinträchtigte Entwicklung verstärken die Tendenz des Menschen mit diesen verzerrenden Modalitäten $\mathrm{zu}$ arbeiten. Objektive Erkenntnis und damit auch Wissenschaft ist jedoch nur unter der Bedingung der Neutralisierung subjektiver Wahrnehmungsverzerrungen möglich. Solange aber Wissenschaft von Menschen betrieben wird, besteht immer die grundsätzliche Möglichkeit, dass Vorlieben, Ängste und Phantasien auf die Theorienbildung einwirken. Georges Devereux stellt fest, dass „dort, wo die Beziehung zum Thema affektiv aufgeladen ist oder affektive Probleme mit sich bringt, Methoden immer auch unbewusste Bewältigungsfunktion hätten. “116 So stellt sich die so oft betonte Objektivität und Nüchternheit des Vorgehens des Wissenschafters nur als Schutzmechanismus seiner eigenen prekären Identität und der der Wissenschaft dar und hilft bei der Verschleierung nicht wissenschaftlicher Motive für die Forschungstätigkeit. ${ }^{117}$

\subsubsection{SYSTEMTHEORIE}

Mit seinem Versuch, systemisches Denken auf Erkenntnisprobleme anzuwenden, fügte Niklas Luhmann eine weitere Perspektive dem Programm des NeoKonstruktivismus hinzu. Luhmann stellte fest, dass

„es [nicht] genügt [...], von der (unbestrittenen) Mitwirkung bestimmter (sprachlicher, psychologischer, sozialer) Ursachen am Zustandekommen von Erkenntnis auszugehen und daraus zu schließen, dass das Resultat Erkenntnis nichts anderes ist als eine [...] Konstruktion.“118

Luhmann fragte nach der Begründung der Wirksamkeit solcher Einflüsse. Er stellte fest, dass Systeme in einer Umwelt existieren und sich orientieren, die für sie erst durch Strukturierung mittels Beobachtung zugänglich wird. Die Systeme erzeugen ihre Umwelt erst dadurch, dass sie unterteilen und die Teile bezeichnen. Die so geschaffene Vorstellung von Realität dient dem System dann als interner Bezugspunkt für weitere Aktivitäten. Gleichzeitig entstehen die Systeme erst durch ihre Abgrenzung zu ihrer Umwelt und durch die Organisation ihrer Umweltbeziehungen. Die so gewonnenen Vorstellungen sind jedoch kein Abbild der Umwelt. Das, was sich für das System als Gegenstand darstellt, ist kein Element

115 Schülein 2002, $180 \mathrm{f}$

116 Schülein 2002, 183

117 vgl. Schülein 2002, $180 \mathrm{ff}$

118 Luhmann 1984, 521 
der Umwelt, sondern lediglich eine bezeichnete Einheit im beobachteten System. Somit ist systematische Erkenntnis (Wissenschaft) nichts anderes als eine professionalisierte Form eines alltäglichen und überlebensnotwendigen Vorganges. Wissenschaft als Operation, die organisierte Regeln be(ob)achtet, ist in dieser Perspektive Beobachtung zweiter Ordnung (ein Beobachten von Beobachten).

„Dabei sind ,Objektivität' und Wahrheit' Kategorien, mit denen das Beobachten funktioniert, aber keine wie auch immer gearteten Feststellungen über eine Übereinstimmung von Vorstellungen und Realität. “"19 
Dafür sind zwei Gründe verantwortlich:

1. Das System Mensch kann sein Gehäuse nicht verlassen. ${ }^{120}$

2. Die Beobachtung bleibt immer Teil des Systems.

Die praktische Nutzbarkeit der Erkenntnis sagt nichts über deren Richtigkeit aus, sondern zeigt nur, dass etwas machbar ist. ${ }^{121}$

„Es geht bei Technik [...] um das Ausprobieren von Kombinationsspielräumen. [...] Dass es funktioniert, wenn es funktioniert, ist [...] der einzige Anhaltspunkt dafür, dass die Realität so etwas toleriert. “"122

\subsection{Theorie und Realität}

Aus der bisherigen Darstellung ist leicht ersichtlich, dass über die Fragen der Begründung von Erkenntnis und nicht einmal über die Frage der Möglichkeit von Erkenntnis und deren Bedingungen auch nur annähernd ein Konsens erreicht wurde. Woran scheitert die Entwicklung einer gemeinsamen Basis für Erkenntnisund Wissenschaftstheorie? Dafür sind nach Schülein ${ }^{123}$ vor allem zwei Gründe zu nennen: Erstens beschäftigen sich Erkenntnis- und Wissenschaftstheorien nicht mit einer einzigen, sondern mit verschiedenen Typen von Realität. Zweitens sind Erkenntnis- und Wissenschaftstheorie selbst ein Theorientyp, der Probleme enthält, die systematisch keine Vereinheitlichung zulassen.

\subsubsection{REALITÄT - LOGISCH UNTERSCHIEDLICHE GEGENSTÄNDE}

Der auf Dilthey zurückgehende Versuch der strikten Unterteilung der Wissenschaft in Formal- und Geisteswissenschaften durch simple, sich gegenseitig ausschließende Zuweisung ganzer Wissenschaften $\mathrm{zu}$ einem der beiden Typen scheitert, da sich die Unterschiede nicht auf der gegenstandslogischen Ebene festmachen lassen. Vielmehr müssen sie auf einer abstrakteren Ebene, auf der Ebene der Logik der Realität ${ }^{124}$, getroffen werden. Es lassen sich zwei Grundtypen von Realität unterscheiden:

1. Nomologische Realität, die innerhalb gewisser Grenzen konstant und unveränderlich gegeben ist. Sie funktioniert immer und überall auf die gleiche Weise.

120 Siehe Maturanas U-Boot Gleichnis in Abschnitt 2.7.3. Radikaler Konstruktivismus.

121 vgl. Schülein $2002,184 \mathrm{ff}$

122 Luhmann 1984, 186

$123 \mathrm{vgl}$. Schülein 2002, $188 \mathrm{f}$

124 Feststellungen über die Logik des Gegenstands beziehen sich dann nicht mehr auf empirische Gegebenheiten, sondern auf deren logische Komponenten. 
2. Autopoietische Realität, die veränderlich und daher immer verschieden ist, sich selbst entwickelt und steuert und dabei mit ihrer Umwelt interagiert. Sie besitzt einen offenen Entwicklungshorizont.

\subsubsection{VON LOGISCH UNTERSCHIEDLICHEN THEORIEN ZUR FORDERUNG NACH MULTIPARADIGMATIK}

Nomologische Realität lässt sich nicht manipulieren, weil sie immer und überall auf die gleiche Weise funktioniert. Theorien, die sich mit rein nomologischer Realität beschäftigen, können diese methodisch fixieren und aus ihrem Kontext heben und in beliebige Kontexte betten. Denn das, was immer und unter allen Umständen gleich abläuft, kann von seinen jeweiligen Rahmenbedingungen getrennt und kontextunabhängig darstellen werden. Theorien, die ausschließlich nomologische Realität behandeln, sind denotativ: „eindeutig abgrenzend, zuordnend und festlegend.“125 Bei korrekter Anwendung auf den gleichen Gegenstand kommen denotative Theorien immer zu den gleichen Ergebnissen.

Autopoietische Realitäten haben eine Eigenlogik. Sie entwickeln sich selbständig und unvorhersagbar. Daher kann von Vergangenem nicht auf Zukünftiges geschlossen werden. Auswirkungen von Interventionen in solche Systeme können nicht vorausgesagt werden. Autopoietische Realität ist nicht nur eigendynamisch, sondern auch widersprüchlich. Die Vielzahl der relevanten Faktoren und ihre Variationen können theoretisch nicht erfasst werden. Auch die Kontextunabhängigkeit, die eine Darstellung der Ergebnisse eines Experiments in allgemeiner Form erst möglich macht, ist im Bereich autopoietischer Realität nicht erreichbar, da eine solche Wirklichkeit immer mit den experimentellen Arrangements interagiert und so beim Experimentieren eine eigene, spezielle Wirklichkeit geschaffen wird. Experimentieren ist mit autopoietischer Realität nur sehr eingeschränkt möglich.

„Dies hat zur Folge, dass es in Bezug auf autopoietische Realität keine eindeutigen methodologischen Verfahren geben kann, sondern immer nur Rezepte des Umgangs mit empirischer Komplexität, die unterschiedlich aussehen können. Es gibt also nicht nur eine Form der Hermeneutik, sondern deren viele; nicht nur eine Form quantitativer Sozialforschung, sondern verschiedene. Jedes dieser Rezepte hat seine Vor- und Nachteile. Das gleiche gilt für Theorien " ${ }^{\text {"126 }}$

Es sind immer verschiedene theoretische Konzeptualisierungen autopoietischer Realität möglich. Einschätzungen und Zuordnungen sind nicht eindeutig 
vorzunehmen. Sie hängen von der jeweiligen Perspektive ab, unter der man diese Realität betrachtet. Wenn im Zuge der theoretischen Konzeptualisierung (aus der bestimmten Perspektive) aus der Menge der möglichen bestimmte Einschätzungen und Zuordnungen ausgewählt werden, werden dadurch einige Aspekte der Realität betont und andere ausgeblendet. Dies ist notwendig, um das Thema stärker festzulegen, als es der Realität entspricht, und um so allgemeinere Aussagen treffen zu können, die Erklärungen ermöglichen. Die jeweilige Theorie kann somit nur eine auf die betonten Aspekte bezogene Leistung erbringen. Leistungen bezüglich der ausgeblendeten Aspekte können nicht erbracht werden. Konnotative Theorien konstruieren aktiv ihren Gegenstand.

Bei diesem Konstruktionsprozess muss ein Gleichgewicht gefunden werden zwischen Themenfixierung zur Ermöglichung von Erklärungen einerseits und Entsprechung der Theorie mit der Komplexität der Realität zur Vermeidung von Rigidität und Unproduktivität andererseits. Es gibt kein Kriterium für die Beurteilung der Güte konnotativer Theorien, diese stehen daher unter einem ständigen Legitimationsdruck. Es ist notwendig, autopoietische Realität aus immer neuen Blickwinkeln und in immer anderen Dimensionen theoretisch zu beleuchten. Nur so kann der Komplexität autopoietischer Realität entsprochen werden. Die Menge der einnehmbaren Perspektiven darf dabei nicht von den Grenzen eines Paradigmas beschränkt werden. "Multiple Thematisierbarkeit hat Multiparadigmatik zur Folge.“127

\subsubsection{ERKENNTNISTHEORIE ALS KONNOTATIVE THEORIE}

Die zweite Konsequenz der Überlegungen aus dem vorhergehenden Abschnitt betreffen die Koexistenz von denotativer Realität und konnotativer Realität und deren Mischverhältnis ${ }^{128}$ bei konkreten Problemstellungen. Ist man mit einer bestimmten Realität konfrontiert, so kann man in dieser einige Faktoren bestimmen, auf die Merkmale einer nomologischen Realität zutreffen. Diese Merkmale können dann auch aus dem Kontext, den autopoietischen Faktoren als Residualmenge, gelöst werden und mit einer denotativen Theorie und dem Prinzip des

127 Schülein 2002, 196

128 Der Begriff Mischverhältnis ist im hier verwendeten Sinn analog zur Chemie zu verstehen. Dort unterscheiden sich Mischungen von Lösungen dadurch, dass bei ersterer die einzelnen Moleküle zwar in einer Substanz vermengt sind, nicht jedoch in dieser aufgehen. Bei Lösungen hingegen hören die ursprünglichen Moleküle auf zu existieren und die Elemente verbinden sich zu neuen Molekülen. Die ursprünglichen Moleküle gehen in der Substanz auf. Realität besteht so verstanden, somit aus einer Mischung aus konnotativen und autopietischen „Molekülen“, die sich jedoch nicht verbinden sondern separat existieren. 
Experiments bearbeitet werden. Das, was für die nomologische Realität als Rahmenbedingung gilt, stellt aber gleichzeitig die autopoietische Realität dar. Die Faktoren, die jenen Teil der Realität ausmachen, können nur mit dennotativen Theorien bearbeitet werden. Das heißt, will man eine bestimmte Realität theoretisch erfassen, so steht man nicht vor einer Entscheidung zwischen den Alternativen: denotative oder konnotative Theorien. Vielmehr ist zu fragen, welche Elemente können mit welchem Theorietypus erfasst werden. So wie jede bestimmte Realität eine Komposition aus nomologischen und autopoietischen Faktoren darstellt, ist ihre theoretische Erfassung auch nur mit einer Mischung aus denotativen und konnotativen Theorien möglich.

„Die Leistung - die Kunst-, die von der Theorie verlangt wird, ist also, das jeweilige Verhältnis von denotativer und konnotativer Theoriestruktur zu finden und zu formulieren." ${ }^{129}$

\subsubsection{UNABSCHLIEBBARKEIT DER ERKENNTNISTHEORIEARBEIT}

Des weiteren lassen sich aus den vorangegangenen Überlegungen auch Gründe für die Unmöglichkeit einer endgültigen Begründung einer Erkenntnis- oder Wissenschaftstheorie ableiten. Erkenntnistheorien haben Erkenntnis zum Gegenstand. Erkenntnis ist immer eine Beziehung zwischen Sachverhalten. Diese Sachverhalte sind sowohl nomologischer als auch autopoietischer Natur. Eine Beziehung ist immer interaktiv. Das bedeutet, dass Erkenntnistheorien immer (auch) konnotative Theorien sein müssen, um dem autopoietischen Charakter ihres Gegenstandes (Erkenntnis) entsprechen zu können.

„Als Meta-Theorien [...] stehen Erkenntnis- und Wissenschaftstheorien vor dem Problem, Begründungen liefern zu müssen für eine bestimmte Form autopoietischer Realität.“130

Damit sind sie mit den Problemen konfrontiert, die für alle konnotativen Theorien Gültigkeit haben, auch mit der Unabschließbarkeit der Theorienarbeit ${ }^{131}$.

\subsection{Konsequenzen für die vorliegende Arbeit}

Wenn die Entwicklung einer Erkenntnistheorie nicht abschließbar ist, dann stellt sich die Frage, ob die eingangs geforderte Formulierung eines Fundaments wissenschaftlicher Erkenntnis überhaupt möglich ist. Versteht man unter dieser

129 Schülein 2002, 203

130 Schülein 2002, 205

131 Weil es immer noch andere Perspektiven gibt, aus denen der Gegenstand noch nicht betrachtet wurde. Man kann nie alle Perspektiven eingenommen haben, daher ist die Theorienarbeit nie abgeschlossen. 
Basis wissenschaftlicher Arbeit eine einzige, umfassende Theorie der Erkenntnis (quasi aus einem Guss), die den Bezugsrahmen für alle wissenschaftlichen Aussagen darstellt und Gültigkeitskriterien für deren Beurteilung bereitstellt, so fällt die Antwort negativ aus. Dennoch ist es möglich, ein Fundament zu schaffen, das tragfähig genug ist, um Aussagen treffen zu können, die berechtigterweise den Anspruch erheben, Erklärungen darzustellen. Dazu muss man Pfosten in den sandigen Boden der Erkenntnisbegründung treiben. Als solche Pfosten sind die Leitprinzipien zu verstehen, die hier zu formulieren und in weiterer Folge strikt einzuhalten sind. Diese Leitprinzipien sind Konsequenzen, die sich für die vorliegende Arbeit aus der Diskussion der Erkenntnisproblematik ergeben. Thre Summe ergibt das Bild der erkenntnistheoretischen Positionierung.

\subsubsection{INTERSUBJEKTIVE TRANSPARENZ UND DOKUMENTIERTE ENTSCHEIDUNGEN}

Spätestens seit Knorr-Cetina gezeigt hat, welch bestimmenden Einfluss Entscheidungen des Forschers auf den Forschungsprozess und damit auf das Forschungsergebnis haben und welche nicht wissenschaftlichen Motive hinter jeder einzelnen Entscheidung stehen (können), ist klar, dass sich diese Entscheidungen nicht zwangsläufig ergeben, sondern vom Forscher aktiv getroffen werden. Damit entsteht das Bild des opportunistischen Forschers, der durch willkürliche Entscheidungen im Forschungsprozess zu rein subjektiven Ergebnissen kommt. ${ }^{132}$

Diese höchst unbefriedigende Situation kann nicht hingenommen werden, ohne der eigenen Forschungstätigkeit selbst die Grundlage zu entziehen. Wie kann trotzdem Forschung stattfinden, die den Anspruch erhebt Aussagen zu treffen, die über den konkreten Einzelfall hinaus Gültigkeit haben? Jede Entscheidung löst Effekte aus und eliminiert andere Effekte, die von den Optionen ausgegangen wären, die nicht gewählt wurden. ${ }^{133}$ Das Treffen von Entscheidungen ist im Forschungsprozess genauso unvermeidlich wie die Beeinflussung des Entscheidungsträgers durch nichtwissenschaftliche Motive. Daher kann es nur einen möglichen Weg geben, der unter diesen Voraussetzungen dennoch Intersubjektivität der Aussagen ermöglicht:

F1: Nur durch eine möglichst vollständige Darstellung der zur Verfügung stehenden Alternativen und einer konsequenten Dokumentation der Beweggründe, die in der konkreten Situation zur einer bestimmten Entscheidung gefüht haben,

132 Siehe dazu Abschnitt 2.6.3. Theorie und Wissenschaftspraxis.

133 vgl. Fink 2002, 156 
ist es möglich, über Nachvollziehbarkeit intersubjektive Transparenz ${ }^{134}$ zu ermöglichen.

\subsubsection{DURCH GUTES ERKLÄREN VERSTEHEN SICHERN}

Wie eingangs festgestellt wurde, ist das Ziel wissenschaftlicher Arbeit, nicht nur Ergebnisse hervorzubringen, sondern diese auch an die Öffentlichkeit zu transportieren. Dabei ist man jedoch mit den Unzulänglichkeiten einerseits der Sprache und andererseits der textlichen Darstellung konfrontiert. Es ist offensichtlich, ,dass man nicht davon ausgehen kann, mit der Sprache, die verschriftlicht in die eindimensionale Buchform gepresst werden muss, Forschungsergebnisse missverständnisfrei wiedergeben zu können.“ ${ }^{\text {135 }}$ Wenn aber Verstehen möglich sein soll, muss das Augenmerk auf das Erklären gerichtet sein. ${ }^{136}$ Das bedeutet:

F2: Gedankengänge, Argumente und Ergebnisse müssen in einer klaren, nachvollziehbaren und verständlichen Weise dargestellt werden.

\subsubsection{DIE FORSCHERGEMEINDE ALS KRITISCHES KORREKTIV}

Wenn die Entscheidungen im Forschungsprozess verständlich (F2) dokumentiert werden (F1), so enthält der Endbericht des Forschungsprojektes die Entstehungsgeschichte des Forschungsergebnisses. Durch die Veröffentlichung kommt es zur Konfrontation mit der Forschergemeinde ${ }^{137}$. Diese wird, sofern die Ergebnisse des jeweiligen Forschungsprojektes durch genügend „Neuheitswert" Aufmerksamkeit erregen, reagieren, indem sie weitere Untersuchungen anstellt, um die Ergebnisse zu überprüfen. Auch wenn eine Überprüfung weder im Sinne einer Verifikation noch im Sinne einer Falsifikation abschließend möglich ist ${ }^{138}$, so trägt sie doch zur Weiterentwicklung der Theorie auf dem jeweiligen Gebiet bei. Finden Ergebnisse des Forschungsprojekts oder Teile daraus fruchtbare Anwendung in späteren Projekten, so ist das ein Indikator für die Güte der Argumente bzw. Aussagen.

134 vgl. Rößl 1993, 34

135 Rößl 1993, 34

136 vgl. Hitzler (1993), 224ff

137 Röß1 1993, 34

138 Die Falsifikation scheitert daran, dass auch eine Beobachtung, die im Popper'schen Sinn eine Theorie widerlegt, eine Theorie ist, die ihrerseits nicht Allgemeingültigkeit beanspruchen kann. Außerdem ist es denkbar, dass eine Aussage, die in einem Bereich „falsch“ ist, in einem andern ,richtig“" ist. 
F3: Die Ergebnisse des Forschungsprojekts und deren Entstehungsgeschichte müssen so veröffentlicht werden, dass sie der Kritik der Forschergemeinschaft zugänglich sind.

\subsubsection{IDEEN- UND METHODENPLURALISMUS}

Keine Theorie ist ein Totalmodell der Wirklichkeit. Ein Modell der Wirklichkeit, das alle Einzelheiten vollständig abbildet, hätte gegenüber der Realität auch keine Vorzüge. Es müssen immer Teile der Wirklichkeit ausgeklammert werden, um andere handhabbar modellieren zu können ${ }^{139}$. Dabei muss aber davon ausgegangen werden, dass jeder Aspekt der Wirklichkeit relevant ist. Gerade die Forschungsgegenstände der Sozialwissenschaften und damit auch der Wirtschaftswissenschaften stellen als autopoietische Realität besonders hohe Ansprüche an die Theorienbildung. Wissenschaftliche Arbeit im Bereich konnotativer Theorien setzt immer die Einnahme einer Perspektive voraus. Das bedeutet, dass potentiell relevante Aspekte der Realität ignoriert werden (Komplexitätsreduktion), um andere mit der begrenzten, menschlichen kongnitiven Kapazität denkbar zu machen (Modellierung). Durch diesen Reduktionismus entstehen systematisch Fehler. ${ }^{140}$ Diese Fehler sind aber nur aus einer anderen Perspektive erkennbar.

F4: Erst durch Ideen- und Methodenpluralismus können Fehler minimiert werden und Reduktionismus als Methode im Umgang mit der Komplexität der Realität gerechtfertigt werden.

\subsubsection{PRAgmatisch NÜTZLICHE AUSSAGEN}

Aus der Diskussion der Probleme der Erkenntnis ergibt sich: Da es keine endgültige Erklärung für die Möglichkeit von Erkenntnis gibt, können Theorien ihren Wahrheitsanspruch auch nicht rechtfertigen. Davon sind auch Aussagen, die auf der Basis der jeweiligen Theorie getroffen werden, erfasst. Das hat allerdings für die sozialwissenschaftliche Forschung weniger weitreichende Konsequenzen, als man vermuten mag. Wissenschaft, die das Ziel verfolgt, auf der Basis von theoretischen Konstruktionen der Wirklichkeit Aussagen zu treffen, die praktisch relevant sind, muss nicht notwendigerweise wahre und vollständige Bezugspunkte bereitstellen. ${ }^{141}$ Pragmatisch ist daher festzustellen:

139 Siehe dazu Abschnitt 2.8.1. Realität - Logisch unterschiedliche Gegenstände.

140 Siehe dazu Abschnitt 2.8.2. Von logisch unterschiedlichen Theorien zur Forderung nach Multiparadigmatik.

141 Siehe dazu Abschnitt 2.7.1. Pragmatismus. 
F5: Es sind praktisch nützliche Aussagen zu generieren, die die Wahrscheinlichkeit erhöhen, in einer Situation adäquat zu handeln. ${ }^{142}$

\subsubsection{HEURISTIK}

Das Bild der Wissenschaft, die Stein für Stein am Turm der Wahrheit baut, indem sie immer neue Teile der Wahrheit entdeckt, ist somit nicht mehr haltbar. Vielmehr ist es so, dass wir mit jedem neuen Forschungsprojekt aus einer anderen Perspektive einen anderen Aspekt des Forschungsgegenstands beleuchten, der sich aus dem methodologischen Vorgehen ergibt. Aber auch die Einsichten, die sich aus den verschiedenen Perspektiven ergeben, fügen sich nicht zusammen wie die Steine eines Puzzles, dessen Bild sich mit fortschreitender Bearbeitung immer mehr vervollständigt. Mit der theoretischen Bearbeitung sozialwissenschaftlicher Forschungsobjekte verhält es sich so wie mit dem dreidimensionalen "Magic Cube" ${ }^{143}$. Jedes Forschungsprojekt verdreht die Unterelemente gegeneinander. Das Problem dabei ist, dass durch die Perspektivität des Forschungsansatzes nur jeweils eine Seite des Würfels zu sehen ist. Das methodologische Vorgehen wird so gewählt, dass die Chancen, die sichtbare Seite in eine einheitlichen Farbe zu färben maximiert werden. Es ist offensichtlich, dass es dadurch sehr wahrscheinlich auf anderen Seiten des Würfels zu Rückschlägen kommt. Dennoch ist es möglich den „Magic Cube“ zu lösen und es gibt viele Wege zur Lösung. Im Gegensatz zum "Magic Cube“ hat man es in den Sozialwissenschaften nicht mit statischen Objekten in nur drei Dimensionen (Länge, Höhe, Tiefe) zu tun. Damit wächst die Zahl der Ebenen, die in Harmonie gebracht werden müssten, um das „Wahrheitsrätsel“ zu lösen, ins Unendliche. Die Aufgabe wird damit unlösbar. Dennoch ist das Lösungsprinzip dasselbe: Man muss den Würfel drehen (immer neue Perspektiven einnehmen) und die Unterelemente verdrehen (neue Konstellationen schaffen um die Realität neu zu konstruieren). Feyerabend formuliert: „Anything goes. (Mach was Du willst) ${ }^{\text {“144 }}$ Für die wissenschaftliche Arbeit ergibt sich daraus:

142 vgl. Rößl 1993, 35 und die dort zitierte Literatur.

143 Das im Original "Magic Cube" genannte 3D Puzzle wurde von Ideal Toy Corporation später als "Rubik's Cube" vermarktet. Jede Seite des Magischen Würfels besteht aus vier mal vier Unterwürfeln, die sich entlang von drei Achsen gegeneinander verdrehen lassen. Dabei werden alle Unterelemente, die sich in derselben Ebene befinden, mitbewegt. Die Unterelemente sind in sechs verschiedenen Farben eingefärbt. Von jeder Farbe gibt es sechzehn Unterelemente. Ziel des Spieles ist es, die Elemente so gegeneinander zu verdrehen, dass jede Seite des Hauptwürfels in einer einheitlichen Farbe erscheint.

144 Feyerabend 1993, 45 
F6: Ziel ist nicht eine objektive Realität zu erkennen, sondern jene Elemente der subjektiven Realität zu bestimmen, die als Hinweise auf relevante Wirkungsprinzipien angenommen werden. ${ }^{145}$ Die neuen Einsichten eröffnen neue Perspektiven. Das erweitert das Spektrum möglichen Handelns und damit auch möglichen Forschens.

\subsubsection{VERANTWORTLICHKEIT DES FORSCHERS FÜR „SEINE“ WIRKLICHKEITS- KONSTRUKTION}

Akzeptiert man, dass es kein Wahrheitskriterium gibt, so kann man ein Forschungsergebnis nicht damit legitimieren, dass es wahr ist. Genauso wenig gelingt mangels Nützlichkeitskriterium die a priori Feststellung der Nützlichkeit einer Aussage. ${ }^{146}$ Als einzige Möglichkeit, theoretische Konstrukte und die daraus abgeleiteten Aussagen zu legitimieren, ist die Glaubwürdigkeit des Forschers. Das Berufen auf Sachzwänge ist unzulässig, da es sich bei diesen um Denkzwänge handelt, die nur vordergründig das Verhalten des einzelnen determinieren. Das heißt für wissenschaftliches Arbeiten:

F7: Der Forscher hat für ", seine“ Konstruktion der Wirklichkeit selbst Verantwortung zu tragen.

\subsubsection{MUTIGE FORSCHUNGSFRAGEN}

Wer auf ein schnelles und möglichst sicheres Ergebnis abzielt, der stellt Fragen, die schon vorab beantwortet sind. Um Maturanas Bild vom U-Boot ${ }^{147} \mathrm{zu}$ bemühen, bewegen sich solche Arbeiten in sicherem Gewässer. Die Präzision der Instrumente ist für die durchgeführten Manöver ausreichend, und das ist vorab bekannt. Solche Arbeiten kommen fast nie zu negativen Ergebnissen, führen aber eben selten zu neuen Einsichten.

Wissenschaftliche Arbeit sollte aber auf die Generierung neuer Einsichten abzielen. Demnach wird in vorliegender Arbeit versucht, neues Gewässer zu erkunden. Dabei sollen mutige Manöver durchgeführt werden, die die Instrumente des U-Boots auf eine harte Probe stellen werden. Nur so können die Instrumente auf ihre Tauglichkeit hin getestet werden. Scheitern ist immer eine prinzipielle Möglichkeit, je mutiger und neuartiger die Manöver sind, desto höher ist diese Wahrscheinlichkeit des Scheiterns. Jedoch ist darauf hinzuweisen, dass ein 
Scheitern des Manövers auch eine wesentliche Einsicht bringt: Die Instrumente waren eine nicht hinreichend präzise Konstruktion der Wirklichkeit und müssen neu konstruiert werden.

F8: Es sollen mutige Forschungsfragen formuliert werden, die die herrschende Theorie an ihre Grenzen bringt. 



\section{Der Erkenntniszusammenhang}

Den Ausgangspunkt der theoretischen Betrachtungen für diese Arbeit bildet der Versuch den Bedeutungsinhalte des Begriffs Vertrauen zu bestimmen. Es stellt sich heraus, dass Vertrauen nur in einem Denkrahmen analysiert werden kann, in dem Situationen der Unsicherheit denkbar sind, da Vertrauen eine mögliche Reaktion der Akteure auf Unsicherheit darstellt. Des Weiteren werden zwei Arten von Vertrauen vorgestellt: instrumentelles und maximenbasiertes Vertrauen. Das Kriterium für diese Unterscheidung ist die Motivation der Akteure. Es wird dann der höhere Stellenwert des intrinsisch motivierten maximenbasierten Vertrauens erklärt. Nach einem kurzen Exkurs, der auf die Funktionsweise von Maximen eingeht, wird der Opportunismusspielraum, die Parameter seiner Bemessung und sein Verhältnis zu Unsicherheit besprochen. Auf der Differenzierung zwischen instrumentellem und maximenbasiertem Vertrauen baut sodann die Analyse der Komplexitätsbewältigungsstrategien auf. Es wird gezeigt, wie die Reduktion des Opportunismusspielraums auf instrumentellem Vertrauen basiert, während der Begrenzung der Opportunismusneigung maximenbasiertes Vertrauen zugrunde liegt. Anschließend wird auf die Selbstverpflichtung und ihre entscheidende Rolle im Entstehungsprozess eines maximenbasierten Vertrauensverhältnisses eingegangen. Danach wird gezeigt, dass ein maximenbasiertes Vertrauensverhältnis der Schlüssel für eine mögliche Lösung sozialer Dilemmata von der Art des Gefangenendilemmas sein kann. Hochgradig komplexe und unsichere Transaktionsbeziehungen, die auf eine langfristige Zielerreichung angelegt sind (Synergiesysteme), stellen solche sozialen Dilemmata dar. Somit ist Selbstverpflichtung als Erfolgsfaktor bei Synergiesystemen identifiziert.

\subsection{Von der Unsicherheit zum Vertrauen}

\subsubsection{UNBRAUCHBARE ANNAHMEN}

Lange Zeit wurde in der Fachliteratur ein Begriff relativ undifferenziert verwen$\operatorname{det}^{148}$, dessen nähere Betrachtung viel zu einem besseren Verständnis von, aus klassischer Sicht, anomalen Phänomenen beiträgt: das Vertrauen. Auch für die Behandlung des vorliegenden Themas ist eine eingehende Untersuchung des Vertrauensbegriffs unabdingbar. Die gängige Verwendung des Begriffs Vertrauen als Black Box im Sprachspiel der Ökonomie lässt den Bedeutungsinhalt jedoch weitgehend unbestimmt. Argumente, die sich auf eine bedeutungsleere Worthülse stützen, sind unangreifbar, gleichzeitig aber auch aussagelos. Will man Aussagen 
treffen, die auf dem Begriff „Vertrauen“ aufbauen, so muss man zuerst seinen Bedeutungsinhalt bestimmen. Dies ist jedoch bislang nur in unzureichender Weise geschehen. Erst in den letzten Jahren finden sich in der Fachliteratur vermehrt Anstrengungen zur Durchbrechung dieses Regressstops.

Es wird hier daher zuerst der Versuch unternommen, den Bedeutungsinhalt des Begriffs „Vertrauen“ zu bestimmen, um auf diesem Begriff aufbauend vertrauensbasierte Strategien zur Komplexitätsbewältigung besprechen zu können. Als Ausgangspunkt der Überlegungen scheint die Frage nach der Ursache der stiefmütterlichen Behandlung des Vertauensbegriffs in der älteren Fachliteratur als besonders geeignet. Es zeigt sich, dass die klassische Theorie der Ökonomie den Problemkreis Vertrauen nicht so sehr nicht behandeln wollte, sondern vielmehr auf Grund ihrer Annahmen über den homo economicus nicht behandeln konnte.

Unbegrenzte Rationalität und vollständige Information ${ }^{149}$ ermöglichen dem homo economicus sämtliche Verhaltensalternativen aller Akteure des Wirtschaftslebens, einschließlich seiner eigenen, zu kennen und zu erfassen. Während die Annahme vollständiger Information einem solchen Akteur uneingeschränkte Kenntnis über die Optionen aller Akteure unterstellt, schreibt ihm die Annahme unbegrenzter Rationalität die kognitive Kapazität $\mathrm{zu}$, diese Informationen fehlerfrei zu verarbeiten. Dem vollständig informierten und unbegrenzt rationalen Akteur sind daher auch alle möglichen Handlungsfolgen bekannt. Fügt man nun grenzenlosen Opportunismus aller Akteure als Verhaltensannahme hinzu, ergibt sich eine Situation, in der Unsicherheit denkunmöglich ist, denn die Akteure wählen aus ihren Handlungsalternativen jene aus, deren Folgen für sie am günstigsten sind. Somit ist das Verhalten aller Akteure vorhersagbar und Unsicherheit hat in diesem Denkrahmen keinen Platz. Eine theoretische Betrachtung des Phänomens Vertrauen ist aber nur in einem Denkrahmen möglich, in dem zumindest Situationen konstruierbar sind, die durch Unsicherheit über das Verhalten der Akteure gekennzeichnet sind. Der homo economicus ist sich über das Verhalten seiner Artgenossen sicher, für ihn ist Vertrauen nicht nur unnotwendig, es ist ihm gar nicht möglich zu vertrauen!

Jeder Denkrahmen, der dem Akteur vollständige Information, unbegrenzte Rationalität und unbegrenzt opportunistisches Verhalten unterstellt, operiert mit Annahmen, die für eine Betrachtung vertrauensbasierter Komplexitätsbewältigungsstrategien unbrauchbar sind. Aus diesem Grund werden sie für die weiteren, in

149 „Der völlig Wissende braucht nicht zu vertrauen [im Originaltext hervorgehoben], der völlig Nichtwissende kann vernünftigerweise nicht einmal vertrauen." (Simmel 1968, 263) 
diesem Beitrag dargestellten, Überlegungen durch unvollständige Information, begrenzte Rationalität und begrenzt opportunistisches Verhalten der Akteure ersetzt. Die unvollständige Information der Akteure führt nunmehr zu Informationsdefiziten. Gleichzeitig bedingt die beschränkte Rationalität der Akteure eine eingeschränkte Verarbeitungsmöglichkeit der vorhandenen Information über die Handlungs-alternativen der Mitakteure. Auch kann der Akteur nicht mehr damit rechnen, dass opportunistisches Verhalten die dominante Strategie seines Gegenübers ist. Nach der Beschränkung der drei Annahmen finden wir einen Akteur vor, der in Entscheidungssituationen von der Komplexität seiner Umwelt überfordert ist. Er handelt in einer Situation der Unsicherheit. Im Folgenden wird erläutert, welche Strategien der Akteur zur Bewältigung der Komplexität verfolgen kann. Ein wichtiges Element dabei stellt der Vertrauensbegriff dar.

\subsubsection{IDENTIFIKATION EINES BRAUCHBAREN VERTRAUENSBEGRIFFS}

Eine allgemeine Definition des Begriffs „Vertrauen“ könnte folgendermaßen lauten: Vertrauen ist eine Reaktion des Akteurs auf Unsicherheiten in seiner Lebenswelt. Um mit dem Begriff Vertrauen arbeiten zu können muss seine Definition jedoch enger gefasst werden. Dazu erscheint es sinnvoll, die Verortung des Vertrauensbegriffs Ripperger ${ }^{150}$ folgend, mit einer Abgrenzung von Begriffen mit ähnlicher Bedeutung zu beginnen.

\subsubsection{Abgrenzung gegen den Begriff „Zuversicht“}

Als Ausgangspunkt hierzu dient uns die Feststellung, dass der Akteur aus einer Situation permanenter Unsicherheit heraus agiert. Er ist mit den allgemeinen Unsicherheiten des Alltags konfrontiert. Um in dieser Situation nicht an ständigen Angstzuständen zu verzweifeln, bedient sich der Akteur eines psychologischen Tricks: Er bildet Erwartungen über das Eintreten kontingenter Ereignisse und kuriert damit seine Informationsdefizite. Durch die Vernachlässigung der Tatsache, dass eine Enttäuschung seiner Erwartungen immer eine prinzipielle Möglichkeit darstellt, gelingt es dem Akteur, die fehlenden Informationen durch Erwartungen zu ersetzen und sich so vom Unsicherheitsdruck zu befreien. Es können nun zwei Modi dieses Vorganges identifiziert werden, die sich dadurch unterscheiden, inwieweit sich der Akteur der möglichen Varianten kontingenter Ereignisse im Klaren ist und inwieweit er seine Erwartungen reflektiert. Werden Erwartungen ad hoc gebildet, so ist sich der Akteur der Unsicherheit nicht be- 
wusst. Seine Reaktion auf die objektive Unsicherheit (Zufall) ist Zuversicht. Vertrauen setzt jedoch voraus, dass der Handlungsträger ein Risiko eingeht. Nur wer sich eines möglichen, also vermeidbaren Schadens bewusst ist, kann etwas riskieren. Das Bewusstsein eines möglichen Schadens entsteht beim Akteur nur, wenn ihm mehrere Handlungsalternativen offen stehen ${ }^{151}$, deren Konsequenzen ihm jedoch nicht vollständig bekannt sind. In Situationen subjektiver Unsicherheit besteht das Risiko eines Irrtums. Eine mögliche Reaktion auf subjektive Unsicherheit ist Vertrauen.

\subsubsection{Abgrenzung gegen den Begriff ,Hoffnung“}

Vertrauen ist jedoch nicht in allen riskanten Situationen eine adäquate Reaktion. Es ist hier eine Unterscheidung nach der Art der kontingenten Ereignisse zu treffen. Exogene Unsicherheit liegt in den Fällen vor, bei denen weder der Akteur noch seine Mitakteure Einfluss auf die Eintrittswahrscheinlichkeit der Ereignisse haben ${ }^{152}$. Die ursächlichen Faktoren der Unsicherheit liegen außerhalb des Systems. ${ }^{153}$ Solchen Umweltunsicherheiten kann der Akteur nur mit Hoffnung begegnen. Ist sich der Akteur jedoch unsicher über die Entscheidungen seiner Interaktionspartner und deren Auswirkungen, so handelt es sich um endogene Unsicherheit. Die Unsicherheit entspringt von innerhalb des Systems und ist ursächlich an den Akteuren festzumachen. Vertrauen kann nicht Einflussfaktoren sondern nur Mitakteuren entgegengebracht werden, denn nur diese handeln und treffen Entscheidungen. Der Akteur vertraut darauf, dass seine Interaktionspartner die Entscheidung treffen, die er von ihnen erwartet.

\subsubsection{Abgrenzung gegen den Begriff „Zutrauen“}

Ob ein Mitakteur die Erwartungen des Vertrauensgebers erfüllt oder nicht, hängt von zwei Faktoren ab: seiner Fähigkeit und seinem Willen. Beide Einflussgrößen sind dem begrenzt rationalen Akteur nicht zugänglich und daher mit Unsicherheit behaftet. Ist der Akteur der Meinung, sein Interaktionspartner ist zur Erfüllung seiner in diesen gesetzten Erwartung fähig, so handelt es sich um Zutrauen. Zutrauen ist die Unterstellung der Fähigkeit und Kompetenz zu einem erwartungskonformen Verhalten. Während sich Zutrauen auf Kompetenzunsicherheit bezieht, ist Vertrauen eine Reaktion auf Verhaltensunsicherheit. Hier besteht Unsicherheit über den Willen des Interaktionspartners, sich gemäß der Erwartungen des Akteurs zu verhalten.

151 vgl. Luhmann 1989, 45

152 Anfang/Urban 1994, 6

153 vgl. Plötner 1995, 21 
Es ergibt sich eine engere Definition des Begriffs Vertrauen: Vertrauen ist eine Reaktion auf die subjektive Unsicherheit über das Verhalten eines bestimmten Interaktionspartners.

\subsubsection{Die MOTIVE HINTER DEM VERTRAUEN}

Bei der Betrachtung des Begriffs Vertrauen bringt der Blick auf die Motive der Vertrauensgeber eine weitere erhellende Differenzierung ${ }^{154}$ : Analog zur Kant'schen Unterscheidung zwischen Legalität und Moralität kann zwischen instrumentellem Vertrauen und maximenbasiertem Vertrauen unter Kooperateuren unterschieden werden.

So wie sich Legalität auf die rein formelle Übereinstimmung des Verhaltens mit den Gesetzen bezieht, bezeichnet instrumentelles Vertrauen die äußerliche Konformität des Verhaltens des Kooperationspartners mit den Kooperationsnormen. Moralität hingegen bezeichnet ein Verhalten, dessen Motivation mit der Moral übereinstimmt. Folglich kennzeichnet maximenbasiertes Vertrauen jenes Vertrauen, hinter dem das Vertrauen selbst als Motiv steht. Während instrumentelles Vertrauen ein Mittel darstellt, einen anderen Zweck zu erfüllen als die Schaffung einer Vertrauenssituation, wird bei maximenbasiertem Vertrauen das Vertrauen selbst als Zweck eingesetzt. Maximenbasiertes Vertrauen ist intrinsisch motiviert. Insofern basiert es darauf, dass der Vertauensgeber die Maxime ${ }^{155}$ internalisiert hat: Du sollst vertrauen.

Nun stellt sich die Frage, aus welchem Grund die Motivation des Vertrauensgebers bei der Entstehung einer Vertrauenssituation relevant ist. Auf den ersten Blick scheint es unerheblich zu sein, warum sich ein Kooperationspartner gemäß der Kooperationsnormen verhält. Es wird aber im Folgenden gezeigt werden, dass sich bestimmte Formen der vertrauensbasierten Komplexitätsbewältigungsstrategien und damit bestimmte Formen von Transaktionsbeziehungen nur dann verwirklichen lassen, wenn das Vertrauen auf Maximen der beteiligten Akteure basiert. Das sind die Fälle, bei denen die Vertrauenswürdigkeit der Interaktionspartner aufgrund fehlender Kontrollmechanismen und der daraus resultierenden Unmöglichkeit von Sanktionen, besonders bedeutsam für das Zustandekommen der Transaktionsbeziehung ist. Zuvor wird, nach den zwei kurzen Exkursen die 
sich der Frage der Funktionsweise von Maximen ${ }^{156}$ sowie der Möglichkeit und Notwendigkeit kooperativen Verhaltens widmen, jedoch noch geklärt, was den Opportunismusspielraum ausmacht, welche Parameter zu seiner Bemessung herangezogen werden und in welchem Verhältnis er zur Unsicherheit der Akteure steht.

\section{Exkurs I: Die Funktionsweise von Maximen}

Dieser Einschub soll verständlich machen, warum Menschen ihrem Handeln Maximen zugrunde legen, obwohl diese Selbstfestlegung oberflächlich betrachtet eine Einschränkung ihrer Freiheit zur Folge hat. Als Erklärungsmodell wird auf das Konstrukt des kategorischen Imperativs abgestellt. Maximen stellen dabei die Bausteine der Moral dar. Wer sich Maximen verpflichtet, handelt in diesem Bereich moralisch. Daher ist die Frage nach der Funktionsweise der Maximen untrennbar mit der Frage nach der Funktionsweise der Moral verknüpft.

Imanuel Kant schrieb als Metapher für sein System: „Der Mensch ist Bürger zweier Welten“. Es besteht zum einen aus der Welt der Erscheinungen, in welcher der Mensch als eben solche miteinbezogen ist. Auf dieser Ebene ist der Mensch in ein Geflecht von Kausalzusammenhängen eingebettet. Sein Willen entspringt den Motiven, den gerichteten Neigungen, die auf der Welt der Erscheinungen basieren, von diesen daher auch abhängig sind. Mit dieser Welt steht der Mensch mit Hilfe seines rezeptorischen Systems in Interaktion. Dieser Vorgang heißt Erfahrung. Der Mensch geht auf dieser Ebene bei der Bildung seines Willens von der Erfahrung aus. Sein Wille ist dadurch von den Erscheinungen und den gegenseitigen Abhängigkeiten, unter denen diese stehen, abhängig. Er entspringt nicht innerhalb der Person, sondern ist von außen bestimmt, fremdbestimmt. Kant nennt diese Form der Willensbestimmung Heteronomie.

Aus der Welt der Erscheinungen kann kein Maßstab abgeleitet werden, der dem Mensch die Beurteilung der "Gutheit" und „Schlechtheit“" ermöglicht, da ein solcher, die Moral, in allgemeiner und notwendiger Weise Geltung haben muss. Wäre dieser Maßstab nicht allgemein gültig, würden die Individuen einzeln nach ihrem persönlichen Maßstab zwischen Gut und Böse unterscheiden. Die Auffassung des Einen lässt aber den Anderen nicht unberührt. Es kommt zu einem Konflikt. Das heißt, der Maßstab muss Allgemeingültigkeit beanspruchen. Die Erfahrung ist aber von dem Verhältnis des Individuums zu den Erscheinungen ab1990,13 
hängig, also subjektiv, und kann daher nicht Basis für einen allgemein gültigen Maßstab sein. Die Moral muss auf einer anderen Basis stehen.

Kant findet diese Basis: die Vernunft. Konkreter: die praktische Vernunft, weil diese genauso wie die Moral auf das Handeln, dem Ausdruck des Willens, abzielt. In der Vernunft, der zweiten Ebene des Systems, ist der Prozess der Willensbildung frei von Momenten, die außerhalb des Individuums liegen. Der Wille entspringt also der reinen Vernunft. Diese Form der Willensbildung nennt Kant Autonomie, im Sinne von Unabhängigkeit von allem äußeren Einfluss. Diese Unabhängigkeit begründet auch die Freiheit des Menschen. Es ist wichtig festzustellen, dass Kant mit Freiheit gerade nicht meint, dass der Mensch tun und lassen kann was er will, sondern ganz im Gegenteil, nur eine einzige Weise zu handeln hat. Nämlich gemäß der Pflicht. Diese ist aber ein Zwang, der nicht von außen auf das Individuum einwirkt, sondern aus der Vernunft selbst entspringt. Ist das Individuum vernünftig, folgt es also der Vernunft, und Kant geht für den Menschen davon aus, hat es gar keine andere Wahl, als der Pflicht Folge zu leisten, da es sich sonst nicht nur gegen die Pflicht entscheidet, sondern sich selber auch aus dem Kreise der Vernünftigen ausschließt. Das ist nach Kant nicht möglich. Wie kommt es aber zu dieser Pflicht? Und wie kann der Mensch erkennen, was im konkreten Fall die Pflicht ist?

Die erste Frage führt uns zum Konstrukt des kategorischen Imperativs. Es ist an dieser Stelle angebracht, kurz den Unterschied zwischen kategorischem und hypothetischem Imperativ aufzuzeigen. Jeder Imperativ drückt ein Sollen aus. Der Unterschied besteht nicht in der hypothetischen oder kategorischen Formulierung, sondern in der Art und Weise wie er gebietet. Im Falle des hypothetischen Imperativs lässt sich das Mittel logisch aus dem Zweck ableiten. Wenn der Zweck darin besteht ein Bier zu trinken, und es gibt nur das Mittel eines zu kaufen, so lässt sich das Mittel logisch aus dem Zweck ableiten, nämlich man kauft ein Bier. Der Imperativ ist also am Zweck festgemacht. Damit ist die Nötigung vom Zweck abhängig und damit vom Subjekt, welches den Zweck verfolgt. Ein hypothetischer Imperativ kann somit nicht Allgemeingültigkeit beanspruchen und kann dadurch auch nicht Grundlage der Moral sein. Der kategorische Imperativ hingegen betrifft nur die Form und das Prinzip der Handlung. Das Gute der Handlung besteht in der Gesinnung und nicht im Resultat, dem Zweck. Der kategorische Imperativ ist dem Prinzip der Moralität verpflichtet. Es kommt nicht auf die äußere Übereinstimmung der gesetzten Handlungen mit den Gesetzen an (Legitimität), sondern die gute Gesinnung, also die innere Identität des Willens mit den moralischen Gesetzen ist wesentlich (Moralität). 
Wie oben gezeigt, kann nur eine Regel, die der Vernunft entspringt, Allgemeingültigkeit beanspruchen. Eine Regel mit einem Subjekt und einem Objekt ist aber von der Welt der Erscheinungen (Ebene1) nicht unabhängig, da sowohl Objekt als auch Subjekt in dies eingebunden sind. Die Forderung nach Unabhängigkeit, also nach Autonomie, erfüllt nur eine Regel, die sowohl vom Subjekt als auch vom Objekt abstrahiert. Eine solche Regel ist eine formale. Damit ist die Forderung nach Allgemeingültigkeit erfüllt. Wie kommt man aber zur Notwendigkeit der Gültigkeit? Kant weist den Menschen dazu an, seine eigenen Handlungsrichtlinien, seine Maximen, zur Probe an dem kategorischen Imperativ zu messen. Ist also die Maxime seines Handelns so, dass er wünschen könnte, dass sie ein allgemeingültiges Gesetz werden solle, dann entspricht sie der formalen Vorgabe des kategorischen Imperativs und die Handlung, die ihr entspringt, ist moralisch. Der Mensch als vernünftiges Individuum muss also jede seiner Maximen darauf testen, ob sie diesem Anspruch genügt. Der Zwang, der daraus entspringt, heißt nach Kant Pflicht. Der Mensch ist also moralisch genötigt, das Gute zu tun.

Diese Pflicht steht nicht im Widerspruch zur Freiheit. Der Mensch ist frei von den Zwängen der Welt der Erscheinungen, wenn er autonom handelt, er muss aber aufgrund seiner Vernünftigkeit gemäß der Pflicht handeln.

Kant kommt zu dem Ergebnis, dass Moral, gedacht als Handeln nach Maximen, nur durch die Freiheit des Willens möglich ist. Ausgangspunkt ist die Vorstellung eines leeren Willens. Er besteht rein aus dem formalen Wollen. Der Wille verpflichtet sich nun auf den Inhalt, den sich die Vernunft selbst (autonom) gegeben hat. Der notwendige Willensinhalt muss also, da er von der Vernunft vorgegeben ist, mit einem Willen gleichgerichtet sein, der ja selbst auf die Vernunft verpflichtet ist. Der Widerspruch in der Forderung nach einem freien Willen mit notwendigem (zwingenden) Inhalt wird mit Hilfe des Hilfskonstruktes des leeren Willens eliminiert. Damit wird auch klar, was die Voraussetzung autonomen Handelns ist, nämlich die Möglichkeit seinem Willen einen Inhalt zu geben, der nicht der Befriedigung unserer Wünsche und Bedürfnisse dient, sondern diesen sogar entgegengerichtet ist. Der Wille ist frei von jeder Abhängigkeit, da sein Inhalt keinen Bedingungen unterworfen ist. Die moralischen Gesetze sind so auf eine objektive Basis gestellt. In diesem Konstrukt, in dem der Wille einen notwendigen Inhalt durch die Verpflichtung auf die Vernunft hat, fallen wollen und sollen zusammen. Der Mensch kann nur das wollen, was er auch soll - nämlich Handeln auf der Basis seiner Maximen - weil er ein vernünftiges Individuum ist. 
Damit ist auch klar, dass alle Einwände gegen die praktische Relevanz maximenbasierten Vertrauens, die darauf abstellen, dass die Akteure im Wirtschaftsleben auf Grund äußerer Umstände nicht gemäß ihrer Maximen handeln können, weil sie sonst im Wettbewerb unterliegen ${ }^{157}$, ins Leere gehen. Denn die vorgebrachten Sachzwänge werden als Denkzwänge entlarvt. Damit verlagert sich die Frage nach der praktischen Relevanz maximenbasierten Vertrauens von „Warum kann der Akteur maximenbasiert vertrauen?“ auf „Warum soll der Akteur maximenbasiert vertrauen?" um. Da es in vorliegender Arbeit um kooperatives Handeln auf der Basis von Vertrauen geht, kann die Frage enger gefasst werden: „Warum soll der Akteur kooperativ handeln?" Dieser Frage widmet sich Exkurs II.

\section{Exkurs II: Die Möglichkeit moralischer Handlungen im Markt - Warum das Wirtschaftssubjekt kooperativ handeln „soll“6}

In diesem Exkurs soll geklärt werden, auf welcher Basis kooperatives Verhalten für die Wirtschaftssubjekte eine gebotene Handlungsalternative darstellt und wie der normative Anspruch dieser deontischen Aussage begründet werden kann. Er ist die Antwort auf Einwände, wie sie hier beispielhaft in Form der Argumentation von Lenz dargestellt wird:

„Streng rationale Begründungsversuche zur Entstehung von Normen führen nun allerdings nach meinem Dafürhalten in eine Paradoxie. Normen sollen ja die Menge möglicher Handlungsweisen beschränken, bestimmte davon als geboten, andere als verboten ausgezeichnet. Sie schreiben dem Adressaten der Norm ein bestimmtes Handeln vor, sind somit präskriptive Aussagen. Normen sind offensichtlich dann nicht erforderlich, wenn es keine Anreize gibt, sich nicht an sie zu halten, sie zu übertreten. Bei allen Begründungsversuchen, die zu zeigen versuchen, dass die Etablierung und Einhaltung einer Norm im wohlverstandenen langfristigen Eigeninteresse liegt, wird gar nicht mehr verständlich, weshalb eigentlich eine verhaltensbeschränkende Norm (etwa ,Du sollst dich nicht unkooperativ verhalten!') erforderlich ist. Wenn die Rücksichtnahme auf andere im langfristigen Eigeninteresse liegt, dann handeln diejenigen, die sich nicht an die Norm halten, ja offensichtlich gegen ihre eigenen langfristigen Interessen, somit irrational. Da diese Begründungsversuche aber auf rational handelnden Individuen aufbauen, kann es normabweichendes Verhalten eigentlich gar nicht geben. Die zu begründen versuchte Norm erweist sich angesichts kühl und langfristig kalkulierender Individuen als überflüssig. Gesteht man jedoch die Existenz normabweichenden Verhaltens zu, sind Normen auf diese Weise nicht mehr begründungsfähig." 158

Da eine Vielzahl der Ökonomen jedoch der Meinung ist, die Purifizierung der Ökonomie nach dem Vorbild der Naturwissenschaften und die Schaffung einer wertfreien Ökonomie, die frei von jeglichen normativen Momenten ist, sei gelungen, erscheint es notwendig, zuvor den mit Hilfe des Sachzwangarguments

157 Als deutliches Beispiel dieses Kategorienfehlers sei die Arbeit von A. Shleifer genannt: Shleifer 2004

158 Lenz 1990, 21 
verschleierten Regressstopp der Ökonomen, aufbauend auf den Ausführungen in Exkurs I, zu entlarven, um so die Möglichkeit moralischen Handelns im Wirtschaftsleben noch deutlicher aufzuzeigen. ${ }^{159}$

Die normativen Postulate der Ökonomen erscheinen so lange nicht als begründungsbedürftig, so lange behauptet wird, dass die Wirtschaftssubjekte durch Sachzwänge an diese Postulate gebunden sind. So ersetzt ein naturgesetzliches „Müssen“ ein argumentierbares „Sollen“. Die Postulate der Ökonomen gebieten den Wirtschaftssubjekten zwar ein bestimmtes Verhalten (normative Aussage), entziehen sich aber mit dem Verweis auf die angeblich empirisch im Wirtschaftsleben vorfindbaren Sachzwänge ihrer Begründungsverantwortung. ${ }^{160}$

Selbst wenn die behaupteten Sachzwänge die Wirtschaftssubjekte auf das gebotene Verhalten festlegen würden, wäre ein solcher Legitimationsversuch der normativen Aussagen der Ökonomie nicht ohne naturalistischen Fehlschluss möglich! ${ }^{161}$ Tatsächlich handelt es sich bei den behaupteten Sachzwängen aber vielmehr um Denkzwänge ${ }^{162}$, denn Sachzwänge herrschen nur dort, wo Naturgesetze einen Ursache-Wirkungs-Zusammenhang determinieren. ${ }^{163}$ Wirtschaften als soziale Handlung kann somit niemals durch Sachzwänge bestimmt sein. Ulrich schließt:

„Auch das strikteste erfolgsrationale Handeln bleibt jedoch, solange es das Handeln eines menschlichen Subjekt ist, in Wirklichkeit stets vom subjektiven Willen zum entsprechenden Erfolg, also von einer im Prinzip freien Zweckwahl abhängig und ist eben deshalb argumentationszugänglich. [...] Daraus ergibt sich, dass der ökonomische Determinismus als empirische Hypothese überhaupt keinen Sinn macht. Zur Klärung der Frage, ob und wieweit die Wirtschaftssubjekte im Markt sich denn nun zu Recht oder zu Unrecht auf Sachzwänge berufen, die sie (angeblich) daran hindern, moralischen Ansprüchen zu entsprechen, vermag solches Sachzwangdenken rein gar nichts zu besagen. Wer auf seiner Basis gegen die Möglichkeit moralischen Handelns unter marktwirtschaftlichen Bedingungen argumentiert, der konfundiert offenbar den rein modelltheoretischen Charakter des ökonomischen Determinismus ,methodisch' mit der Realität. “164

159 „Es gilt dabei genau aufzuzeigen, wo und mit welchen Methoden sich die immer schon normative Ökonomik ihre als solche nicht begründete normative Kraft erschleicht, um sich als (die bessere) ,Wirtschaftsethik ohne Moral' präsentieren zu können.“ (Ulrich 1997, 117)

160 vgl. beispielsweise Schleifer (2004)

161, ,Man kann das System nicht normativ in Frage stellen, indem man lediglich die innerhalb dieses Systems zustande gekommenen moralischen Urteile auf ihre sozioökonomischen Bedingungen hinterfragt." (Tugendhat 1993, 16)

162/162 Ulirch 1997, 131

163 I. Kant unterscheidet analog zwischen Denkzwängen „Kausalität durch Freiheit“ (Gründe) und Sachzwängen „Kausalität nach Gesetzen der Natur“ (Ursachen). vgl. Kant 1981, 426

164 Ulrich 1997, 153 
Bisher wurde gezeigt, dass die Handlungen der Wirtschaftssubjekte nicht durch Sachzwänge determiniert, sondern vom Willen der Wirtschaftssubjekte abhängig sind. Somit konnte die Möglichkeit der Wirtschaftssubjekte aufgezeigt werden, ihren Handlungen moralische Prinzipien zugrunde zu legen. Auf dieser Einsicht aufbauend, können nun die zwei eigentlichen Fragen geklärt werden: 1. Wie kann kooperatives Verhalten als normatives Prinzip etabliert werden, so dass es für die Wirtschaftssubjekte eine gebotene Handlungs-alternative darstellt? (Zwecksetzungsproblematik) 2. Inwieweit ist es den Wirtschaftssubjekten zumutbar, sich kooperativ zu verhalten? (Zumutbarkeitsproblematik)

Zwecksetzungsproblematik und Zumutbarkeitsproblematik greifen ineinander, denn es geht allgemein gesprochen darum, zu ergründen, welche Zwecksetzung ${ }^{165}$ dem Wirtschaftssubjekt zumutbar ist.

Das heißt: Das Wirtschaftssubjekt soll sich bei seiner Zweckwahl nach moralischen Prinzipien richten, soweit ihm die aus seinen Handlungen entspringenden Konsequenzen zumutbar sind. Somit kann jeder Zweck im Zuge eines Zwecksetzungsdiskurses als normatives Prinzip formuliert werden, dessen Konsequenzen dem Wirtschaftssubjekt, dessen Handlungen es normiert, zugemutet werden können.

„Das entscheidende ethische Kriterium für die Lösung so verstandener Zumutbarkeits- bzw. Zwecksetzungsprobleme kann - wie stets bei normativen Problemen - nur die argumentative Universalisierbarkeit der Geltungsansprüche im Sinne der intersubjektiven Austauschbarkeit der Perspektiven zwischen den Akteuren und allen Betroffenen sein."“166

Um auf die eingangs formulierte Frage: Warum soll sich ein Wirtschaftssubjekt kooperativ verhalten? Antwort zu geben, muss geprüft werden, ob kooperatives Verhalten als normatives Prinzip dem Wirtschaftssubjekt zumutbar ist. Diese Prüfung hat, wie oben gezeigt, im Zumutbarkeitsdiskurs stattzufinden, und kann nicht hier entschieden werden. Das bedeutet, dass weder an dieser Stelle in diesem Text, noch an irgendeiner anderen Stelle in einem anderen Text, eine allgemeingültige Begründung für die Notwendigkeit moralischen Handelns im Markt ge-

165 „Der Zweckbegriff bezeichnet diejenige Wirkung bzw. den Komplex von Wirkungen, die das Handeln rechtfertigen sollen, also stets nur einen Ausschnitt aus dem Gesamtkomplex der Wirkungen. Sein ,Thema' ist nicht die Bewirkung jener ausgezeichneten Wirkungen, sondern das Verhältnis ihres Wertes zu den Werten der Nebenwirkungen (einschließlich der Wirkungen anderer Möglichkeiten des Handelns, auf die man bei einem bestimmteren Engagement verzichten muss). Die Zwecksetzung besagt, dass der Wert der bezweckten Wirkungen ungeachtet der Werte oder Unwerte der Nebenwirkungen bzw. der aufgegebenen Wirkungen anderer Handlungen das Handeln zu begründen vermag." (Luhmann 1977, 44)

166 Ulrich 1997, 159 und die dort zitierte Literatur 
geben werden kann, da über die Zumutbarkeit einer gebotenen Handlung nicht pauschal - von der konkreten Situation abstrahiert - entschieden werden kann. Eine solche allgemeine Feststellung der Notwendigkeit der Gültigkeit einer Maxime, wäre selbst zu legitimieren - was zwangsläufig in einem infiniten Regress endete - und würde den Zwecksetzungsdiskurs, der ja gerade die Legitimierung der Maxime leisten soll, übergehen. Dennoch erhebt diese Arbeit sehr wohl den Anspruch, dem Zwecksetzungs- bzw. Zumutbarkeitsdiskurs Argumente bereit zu stellen, die seinen Ausgang wesentlich beeinflussen können.

Bezüglich des Ergebnisses des Zumutbarkeitsdiskurses im Bereich der langfristigen, komplexen Transaktionsbeziehungen kann man optimistisch sein, denn wie diese Arbeit zu zeigen versucht, sind die Konsequenzen aus kooperativen Verhalten für den Handlungsträger weitgehend positiv und daher zumutbar, auch wenn das dem konkret Handelnden nicht vorab bewusst ist. Diese Vermutung wird von den Ergebnissen der Simulation von Axelrod gestützt. ${ }^{167}$ Denn wenn später eintretende positive Konsequenzen kooperativen Verhaltens dem Akteur (auf Grund mangelnder Erfahrung) nicht selbst bewusst sind, führt der Zumutbarkeitsdiskurs über die Erschließung des gesellschaftlichen Wissensvorrates zu deren Einbeziehung bei der Beurteilung der Zumutbarkeit. Auf diese Weise gewährleistet der Zumutbarkeitsdiskurs eine mittel- bis langfristige Perspektive bei der Entscheidung über die Zumutbarkeit einer Zwecksetzung für den Akteur.

Zusammenfassend kann festgestellt werden: Moralisches Handeln ist im Wirtschaftsleben entgegen der Behauptungen vieler Ökonomen möglich, da die Spielregeln des Marktes keine Naturgesetze sind und daher keine Sachzwänge ausüben können. Sie sind vielmehr prinzipiell argumentierbare Denkzwänge, die durch die freie Zwecksetzung der Wirtschafssubjekte durchbrochen werden können. Die Basis für die Formulierung deontischer Aussagen stellt der öffentliche Diskurs dar. Er bestimmt im Allgemeinen, welchen Handlungsprinzipien normative Aussagekraft zugesprochen wird (Zwecksetzungsdiskurs) und im Speziellen, ob die Befolgung dieser moralischen Prinzipien für das Wirtschaftssubjekt in einer konkreten Situation zumutbar ist (Zumutbarkeitsdiskurs). Im Falle der Maxime: Du sollst dich kooperativ verhalten!, kann für den Bereich der langfristigen, komplexen Transaktionsbeziehungen davon ausgegangen werden, dass im Diskurs sowohl die allgemeine, als auch die spezielle Frage positiv beantwortet wird. Damit stellt kooperatives Verhalten bei langfristigen, komplexen Transaktionsbeziehungen eine mögliche Maxime dar. Thre Befolgung ist, betrachtet man die überwiegend positiven Konsequenzen über die mittlere und lange Frist, in kon-

167 vgl. Axelrod, R. (1986): The Evolution of Cooperation. Basic Books, New York. 
kreten Situationen mit hoher Wahrscheinlichkeit zumutbar. ${ }^{168}$ Daraus ergibt sich: Das Wirtschaftssubjekt soll sich bei langfristigen, komplexen Transaktionsbeziehungen kooperativ verhalten, weil ein solches Verhalten zumutbar ist.

\subsection{Vom Vertrauen zur Selbstverpflichtung}

\subsubsection{DER OPPORTUNISMUSSPIELRAUM}

Ist der unvollständig informierte und begrenzt rationale Akteur an einer Kooperation beteiligt, so ist er mit dem Problem konfrontiert, nicht alle Informationen über seine Kooperationspartner zu besitzen und zu begreifen. In kooperativen Transaktionsbeziehungen besteht in jeder Phase die prinzipielle Möglichkeit, dass ein Kooperateur den gemeinsamen langfristigen Vorteil zugunsten eines defektiven Verhaltens aufgibt, um den kurzfristigen Vorteil zur Gänze selbst abzuschöpfen ${ }^{169}$. Die Option, sich seinem Geschäftspartner gegenüber unfair zu verhalten und das versprochene Verhalten nicht zu verwirklichen, begründet für jeden Kooperationspartner einen Opportunitätsspielraum ${ }^{170}$. Zwei Faktoren beeinflussen die Größe der Unsicherheit des Akteurs über das Verhalten seines Kooperationspartners. Einerseits ist die Größe des Opportunitätsspielraums proportional zum potentiellen Vorteil aus unfairem Verhalten. Andererseits ist die Unsicherheit über das Verhalten des Kooperationspartners abhängig von dessen Opportunismusneigung, die die Wahrscheinlichkeit der Ausnutzung des vorhandenen Opportunismusspielraums bezeichnet.

Je größer der Opportunismusspielraum des Kooperationspartners ist, desto weniger kann der Akteur die Komplexität seiner Umwelt durch Bildung von Erwartungen reduzieren, da die Vernachlässigung der Möglichkeit einer Enttäuschung seiner Erwartung als immer weniger adäquat erscheint. Daher muss es das Ziel jedes Kooperateurs sein, möglichst sicherzustellen, dass sich der Interaktionspartner in der vereinbarten und daher erwarteten Weise verhält. Für dieses Unterfangen stehen zwei Ansatzpunkte zur Verfügung: Der Akteur kann die Payoffmatrix der Art verändern, dass sich der Vorteil aus opportunistischem Verhalten reduziert und damit unfaires Verhalten für den Transaktionspartner unattraktiver

168 Auch wenn es in Situationen nicht zumutbar ist, kooperativ zu handeln, besteht noch immer das moralische Gebot: Du sollst Dich kooperativ verhalten! Ein Verhalten, das von dieser Maxime abweicht ist dennoch eine Willensentscheidung und nicht durch irgend einen Sachzwang determiniert.

169 vgl. Wurche 1991, 147

170 vgl. Muris 1981, 521; John 1984, 288; Provan/Skinner 1989, 203; Hauser 1991, 112; Poppo 1991, 20; Dahlstrohm/Boyle 1994, 53; Noorderhaven 1995, 8; Gassenheimer/Baucus/Baucus 1996, 68: Wathne/Heide 2000 
wird. Damit reduziert er den Opportunitätsspielraum seines Interaktionspartners, gleichzeitig aber auch seine Unsicherheit über dessen Verhalten. Die zweite Möglichkeit besteht in der Reduzierung der Opportunitätsneigung des Kooperationspartners.

Im Folgenden werden zuerst knapp zwei Strategien zur Reduzierung des Opportunismusspielraums besprochen: ausgleichende Investitionen und Schutz durch Verträge. Danach wird auf Selbstverpflichtung als Strategie zur Reduzierung der Opportunismusneigung näher eingegangen. Es wird gezeigt werden, wie maximenbasierte Vertrauensverhältnisse entstehen und warum sie eine Grundlage für die Entstehung und den Bestand von Synergiesystemen bieten. Zuvor wird der Gang der Argumentation als Orientierungshilfe für den Leser in Abbildung 3 schematisch dargestellt.

\subsubsection{BEGRENZUNG DES OPPORTUNISMUSSPIELRAUMS ALS KOMPLEXITÄTS- REDUKTIONSSTRATEGIE}

Das Ziel jeder Komplexitätsreduktionsstrategie durch Begrenzung des Opportunismusspielraums des Kooperationspartners ist, die Anzahl der Situationen zu reduzieren, in denen ein potentieller Vorteil aus opportunistischem Verhalten für den Interaktionspartner existiert. Genau dies kann der Aufbau instrumentellen Vertrauens zwischen den Kooperateuren leisten, denn das instrumentelle Vertrauen bringt den Kooperateur dazu, sich gemäß der Kooperationsnormen zu verhalten. Es ist für die Reduzierung des Opportunismusspielraumes irrelevant, wie sein normenkonformes Verhalten motiviert ist. ${ }^{171}$ In dem Ausmaß, in dem der Opportunismusspielraum eines Akteurs reduziert wird, sinkt die Unsicherheit seines Interaktionspartners über das Verhalten dieses Akteurs und die Komplexität der Transaktionsbeziehung wird reduziert.

Die Reduktion der Komplexität der Kooperationsbeziehung durch Begrenzung des Opportunismusspielraums auf der Basis instrumentalen Vertrauens ist einerseits durch detaillierte Verträge und andererseits durch ausgleichende Investitionen operationalisierbar ${ }^{172}$.

171 Es kommt auf die „Legalität“ der Handlung, also auf die äußere Übereinstimmung der Handlung mit der Norm an.

$172 \mathrm{vgl}$. Gierl 2001, 59 
Abb. 3: Gang der Argumentation ${ }^{173}$

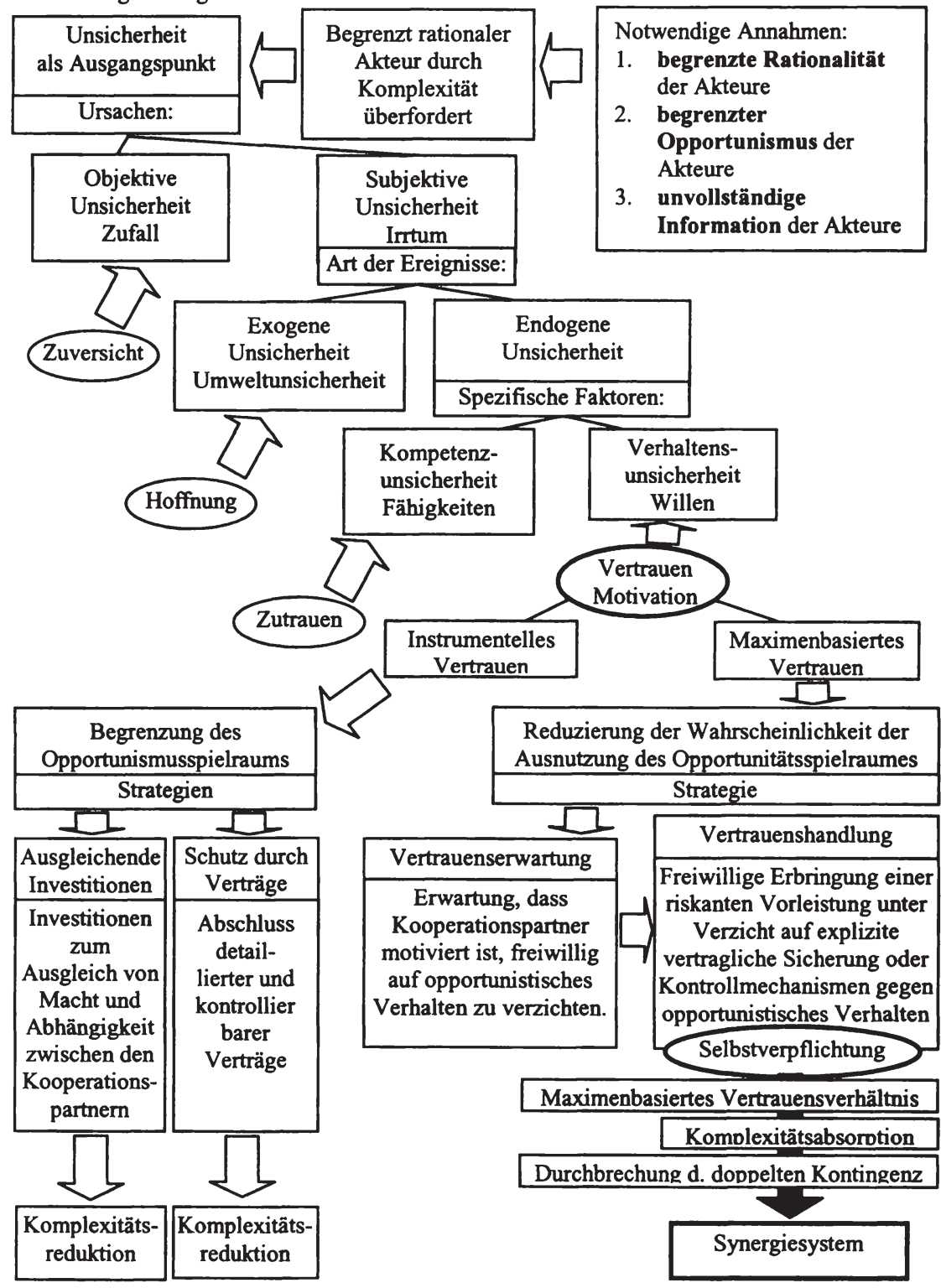

173 Quelle: Eigene Darstellung in Anlehnung an: Rössl 1996, $321 \mathrm{ff}$, Ripperger 1998, 34ff; Osterloh 2000, 103; Gierl 2001, 59 sowie Kettel 2002, 4. 
Letztere Strategie hat ihre theoretischen Wurzeln in der Power-DependenceTheory. Es wird argumentiert, dass es durch eine unterschiedliche Interessensintensität an der Kooperation in kooperativen Transaktionsbeziehungen zu einem Machtungleichgewicht kommt. Der Partner, der weniger Interesse an dem Bestand der Kooperation hat, besitzt im Ausmaß der Differenz der Interessensintensitäten der Kooperateure Macht über seinen Kooperationspartner. ${ }^{174}$ Der Schwächere ist gezwungen, mehr für den Bestand der Kooperation zu investieren ${ }^{175}$. Sowohl die Machtposition als auch seine geringeren spezifischen Investitionen vergrößern den Opportunitätsspielraum des weniger am Bestand der Kooperation Interessierten.

Um den so entstandenen Opportunismusspielraum zu begrenzen, können ausgleichende Investitionen getätigt werden. ${ }^{176}$ Diese zielen auf eine Angleichung der Interessensintensität der Kooperationspartner am Bestand der Transaktionsbeziehung ab. Mögliche Formen ausgleichender Investitionen sind Sprungwerbung und finanzielle Beteiligungen am Unternehmen des mächtigeren Kooperationspartners ${ }^{177}$. Durch diese Maßnahmen steigt das Kooperationsinteresse des bislang mächtigeren Kooperateurs an und reduziert dessen Opportunismusspielraum. Dies wiederum führt zu einer Reduktion der Komplexität der Transaktionsbeziehung.

Theoretisch denkbar wären auch ausgleichende Investitionen, die auf eine Angleichung der von den Kooperationspartnern getätigten kooperationsspezifischen Investitionen abzielten. Jedoch stellt sich die Frage, mit welchen Mitteln der mächtigere Kooperateur motiviert werden soll, Investitionen zu tätigen, die den Zweck haben, seine Machtbasis zu untergraben. Als dementsprechend gering erscheint daher die praktische Relevanz dieser Form ausgleichender Investitionen.

Die zweite Grundstrategie zur Reduzierung des Opportunismusspielraums des Kooperationspartners auf der Basis instrumentellen Vertrauens ist der Abschluss detaillierter Verträge unter den Kooperateuren ${ }^{178}$. Solche Verträge listen penibel die einzelnen Pflichten und Rechte der Vertragspartner, Kontrollmaßnahmen über deren Einhaltung und Sanktionsmaßnahmen bei Vertragsverletzungen auf. Durch möglichst vollständige Erfassung aller Details und Eventualitäten der Transak-

174 vgl. Engel 1999, 8

175 vgl. Rößl 1994, 243

176 vgl. Heide/John 1988

177 vgl. Gierl 2001, 59

178 vgl. Heide/John 1988, 22; Heide/John 1992, 35 sowie Heide 1994, 73 
tionsbeziehung durch die vertraglichen Regelungen wird erreicht, dass opportunistisches Verhalten erkannt wird und die Folgen solchen defektiven Verhaltens berechenbar sind. Die Möglichkeit der Kontrolle der Einhaltung der Kooperationsnormen verringert die Wahrscheinlichkeit, mit der defektives Verhalten vom Kooperationspartner unbemerkt bleibt und eliminiert so alle Handlungsoptionen, die nur durch einen unaufgedeckten Verstoß gegen die Kooperationsnormen einen Vorteil für den aktiven Handlungsträger generiert hätten. Die Situationen, in denen für einen Kooperateur durch opportunistisches Verhalten ein potentieller Vorteil besteht, reduzieren sich, sein Opportunismusspielraum wird beschränkt. Dies und die Absehbarkeit der Folgen defektiven Verhaltens führen zu einer Reduktion der Komplexität der Transaktionsbeziehung.

Für den Abschluss detaillierter Verträge müssen jedoch einige Bedingungen erfüllt sein, wodurch die Anwendbarkeit dieser Komplexitätsreduktionsstrategie erheblich eingeschränkt wird. Erstens muss der Kooperationspartner bereit sein, einen solchen Vertrag abzuschließen. Außerdem haben bei Vertragsabschluß alle Rechte und Pflichten der Kooperateure festzustehen. Dazu ist zumindest detailliertes Wissen über das gewünschte Ergebnis der Kooperation nötig. Diese Informationen stehen bei komplexen Transaktionsbeziehungen jedoch nicht zur Verfügung. Auch ist zu erwarten, dass mit steigendem Zeithorizont der Transaktionsbeziehung die Bereitschaft der Kooperationspartner sinkt, detaillierte Verträge abzuschließen und sich auf lange Frist genau festgeschriebene Pflichten aufzuerlegen. Es ist daher auch der Bereich der hochgradig unsicheren, langfristigen, komplexen Transaktionsbeziehungen, in dem die Beschränkung des Opportunismusspielraums als Strategie zur Komplexitätsbewältigung scheitert. Bei Transaktionsbeziehungen, die durch hohe Komplexität und eine langfristige Perspektive gekennzeichnet sind, ist der Aufbau maximenbasierten Vertrauens notwendig, um über die Begrenzung der Opportunismusneigung der Kooperateure eine Komplexitätsabsorption zu erreichen.

\subsubsection{BEGRENZUNG DER OPPORTUNISMUSNEIGUNG ALS KOMPLEXITÄTS- ABSORPTIONSSTRATEGIE}

Die zweite grundsätzliche Möglichkeit zur Reduktion der Verhaltensunsicherheit besteht für den Akteur in der Begrenzung der Opportunismusneigung seines Interaktionspartners. Durch eine Senkung der Wahrscheinlichkeit, mit der sich der Interaktionspartner opportunistisch verhält, wird die Erwartung eines Verhaltens, das konform mit den Kooperationsnormen geht, als Modell der Zukunft für den Akteur adäquater, da die Vernachlässigung der Möglichkeit einer Enttäuschung seiner Erwartung weniger riskant ist. Dadurch ist die Unsicherheit des Akteurs 
über das Verhalten seines Interaktionspartners reduziert, was wiederum eine Verringerung der Komplexität der Transaktionsbeziehung zur Folge hat.

Strategien, die darauf abzielen die Opportunismusneigung eines Kooperationspartners zu begrenzen, müssen derart Einfluss auf dessen Motivationsstruktur nehmen, dass ihm das Einhalten der Kooperationsnormen wertvoller erscheint, als potentielle Vorteile defektiven Verhaltens. Genau das kann der Aufbau von maximenbasiertem Vertrauen leisten. Durch den Aufbau maximenbasierten Vertrauens verändert sich die Wertehaltung der Kooperationspartner und sie übernehmen den normativen Satz „Du sollst vertrauen“ als Maxime. ${ }^{179}$ Das führt dazu, dass der Aufbau und der Bestand einer Vertrauensbeziehung von den Kooperationspartnern selbst als Wert ${ }^{180}$ empfunden wird. Defektives Verhalten fürt zum Verlust dieses Wertes und erscheint daher für die Beteiligten einer maximenbasierten Vertrauensbeziehung als weniger attraktiv. Die Opportunismusneigung der Transaktionspartner ist begrenzt.

Ist Komplexitätsbewältigung auf der Basis instrumentellen Vertrauens nicht möglich oder von den Kooperationspartnern unerwünscht ${ }^{181}$, so rückt die Vertrauenswürdigkeit als auslösendes Moment des Entstehungsprozesses maximenbasierter Vertrauensverhältnisse in das Zentrum der Aufmerksamkeit. Wie sehr ein Akteur seinen Interaktionspartner als würdig erachtet, Vertrauen entgegengebracht zu bekommen, hängt primär von den Motiven ab, die hinter den Handlungen des Interaktionspartners stehen. Dabei wird intrinsisch motivierte Konformität des Handelns mit den Kooperationsnormen als wertvoller eingeschätzt als extrinsisch motivierte Erfüllung der Erwartungen des Kooperationspartners. ${ }^{182}$ Die Ursache der höheren Wertschätzung des maximenbasierten Vertrauens ist dessen höhere Stabilität über die Zeit. ${ }^{183}$ So könnte sich der vom Interaktionspartner verfolgte Zweck, dem instrumentelles Vertrauen als Mittel dient, im Zeitablauf ändern und ein anderes Mittel, z.B. defektives Verhalten, könnte dem Interaktionspartner als für diesen neuen Zweck dienlicher erscheinen. Mit dem Zweck ändern sich die Mittel. Maximenbasiertes Vertrauen ist hingegen gerade dadurch gekennzeichnet, dass es nicht Mittel zum Zweck, sondern Selbstzweck ist. Da-

179 Das geschieht dann, wenn sie wünschen könnten, die Maxime „Du sollst vertrauen“ sollte ein allgemeingültiges Gesetz werden. (Probe am kategorischen Imperativ)

180 ,Economists tend to think of value in exchange as something that exists independent of transaction" (Berger/Noorderhaven 1997, 2) but "the value of the relationship itself is typically ignored and the interpersonality of the transaction is assumed." (Murakami/Rohlen 1992, 70)

$181 \mathrm{vgl}$. Engel 1999, 48

182 vgl. Osterloh/Weibel 2000, 104; Frank 1988 sowie Ripperger 1998

183 vgl. Noorderhaven 1996, 110 
durch, dass im Falle maximenbasierten Vertrauens das Vertrauen selbst als Zweck eingesetzt ist, ergibt sich eine Widerstandsfähigkeit dieser Form des Vertrauens gegenüber Veränderungen im Zeitablauf.

\subsection{Das maximenbasierte Vertrauensverhältnis}

Glaubwürdig vermitteltes maximenbasiertes Vertrauen (Vertrauenserwartung und -handlung von A) ist damit die einzige Basis, die dem Akteur genügend Vertrauenswürdigkeit verleiht, seinen Interaktionspartner dazu anzuregen, von ihm einen freiwilligen Verzicht auf opportunistische Handlungen zu erwarten (Vertrauenserwartung von $\mathrm{B}$ ) und auf der Basis dieser Erwartung seinerseits eine riskante Vorleistung (Vertrauenshandlung von B) zu erbringen. Das initiale Moment des Entstehungsprozesses maximenbasierten Vertrauens ist die Vertrauenswürdigkeit der Kooperationspartner, die davon abhängt, inwieweit sie sich der Maxime „Du sollst vertrauen ", verpflichtet fühlen. Diese Selbstverpflichtung ist für den Interaktionspartner nur durch die Vertrauenshandlung erlebbar. Vertrauenshandlungen sind Akte, die dem Kooperationspartner demonstrieren, dass man sich ihm auf Grund des Vertrauens, das man in ihn setzt, schutzlos ausliefert. ${ }^{184}$ Sie basieren auf der Vertrauenserwartung: der Erwartung, dass der Kooperationspartner vertrauenswürdig ist und freiwillig auf opportunistisches Verhalten verzichtet. ${ }^{185}$ Die Vertrauenshandlung als erlebbarer Ausfluss des Willens des Interaktionspartners wird als Indiz für dessen Motivation herangezogen und bildet die Grundlage der eigenen Vertrauenserwartung des Akteurs, die zu einer eigenen Vertrauenshandlung führt.

Bei der Entstehung maximenbasierten Vertrauens handelt es sich um einen Prozess, bei dem sich unter den Kooperationspartnern auf der Basis der gegenseitig zugestandenen Vertrauenswürdigkeit parallel aufeinander gerichtete Vertrauenserwartungen bilden. Auf Grund dieser Vertrauenserwartungen werden beiderseits die Vertrauenshandlungen erbracht, die wiederum die Vertrauenswürdigkeit verstärken und die Vertrauenserwartung rechtfertigen. Dieser Zusammenhang ist in Abbildung 4 schematisch dargestellt.

So entsteht eine Situation in der sich die Kooperateure einander gegenseitig ausliefern und so die Opportunismusneigung ihres Gegenübers reduzieren. Es wurde zusammen der Wert eines maximenbasierten Vertrauensverhältnisses geschaffen, den keiner der Beteiligten durch defektives Verhalten gefährden möchte. 
Abb. 4: Entstehungsprozess eines maximenbasierten Vertrauensverhältnisses

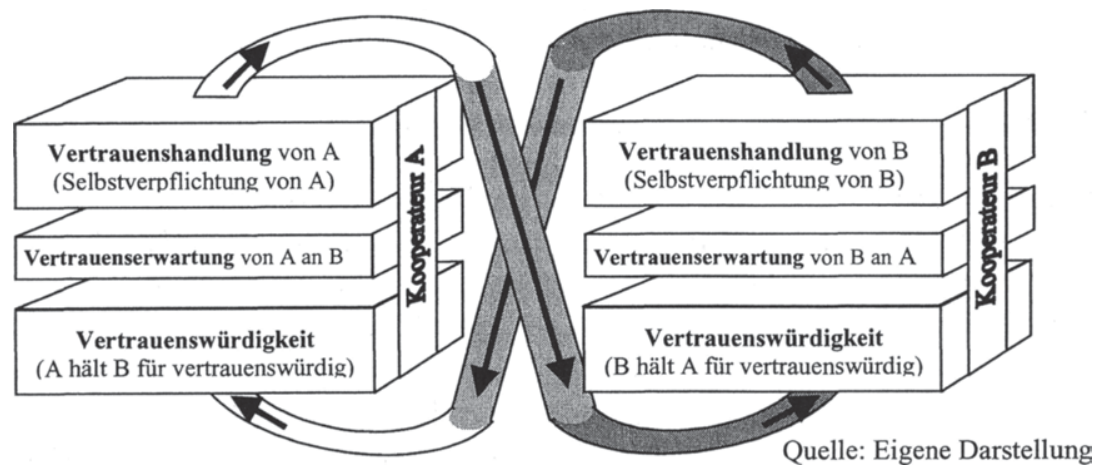

Obwohl der Opportunismusspielraum beim Aufbau eines maximenbasierten Vertrauensverhältnisses weiter in voller Größe bestehen bleibt, reduziert die Beschränkung der Opportunismusneigung der Beteiligten deren Verhaltensunsicherheit und absorbiert damit teilweise die Komplexität der Transaktionsbeziehung.

\subsection{Selbstverpflichtung als Möglichkeit zur Durchbrechung doppelter Kontingenz}

Besteht unter Kooperationspartnern ein maximenbasiertes Vertrauensverhältnis, so haben sich die Interakteure durch ihre Selbstverpflichtung auf die Maxime „Du sollst vertrauen" verpflichtet und sich damit für die Zukunft auf die Option „kooperatives Verhalten“ festgelegt. Diese Selbstverpflichtung des unvollständig informierten, begrenzt opportunistischen und begrenzt rationalen Akteurs bietet eine mögliche Lösung für soziale Dilemmata vom Typus des Gefangenendilemma an denen Komplexitätsreduktionsstrategien auf der Basis instrumentellen Vertrauens scheitern.

Das Gefangenendilemma ${ }^{186}$ beschreibt eine Situation in der zwei getrennt verwahrte Gefangene simultan zu einer Straftat verhört werden, zu der es keine Sachbeweise gibt. Dabei werden jedem von ihnen Vergünstigungen im Falle eines Geständnisses in Aussicht gestellt. Gesteht einer so wird er als Kronzeuge freigelassen und der andere wird zu einer langen Haftstrafe verurteilt. Gestehen beide, so wird beiden das Geständnis urteilsmildernd angerechnet. Kommt kein Geständnis zustande, würden beide Gefangenen wegen eines geringfügigen 
Delikts zu einer geringen Haftstrafe verurteilt. Keine der beiden weiß, wie sich der andere verhält. ${ }^{187}$ Sie befinden sich in einer Situation doppelter Kontingenz. ${ }^{188}$ Die Unsicherheit über das Verhalten des anderen führt dazu, dass die beiden Gefangenen versuchen, jeweils das für sie Beste aus der Situation zu machen. Das bedeutet, dass sie ihren persönlichen Schaden zu minimieren versuchen, unabhängig davon, wie sich ihr Interaktionspartner verhält. Sie verfolgen eine MaximinStrategie $^{189}$. In der Situation des Gefangenendilemmas bedeutet das, dass jeder der Gefangenen gesteht, um für sich im Falle eines Geständnisses des anderen zumindest eine Haftreduktion in Anspruch nehmen zu können. In der Spieltheorie kommt es somit, bei Unterstellung der üblichen Annahmen ${ }^{190}$, zu einem suboptimalen Ergebnis: Beide gestehen, beide werden verhaftet, beide werden aufgrund ihres Geständnisses zu einer Haftstrafe verurteilt. Beide wählen die nicht kooperative Strategie. ${ }^{191}$ Hätten beide die kooperative Strategie gewählt, hätte also keiner gestanden so wären beide nur zu einer geringfügigen Haftstrafe verurteilt worden und es wäre zu einer pareto-optimalen Lösung ${ }^{192}$ gekommen. Hätte ein Akteur kein Geständnis abgelegt, dann hätte er sich jedoch dem Risiko ausgesetzt, durch ein Geständnis seines Mithäftlings verurteilt zu werden und noch dazu keine Vergünstigungen zu bekommen. Das wäre für ihn die schlechteste aller Lösungen. Das Gefangenendilemma scheint unter den üblichen Annahmen der Spieltheorie nicht lösbar zu sein. ${ }^{193}$ Auch die Erweiterung des Spiels vom Typus des Gefangenendilemmas auf mehrere Durchgänge stellt auf Grund der Rückwärtsinduktion keine Lösung dar. ${ }^{194}$

187 Die Akteure verfügen über imperfekte Information. Sie sind nicht über die Strategiewahl des anderen informiert. Der Grund dafür liegt in der simultanen Strategiewahl.

188 Doppelte Kontingenz ist ,eine Situation, in der der andere immer anders handeln kann als ich erwarte, und [in der] er, gerade weil er weiß, was ich erwarte, anders handeln kann als ich erwarte.“ (Luhmann 1984, 179)

189 „Die Maximinstrategie eines jeden Spielers lässt sich ermitteln, wenn jeder Spieler Kenntnis über alle möglichen Strategiekombinationen sowie die resultierenden eigenen Auszahlungen hat. Die Maximinlösung beruht auf dem maximalen Ertrag, mit dem jeder Spieler im schlechtesten Fall rechnen kann. Jeder Spieler wählt diejenige Strategie, bei der er im schlechtesten Fall - d.h. bei der ungünstigsten Strategiekombination - die maximale Auszahlung erreicht (,Maximiere das Minimum"). Dies impliziert, dass Optimalitätsüberlegungen der Mitspieler nicht antizipiert werden und nicht in die eigene Strategiewahl einfließen." (Eberl 2002, 25)

1901. alle Teilnehmer sind vollkommen informiert, 2. die Akteure wählen ihre Strategie autonom und simultan, 3. alle Spieler verfügen über die selben Strategien, 4. keine dritte Partei kann die Vertragseinhaltung erzwingen.

191 vgl. Hardin 1971

192 Ein Zustand ist pareto-optimal (pareto-effizient) genau dann, wenn man ihn nicht so abändern kann, dass mindestens ein Akteur besser dasteht, ohne dass irgend ein anderer Akteur schlechter dasteht.

193 vgl. Wurche 1994

194 vgl. Engel 1999, 51 
Der Begriff Rückwärtsinduktion bezeichnet einen Mechanismus der dazu führt, dass die Akteure in einem Spiel mit mehreren Durchgängen, das durch doppelte Kontingenz gekennzeichnet ist, ab der ersten Runde die defektive Strategie wählen. Der einzige Grund für ein kooperatives Verhalten in einer solchen Situation besteht darin, dass man in der nächsten Runde auch ein kooperatives Verhalten des Interaktionspartners erwarten kann. In der letzten Runde besteht daher kein Grund mehr sich kooperativ zu verhalten. Erwarten die Akteure in der letzten Runde ein defektives Verhalten ihres Interaktionspartners, so besteht für sie auch in der vorletzten Runde kein Grund für kooperatives Verhalten mehr und sie wählen die defektive Strategie. Dieser Mechanismus setzt sich bis in die erste Runde fort, und führt dazu, dass sich die Akteure auch in Spielen mit mehreren Durchgängen von der ersten Runde an defektiv verhalten. ${ }^{195}$ Dieses Ergebnis gilt auch für den Fall, in dem das Ende des Spieles nicht bekannt ist, denn es besteht für den Akteur immer die Gefahr, dass sein Interaktionspartner einen möglichen Informationsvorsprung geltend machen kann. Er nimmt also ein fiktives Ende des Spieles an, um sein Risiko zu begrenzen. Damit wird jedes Spiel über mehrere Runden zu einem endlichen Spiel bei dem die Rückwärtsinduktion ein kooperatives Verhalten der Akteure verhindert.

Um eine Lösung des Gefangenendilemmas zu ermöglichen, müssen die Annahmen der Spieltheorie über die Akteure gelockert werden. ${ }^{196}$ Wir kehren wieder zum unvollständig informiert, begrenzt rational und begrenzt opportunistischen Akteur zurück. Nun tut sich eine Möglichkeit auf, soziale Dilemmata vom Typ des Gefangenendilemmas zu lösen. Den Schlüssel dazu stellt der Aufbau eines maximenbasierten Vertrauensverhältnisses dar.

In einer Situation, in der zwischen den beiden Häftlingen ein maximenbasiertes Vertrauensverhältnis besteht, erwarten beide Akteure ein loyales Verhalten ihres Mithäftlings, gestehen nicht und können so das pareto-optimale Ergebnis herbei-

195 Auch bei wiederholten Spielen defektieren die Akteure auf Grund der Rückwärtsinduktion bereits ab der ersten Runde. (vgl. Pettit/Sugden 1989 sowie Selten 1978)

196 „Eine Eigenart des Gefangenendilemmas besteht darin, dass die dominante egoistische Strategie beider Gefangenen zu einem individuellen Wohlfahrtsniveau für jeden der beiden Gefangenen führt, das geringer ist als jenes, welches die Gefangenen erreichen könnten, wenn sie ihren Entscheidungen eine nicht-egoistische Präferenzenordnung unterlegen. Das Argument hat eine eigentümliche Struktur: Es zeigt, dass der Altruist, der sich hier dadurch auszeichnet, dass er nicht ausschließlich das Ziel der Wohlfahrtsmaximierung verfolgt, dennoch im Resultat, dem faktisch erreichten Wohlfahrtsniveau, besser gestellt ist als der Egoist. Man kann also immer noch behaupten, dass sich auf die Dauer nur solches Verhalten durchsetzt, das auf welchem Wege auch immer zu einer Maximierung der Wohlfahrt führt; dies impliziert aber nicht, dass diesem Verhalten zwangsläufig egoistische Motive zugrundeliegen müssen.“(vgl. Zundel 1990, 37) 
führen. Durch die gegenseitige Vertrauenserwartung wird die Verhaltensunsicherheit absorbiert und die doppelte Kontingenz durchbrochen. Die Wahl der kooperativen Strategie (nicht gestehen) stellt eine risikoreiche Vorleistung des Akteurs dar, der sich dadurch auf der Basis seiner Vertrauenserwartung, seinem Interaktionspartner ausliefert. Diese Vertrauenshandlung (Selbstverpflichtung auf die Maxime „Du sollst vertrauen") beider Akteure verhindert das Entstehen der Dilemmasituation. Beide Akteure bilden aufgrund der Vertrauenswürdigkeit, die sie ihren Interaktionspartnern unterstellen, eine Vertrauenserwartung (der andere wird sich kooperativ verhalten), die die Basis für ihre Vertrauenshandlung (nicht gestehen) darstellt. Keiner der beiden Gefangenen gesteht. Beide werden nur zu einer geringfügigen Haftstrafe verurteilt. Das pareto-optimale Ergebnis ist erzielt.

Abb. 5: Maximenbasiertes Vertrauen ermöglicht Transaktionsbeziehungen

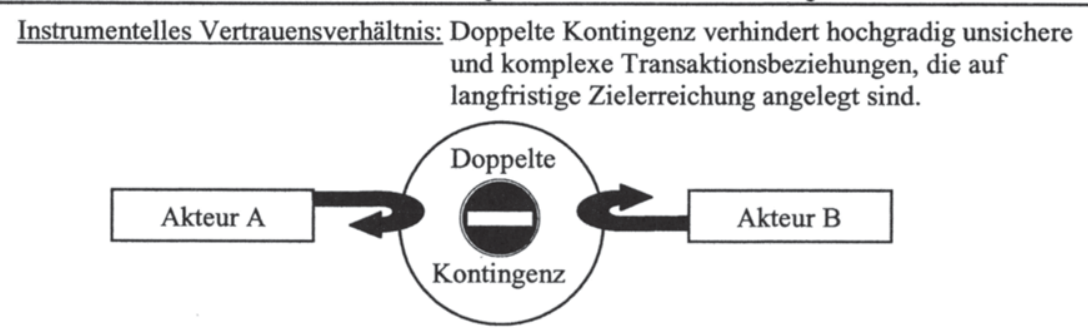

Maximenbasiertes Vertrauensverhältnis: Durchbrechung der Doppelten Kontingenz durch Selbstverpflichtung

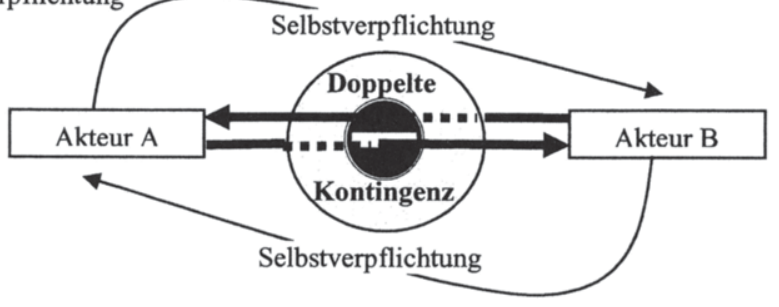

Quelle: Eigene Darstellung

Analog dazu ermöglicht der Aufbau eines maximenbasierten Vertrauensverhältnisses durch seine komplexitätsabsorbierende Wirkung den Aufbau und Bestand langfristiger komplexer Transaktionsbeziehungen, die auf der Basis instrumentellen Vertrauens nicht statt fänden. Denn 
„weder der Markt noch die hierarchische Organisation [sind] geeignete Koordinationsmechanismen, wenn eine auf langfristige Vorteile abzielende Transaktion hochgradig unsicher und komplex ist, sodass ein hierarchisches Festlegen des Verhaltens der Unsicherheit nicht angepasst wäre. D.h., einerseits steht die Langfristigkeit einer verlässlichen Koordination durch den Marktmechanismus entgegen, umgekehrt erlaubt die mangelnde Programmierbarkeit der Beziehung keine hierarchische Koordination." 197

Mit den Mitteln der graphischen Darstellung lässt sich der betroffene Teil der Transaktionen isolieren und die Situation in übersichtlicher Weise darstellen:

Abb. 6: Transaktionsbeziehungen und ihre Basen

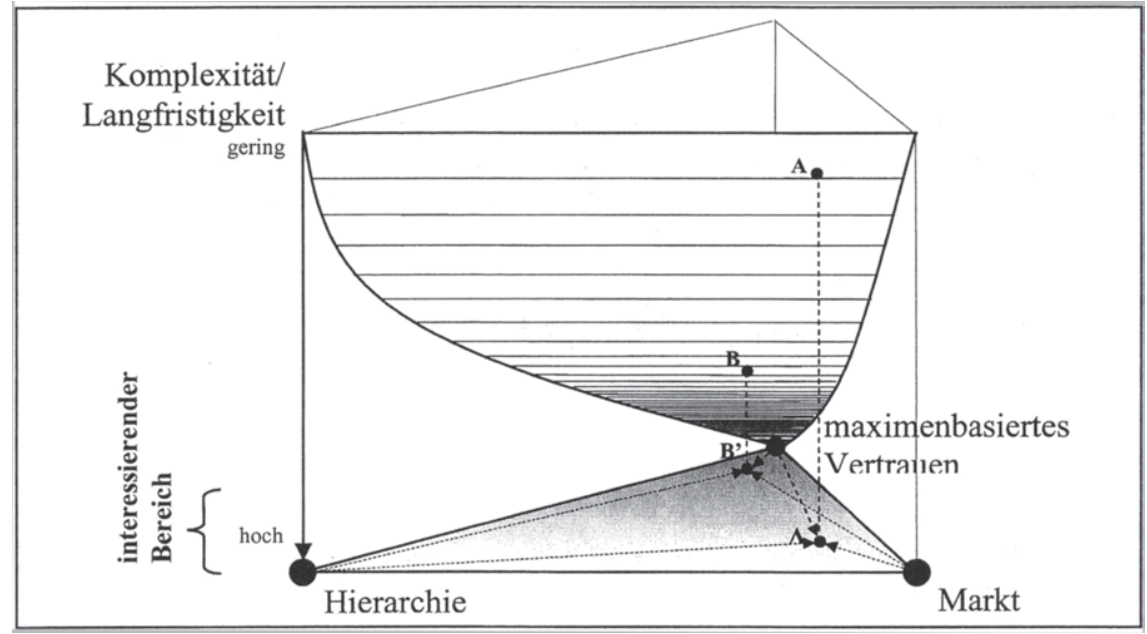

Quelle: Eigene Darstellung

Über das interne Verhältnis der drei Koordinationsformen notiert Rößl:

„Ebenso wie ,Markt' und ,Hierarchie' als idealtypische Endpunkte eines Kontinuums angesehen werden müssen, stellt auch ,Selbstverpflichtung' eine idealtypische Koordinationsform dar. Jede Transaktion ist somit eine Mischung aus ,Markt', ,Hierarchie' und ,Selbstverpflichtung'. “'198

Ein solches Mischverhältnis lässt sich durch eine Möglichkeitsfläche darstellen, die sich zwischen den Eckpunkten „Markt“, „Hierarchie“ und „maximenbasiertes Vertrauen“ aufspannt (Abbildung 6). Diese Fläche (Möglichkeitsfläche) ist dann die Summe aller Punkte von denen jeder jeweils ein mögliches Mischverhältnis der drei Koordinationsmechanismen bei einer konkreten Transaktion repräsentiert. 
Ergänzt man die Darstellung um eine dritte Dimension, so kann man auf dieser Achse die Komplexität und Langfristigkeit der Transaktionsbeziehung abbilden. Aus der Möglichkeitsfläche wird dann eine konkav gewölbte Fläche, die alle möglichen Kombinationen zwischen den Mischverhältnissen der Koordinationsmechanismen und der Komplexität bzw. Langfristigkeit der konkreten Transaktionsbeziehung abbildet. Die Wölbung der Fläche ergibt sich durch den überproportionalen Anstieg des Bedarfes an Koordinationsleistung auf der Basis maximenbasierten Vertrauens bei steigender Komplexität. Dieser wiederum ist durch den Transaktionskostenverlauf bei steigender Komplexität der Transaktionsbeziehungen begründet: Steigende Komplexität der Transaktionen führt zu einem überproportionalen Anstieg der Transaktionskosten sowohl bei Koordination durch den Marktmechanismus (bis hin zu Marktversagen) als auch bei Koordination durch hierarchische Steuerung (bis hin zu Organisationsversagen). ${ }^{199}$

So repräsentiert beispielsweise Punkt $\mathrm{A}$ in Abbildung 6 eine Transaktion, die durch eine geringe Komplexität und eine nicht langfristige Perspektive charakterisiert ist und daher $\mathrm{zu}$ ihrer Koordination weniger auf die Koordinationsleistung des maximenbasierten Vertrauens angewiesen ist. In der Abbildung dies wird durch den großen Abstand des Punktes A' (Projektion von A auf die Basisfläche) zum Eckpunkt „maximenbasiertes Vertrauen“ dargestellt. Punkt B stellt hingegen eine hochkomplexe und auf Langfristigkeit angelegte Transaktion dar. Eine solche Transaktionsbeziehung benötigt zu ihrer Koordination in weit höherem Ausmaß maximenbasiertes Vertrauen. ${ }^{200}$ Der Punkt B' ist daher (Projektion von B auf die Basisebene) nahe dem Eckpunkt „maximenbasiertes Vertrauen“ eingezeichnet.

Die vorliegende Arbeit konzentriert sich auf Transaktionsbeziehungen, die durch hohe Komplexität und eine langfristige Perspektive gekennzeichnet sind. Bezugnehmend auf Abbildung 6 geht es demnach um den unteren Bereich. Solche Transaktionsbeziehungen sind nämlich durch doppelte Kontingenz charakterisiert, deren Durchbrechung nur mittels maximenbasiertem Vertrauen nicht aber durch instrumentelles Vertrauen möglich ist. ${ }^{201}$

199 vgl. Röß1 1996, 315

200 vgl. Fink/Röß1 2004, 41

201 ,Grundsätzlich wird der Einsatz von Vertrauen insbesondere in Transaktionen von hoher Komplexität notwendig sein, bei denen die Erwartungen der Vertragsparteien nicht bzw. nur zu prohibitiv hohen Transaktionskosten explizit kontrahiert werden können. Dies trifft vor allem auf langfristige, sich über einen größeren Zeithorizont erstreckende Leistungsbeziehungen zu [...].“ (vgl. Ripperger 1998, 49) „Dort, wo explizite vertragliche Mechanismen nur zu prohibitiv hohen Transaktionskosten implementiert werden können, verbleibt als Organisationsprinzip nur Vertrauen zwischen den Transaktionspartnern." (vgl. Ripperger 1998, 50) 

4. Formulierung der Forschungsfrage und Arbeitshypothese

\subsection{Forschungsfrage}

\subsubsection{ZIEL DER ARBEIT}

Diese Arbeit setzt sich zum Ziel die Gültigkeit der in Kapitel 3 formulierten Argumentation empirisch zu untersuchen. Es soll erhoben werden, ob sich ein empirischer Nachweis erbringen lässt, für den Zusammenhang zwischen der Selbstverpflichtung der beteiligten Interaktionspartner und dem Erfolg, der an Synergiesystemen beteiligten Unternehmen. Es interessiert hier nicht der Erfolg auf Ebene des gesamten Synergiesystems sondern der Erfolg auf Unternehmensebene.

\subsubsection{WARUM DIE EXISTENZ VON ERFOLGSFAKTOREN VERMUTET WIRD}

Gäbe es bei Kooperationen zwischen zwei Unternehmen weder Faktoren, deren Beachtung die Erfolgswahrscheinlichkeit der Unternehmung erhöhen, noch solche Faktoren, die den Erfolg tendenziell vermindern, so müsste jede Kooperation gleich erfolgsträchtig sein. Da Kooperationen in der Realität aber von höchst unterschiedlichem Erfolg gekennzeichnet sind, ist davon auszugehen, dass eben diese gesuchten erfolgskritischen Faktoren bei Kooperationen existieren.

\subsubsection{SELBSTVERPFLICHTUNG ALS ERFOLGSFAKTOR IN SYNERGIESYSTEMEN}

- Ist nun die Selbstverpflichtung eine notwendige Voraussetzung für die Etablierung eines maximenbasierten Vertrauensverhältnisses und

- ist der Bestand eines maximenbasierten Vertrauensverhältnisses eine notwendige Voraussetzung für die Durchbrechung der doppelten Kontingenz und

- ist das wiederum eine notwendige Voraussetzung für die Etablierung und den Bestand von Transaktionsbeziehungen, die auf langfristige Vorteile abzielen und hochgradig unsicher und komplex sind,

- dann muss die Selbstverpflichtung der Interakteure ein erfolgskritischer Faktor einer solchen Transaktionsbeziehung sein und sich somit auf den Erfolg der beteiligten Unternehmen auswirken.

Die vorliegende Arbeit konzentriert sich bei der empirischen Untersuchung auf Synergiesysteme als Koordinationsform hochgradig komplexer und unsicherer Transaktionsbeziehungen, die auf langfristige Zielerreichung angelegt sind. 
Abb. 7: Argumentationsweg und Forschungsfrage

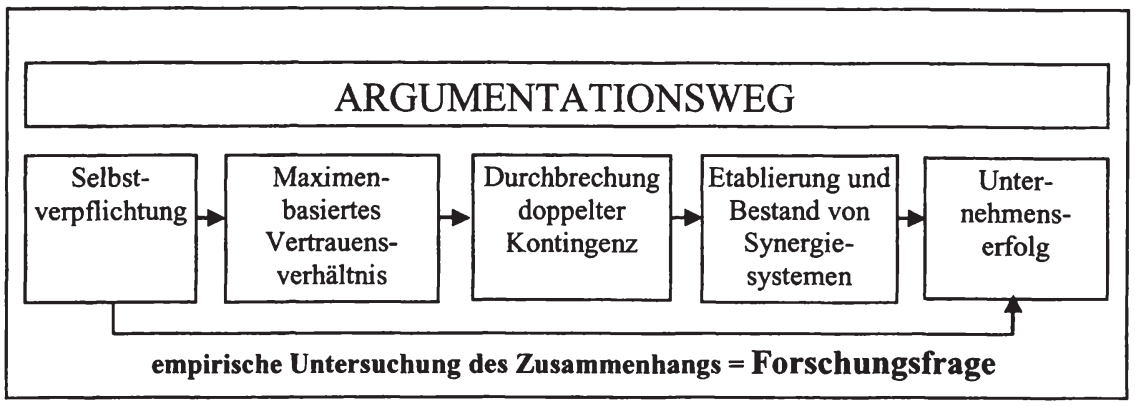

Quelle: Eigene Darstellung

Rößl sieht ebenfalls in der Selbstverpflichtung der Kooperationspartner einen erfolgskritischen Faktor bei Kooperationen im Sinne von Synergiesystemen. Er argumentiert, dass bei komplexen Transaktionen mit langfristiger Orientierung und unter großer Unsicherheit (z.B. Synergiesysteme), sowohl Marktmechanismus $^{202}$ als auch hierarchische Programmierung ${ }^{203}$ als Koordinationsmechanismen versagen. Solche Transaktionen finden dennoch statt und führen potentiell zum Erfolg, wenn die Kooperationspartner durch Selbstverpflichtung die doppelte Kontingenz durchbrechen. ${ }^{204}$ Somit ist die Selbstverpflichtung der an einer solchen Transaktion Beteiligten eine notwendige Voraussetzung für den Erfolg des Synergiesystems und der beteiligten Unternehmen.

\subsubsection{SELBSTVERPFLICHTUNG ALS ERFOLGSFAKTOR IN SYNERGIESYSTEMEN UNTER UNTERNEHMEN}

Die Etablierung und der Bestand von Synergiesystemen ist unter der Beteiligung verschiedenster Akteure denkbar. So können hochgradig komplexe und unsichere Transaktionsbeziehungen zwischen zwei Mitarbeitern einer Unternehmung bestehen, die gemeinsam ein langfristiges Ziel verfolgen. Ebenso kann die Ehe als Synergiesystem analysiert werden. Die vorliegende Arbeit beschränkt sich jedoch auf die Untersuchung von Synergiesystemen zwischen Unternehmen. Der Grund dafür liegt nicht so sehr darin, dass es sich beim vorliegenden Text um eine wirtschaftswissenschaftliche Arbeit handelt. Das ist eher die Folge, nicht aber die Ursache. Der Grund für die Beschränkung der vorliegenden Arbeit auf Unternehmen

202 Der Marktmechanismus mit seiner kurzfristigen Perspektive scheitert an der langfristigen Orientierung von Synergiesystemen.

203 Die Interdependenzen in einem Synergiesystem sind zu komplex, um von einer Programmierung erfasst zu werden.

204 vgl. Rößl 1994b, $19 f$. 
liegt vielmehr darin, dass das Wissen über den erfolgskritischen Charakter von Selbstverpflichtung gerade bei Transaktionsbeziehungen zwischen Unternehmen noch kaum Eingang in die Gedankenwelt der Wirtschafts- und Sozialwissenschaften gefunden hat. Niemand würde ernsthaft bezweifeln, dass die Selbstverpflichtung der Ehepartner ein wichtiger Faktor für das Gelingen einer modernen ${ }^{205}$ Ehe und somit einen Erfolgsfaktor für eine gelungene Lebensführung der Ehepartner ist. Die Frage nach dem Zusammenhang zwischen der Selbstverpflichtung der an einem Synergiesystem beteiligten Unternehmen und dem Erfolg dieser Unternehmen ist hingegen bisher weit weniger deutlich herausgearbeitet worden.

\subsubsection{SELBSTVERPFLICHTUNG ALS ERFOLGSFAKTOR BEI SYNERGIESYSTEMEN UNTER KMU}

Die vorliegende Arbeit wählt aus der Menge der Unternehmen die Gruppe der $\mathrm{KMU}^{206}$ für die empirische Untersuchung aus, denn der Koordination durch Selbstverpflichtung scheint gerade bei Transaktionsbeziehungen unter KMU besondere Bedeutung zuzukommen. Das liegt daran, dass in den KMU die Evolutionsbedingungen für Synergiesysteme tendenziell eher erfüllt sind als in Unternehmen anderer Größe. ${ }^{207}$

So hat das Management in KMU tendenziell einen langfristigen Zeitbezug und eine langfristige Nutzenorientierung. Zum einen erzwingt ein, für KMU typischer, begrenzter Absatzmarkt langfristige Kundenbeziehungen, was dazu führt, dass nach Auftragserteilung nicht nur der Prinzipal vom Leistungswillen des Agenten, sondern auch der Agent von der guten Nachrede des Prinzipals (Reputation) abhängt. KMU sind in ein regionales soziales Umfeld eingebettet. Die räumliche und physische Nähe zum Transaktionspartner bedingt vergleichsweise geringe Kommunikationsbarrieren und erleichtert so die Konsensbildung und die „Kommunikation einer konsistenten vertrauensermöglichenden Selbstdarstellung “208.

205 Die klassische Ehe wo der Vater seine Tochter in die Obhut des Ehemannes übergibt, stellt eher ein Hierarchiesystem als ein Synergiesystem dar.

$206 \mathrm{Im}$ vorliegenden Projekt erfolgt die Definition des Begriffs „Klein- und Mittelbetriebe (KMU)“, laut Empfehlung der Europäischen Kommission vom 3. April 1996 an die Mitgliedstaaten, die Europäische Investitionsbank (EIB) und an den Europäischen Investitionsfonds (EIF): KMU umfasst Betriebe mit weniger als 250 Beschäftigten, deren Jahresumsatz unter 40 Mio. Euro und deren Bilanzsumme unter 27 Mio. Euro liegt. Außerdem dürfen nicht mehr als $25 \%$ des Kapitals des betroffenen Betriebes im Besitz von einem oder mehreren Unternehmen gemeinsam stehen, welche die Definition der KMU nicht erfüllen.

Zur Abgrenzung des Unternehmenstyps „Klein- und Mittelbetriebe“ siehe: Mugler 1999a, 18ff.

207 Rößl 1994b, 26

208 Rößl 1994b, 28 
Zum anderen zielt das Management von KMU eher nicht auf kurzfristige Maximierung des Aktienwertes durch wechselnde Vorstandsgenerationen ab, sondern ist um den Erhalt der langfristigen Einkommensquelle für Generationen innerhalb des angestammten Marktes bemüht. Das Managementdenken in KMU zeichnet sich daher durch eine geringere Abzinsung der Zukunft aus. ${ }^{209}$

Weitere Ursachen für die vergleichsweise gute Erfüllung der Evolutionsbedingungen für Synergiesysteme in KMU sind deren Struktur und die Komplexität ihrer Absatzgüter. Einerseits verhindert die Struktur der KMU eine tayloristische Zerlegung der Arbeit, andererseits steht die Komplexität der Absatzgüter der lückenlosen Programmierung ihrer Erstellung entgegen. Detaillierte Anweisungen werden dadurch unmöglich. Globalanweisungen wiederum schaffen für die Austauschpartner Freiräume. Lückenlose Kontrolle ist unmöglich. Das Erleben dieser Freiräume führt zu einem Wissen um die gegenseitige Abhängigkeit, was wiederum die Grundlage für ein Commitment für die Zielerreichung des Transaktionspartners darstellt. ${ }^{210}$ Außerdem zeigt sich, dass die hierarchische „Planung auf die Menschen im mittelständischen Unternehmen nicht passt und für die Verhältnisse in diesen Unternehmen nicht viel taugt “211. Auch in Großbetrieben haben die hierarchischen Strukturen nur eine unterstützende Funktion für die laufende Selbstorganisation. ${ }^{212}$ Es ist daher anzunehmen, dass die Evolutionsbedingungen für Synergiesysteme in KMU günstiger sind als in Unternehmen anderer Größenordnung, denn ,schließlich dürften Klein- und Mittelbetrieben im wesentlichen die Ressourcen fehlen, um eine Misstrauensorganisation aufzubauen und beherrschen zu können.“213

Die Operationalisierung der Forschungsfrage ergibt, um einige, oben besprochene, einschränkende Elemente ergänzt, die Arbeitshypothese, welche die Basis für die weitere Arbeit darstellt.

\subsection{Arbeitshypothese}

Selbstverpflichtung ist bei Unternehmenskooperationen im Sinne von Synergiesystemen zwischen zwei KMU ein Faktor, der in signifikantem Zusammenhang mit dem Erfolg der an der Kooperation beteiligten Unternehmen steht.

209 vlg. Rößl 1994b, 26

210 vlg. Rößl 1994b, 26

211 Kirsch 1983, 399f

212 vlg. Kieser 1994, 206; Sydow 1992, 247 sowie Kasper 1990, 379

213 Röß1 1994b, 27 


\subsection{Forschungsdesign und weitere Entwicklung des Textes}

Bisher wurde aus der Forschungsfrage (Abschnitt 4.1.) eine Arbeitshypothese (Abschnitt 4.2.) formuliert, die es nun im Weiteren $\mathrm{zu}$ operationalisieren und $\mathrm{zu}$ überprüfen gilt. Abbildung 8 stellt das hierfür entwickelte Forschungsdesign als Flow-Chart der Arbeitsschritte des Projekts bzw. der Kapitel im Text in übersichtlicher Weise dar. Darüber hinaus gibt Abbildung 8 Auskunft darüber, welche Passagen des Texts sich mit der Formulierung von Hypothesen bzw. Fragen beschäftigen und welche der Überprüfung dieser Hypothesen bzw. der Beantwortung der Fragen gewidmet sind.

Abb. 8: Entwicklung der Kapitel im Text und der Arbeitsschritte des Projekts

\section{Entwicklung Entwicklung \\ der Kapitel der Arbeitsschritte \\ im Text des Projekts}

\section{1.}

4.2 .

5.1.

5.2 .

5.3.

5.4 .

5.5.1.

5.5.2.

5.6.1.-5.6.8.

5.6.9.

5.7.1.

5.7.2.1.

5.7.2.2.

5.7.3.

6.
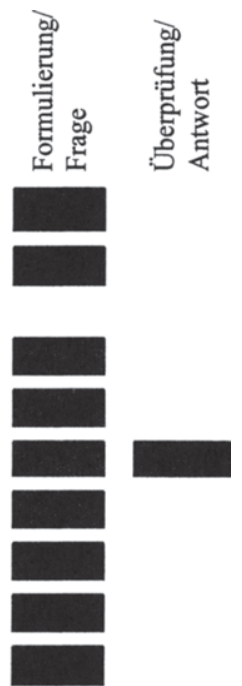

\begin{tabular}{|c|}
\hline Latente Variable \\
\hline Dimensionen der latenten Variablen (vorläufig) \\
\hline Qualitative Erhebung (Expertengespräche) \\
\hline
\end{tabular}

Dimensionen der latenten Variablen (endgültig)

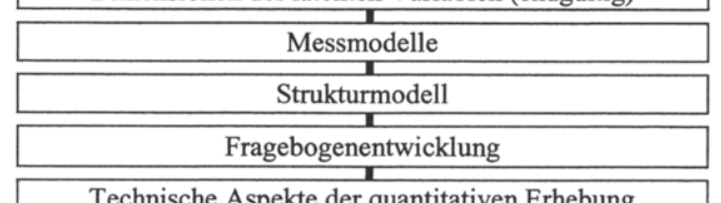

Technische Aspekte der quantitativen Erhebung

Durchführung der quantitativen Erhebung

\begin{tabular}{|c|}
\hline Überprüfung der Güte des Fragebogens \\
\hline Überprüfung der Gültigkeit der Messmodelle \\
\hline Überprüfung der Gültigkeit des Strukturmodells \\
\hline Überprüfung der Arbeitshypothese \\
\hline Beantwortung der Forschungsfrage \\
\hline
\end{tabular}

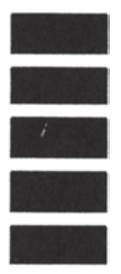

Quelle: Eigene Darstellung 



\section{Die empirische Erhebung}

\subsection{Variablen und ihre Dimensionen}

Da weder die Werte der Kategorisierungsvariablen (Synergiesystem), noch die der abhängigen Variablen (Unternehmenserfolg) und schon gar nicht die Werte der erklärenden Variablen (Selbstverpflichtung) in der empirischen Erhebung direkt erfragt werden können, ist es notwendig diese drei latenten Variablen in Dimensionen zu zerlegen, die erfragbar sind. So kann auf die Werte der latenten Variablen rückgeschlossen werden.

Im Folgenden werden in einem ersten Schritt aus jeder der drei Variablen die Dimensionen deduziert mittels derer, im Zuge der empirischen Erhebung, die Werte der Variablen erfasst werden sollen. In einem zweiten Schritt werden die vorläufigen Dimensionen im Zuge einer Expertenbefragung getestet und ergänzt. $\mathrm{Zu}$ den endgültigen Dimensionen werden dann Messvariablen definiert. Für jede Messvariable sind sodann Fragen zu formulieren, sodass mit der folgenden Fragebogenerhebung indirekt die Werte der latenten Variablen erhoben werden können. Abbildung 9 stellt die einzelnen Operationalisierungsschritte in systematischer Weise dar.

\subsection{Die vorläufigen Dimensionen}

\subsubsection{Kategorisierungsvariable „Synergiesystem“}

Um die Charakteristika jenes Kooperationstyps klar zu definieren, der in weiterer Folge mit dem Begriff „Synergiesystem“ bezeichnet wird, ist es sinnvoll, ihn im Möglichkeitsraum der unternehmerischen Transaktionsumwelt zu verorten. $\mathrm{Zu}$ diesem Zweck sind Dimensionen zu identifizieren, anhand welcher das Synergiesystem von anderen Formen der Organisation zwischenbetrieblicher Transaktionsbeziehungen unterschieden werden kann. Einerseits werden dazu Dimensionen herangezogen, die Kooperationen von Hierarchiebeziehungen, informellen Beziehungen und Konzentrationen abgrenzen. Andererseits werden diese um Dimensionen ergänzt, die Synergiesysteme von anderen Kooperationstypen kontrastieren. Mit Blick auf die nachgelagerte quantitative Erhebung, kann es nur das Ziel dieses Abschnittes sein, eine definitorische Isolierung des Begriffs „Synergiesystem“ im Möglichkeitsraum mittels möglichst weniger Dimensionen und Merkmalsausprägungen zu bewerkstelligen. 
Abb. 9: Operationalisierungsschema

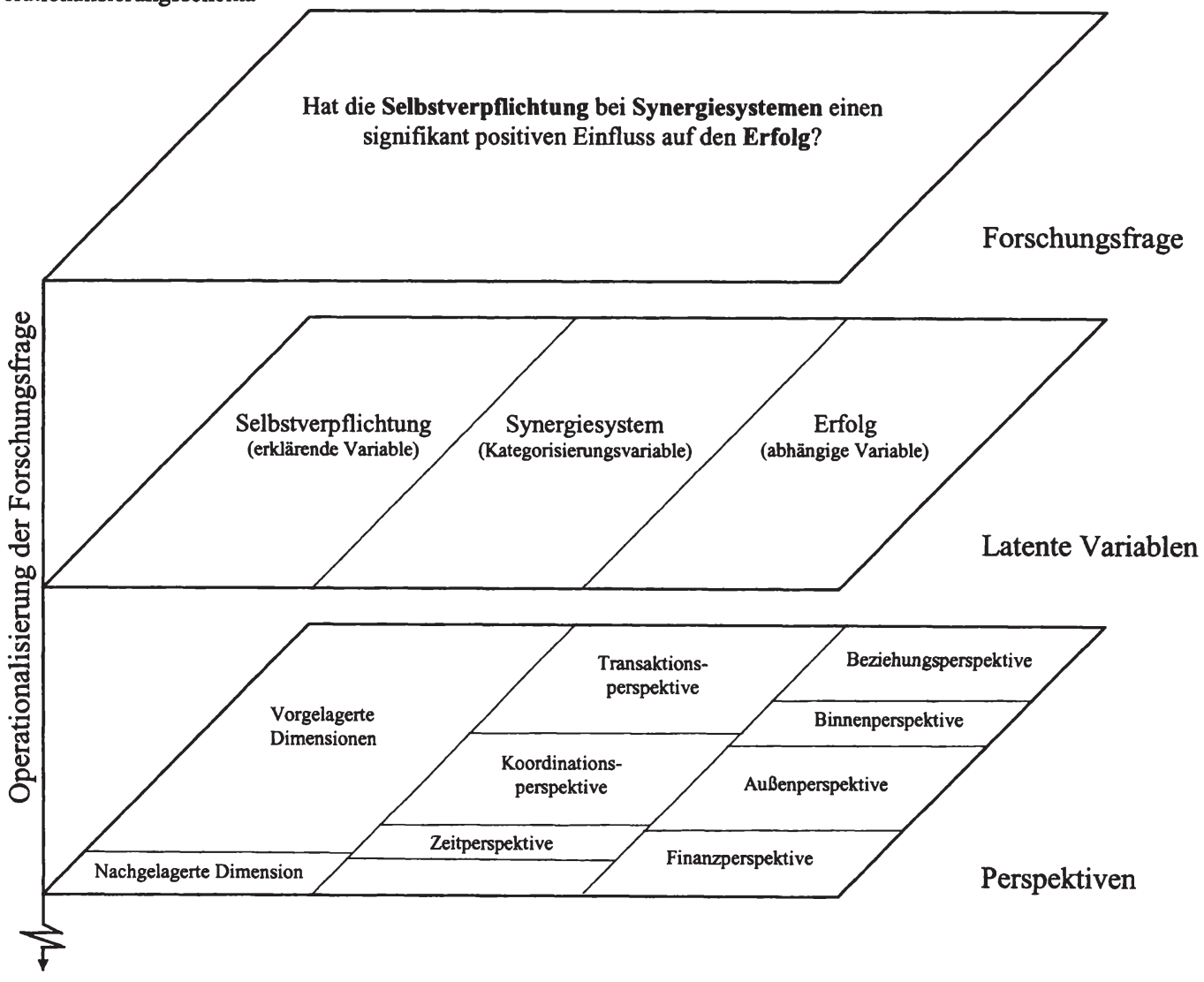




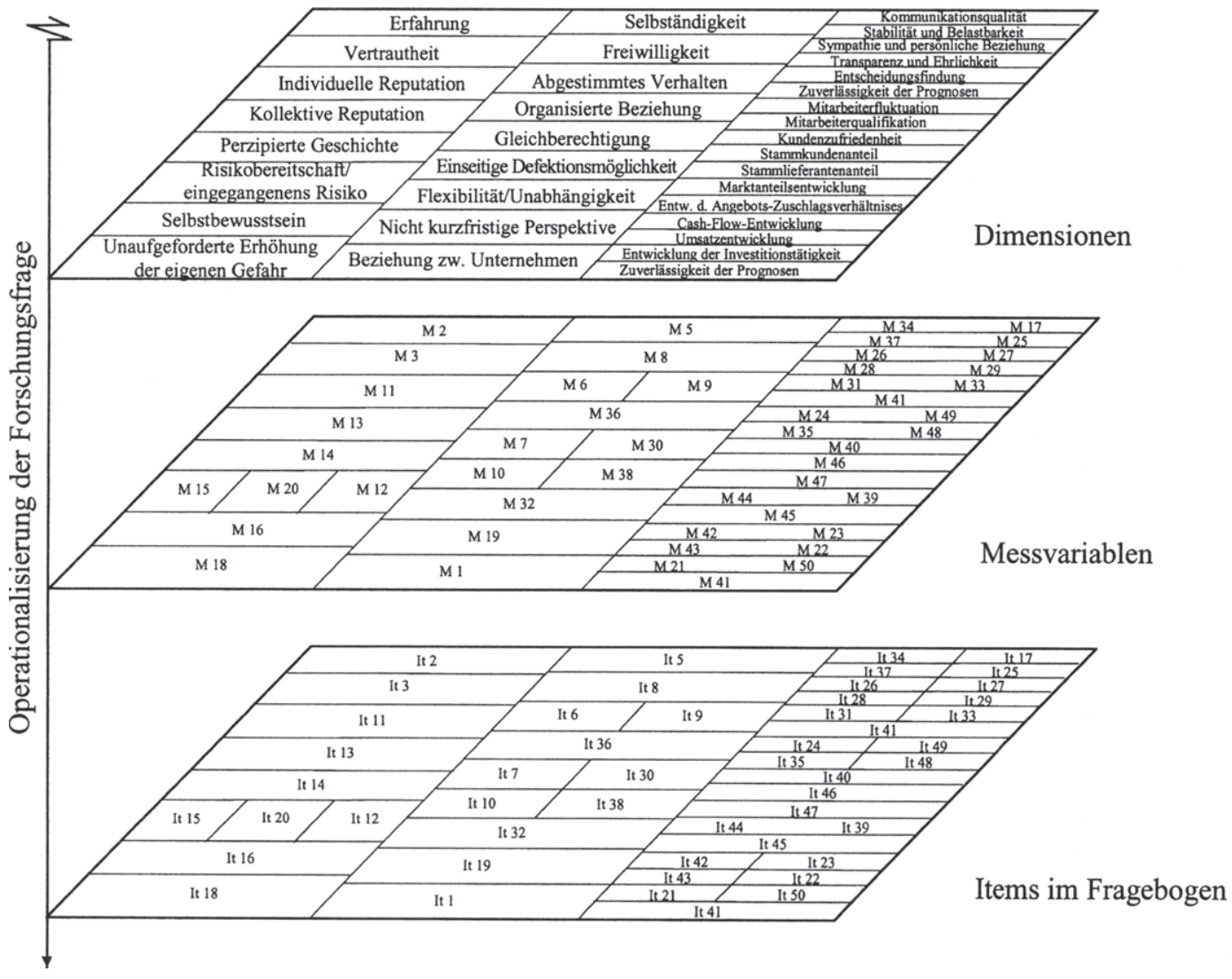




\subsubsection{Drei Perspektiven des Möglichkeitsraums}

Die Ausgestaltungsformen der Transaktionsbeziehungen werden an Hand von drei Perspektiven differenziert. Werden diese drei Perspektiven in einem Koordinatensystem einander zugeordnet, so spannen sie einen Raum auf, der alle möglichen Ausprägungskombinationen der, von den Perspektiven erfassten, Merkmale enthält. (siehe Abbildung 14)

Koordinationsperspektive - Diese Perspektive erfasst die Art der Koordination der an der Transaktionsbeziehung Beteiligten. Dabei stellen Koordination durch Selbstabstimmung bzw. Hierarchie die Extrema des Kontinuums dar, welches sich aus dieser Perspektive ergibt. In welcher Höhe die jeweilige Ausgestaltungsform der Transaktionsbeziehung eingetragen ist, gibt somit das für diese Form charakteristische Aus$\mathrm{ma} ß$ an hierarchischer Koordination bzw. Selbstabstimmung an.

Transaktionsperspektive - Aus dieser Perspektive wird das Ausmaß der Marktelemente bzw. Organisationselemente in einer Transaktion aufgetragen, das für die jeweilige Ausgestaltungsform der Transaktionsbeziehung typisch ist. Wandert man entlang der Transaktionsperspektive von links nach rechts, so nimmt das Ausmaß der Selbstorganisation in der typischen Transaktion in dem Maße ab, wie das Ausmaß der Organisationselemente zunimmt. ${ }^{214}$

Zeitperspektive - Die Zeitperspektive erfasst, wie stark sich die Beteiligten einer Transaktionsbeziehung an kurzfristigen Vorteilen orientieren. Je weiter man in die räumliche Tiefe des Möglichkeitsraums vordringt, umso mehr geben die Transaktionspartner ihre Orientierung an kurzfristigen Vorteilen zugunsten einer längerfristigen Sichtweise auf und verzichten auf kurzfristige Vorteile.

In Abbildung 10 werden nun die wichtigsten Ausgestaltungsformen von Transaktionsbeziehungen in den Möglichkeitsraum eingeordnet. Es zeigt sich, dass die drei eingenommenen Perspektive ausreichend sind, um die mit dem Begriff „Syn-

214 Die Vorstellung eines Kontinuums, entlang dessen jeder Punkt einem Mischverhältnis von Markt- und Organisationselementen in einer Transaktion entspricht und einem invers proportionalen Verhältnis von Markt- und Organisationselementen, ist Ausfluss des „Substitutionsgesetztes der Organisation" von Gutenberg. (vgl. Gutenberg 1958, S.49f.) 
ergiesystem" bezeichnete Ausgestaltungsform einer Transaktionsbeziehung (roter Würfel) von den anderen abzugrenzen.

- Abgrenzung des „Synergiesystems“ von der „Marktbeziehung“

Sowohl bei einer Marktbeziehung als auch in Synergiesystemen basiert die Koordination auf Selbstabstimmung. In Synergiesystemen ist jedoch das Ausmaß an Organisationselementen in einer typischen Transaktion weitaus größer als das bei Marktbeziehungen der Fall ist. Auch die zeitliche Orientierung der Handlungsträger unterscheidet die Marktbeziehung von einem Synergiesystem. Während Marktbeziehungen auf kurzfristige Vorteile abzielen, ist es ein wesentliches Merkmal eines Synergiesystems, dass die Transaktionspartner zugunsten einer längerfristigen Zielerreichung auf kurzfristige Vorteile verzichten.

- Abgrenzung des „Synergiesystems“ von der ,informellen Beziehung“

Bei der Abgrenzung informeller Beziehungen von Synergiesystemen ist die Zeitperspektive nur beschränkt hilfreich, da die Transaktionspartner informeller Beziehungen sowohl kurzfristige als auch längerfristige Ziele verfolgen können. Auch aus der Koordinationsperspektive kann keine Unterscheidung getroffen werden, denn bei beiden Ausgestaltungsformen basiert die Koordination der Transaktionsbeziehung auf Selbstabstimmung. Einzig die Transaktionsperspektive kann eine Unterscheidung leisten. Während informelle Beziehungen gerade dadurch gekennzeichnet sind, dass sie nur in sehr geringem Ausmaß Organisationselemente in einer typischen Transaktion enthalten, sind Synergiesysteme organisierte Beziehungen mit Systemeigenschaft.

- Abgrenzung des „Synergiesystems“ vom „Hierarchiesystem“

Beide, Hierarchiesystem und Synergiesystem, basieren auf einer langfristigen Orientierung der an der Transaktionsbeziehung beteiligten Handlungsträger. Auch das Verhältnis von Markt- und Organisationselementen in einer typischen Transaktion ist bei diesen beiden Ausgestaltungsformen einer Transaktionsbeziehung ähnlich. Der wesentliche Unterschied liegt in der Koordinationsform der Transaktionsbeziehung. Der Koordination durch Selbstabstimmung in einem Synergiesystem steht die hierarchische Koordination in einem Hierarchiesystem gegenüber. ${ }^{215}$ Daraus ergibt sich für das Hierarchiesystem eine starre Struktur an 
Kompetenzen während für das Synergiesystem gerade seine hohe Flexibilität charakteristisch ist.

- Abgrenzung des „Synergiesystems“ von der „Konzentration“

Mit der Konzentration als Ausgestaltungstyp von Transaktionsbeziehungen hat das Synergiesystem die langfristige Perspektive gemein. Der Anteil der Organisationselemente an einer typischen Transaktion ist jedoch bei der Konzentration wesentlich höher als bei Synergiesystemen. Außerdem unterscheiden sich diese beiden Arten der Gestaltung von Transaktionsbeziehungen durch ihre Koordinationsform. Konzentrationen bedingen eine hierarchische Koordination der Transaktionsbeziehungen während Synergiesysteme auf Selbstabstimmung der Kooperationspartner basieren. Der hohe Organisationsgrad und die hierarchische Koordination ermöglichen der Konzentration bei weitem nicht die Flexibilität die Synergiesysteme auszeichnet.

Abb. 10: Isolierung des Begriffs „Synergiesystem“ im Möglichkeitsraum

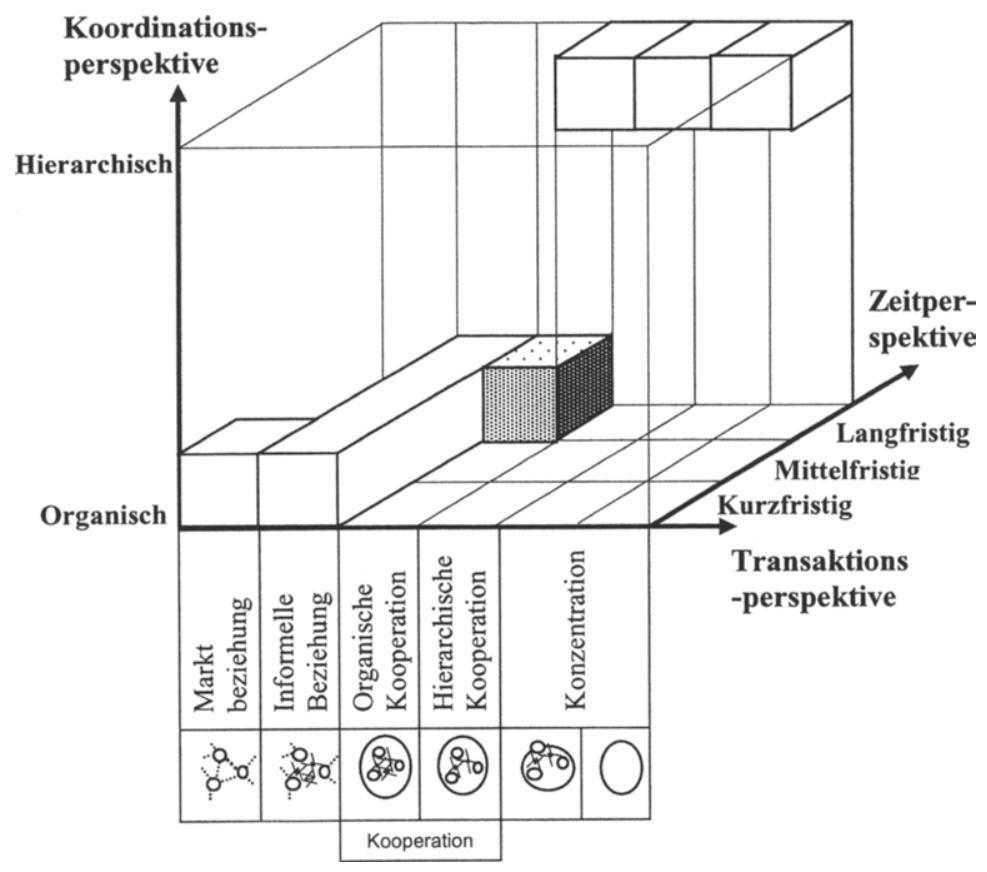

Quelle: Eigene Darstellung in Anlehnung an Kößl 1994a, 47 sowie Köbl 2000, 64. 


\subsubsection{Die Charakteristika des Synergiesystems}

Nachdem der Begriff Synergiesystem im Möglichkeitsraum verortet wurde, sind nun die Ausprägungen der aus den Perspektiven erfassten Merkmale herauszuarbeiten, die in ihrer Summe die Charakteristika eines Synergiesystems ausmachen. Da Synergiesysteme Kooperationen sind, werden zuerst die allgemeinen Charakteristika von Kooperationen erarbeitet, um dann das Synergiesystem anhand weiterer Unterscheidungsmerkmale unter den anderen Kooperationsformen identifizierbar zu machen.

\subsection{Charakteristika des Begriffs Kooperation}

Das erste Charakteristikum entspringt der Definition der Untersuchungseinheiten. Bei vorliegender Untersuchung sind die Untersuchungseinheiten Unternehmen. Daraus ergibt sich für die Arbeitsdefinition des Begriffs Kooperation folgende Einschränkung:

Beziehung zwischen Unternehmen - Damit sind vom Begriff Kooperation in vorliegender Arbeit nur jene Transaktionsbeziehungen erfasst bei denen die Beteiligten Unternehmen sind.

Aus der Transaktionsperspektive kann die Arbeitsdefinition des Begriffs Kooperation anhand folgender Charakteristika festgemacht werden:

Selbständigkeit - Alle Kooperationspartner haben formal-rechtliche Selbständigkeit, die dann erfüllt ist, wenn jedes an der Kooperation beteiligte Unternehmen jederzeit die Möglichkeit hat, auszuscheiden. ${ }^{216}$

Freiwilligkeit - Die Beteiligung an einer Kooperation ist für alle Kooperateure freiwillig und kann grundsätzlich im Rahmen einer vertraglichen Vereinbarung von den Vertragspartnern beendet werden. ${ }^{217}$

216 vgl. Tröndle 1987, 26

Wichtig dabei ist, dass es bei dem Kriterium Selbstständigkeit nicht um wirtschaftliche Selbständigkeit gehen kann, denn eine Kooperation deren Beendigung keine wirtschaftlichen Nachteile für die Kooperateure nach sich zieht, hat ihnen während ihres Bestandes keine wirtschaftlichen Vorteile gebracht. Eine Kooperation, die keine wirtschaftlichen Vorteile bringt, ist im Rahmen des wirtschaftlichen Denkmodells nicht sinnvoll.

217 vgl. Strohmayer 1996, 27. 
Während die Selbständigkeit der Transaktionspartner und die Freiwilligkeit ihrer Beteiligung an der Transaktionsbeziehung Kooperationen im Sinne der Arbeitsdefinition gegen stärker hierarchische Koordinationsformen zwischenbetrieblicher Transaktionsbeziehungen abgrenzt, können die Charakteristika „Abgestimmtes Verhalten“ und „Organisierte Beziehung“ zur Abgrenzung gegen stärker marktliche Transaktionsbeziehungen herangezogen werden.

Abgestimmtes Verhalten - Die Parteien einer Kooperation arbeiten aktiv zusammen, wobei unter aktiver Zusammenarbeit die Koordination von Funktionen und Aufgaben oder deren Übertragung auf gemeinsame Einrichtungen zu verstehen ist. ${ }^{218}$ Es geht dabei jedoch ausschließlich um aufeinander abgestimmtes Verhalten. Die Kooperateure müssen nicht das gleiche Ziel verfolgen. Die Mittel-Ziel-Hierarchien der einzelnen Unternehmen können voneinander abweichen. Wichtig ist, dass sie, welches Ziel sie auch immer verfolgen, zu Erreichung dieser Ziele die selben Mittel einsetzen. Es geht um die Parallelität bestimmter Aktivitäten, die mit einem Kooperationspartner zu koordinieren für die Kooperateure vorteilhaft ist.

Organisierte Beziehung - Die Austauschbeziehung zwischen den Kooperateuren besitzt Systemeigenschaft, dies setzt die Möglichkeit zur Selbstthematisierung voraus. ${ }^{219}$

Aus der Zeitperspektive lässt sich die Arbeitsdefinition des Begriffs Kooperation als Transaktionsbeziehung, die auf die Erreichung nicht kurzfristiger ${ }^{220}$ Ziele angelegt ist, gegenüber Transaktionsbeziehungen mit mittelfristiger oder kurzfristiger Perspektive abgrenzen.

Nicht kurzfristige Perspektive - Die Kooperationspartner verzichten zugunsten eines längerfristigen Vorteils auf kurzfristige Vorteile. ${ }^{221}$

218 vgl. Benisch 1973, 68

219 vgl. Plaßmann 1974, 23 und Rößl 1994a, 55

220 Die Formulierung „nicht kurzfristig“ soll auf den Umstand hinweisen, dass hier nicht notwendigerweise eine Frist von mehreren Jahren gemeint ist, sondern eine längere Frist als der Zeithorizont einer Markttransaktion darstellt.

221 vgl. Rößl 1994a, 54 


\subsection{Zusätzliche Charakteristika des Begriffs Synergiesystem:}

Nachdem es gelungen ist, die Arbeitsdefinition des Begriffs Kooperation von anderen Formen der Koordination von Transaktionsbeziehungen zu isolieren, hat innerhalb des hier verwendeten Kooperationsbegriffs noch eine Unterscheidung zwischen Synergiesystem und Hierarchiesystem stattzufinden. Zu diesem Zweck werden Charakteristika des Synergiesystems formuliert, die eine klare Unterscheidung aus der Koordinationsperspektive ermöglichen.

Gleichberechtigung/ohne Über- bzw. Unterordnung der beteiligten Subsysteme - Die Kooperationspartner sind gleichberechtigt und wechselseitig gleichgeordnet. Keiner der Partner übt eine hierarchische Steuerung aus. Kooperationen sind dadurch gekennzeichnet, „dass jeder Kooperationspartner Vorteile aus der eingegangenen Kooperation zieht.“222 Unter den Kooperationspartnern besteht annähernd Symmetrie bezüglich der Intensität der Kooperationsinteressen.

\section{Möglichkeit der einseitigen Defektion ${ }^{223}$ und Systemgefährdung ${ }^{224}$ - Jeder} Kooperationspartner in einem Synergiesystem kann durch sein Verhalten dem anderen Partnerunternehmen Schaden zufügen. Der Begriff Schaden beinhaltet im hier gebrauchten Sinn nicht nur materiellen sondern auch immateriellen Schaden. Somit kann jeder Kooperationspartner in einem Synergiesystem durch sein Verhalten das gemeinsame System gefährden.

Flexibilität ${ }^{225}$ - Die Handlungen der Kooperateure sind nicht von außen oder vom Kooperationspartner festgelegt. Jeder Kooperateur kann aus den ihm grundsätzlich offenstehenden Handlungsoptionen uneingeschränkt wählen.

Im Sinne der geforderten Übersichtlichkeit werden die identifizierten Charakteristika des Synergiesystems in Abbildung 11 nochmals zusammenfassend dargestellt.

222 Rühl 1980, 23

223 vgl. Plaßmann 1974, 23

224 vgl. Plaßmann 1974, 23

225 Die Flexibilität des Synergiesystems ist eine Folge des vergleichsweise niedrigen Organisationsgrades und der Koordination durch Selbstabstimmung und somit der Abwesenheit von hierarchischen Strukturen. 
Abb. 11: Zusammenfassende Darstellung der Dimensionen eines Synergiesystems
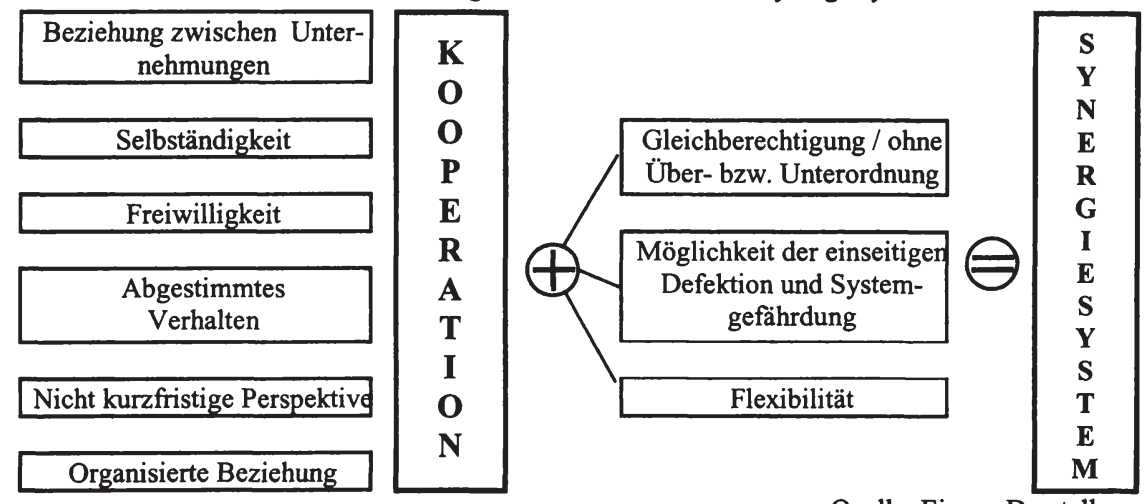

Quelle: Eigene Darstellung

\subsubsection{ERKLÄRENDE VARIABLE „SELBSTVERPFLICHTUNG“}

Bei der Erhebung der Selbstverpflichtung ist man mit den Unwegbarkeiten konfrontiert, die für alle Einstellungserhebungen typisch sind. Es erscheint daher als nicht sinnvoll, die Befragten direkt nach dem Grad ihrer Selbstverpflichtung zu fragen, da ein solches Vorgehen kaum zu brauchbaren Resultaten führte. Aus diesem Grund wird die Selbstverpflichtung im Zuge der quantitativen Erhebung im Rahmen dieses Projekts in indirekter Weise erhoben. Dazu werden Dimensionen formuliert, die der Selbstverpflichtung im Entstehungsprozess eines maximenbasierten Vertrauensverhältnisses vorgelagert bzw. nachgelagert sind und die daher in direktem Zusammenhang mit dem Zustandekommen bzw. den Resultaten der Selbstverpflichtung stehen. Sind einem die Werte dieser vor- bzw. nachgelagerten Dimensionen im Einstellungsspektrum eines Befragten bekannt, so ist der Rückschluss auf den Grad der Selbstverpflichtung dieses Befragten möglich. Die Rechtfertigung dieses Schlusses ist für jede Dimension einzeln zu erbringen. Sie findet daher direkt bei der jeweiligen Besprechung der jeweiligen Dimension statt.

\subsubsection{Vorgelagerte Dimensionen}

\subsection{Die Wirkung der Erfahrung auf das menschliche Verhalten}

Als theoretischen Hintergrund unserer Betrachtung der Wirkung der Erfahrung auf das Verhalten der Akteure im Allgemeinen und der Wirkung der Erfahrung auf die Selbstverpflichtung der Akteure im Speziellen, bietet uns Alfred Schütz's 
Konzeption über die Strukturen der Lebenswelt. ${ }^{226}$ Demnach werden die Erfahrungen mit denen der Akteur konfrontiert ist nach ihrer Relevanz gefiltert. Die thematische Relevanz bestimmt, ob eine Erfahrung in den thematischen Kern des Bewusstseins vordringt. Die Ausrichtung der thematischen Relevanz bestimmt die Motivationsrelevanz. Sie bestimmt, warum das Individuum seine Aufmerksamkeit in eine bestimmte Richtung lenkt. Die Motivationsrelevanz stellt so das Motiv für die Ausrichtung der Filterung nach der thematischen Relevanz bereit. Ist eine Erfahrung aus der Menge der möglichen Erfahrungen durch den Filter der thematischen Relevanz in den thematischen Kern des Bewusstseins eingedrungen, so wird es interpretiert, es wird nach der Interpretationsrelevanz auf Bestimmtheit und Vertrautheit geprüft und im Wissensvorrat abgelegt. Die Wissenselemente werden typisiert, das heißt, sie werden zu Ähnlichem zusammengefasst. Der so strukturierte Wissensvorrat bietet wiederum die Grundlage für die Filterung der Erfahrungen. Der individuelle Wissensvorrat wird vergesellschaftet und speist so den kollektiven Wissensvorrat. ${ }^{227}$

Es wurde nun skizziert, wie sich der Mensch durch Erfahrungen einen Wissensvorrat aneignet. In einem zweiten Schritt soll gezeigt werden, wie dieser Wissensvorrat das Verhalten der Individuen beeinflusst. Als Ausgangspunkt dazu dient uns die Feststellung, dass jede Handlung ihren Ursprung in einer Phantasie hat. Diese Phantasien konkretisieren sich durch die Konfrontation mit einem Ziel zu Entwürfen über mögliche Handlungen. Jeder, der so entstandenen Entwürfe wird nun auf seine Tauglichkeit zur Zielerreichung getestet. Dabei wird auf den Wissensvorrat zurückgegriffen. Es werden mit Hilfe der typisierten Erfahrungen aus dem Wissensvorrat Prognosen über die Erfolgswahrscheinlichkeiten der einzelnen Handlungsentwürfe aufgestellt. Aus der Menge der Handlungsentwürfe wird sodann der erfolgsträchtigste ausgewählt und realisiert. So wird Handeln zur Handlung. Dieser Vorgang wiederholt sich für jede Teilhandlung. Das heißt, das Individuum testet seine Handlung schon während des Handlungsverlaufes und nimmt bei Bedarf Änderungen vor. ${ }^{228}$

226 Schütz, A. Luckmann, T. (1979): Strukturen der Lebenswelt. Band 1, Frankfurt 227 Der kollektive wirkt auch auf den individuellen Wissensvorrat ein. Dies geschieht beispielsweise über gesellschaftliche Normen und Werte.

228 Schütz/Luckmann 1979, 16 f 
Abb. 12: Einfluss der Erfahrung auf das Verhalten

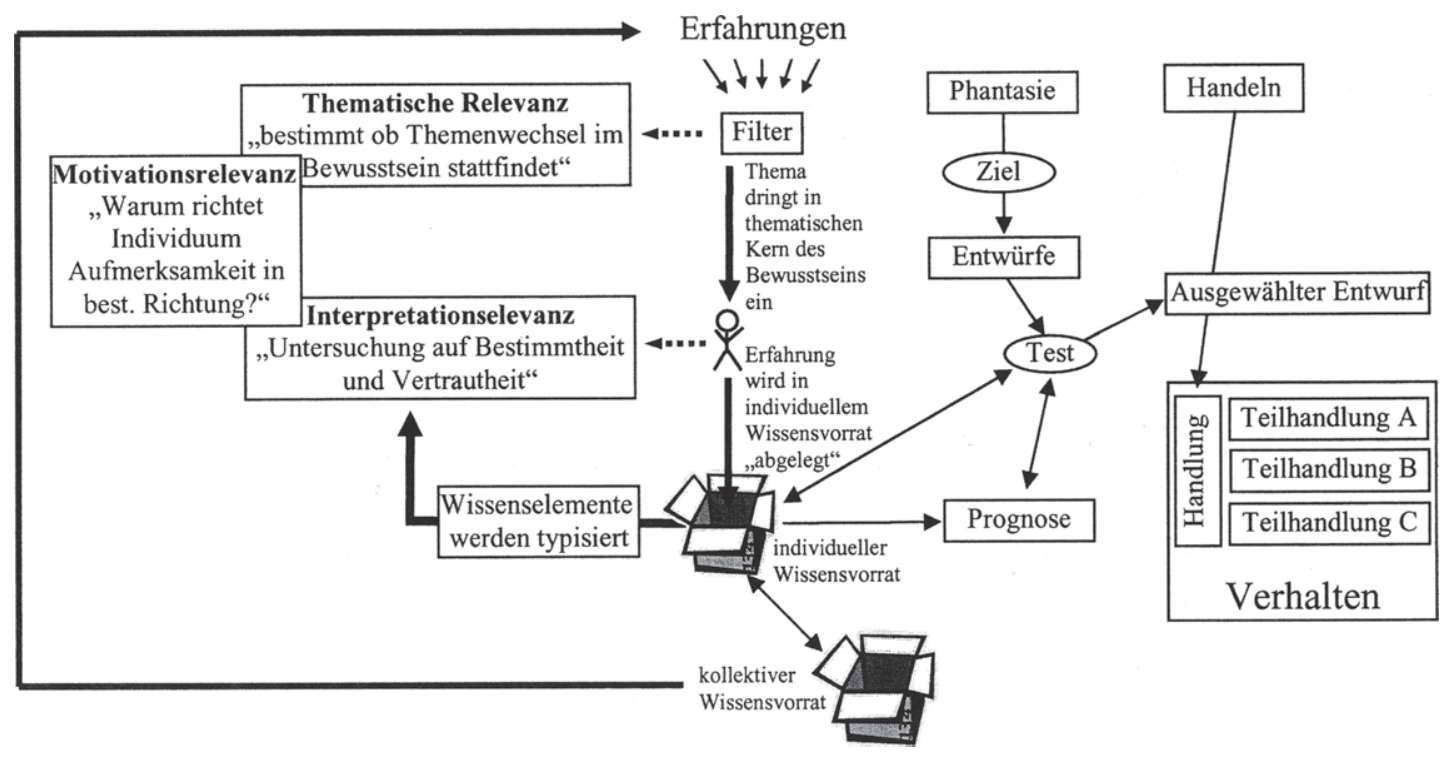


Zusammenfassend kann festgehalten werden:

„Der persönliche Wissensvorrat wird durch Wissenserwerb aufgebaut und besteht aus einzelnen Wissenselementen. Der Erwerb findet in Handlungen, sozialen Beziehungen, Erfahrungen statt und ist niemals abgeschlossen. Alles was vom Bewusstsein erfasst wird, verändert oder ergänzt den persönlichen Wissensvorrat. Auf der anderen Seite dient der Wissensvorrat zur Definition und Bewältigung von [zukünftigen] Situationen“.229

Auch der Einfluss der Erfahrung auf die Bereitschaft des Akteurs sich selbst auf die Maxime „Du sollst vertrauen“ festzulegen, erfolgt über den Wissensvorrat und seine entscheidende Rolle bei der Auswahl des zu realisierenden Handlungsentwurfes. Die Grundlage der Selbstverpflichtung ist die Einschätzung des Kooperationspartners als vertrauenswürdig. ${ }^{230}$ Die im Wissensvorrat strukturiert gesammelte Erfahrung bietet dem Akteur Informationen, an denen er sich bei der Einschätzung seines Interaktionspartners orientieren kann.

\subsection{Vertrautheit, Reputation und perzepierte Geschichte}

Neben der Erfahrung sind drei weitere - der Selbstverpflichtung vorgelagerte Dimensionen identifiziert worden: Vertrautheit, Reputation sowie perzepierte Geschichte. Es erscheint sinnvoll, ihren Einfluss auf die Selbstverpflichtung der Akteure parallel zu dem der Erfahrung zu besprechen, denn die Begriffe Erfahrung, Vertrautheit, Reputation und perzepierte Geschichte haben eine ähnliche Struktur (siehe Abbildung 13).

Sowohl Erfahrung, Reputation, Vertrautheit als auch perzepierte Geschichte stellen dem Akteur Informationen bereit, die seine Einstellungen gegenüber Kooperationspartnern beeinflussen. So wie bei der Erfahrung stellt auch bei der Vertrautheit das eigene Erleben die Quelle der Informationen dar. Im Unterschied $\mathrm{zu}$ der Erfahrung, bei der Informationen über Kooperationspartner aus der Vergangenheit typisiert werden und auf potentielle zukünftige Kooperationspartner übertragen werden, bietet die Vertrautheit Informationen über einen bestimmten Interaktionspartner in einer bereits bestehenden Transaktionsbeziehung. Das bedeutet, Vertrautheit bezeichnet Wissen über einen bestimmten Kooperationspartner, während Erfahrung Wissen über Kooperationspartner im Allgemeinen darstellt. 
Nicht nur die Struktur der Begriffe Erfahrung und Vertrautheit ist ähnlich, sondern auch die Wirkungsweise der Vertrautheit auf die Einschätzung der Vertrauenswürdigkeit eines Interaktionspartners ist analog zu der der Erfahrung. Rößl formuliert den Zusammenhang folgendermaßen: „A kann B vertrauen, weil A eine breite Informationsbasis [...] zur Stützung der These von der Vertrauenswürdigkeit von B hat. “'231 So nimmt die Vertrautheit Einfluss auf die Selbstverpflichtung des Akteurs.

Abb. 13: Vergleich und Abgrenzung der Begriffe Reputation, Vertrautheit und Erfahrung

\begin{tabular}{|c|c|c|c|c|c|}
\hline & \multicolumn{2}{|c|}{ Reputation } & \multirow[b]{2}{*}{ Vertrautheit } & \multirow[b]{2}{*}{$\begin{array}{l}\text { perzepierte } \\
\text { Geschichte }\end{array}$} & \multirow[b]{2}{*}{ Erfahrung } \\
\hline & kollektiv & individuell & & & \\
\hline $\begin{array}{c}\text { Informa- } \\
\text { tionsquelle }\end{array}$ & $\begin{array}{c}\text { sozialisiertes } \\
\text { fremdes } \\
\text { Erleben }\end{array}$ & $\begin{array}{c}\text { sozialisiertes } \\
\text { fremdes } \\
\text { Erleben }\end{array}$ & $\begin{array}{l}\text { eigenes } \\
\text { Erleben }\end{array}$ & $\begin{array}{c}\text { nicht sozial } \\
\text { isiertes fremdes } \\
\text { Erleben }\end{array}$ & $\begin{array}{l}\text { eigenes } \\
\text { Erleben }\end{array}$ \\
\hline $\begin{array}{c}\text { Information } \\
\text { über }\end{array}$ & $\begin{array}{c}\text { unbestimmten } \\
\text { Kooperations- } \\
\text { partner }\end{array}$ & $\begin{array}{c}\text { bestimmten } \\
\text { Kooperations- } \\
\text { partner }\end{array}$ & $\begin{array}{c}\text { bestimmten } \\
\text { Kooperations- } \\
\text { partner }\end{array}$ & $\begin{array}{c}\text { bestimmten } \\
\text { Kooperations- } \\
\text { partner }\end{array}$ & $\begin{array}{c}\text { unbestimmten } \\
\text { Kooperations- } \\
\text { partner }\end{array}$ \\
\hline
\end{tabular}

Quelle: Eigene Darstellung

Die perzepierte Geschichte stellt Informationen über einen bestimmten, am Markt tätigen Akteur dar. Diese Informationen sind nicht im Erleben von Individuen gespiegelt, insofern sind sie objektive Daten. Da die perzepierte Geschichte einen Informationstyp darstellt, der nicht sozialisiert ist und auch nicht durch eigenes Erleben erschlossen wird, muss der Akteur diese Information aktiv beschaffen.

Die Reputation wirkt auf ähnliche Weise wie die Erfahrung auf das Urteil des Akteurs über die Vertrauenswürdigkeit eines potentiellen Interaktionspartners. Es ist jedoch festzuhalten, dass die Reputation, anders als die Erfahrung, nicht auf das Selbstbewusstsein, sondern ausschließlich auf die Risikobereitschaft des Akteurs einwirkt. Man kann zwischen kollektiver Reputation und individueller Reputation unterscheiden. Kollektive Reputation stellt Informationen über eine Gruppe von Akteuren dar. Beispielsweise hat die Gruppe der Gebrauchtwagenhändler eine Reputation genauso wie auch die der Buchhalter. Individuelle Reputation ist gewissermaßen öffentliche Information über einen bestimmten im Markt tätigen Akteur. ${ }^{232}$ Die Quelle dieser Informationen ist sozialisiertes fremdes 
Erleben. Bei der Heranziehung der Reputation als Information über einen bestimmten potentiellen Interaktionspartner oder einen Interaktionspartner, der einer bestimmten Gruppe angehört, greift der Akteur auf den kollektiven Wissensvorrat zurück. Die Reputation transportiert sozialisierte fremde Erfahrungen und bietet so für den Akteur Orientierung bei der Einschätzung der Vertrauenswürdigkeit eines anderen Akteurs.

Zur Beurteilung der Vertauenswürdigkeit eines Unternehmens wird auch seine Verhaltensgeschichte herangezogen. Dabei kommt es wesentlich auf die Stabilität der dem Verhalten zugrunde gelegten Maximen an. Die perzipierte Geschichte hat wesentlichen Einfluss auf die Möglichkeit des Vertrauensaufbaus. ${ }^{233} \mathrm{Ob}$ der Akteur seinen Interaktionspartner als vertrauenswürdig einschätzt, bestimmt über seine Selbstverpflichtung.

\subsection{Risikobereitschaft und Selbstvertrauen}

Wer sich selbst der Maxime „Du sollst vertrauen“ verpflichtet, der legt sich für die Zukunft darauf fest, sich kooperativ zu verhalten. Diese Selbstverpflichtung basiert auf der Vertrauenserwartung, der Erwartung, dass der Interaktionspartner freiwillig auf opportunistisches Verhalten verzichtet und das in ihn gesetzte Vertrauen rechtfertigt. Dennoch besteht immer die grundsätzliche Möglichkeit, dass sich der Kooperationspartner defektiv verhält und die Vertrauenserwartung enttäuscht. Wer sich selbst zu kooperativem Verhalten verpflichtet, der geht das Risiko der Enttäuschung seiner Erwartung über das ebenfalls kooperative Verhalten seines Interaktionspartners ein. ${ }^{234}$ Ohne die Bereitschaft dieses Risiko einzugehen, kann Selbstverpflichtung nicht stattfinden.

Die Risikobereitschaft ist jedoch nicht voraussetzungslos. So setzt Risikobereitschaft das Selbstvertrauen voraus, dass der Akteur mit der Situation umgehen kann, die entsteht, wenn seine Vertrauenserwartung enttäuscht wird. ${ }^{235} \mathrm{Um}$ bereit zu sein, sich dem Risiko eines Vertrauensbruchs durch den Transaktionspartner auszusetzen, muss der Akteur auf seine eigene Stärke vertrauen. Er muss der Meinung sein, über genügend Ressourcen zu verfügen, um eine Enttäuschung seiner Erwartung über das kooperative Verhalten seines Interaktionspartners verkraften zu können. Somit setzt Selbstverpflichtung nicht nur Risikobereitschaft, sondern auch Selbstvertrauen voraus.

234 Siehe dazu Abschnitt 2.4. Das maximenbasierte Vertrauensverhältnis

235 vgl. Röß1 1994a, S.197 


\subsubsection{Nachgelagerte Dimension: Unaufgeforderte Erhöhung der eigenen Gefahr}

Während die vorgelagerten Dimensionen der Selbstverpflichtung auf die Entwicklung der Selbstverpflichtung einen entscheidenden Einfluss haben, stellt die nachgelagerte Dimension das erlebbare Resultat der erfolgten Selbstverpflichtung dar.

Für den Interaktionspartner wird die Selbstverpflichtung dadurch erlebbar, dass der Akteur seine Festlegung auf die Maxime „Du sollst vertrauen“ glaubhaft kommuniziert ${ }^{236}$. Er legt sich für die Zukunft auf die Option kooperatives Verhalten fest und eliminiert dadurch die Option defektives Verhalten aus seinem Verhaltensportfolio. Durch die Kommunikation seiner Selbstverpflichtung in einer Situation doppelter Kontingenz ermöglicht der Akteur die Entstehung eines maximenbasierten Vertrauensverhältnisses. Gleichzeitig stellt seine Selbstverpflichtung eine risikoreiche Vorleistung dar. Die Selbstverpflichtung des Akteurs schlägt sich somit in einer unaufgeforderten Erhöhung seiner eigenen Gefahr nieder. Der Gefahr nämlich, dass der Interaktionspartner die erbrachte, risikoreiche Vorleistung ausnützt und sich wider Erwarten defektiv verhält.

Abb. 14: Zusammenfassende Darstellung der Dimensionen der erklärenden Variablen „Selbstveroflichtung"

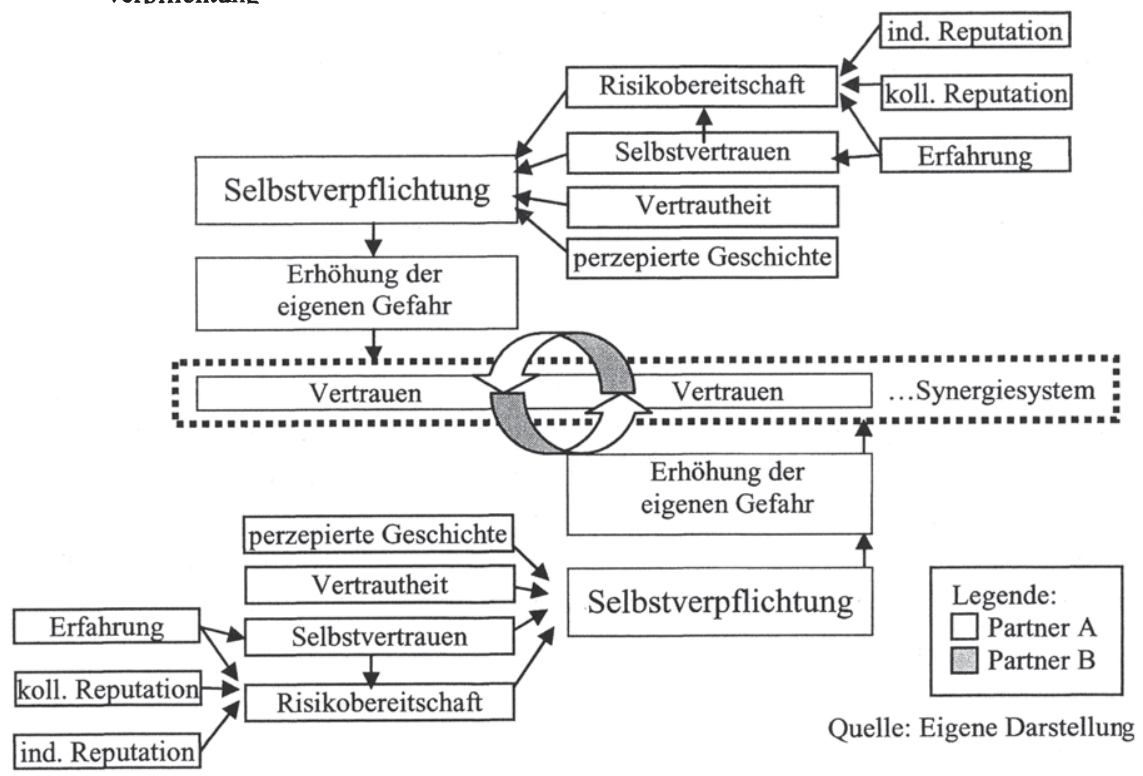

236 Das kann z.B. durch Handlungen geschehen. 
Um dem Anspruch auf Übersichtlichkeit gerecht zu werden, seien die Dimensionen der Variablen „Selbstverpflichtung“ in Abbildung 14 zusammenfassend dargestellt.

\subsubsection{ABHÄNGIGE VARIABLE „ERFOLG“}

Untersucht man Synergiesysteme zwischen Unternehmen, so kann man die Auswirkungen der Kooperationen auf den Erfolg auf zwei Ebenen betrachten. Die erste Möglichkeit besteht darin, den Erfolg auf der Ebene des Synergiesystems zu erheben. Das Ergebnis einer solchen Erhebung zeigt den Erfolg des Synergiesystems als Ganzes. Ist ein Synergiesystem erfolgreich, so wird das Auswirkungen auf die Performance der beteiligten Unternehmen haben. Damit ergibt sich eine zweite Möglichkeit den Erfolg eines Synergiesystems zu messen. Dazu wird der Erfolg der an einem Synergiesystem beteiligten Unternehmen herangezogen. Die Messung des Erfolgs findet auf der Ebene der Unternehmen statt, die an dem Synergiesystem beteiligt sind.

Die vorliegende Arbeit konzentriert sich auf die Erhebung des Erfolgs auf der Ebene des Unternehmens. Es soll dabei aber auf die Auswirkungen der Selbstverpflichtung auf den Erfolg der beteiligten Unternehmen aus mehreren Perspektiven eingegangen werden. Um diesem mehrdimensionalen Anspruch gerecht zu werden, wird für die Erhebung der Ausprägungen der abhängigen Variablen Erfolg auf die für vorliegende Zwecke adaptierte Struktur der Balanced Scorecard zurückgegriffen. The Balanced Scorecard

,is a set of measures that gives [...] a fast but comprehensive view of the business. [It] includes financial measures that tell the results of actions already taken. And it complements the financial measures with operational measures and the organisational measures on customer satisfaction, internal processes, and the organisation's innovation and improvement activities - operational measures that are the drivers of future financial performance.“ 237

Die hier verwendete Balanced Scorecard ist um die Innovationsperspektive gekürzt. Ergänzt wurde sie jedoch um die Beziehungsperspektive.

Es ergibt sich somit eine Balanced Scorecard, welche die Variable Erfolg aus vier Perspektiven beleuchtet und so eine umfassende Erhebung der Werte der Variablen Erfolg ermöglicht. ${ }^{238}$ Aus der Beziehungsperspektive wird die Qualität der Beziehung zwischen den an dem Synergiesystem beteiligten Unternehmen 
erhoben. Auf der Ebene der beteiligten Unternehmen wird der Erfolg aus drei Perspektiven betrachtet: Außenperspektive, Binnenperspektive und Finanzperspektive. Es ergibt sich für die Erhebung der abhängigen Variablen folgendes Bild:

Abb. 15: Struktur der Balanced Scorecard zur Erhebung der abhängigen Variablen Erfolg

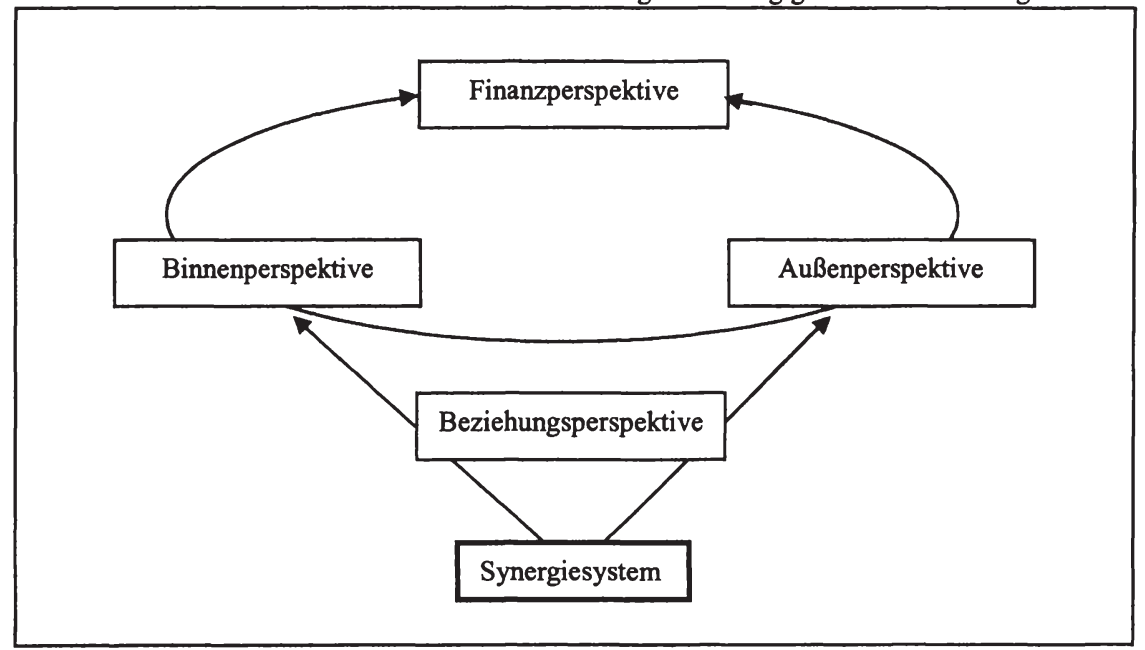

Quelle: Eigene Darstellung in Anlehnung an Kaplan/Norton 1998, 154

Um die Werte der Variablen Erfolg im Zuge der quantitativen Untersuchung erhebbar zu machen, müssen Dimensionen formuliert werden, welche den Erfolg aus der jeweiligen Perspektive erfassen. Bei der Auswahl der Dimensionen muss auf die Einschränkungen Rücksicht genommen werden, die sich aus der Methode der Fragebogenerhebung ergeben. Dies bezieht sich im Hinblick auf den zu minimierenden Aufwand für die Befragten, nicht nur auf die Anzahl der verwendeten Dimensionen, sondern auch auf deren inhaltlichen Anspruch an die Informiertheit der Befragten. Um die Qualität der Dimensionen möglichst sicher zu stellen, werden an dieser Stelle zuerst vorläufige Dimensionen formuliert, die dann in Expertengesprächen auf ihre Tauglichkeit hin untersucht werden. Durch die Zwischenschaltung dieser qualitativen Erhebung kann vor Durchführung der quantitativen Erhebung eine Brücke zum Feld geschlagen werden, die einen wesentlichen Beitrag zur Präzision der Erhebungsinstrumente leisten kann. 


\subsubsection{Dimensionen der Beziehungsperspektive}

Die Beziehungsperspektive erfasst die Qualität der Beziehung zwischen den Beteiligten eines Synergiesystems. Zur Erfassung der Beziehungsqualität werden folgende Dimensionen herangezogen:

\subsection{Kommunikationsqualität}

Mit dieser Dimension soll erfasst werden, wie die beteiligten Unternehmen miteinander kommunizieren. Es geht dabei um die Häufigkeit der Kommunikationskontakte und deren Intensität. Auch die Effizienz der Kommunikationswege und der Strukturierungsgrad der Kommunikation soll bei Erhebung der Kommunikationsqualität berücksichtigt werden. Die Qualität der Kommunikation zwischen den Beteiligten in einem Synergiesystem beeinflusst den Abgleich der Positionen und den Gedankenaustausch innerhalb des Systems und ist somit von wesentlicher Bedeutung für Koordination innerhalb des Synergiesystems. Nur eine effiziente Koordination der Interessen und des Vorgehens gewährleistet, dass die beteiligten Unternehmen im Synergiesystem ihr Know-How einbringen und ihre Interessen wahren können. ${ }^{239}$ Eine gute Koordination der Know-HowBeiträge der an einem Synergiesystem beteiligten Unternehmen trägt zum Erfolg des Synergiesystems bei. Die Qualität der Koordination der Interessen der an einem Synergiesystem Beteiligten beeinflusst zudem die Beziehungsqualität unter den Kooperateuren.

\subsection{Stabilität und Belastbarkeit - Verlässlichkeit}

Die Dimension Stabilität und Belastbarkeit umfasst all jene Aspekte des Synergiesystems, die im Allgemeinen nur in Krisensituationen in das Zentrum der Aufmerksamkeit der Akteure rücken. Wie robust (d.h. wie verlässlich) das Synergiesystem gegen Krisen ist und inwieweit ausgeprägt die Kooperateure die Konfliktlösungskompetenzen innerhalb des Synergiesystems einschätzen, wird mit dieser Dimension erfasst. Es geht hier auch um die Frage, ob die Kooperateure Probleme ansprechen und ob solche Diskussionen, so sie stattfinden, in einem konstruktiven Gesprächsklima ausgetragen werden.

\subsection{Sympathie und persönliche Beziehung}

In Unternehmen arbeiten Menschen. Ohne Menschen gibt es kein Unternehmen. Bei Kooperationen wird dieser Umstand ganz besonders deutlich. Der Erfolg 
einer Kooperation zeigt sich unter anderem auch an der Qualität der interorganisationalen Beziehungen. Dabei ist zu erfragen, ob der Ansprechpartner im Kooperationspartnerunternehmen als Repräsentant des Unternehmens dem er angehört oder als Mitglied des Synergiesystems angesehen wird. Auch die Frage, inwieweit der Ansprechpartner im Kooperationspartnerunternehmen als Funktionsträger oder als Mensch angesehen wird, wird mit dieser Dimension erfasst. Es ist auch denkbar, dass sich unter den Mitgliedern eines Synergiesystems unternehmens-übergreifende persönliche Beziehungen bis hin zu Freundschaften entwickeln. Solche Freundschaften deuten auf eine hohe Beziehungsqualität hin, denn wer sich beruflich nicht gut versteht wird sich kaum befreunden. Außerdem können im Rahmen solcher persönlicher Beziehungen Misshelligkeiten bereinigt werden, bevor sie zu Problemen anwachsen, die den Erfolg und den Bestand der Kooperationsbeziehung gefährden. ${ }^{240}$

\subsection{Transparenz und Ehrlichkeit}

Mit dieser Dimension soll erfasst werden, wie offen die Kooperationspartner miteinander umgehen. Es soll beleuchtet werden, ob der Akteur Einblick in die Vorgänge und Abläufe im Unternehmen seines Kooperationspartners hat und wie gut er sich von diesem über Vorgänge und Abläufe, die für das Synergiesystem relevant sind, informiert fühlt. Auch die Frage, inwieweit der Akteur das Gefühl hat, von seinem Kooperationspartner stets ausreichende sowie zutreffende Informationen bereitgestellt zu bekommen, ist ein wichtiger Punkt, der von dieser Dimension erfasst werden soll. Nur wenn die Kooperationspartner offen und ehrlich miteinander umgehen und sich so gegenseitig Einblick in die Vorgänge und Abläufe im jeweils fremden Unternehmen ermöglichen, kann eine effiziente Koordination der Aktivitäten im Synergiesystem gewährleistet werden. Somit stellt die Dimension Transparenz und Ehrlichkeit einen Aspekt der Kooperationsbeziehung dar, der bei der Beurteilung des Erfolgs eines Synergiesystems berücksichtigt werden muss.

\subsection{Entscheidungsfindung}

Ein wichtiges Element für eine gute Beziehung zwischen den Kooperationspartnern und für den Erfolg eines Synergiesystems ist die Qualität des Entscheidungsfindungsprozesses. Dabei ist besonders zu beachten, wie Entscheidungen zu Stande kommen. Werden Entscheidungen wirklich gemeinsam getroffen, hat also das Wort aller Kooperationspartner das gleiche Gewicht im Entscheidungsfind- 
ungsprozess innerhalb des Synergiesystems, so wird sich das positiv auf die Qualität der Beziehung der Kooperateure auswirken, da keiner der Beteiligten das Gefühl hat, übervorteilt worden zu sein. Nur bei egalitärem Einfluss auf das Ergebnis des Entscheidungsfindungsprozesses werden alle Beteiligten das Gefühl haben, von ihren Partnern ernst genommen zu werden. Dabei kommt es jedoch nicht so sehr auf den faktisch gleich großen Einfluss auf das Ergebnis des Entscheidungsfindungsprozesses an, sondern es ist vor allem die subjektive Einschätzung des eigenen Einflusses relativ zu dem der anderen Beteiligten auf den Entscheidungsfindungsprozess für die Qualität der Kooperationsbeziehung von Bedeutung. Ein zweiter wichtiger Punkt innerhalb dieser Dimension ist die Frage nach taktischem Verhalten der Kooperationspartner im Entscheidungsfindungsprozess. Verhält sich ein Beteiligter in einem Synergiesystem taktisch, so handelt er innerhalb des Synergiesystems vor dem Hintergrund von Motiven, die den Zielen der eigenen Unternehmung und nicht der Zielsetzung des Synergiesystems verpflichtet sind. Die Ziele der einzelnen Unternehmen können mit den Zielen des Synergiesystems in konfliktärem Verhältnis stehen. Verhält sich ein Kooperateur in einem Entscheidungsfindungsprozess innerhalb eines Synergiesystems taktisch, so beeinflusst er das Ergebnis dieses Prozesses derart, dass es im Sinne der Ziele seines Unternehmens verzerrt wird. So ist die Entscheidung für das Synergiesystem suboptimal, was sich auch negativ auf die anderen an diesem Synergiesystem beteiligten Unternehmen auswirkt. Taktisches Verhalten eines an einem Synergiesystem Beteiligten stellt somit eine Beeinträchtigung der Beziehungsqualität innerhalb eines Synergiesystems dar.

\subsubsection{Finanzperspektive}

Die Finanzperspektive soll jenen Aspekt des Erfolgs der Unternehmen beleuchten, der Aussagen über die aktuelle sowie die zukünftige Entwicklung der Gewinne möglich macht. Eine positive Gewinnentwicklung ist ein wesentlicher Faktor für den Erfolg des Unternehmens, denn sie ermöglicht die Leistungsfähigkeit der Organisation und ein ausreichendes Unternehmenswachstum sowie Entnahmen bzw. Gewinnausschüttungen. ${ }^{241}$

Um im Zuge der quantitativen empirischen Untersuchung eine Erfassung der relevanten Merkmale der befragten Unternehmen im Rahmen der Finanzperspektive zu gewährleisten, wird hier auf das Schema der erfolgswirtschaftlichen Analyse, als Methode der Beurteilung der zukünftigen nachhaltigen Gewinnerzielungs- 
fähigkeit von Unternehmen, zurück-gegriffen. ${ }^{242}$ Im Folgenden werden die einzelnen Kennzahlen, ihre Zusammensetzung und ihre Aussagekraft für die Erfolgsbeurteilung kurz besprochen.

\subsection{Umsatzbezogene Kennzahlen}

Eine der wichtigsten Größen bei der Unternehmensanalyse ist der Umsatz, denn dieser ist im Rahmen der Unternehmensanalyse eine der wenigen Größen, die genau definierbar sind und bei denen es keine Unterschiede bei der Berechnungsart gibt. ${ }^{243}$ Daher erscheinen die umsatzbezogenen Kennzahlen als für vorliegenden Zweck besonders geeignet.

- Umsatzwachstumsrate

$$
\text { Umsatzwachstumsrate }=\frac{\text { Umsatz des Geschäftsjahres }}{\text { Umsatz des Vorjahres }} \times 100
$$

Der Umsatz im aktuellen Bilanzjahr relativ zum Umsatz des Vorjahres wird durch die Umsatzwachstumsrate ausgedrückt. Ein positiver Wert zeigt ein Umsatzwachstum innerhalb der Betrachtungsperiode an. Analog dazu zeigt ein negativer Wert einen Umsatzrückgang in der Periode an. Da, wie oben besprochen, der Umsatz eine der wichtigsten Determinanten des Unternehmenserfolgs in finanzieller Hinsicht darstellt, enthält die Umsatzwachstumsrate für die vorliegende Untersuchung relevante Informationen über den Erfolg des Unternehmens innerhalb der Betrachtungsperiode.

- Cash-Flow-Umsatzrate

$$
\text { Cash-Flow-Umsatzrate }=\frac{\text { Cash-Flow }}{\text { Umsatz }} \times 100
$$

Die Cash-Flow-Umsatzrate zeigt an, in welchem Maß der Umsatz zum Innenfinanzierungspotential wird. Das heißt, diese Kennzahl gibt an, welcher Anteil des Umsatzes für Investitionen, Schuldentilgung und Gewinnausschüttung zur Verfügung steht. ${ }^{244}$ Als Maßzahl für das Potential der Innenfinanzierung eines Unternehmens, ist die Cash-Flow-Umsatzrate ein wichtiges Element bei der Beurteilung des Unternehmenserfolges. 


\subsection{Rentabilitätskennzahlen}

Um den Bestand eines Unternehmens auch mittel- und langfristig zu gewährleisten, ist es notwendig, dass die Ertragskraft des Unternehmens gestärkt wird oder zumindest erhalten bleibt.

„Die Rentabilität ist eine Kennzahl, welche die Ertragskraft eines Unternehmens ausdrückt [...]. Sie wird als Relativzahl, die sich aus der Inbeziehungsetzung einer Erfolgsgröße zu einer diesen Erfolg mitbestimmenden Einflussgröße ergibt, ausgedrückt.“245

Die Rentabilitätskennzahlen drücken die Verzinsung des im Unternehmen eingesetzten Kapitals aus. Diese Verzinsung stellt ein Maß für den Erfolg des Unternehmens dar. ${ }^{246}$ Die Rentabilitätskennzahl, die für vorliegenden Zweck als besonders geeignet erscheint, ist die Umsatzrentabilität. Der Grund dafür liegt einerseits in der Aussagekraft dieser Kennzahl und andererseits in dem Umstand, dass die zur Berechnung der Umsatzrentabilität benötigten Größen im Zuge der Fragebogenbefragung relativ leicht erhoben werden können.

- Umsatzrentabilität

$$
\text { Umsatzrentabilität }=\frac{\text { Jahresüberschuss bzw. Betriebserfolg }}{\text { Umsatzerlöse }} \times 100
$$

Die Umsatzrentabilität gibt die Höhe des aus dem Umsatz zur Verfügung gestellten Betrags zur Deckung des eingesetzten Kapitals an. ${ }^{247}$ Dabei bedeutet eine Umsatzrentabilität von beispielsweise 4\%, dass das Unternehmen 100 Euro Umsatz machen muss, um 4 Euro Gewinn zu erwirtschaften. ${ }^{248}$ Sinkt die Umsatzrentabilität, so bedeutet das weniger Gewinn pro umgesetztem Euro. Für Veränderungen der Umsatzrentabilität können sowohl Veränderungen der Aufwandseite als auch der Erlösseite verantwortlich gemacht werden. So führt eine Erhöhung des Aufschlagsprozentsatzes oder eine Senkung der Fixkosten zu einer Erhöhung der Umsatzrentabilität. Eine Erhöhung des Aufschlagsprozentsatzes kann beispielsweise mittels niedrigeren Einkaufspreisen, höheren Verkaufspreisen oder einer Sortimentsbereinigung und einer damit verbundenen Senkung der Fixkosten erzielt werden. Die Umsatzrentabilität ist somit eine Kennzahl, die 
Informationen darüber liefert, wie erfolgreich ein Unternehmen mit seinen Umsatzerlösen wirtschaftet.

\subsubsection{Binnenperspektive}

Diese Perspektive betrachtet den Erfolg auf der Ebene der Unternehmen in Hinblick auf das „Innenleben“ des Unternehmens. Die Binnenperspektive soll die Qualität der inneren Strukturen und Abläufe beleuchten. Dazu werden die folgenden drei Dimensionen formuliert.

\subsection{Mitarbeiterfluktuation}

Unter Mitarbeiterfluktuation werden hier jene Arbeitsplatzwechsel verstanden, die aufgrund einer autonomen Entscheidung des Mitarbeiters, in einer anderen Organisation ein Arbeitsverhältnis einzugehen, entstehen. ${ }^{249}$ Innerorganisationale ${ }^{250}$ Arbeitsplatzwechsel werden nicht berücksichtigt.

Die Mitarbeiterfluktuation hat vielfältige Auswirkungen auf den Erfolg des Unternehmens. Auf der Seite der negativen Auswirkungen stehen meist die Kosten der Ersatzeinstellung im Vordergrund. Die Kosten der Ersatzeinstellung ergeben sich einerseits aus den Anwerbungskosten, die sich ihrerseits wiederum aus den Rekrutierungskosten, den Auswahlkosten und den Anstellungs- und Platzierungskosten zusammensetzen. ${ }^{251}$ Zusätzlich fallen bei der Ersatzeinstellung noch Lernkosten ${ }^{252}$ an. Auch die Kosten die dadurch entstehen, dass der ausscheidende Mitarbeiter gegen Ende seines Beschäftigungsverhältnisses nicht mehr die volle Leistung erbringt ${ }^{253}$ und die Kosten die durch die Vakanz der Stelle während der Ersatzsuche anfallen ${ }^{254}$, werden unter dem Begriff Austrittskosten diskutiert.

Aber auch für die Sozialstruktur innerhalb des Unternehmens hat das Ausscheiden eines Mitarbeiters Auswirkungen. So ist es denkbar, dass der ausgeschiedene Mit-

249 vgl Kremsner 1983, 36

250 Der Wechsel eines Mitarbeiters von einer Abteilung in eine andere Abteilung desselben Unternehmens. (vgl. Sabathil 1977, 14)

$251 \mathrm{vgl} \mathrm{Mobley/Hand/Becker/Meglino} 17$

252 Lernkosten sind beispielsweise Kosten der Ausbildung („training on the job") und Einarbeitung. Sie richten sich in ihrer Höhe danach, wie stark sich das persönliche Qualifikationsprofil mit dem Anforderungsprofil der Stelle decken.

253 Schätzungen dieses Effekts liegen zwischen 25\% und 75\% der Arbeitszeit! (vgl. Stenz 1973, 107)

254 Solche Kosten entstehen beispielsweise durch einen Produktionsausfall, der auf die Abwesenheit des ausgeschiedenen Mitarbeiters zurückzuführen ist oder durch die Umverteilung der Arbeit auf die verbliebenen Mitarbeiter. 
arbeiter in der Organisation eine spezielle Rolle in der informellen Struktur inne hatte. Scheidet nun ein Mitarbeiter aus der Organisation aus, der beispielsweise die informelle Rolle eines ,institutionalisierten Problemlösers“ ausfüllte, so fehlt der Organisation nunmehr diese Kompetenz. Abgänge einflussreicher Mitarbeiter können Machtkämpfe nach sich ziehen. All diese Erschütterungen der Sozialstruktur des Unternehmens, die durch Mitarbeiterfluktuation ausgelöst werden (können), schränken die Funktionsfähigkeit der Organisation ein und binden Ressourcen.

Eine weitere negative Auswirkung der Mitarbeiterfluktuation ist der Verlust an Humankapital. Ein Arbeitsplatzwechsel wird für das Individuum nur dann in Frage kommen, wenn der neue Arbeitsplatz attraktiver ist als der bisherige. Unter der plausiblen Annahme, dass die Attraktivität eines Arbeitsplatzes positiv mit der Leistungsfähigkeit des Arbeitnehmers korreliert ${ }^{255}$, ergibt sich eine Situation, in der tendenziell fähige Mitarbeiter aus der Organisation ausscheiden um in einer anderen Organisation einen Arbeitsplatz anzunehmen. Der Verlust dieser „High Performers" bleibt nicht ohne Wirkung auf den Unternehmenserfolg.

In der Literatur sind auch Argumente für positive Auswirkungen der Mitarbeiterfluktuation auf das Unternehmen zu finden. So wird die positive Wirkung neuer Ideen, die in den Köpfen neuer Mitarbeiter durch die Fluktuation in das Unternehmen getragen werden, ins Treffen geführt. Gleichzeitig wird dieses Argument aber insofern relativiert, als die neuen Mitarbeiter sich erst einarbeiten müssen und sich Akzeptanz innerhalb der neuen Organisation schaffen müssen, ehe sie Veränderungen durchsetzen können. Es ist anzunehmen, dass dieser Sozialisationsprozess einen Großteil der Innovationskraft der neuen Köpfe kostet und sich der positive Effekt der Mitarbeiterfluktuation auf die Innovationskraft des Unternehmens in Grenzen hält.

Ähnliches gilt für das Argument, wonach die Änderung der Zusammensetzung des Mitarbeiterstammes durch Mitarbeiterfluktuation zu einer gesteigerten Anpassungsfähigkeit des Unternehmens an sich ständig ändernde Umweltbedingungen führt. Auch da ist festzuhalten, dass sich tendenziell eher der Mitarbeiter an das Unternehmen anpassen wird, als das umgekehrt der Fall ist. Aber nur wenn sich das Unternehmen an den neuen Mitarbeiter anpassen würde, käme es durch Mitar-

255 Diesem Argument liegt die Annahme zugrunde, dass die Arbeitsplätze, die einem Arbeitnehmer auf einer gewissen Qualifikationsstufe angeboten werden, innerhalb einer gewissen Attraktivitätsbandbreite liegen, die sich durch unterschiedliche Anreiz-Beitrags-Relationen ergibt. 
beiterfluktuation zu einer höheren Anpassungsfähigkeit des Unternehmens. Daher wird auch dieser Effekt als eher gering eingeschätzt.

Als völlig verfehlt kann der angeblich positive Effekt der Mitarbeiterfluktuation auf das Unternehmen durch Konfliktreduktion angesehen werden. Es wird behauptet, dass der Arbeitsplatzwechsel in Konfliktsituationen als Konfliktlösungsstrategie zur Konfliktreduzierung führt, was wiederum die Spannungen im Unternehmen reduziert und der Funktionsfähigkeit der Organisation förderlich ist. Ist der Gewinn einer erfolgreichen Konfliktbewältigung nicht gerade die gewonnene Konfliktlösungskompetenz aller Beteiligten? Scheidet ein Beteiligter aus und löst somit einen bestehenden Konflikt, so hat das keinerlei Auswirkung auf die Konfliktlösungskompetenz der Beteiligten. Das Unternehmen hat einen Mitarbeiter verloren und nichts gewonnen, denn in einer ähnlichen Konfliktkonstellation ist die Organisation wieder zu keiner Konfliktbewältigung fähig. Sie hat gelernt, dass sich das Problem von selbst löst: durch Fluktuation.

In Summe kann somit von einem deutlichen Überhang der negativen Effekte der Mitarbeiterfluktuation auf den Erfolg des Unternehmens ausgegangen werden.

\subsection{Verlässlichkeit der Prognosen}

Prognosen sind der Versuch eine sich mehr oder weniger abzeichnende Zukunft zu ergründen und einen zukünftigen Zustand oder Wert vorauszusagen. Dabei basiert jede Prognose auf unvollständiger Information und ist deshalb ungenau. ${ }^{256}$ Prognosen sind mit Unsicherheit behaftet, die ihre Verlässlichkeit betrifft. Die Zukunft kann das bringen, was die Prognose vorausgesagt hat, oder eben nicht. Beide Möglichkeiten sind mit gewissen Wahrscheinlichkeiten ihres Eintretens verknüpft. Die Eintrittswahrscheinlichkeit eines Zukunftsszenarios, das von einer absolut verlässlichen Prognose gezeichnet wird, beträgt 1. Tatsächlich werden die Eintrittswahrscheinlichkeiten kleiner 1 sein. Der Wert der Eintrittswahrscheinlichkeit, also der Zuverlässigkeit der Prognose ist abhängig vom Vollständigkeitsgrad der qualitativen sowie quantitativen Information über die Wirkungszusammenhänge, die in dem Bereich, für den die Prognose erstellt wird, relevant sind. Die Qualität der Prognosen ist für den Erfolg des Unternehmens von großer Bedeutung, da auf ihrer Basis weitreichende Entscheidungen getroffen werden, die Ressourcen des Unternehmens auf ein ganz bestimmtes, nämlich prognostiziertes, Zukunftsszenario fokussieren. 
Nun ist die Informationsbasis einerseits schon abhängig von den gegebenen Faktoren der Umwelt des Unternehmers, sie kann jedoch andererseits von diesem sehr wohl durch aktives Informationsmanagement verbessert werden. Die Dimension „Qualität der Prognosen“ beleuchtet die Qualität des Informationsmanagements innerhalb der Unternehmen. Die Zuverlässigkeit der in einem Unternehmen aufgestellten Prognosen ist ein Hinweis darauf, wie erfolgreich das Unternehmen relevante Informationen einholt und für sich nutzen kann und stellt daher eine berechtigte Dimension zur Beurteilung des Unternehmenserfolges dar.

\subsubsection{Außenperspektive}

Dieser Blickwinkel versucht den Erfolg des Unternehmens im Außenverhältnis zu erfassen. Auf der einen Seite wird untersucht, wie erfolgreich das Unternehmen seine Kunden zufrieden stellt, zu wiederholten Kauftransaktionen animieren kann und neue Kunden akquiriert. Die Kundenperspektive beleuchtet somit quasi den Erfolg des Unternehmens ,im Kopf des Kunden“. Auf der anderen Seite wird die Beziehung des Unternehmens zu seinen Lieferanten zur Beurteilung des Erfolges herangezogen. Zur Erfassung dieses Aspektes des Unternehmenserfolges werden bei der Erhebung folgende Dimensionen abgefragt.

\subsection{Kundenzufriedenheit und Stammkundenanteil}

Simon/Homburg stellen fest: „Die Kundenzufriedenheit ist ein Mittel zur Stei-

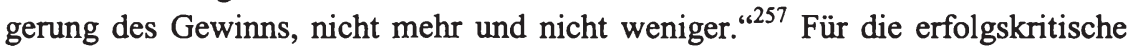
Rolle der Kundenzufriedenheit werden vor allem zwei Gründe angegeben: Einerseits kostet die Akquirierung eines neuen Kunden vier bis sechs mal soviel wie die Bindung eines Altkunden ${ }^{258}$ und andererseits konsumieren Altkunden im Durchschnitt mehr als Neukunden. ${ }^{259}$ Die hohen Akquisitionskosten und die niedrige durchschnittliche Konsumation neuer Kunden führen dazu, dass ein neuer Kunde bis zum vierten Jahr keinen Gewinn für das Unternehmen erzeugt. ${ }^{260}$

257 Simon/Homburg 1995, 26 siehe dazu auch Quartapelle/Larsen 1996, 94

258 Müller/Riesenbeck 1991, 69 sowie Bruhn 1996, 7

„Eine Erhöhung der Kundenzufriedenheit um jeweils einen Indexpunkt [des schwedischen Kundenbarometers] über fünf Jahre bedeutet eine durchschnittliche Steigerung des ROI (Return on Investment) von 11,33\%." (Mayer/Dornach 1995, 167)

Eine branchenübergreifende Analyse der Wirkung der Kundenbindung auf den Gewinn, zeigt, dass - in Abhängigkeit von der Branche - der ROI über die gesamte durchschnittliche Kundenbindungsdauer um 25 bis 85 Prozent gesteigert werden konnte, wenn die Kundenabwanderungsrate in den untersuchten Unternehmen um 5 Prozent gesenkt wurde. (vgl. Reichelt/Sasser 1991 zitiert in: Lötschner 1998, 4)

259 Quartapelle/Larsen 1996, 95 sowie Töpfer 1996, 1

260 Quartapelle/Larsen 1996, 94 
Außerdem sind Stammkunden loyaler als Neukunden. Untersuchungen haben gezeigt, dass Stammkunden weniger preisempfindlich sind als Neukunden. ${ }^{261}$ Auch die Auswirkungen der Kundenzufriedenheit auf das Unternehmensimage durch Mundpropaganda darf nicht unterschätzt werden. ${ }^{262}$ Ein Anteil von $75 \%$ zufriedener Kunden kann einen zwanzigprozentigen Anteil von unzufriedenen Kunden nicht ausgleichen und das Unternehmensimage wird unvorteilhaft ausfallen. $^{263}$

Es zeigt sich, dass zufriedene Kunden zu Stammkunden werden ${ }^{264}$, die für den Erfolg des Unternehmens von großer Bedeutung sind. ${ }^{265}$ Somit stellen der Stammkundenanteil und die Kundenzufriedenheit geeignete Dimensionen dar, um den Unternehmenserfolg aus der Kundeperspektive zu beleuchten.

\subsection{Marktanteilsentwicklung}

Der Marktanteil ist der prozentmäßige Anteil des Absatzvolumens des Unternehmens am gesamten Marktvolumen. ${ }^{266}$ Die Bewertung des Absatzvolumens und des Marktvolumens erfolgt hier wertmäßig und nicht mengenmäßig. Das bedeutet, dass der Wert der abgesetzten Leistungseinheiten des Unternehmens mit dem Wert der abgesetzten Leistungseinheiten auf dem Gesamtmarkt in Relation gesetzt wird.

Die Heranziehung der Marktanteilsentwicklung für die Erhebung des Unternehmenserfolgs aus der Außenpektive wird durch zwei Konzepte gerechtfertigt, die einen erfolgswirksamen Zusammenhang zwischen dem Marktanteil und der Kostensituation eines Unternehmens herstellen. Beide, sowohl die „Economies of Scale" als auch das Konzept der Erfahrungskurve, stellen auf ein Sinken der Durchschnittskosten bei steigender Ausbringungsmenge ab. ${ }^{267}$

„Für den kumulierten Gewinn wird der sogenannte „break-even point“ sogar erst nach sieben Jahren erreicht.“ (Quartapelle/Larsen 1996, 94) Der ,break-even point" ist in diesem Zusammenhang jener Zeitpunkt zu dem die mit einem Neukunden in Zusammenhang stehenden Kosten, durch die Einnahmen durch seinen Konsum gedeckt werden.

261 Homburg/Rudolph 1995, 43

262 „Alle Marktforschungsstudien [zu diesem Thema] zeigen, dass zwischen 50 und $70 \%$ aller Käufe auf Empfehlungen von Freunden zurückzuführen sind." (Horovitz/Panak 1993, 95)

263 Töpfer/Mann 1996, 58

264 Quartapelle/Larsen 1996, 94f sowie Meyer/Dornach 1998, 184

265 siehe dazu Anderson/Sullivan 1993 oder Anderson/Sullivan/Lehmann 1994

266 Fuchs 1977, 650

267 Buzell/Gale/Sultan 1975, 98 
„Economies of Scale“ sind die Bezeichnung für das Resultat einer Kostendegression, die sich durch einen relativen Rückgang der Gesamtkosen und einen absoluten Rückgang der Kosten pro Outputeinheit bei steigender Produktionsmenge ergibt. ${ }^{268}$ Die Kostendegression wird auf drei Effekte zurückgeführt: Erstens kommt es bei einem Anstieg der Outputmenge zur Größendegression. Dieser Ausdruck bezeichnet den Teil der Kostendegression bei Outputerhöhung, der durch eine Annäherung an die optimale Unternehmensgröße zu Stande kommt. Zweitens kommt es durch eine Steigerung des Kapazitätsausnutzungsgrades zu einer Kostendegression, die als Kapazitätsausnutzungsdegression bezeichnet wird. Der dritte Effekt eines Outputanstieges auf die Gesamt- und die Stückkosten ist die Auflagendegression. Dabei handelt es sich um eine Kostendegression deren Ursache eine Erhöhung der Stückzahl gleichartiger Outputeinheiten (Auflage) ist. $^{269}$

Über diese Wirkungszusammenhänge hat die Outputmenge Einfluss auf die Kostenstruktur des Unternehmens. Eine Erhöhung des Marktanteils hat per Definition eine Erhöhung der Outputmenge zur Folge. Somit ist der Zusammenhang zwischen Marktanteil und Unternehmenserfolg hergestellt: Unternehmen mit hohem Marktanteil profitieren, auf Grund ihrer höheren Ausbringungsmenge, viel mehr von degressiven Kostenverläufen als Unternehmen mit geringem Marktanteil. ${ }^{270}$

Das zweite Konzept, das einen Zusammenhang zwischen den Durchschnittskosten und der Ausbringungsmenge herstellt, ist die Erfahrungskurve. Dieses Konzept sagt einen Rückgang der gesamten Durchschnittskosten der Produktion und Distribution eines Gutes um zwanzig bis dreißig Prozent bei Verdoppelung der kumulierten Outputmenge des Unternehmens voraus. ${ }^{271}$ Bei diesem Konzept wird die Kostenreduktion auf folgende Faktoren zurückgeführt: Übungsgewinne reduzieren die Fertigungszeiten und damit auch die Fertigungskosten pro Stück. ${ }^{272}$ Durch den Einsatz besserer Technologien und Produktionstechniken sowie durch fortschreitende Arbeitsteilung und Spezialisierung können die Stückkosten gesenkt werden. Außerdem kann bei einer höheren Ausbringungsmenge durch die Entwicklung und die Verwendung produktionskostengünstiger Produktdesigns

268 Mellerowicz 1973, 319

269 vgl. Mellerowicz 1973, 319

270 vgl. Neubauer 1980, 143

$271 \mathrm{vgl}$. Buzell/Gale/Sultan 1975, 98

272 Classen 1984, 50 
mehr an Kosten eingespart werden, als das bei einer niedrigeren Ausbringungsmenge der Fall ist. ${ }^{273}$

Sofern im Unternehmen die potentiellen Kostenrückgänge durch das Management realisiert werden, ergibt sich aus dem Konzept der Erfahrungskurve ein umgekehrt proportionales Verhältnis zwischen Kosten und Marktanteil, ${ }^{274}$ was weiter einen positiven Einfluss eines Marktanteilswachstums auf den Erfolg des Unternehmens bedeutet.

Abgesehen von den Konzepten der „Economies of Scale" und der Erfahrungskurve bringt ein hoher Marktanteil auch Vorteile am Beschaffungsmarkt sowie am Absatzmarkt. Die günstigere Situation am Beschaffungsmarkt für Unternehmen mit einem hohen Marktanteil ergibt sich als Folge von Umsatz- und Mengenrabatten und geringeren Transportstückkosten. Am Absatzmarkt kann das Unternehmen mit großem Marktanteil seine überlegene Kostensituation in Form von attraktiveren Preisen geltend machen und so Preisdruck auf seine Konkurrenten ausüben oder es schöpft eine höhere Gewinnspanne ab.

All diese Effekte sind im Bereich der Klein- und Mittelbetriebe, auf die sich die vorliegende Untersuchung konzentriert, nur vor dem Hintergrund eines Vergleichs der relativen Vorteilhaftigkeit der Situation der in einem Markt tätigen kleinen und mittleren Unternehmen zu verstehen. Obschon darauf hinzuweisen ist, dass gerade die kleinen und mittleren Unternehmen häufig in Nischenmärkten tätig sind, in denen sie dann sehr wohl beträchtliche Marktanteile erzielen.

\subsection{Anteil der Stammlieferanten}

Legt ein Zulieferer ein Angebot, so legt er sich auf das von ihm Angebotene fest. Diese Festlegung birgt für ihn ein Risiko, da sie von Seiten des Abnehmers ausgenützt werden kann. ${ }^{275}$ Je länger der Zulieferer seinen Abnehmer kennt und um so besser das Klima der Geschäftsbeziehung ist, umso geringer wird seine Risikoeinschätzung für Transaktionen mit diesem Abnehmer ausfallen. Einem Risiko ist immer ein Risikoäquivalent entgegenzustellen. Im Falle einer Zulieferung kann ein erhöhtes Risiko des Zulieferers - bedingt durch ein schlechtes Beziehungsklima - beispielsweise über einen Preiszuschlag, weniger Flexibilität, schlechtere

273 Hedley 1977,98

274 BCG 1970, 1ff

275 Übliche Einkäufertricks sind beispielsweise: Das Arbeiten mit überhöhten Stückzahlen, das Versenden von Eilanfragen, das Berufen auf ehemalige Mitarbeiter des Zulieferers usw. (vgl. Riffner/Weidelich 2001, 142f) 
Lieferbedingungen oder weniger vorteilhafte Zahlungsbedingungen ausgeglichen werden. ${ }^{276}$ So bringt eine langjährige gute Geschäftsbeziehung zwischen Zulieferer und Abnehmer, über eine niedrige Risikoeinschätzung des Zulieferers, erfolgswirksame Vorteile für den Abnehmer und wirkt sich so positiv auf dessen Unternehmenserfolg aus. ${ }^{277}$

\subsection{Angebots-Zuschlagsrate}

Wird ein Angebot über einen Auftrag zur Leistungserstellung gelegt, so kommt es zu einem direkten Vergleich der Leistungsfähigkeit des Unternehmens mit der Leistungsfähigkeit der im selben Markt tätigen Konkurrenzunternehmen. Den Zuschlag bekommt jenes Unternehmen, dessen Angebot für den Auftraggeber am vorteilhaftesten ist. Die Attraktivität eines Angebots ergibt sich aus dem Verhältnis zwischen der angebotenen Leistung und dem Preis. Hat ein Unternehmen in dieser Konkurrenzsituation eine hohe Erfolgsquote, so ist das Verhältnis zwischen der Leistung, die es bietet und dem Preis, den es für seine Leistungen verlangt, für den Auftraggeber attraktiver als die Angebote der Konkurrenzunternehmen. Dies kann auf zweierlei Weise zustande kommen: Entweder das Unternehmen ist in der Lage für die gleiche Leistung zu niedrigeren Kosten als die Konkurrenzunternehmen bereitzustellen, was ihm ermöglicht, einen niedrigeren Preis zu verlangen, oder es kann zu gleichem Preis mehr Leistung anbieten. Beides deutet auf eine vergleichsweise gute Gestaltung der inneren Strukturen und Abläufe im Unternehmen hin. Nur Unternehmen, die die inneren Strukturen, welche die Leistungserstellung ermöglichen und die Abläufe, welche zur Leistungserstellung führen, erfolgreich optimieren, können attraktive Angebote legen, für die sie sodann den Zuschlag erhalten. Somit ist die AngebotsZuschlags-Rate eine Maßzahl für die Qualität der inneren Strukturen und der Abläufe in einem Unternehmen.

\subsubsection{ZUSAMMENFASSUNG: DIE VORLÄUfIGEN DIMENSIONEN DER VARIABLEN}

Aus den bisherigen Ausführungen ergeben sich für die drei latenten Variablen, deren Ausprägungen in der folgenden empirischen Untersuchung erhoben werden

276 Riffner/Weidelich geben einen zusammenfassenden Erlebnisbericht: „Das - wenn auch nicht immer einheitliche - Ergebnis der Diskussion besagt, dass ein wenig Pokern im Sinne des Einkäufers sein darf. Dass sich aber ein gezieltes und zur Tagesordnung gehörendes Verhalten wie oben erwähnt bei den Lieferanten herumspricht, dem Image des Unternehmens schadet und sich das Verhalten der Verkäufer in wirklichen Notfällen in Form von hohen Kosten rächt, dürfte allerdings einleuchten." Riffner/Weidelich 2001, 152

277 vgl. Riffner/Weidelich 2001, 143 
sollen, die in Abbildung 16 dargestellten Dimensionen. Diese Dimensionen werden nun im Zuge einer qualitativen Erhebung auf ihre Brauchbarkeit für die folgende großzahlige Untersuchung getestet.

Abb. 16: Die vorläufigen Dimensionen der Variablen - Ein Überblick

\begin{tabular}{|c|c|c|}
\hline $\begin{array}{c}\text { Kategorisierungsvariable } \\
\text { „Synergiesystem“ }\end{array}$ & $\begin{array}{l}\text { Erklärende Variable „Selbst- } \\
\text { verpflichtung“ }\end{array}$ & $\begin{array}{c}\text { Abhängige Variable } \\
\text { „Erfolg“ }\end{array}$ \\
\hline $\begin{array}{l}\text { Dimension aufgrund der } \\
\text { Definition der } \\
\text { Untersuchungseinheiten: } \\
\text { - Beziehung zwischen } \\
\text { Unternehmen } \\
\text { Dimensionen Kooperation: } \\
\text { Transaktionsperspektive: } \\
\text { - Selbständigkeit } \\
\text { - Freiwilligkeit } \\
\text { - Abgestimmtes Verhalten } \\
\text { - Organisierte Beziehung } \\
\text { Zeitperspektive: } \\
\text { - Nicht kurzfristige } \\
\quad \text { Perspektive } \\
\text { Dimensionen Synergiesystem: } \\
\text { Koordinationsperspektive: } \\
\text { - Gleichberechtigung } \\
\text { Möglichkeit der } \\
\text { einseitigen Defektion und } \\
\text { Systemgefährdung } \\
\text { Flexibilität }\end{array}$ & $\begin{array}{ll}\text { Vorgelagerte Dimensionen: } \\
\text { - } & \text { Erfahrung } \\
\text { - } & \text { Vertrautheit } \\
\text { - Individuelle Reputation } \\
\text { - Kollektive Reputation } \\
\text { - Perzepierte Geschichte } \\
\text { - Risikobereitschaft } \\
\text { - Selbstvertrauen } \\
\text { Nachgelagerte Dimension: } \\
\text { - Unaufgeforderte } \\
\text { Erhöhung der eigenen } \\
\text { Gefahr }\end{array}$ & $\begin{array}{l}\text { Auf Ebene des Synergie- } \\
\text { systems: } \\
\text { Beziehungsperspektive: } \\
\text { - } \quad \text { Kommunikationsqualität } \\
\text { - } \quad \text { Stabilität und Belastbarkeit } \\
\text { - Verlässlichkeit } \\
\text { - Sympathie und persönliche } \\
\text { Beziehung } \\
\text { Transparenz und Ehrlich- } \\
\text { keit } \\
\text { - Entscheidungsfindung } \\
\text { Auf Unternehmensebene: } \\
\text { Binnenperspektive } \\
\text { - Mitarbeiterfluktuation } \\
\text { - Zuverlässigkeit der Prog- } \\
\text { nosen } \\
\text { Finanzperspektive } \\
\text { - Cash-Flow-Umsatzrate } \\
\text { - Umsatzwachstumsrate } \\
\text { - Umsatzrentabilität } \\
\text { Außenperspektive } \\
\text { - } \quad \text { Kundenzufriedenheit } \\
\text { - } \quad \text { Mammkundenanteil } \\
\text { - } \quad \text { Angebotanteilsentwicklung } \\
\text { Auschlagsrate }\end{array}$ \\
\hline
\end{tabular}




\subsection{Qualitative Erhebung}

Im Rahmen der qualitativen Erhebung wurden sechs Gespräche mit Experten geführt. Das Ziel der qualitativen Erhebung war es, die im vorigen Abschnitt entwickelten Dimensionen auf ihre praktische Relevanz, ihre Erhebbarkeit im Rahmen einer postalischen Fragebogenbefragung und ihre Besonderheiten bei der technischen Durchführung zu prüfen.

In der folgenden Tabelle werden den befragten Personen jene Kompetenzfelder zugeordnet, welche von ihrer Expertise abgedeckt wurden. Die Gesprächspartner wurden so ausgewählt, dass jedes Kompetenzfeld zumindest von zwei Experten abgedeckt wurde. So konnten die Ergebnisse der einzelnen Gespräche durch wechselseitigen Abgleich auf Widersprüche kontrolliert werden. Vor dem Hintergrund der angewandten Erhebungstechnik (postalische Fragebogenbefragung) lag der Fokus der qualitativen Untersuchung auf folgenden drei Kompetenzfeldern:

1. Kompetenzfeld praktische Relevanz: Dieser Kompetenzbereich umfasst alle Fragen, die mit der Einschätzung der praktischen Relevanz der verwendeten Dimensionen in Zusammenhang stehen.

2. Kompetenzfeld Erhebbarkeit: Bei diesem Kompetenzfeld geht es um die Frage, inwieweit die Ausprägungen der jeweiligen Dimensionen im Zuge einer Fragebogenerhebung erhebbar sind. Dabei war auf die besonderen Restriktionen der postalischen Fragebogenerhebung Bezug zu nehmen.

3. Kompetenzfeld technische Durchführung: Im Zentrum dieses Themenkomplexes standen Fragen der technischen Durchfuihrung der Fragebogenerhebung. Besonderes Augenmerk wurde dabei auf die Erfolgsfaktoren einer postalischen Befragung mittels Fragebogens in Bezug auf Fragebogengestaltung, Frageformulierung, Fragen-sequenzierung, verwendete Skalen und Inhalt des Motivationsschreibens gelegt.

Die Expertengespräche wurden nach einem fünf-stufigen Schema strukturiert. Nach einer allgemeinen Diskussion des Themenkomplexes „Kooperation und Vertrauen", die den individuellen Themenzugang des Befragten erschloss (Stufe 1), wurde den Gesprächspartnern eine Tabelle (siehe Abbildung 16) vorgelegt, die alle vorläufigen Dimensionen, zugeordnet zu den interessierenden Variablen, zusammenfasst. 
Abb. 17: Zuordnung der Kompetenzfelder zu den Gesprächspartnern

\begin{tabular}{|c|c|c|c|c|}
\hline \multirow[b]{2}{*}{$\begin{array}{l}\text { Gesprächs- } \\
\text { partner }\end{array}$} & \multirow[b]{2}{*}{ Funktion } & \multicolumn{3}{|c|}{ Kompetenzfeld } \\
\hline & & Erhebbarkeit & $\begin{array}{c}\text { praktische } \\
\text { Relevanz }\end{array}$ & $\begin{array}{c}\text { technische } \\
\text { Durchführung }\end{array}$ \\
\hline $\begin{array}{l}\text { DI. Gerald } \\
\text { Sattler }\end{array}$ & $\begin{array}{l}\text { Clustermanager des } \\
\text { „Holzclusters“ Nieder- } \\
\text { österreichs }\end{array}$ & & & \\
\hline $\begin{array}{l}\text { Dr. Elisabeth } \\
\text { Steyerer-Fraut }\end{array}$ & $\begin{array}{l}\text { Clustermanagerin des } \\
\text { Wellbeeingclusters } \\
\text { Niederösterreich }\end{array}$ & & & \\
\hline $\begin{array}{l}\text { Manfred } \\
\text { Mayerhofer }\end{array}$ & $\begin{array}{l}\text { Geschäftsführender } \\
\text { Gesellschafter der } \\
\text { Bebeflex GmbH }\end{array}$ & & & \\
\hline $\begin{array}{l}\text { Dkfm. Detlev } \\
\text { Turgel } \\
\end{array}$ & $\begin{array}{l}\text { Finanzchef a.D. bei } \\
\text { IVECO Austria }\end{array}$ & & & \\
\hline $\begin{array}{l}\text { Dr. Angelika } \\
\text { Wroblewski }\end{array}$ & $\begin{array}{l}\text { Academic staff am } \\
\text { Institut für Höhere } \\
\text { Studien }\left(\mathrm{IHS}^{278}\right) \text { Wien }\end{array}$ & & & \\
\hline $\begin{array}{l}\text { Mag. Martin } \\
\text { Unger }\end{array}$ & $\begin{array}{l}\text { Academic staff am } \\
\text { Institut für Höhere } \\
\text { Studien (IHS) Wien }\end{array}$ & & & \\
\hline
\end{tabular}

Quelle: Eigene Darstellung

In den Gesprächen mit den Praktikern wurde nun jede der aufgelisteten Dimensionen auf ihre praktische Relevanz geprüft (Stufe 2). Einige Dimensionen wurden im Zuge dieser Prüfung jedoch aufgrund mangelnder praktischer Relevanz verworfen bzw. adaptiert. Dieser Schritt entfiel bei den Gesprächen mit den Experten des IHS aufgrund fehlender Expertise der Befragten in diesem Kompetenzfeld. Die praktische Relevanz der vorläufigen Dimensionen wurde für den weiteren Verlauf der Gespräche unterstellt. Dies war zulässig, da die Gespräche mit den Experten des IHS zeitlich nach den Gesprächen mit den Praktikern durchgeführt wurden. Als dritter Gesprächsblock wurde mit den Gesprächspartnern erörtert, ob es zu erwarten sei, dass den Empfängern der Fragebögen die abgefragten Informationen zur Verfügung stehen und ob die Befragten bereit sein werden, diese Informationen preiszugeben. Weiters wurde in diesem Teil der Gespräche auch über die Verständlichkeit und Eindeutigkeit der verwendeten Begriffe gesprochen (Stufe 3). Als Resultat der Gespräche mussten einige Dimensionen adaptiert werden. Anschließend wurden mit den Experten des 
IHS Fragen zur technischen Durchführung der Fragebogenbefragung diskutiert. Dabei ging es vor allem um die Optimierung des Fragebogendesigns, der Fragensequenzzierung sowie der Formulierung der einzelnen Fragen und des Motivationsschreibens (Schritt 4). Dieser Schritt entfiel bei den Gesprächen mit den Gesprächspartnern, die das Kompetenzfeld „technische Durchführung“ nicht abdeckten. Den Abschluss aller sechs Expertengespräche bildete ein Brainstorming zu bisher nicht einbezogenen Dimensionen der interessierenden Variablen, deren Integration die Erhebung der Ausprägungen der interessierenden Variablen jedoch vervollständigen würde (Schritt 5). Hier konnten in den Gesprächen einige zusätzliche Dimensionen identifiziert werden. Andere Dimensionen konnten, basierend auf den Ergebnissen der qualitativen Erhebung, für die Fragebogenerhebung adaptiert werden.

Im Folgenden werden die verworfenen, die adaptierten sowie die zusätzlichen Dimensionen dargestellt und die Argumente, die zu deren Verwerfung, Adaption oder Integration geführt haben, diskutiert.

\subsubsection{VERWORFENE DIMENSIONEN}

Als verworfene Dimensionen werden solche Dimensionen der interessierenden Variablen bezeichnet, die auf der Basis theoretischer Überlegungen zwar einen berechtigten Anspruch auf Integration in die quantitative Erhebung hätten, bei denen aber wichtige praktische Gründe einer solchen Einbeziehung in die Fragebogenbefragung entgegenstehen. Die konkreten Argumente für den Ausschluss der jeweiligen Dimension werden in den folgenden Unterpunkten dargestellt.

\subsubsection{Verworfene Dimension „Umsatzrentabilität“}

Bei der Beurteilung des Erfolgs eines Unternehmens gehören Rentabilitätskennzahlen zu den aussagekräftigsten Kennzahlen. Diese Eigenschaft würde einerseits die Einbeziehung der Kennzahl „Umsatzrentabilität“ als Dimension der Variablen Erfolg im Rahmen der Fragebogenbefragung als besonders wichtig erscheinen lassen, andererseits wurde die Bereitschaft der Empfänger der Fragebögen (Geschäftsführer kleiner und mittlerer Unternehmen), eine solchen Frage zu beantworten, von den befragten Experten als äußerst gering eingeschätzt. Besonders die befragten Praktiker wiesen darauf hin, dass die Kennzahl „Umsatzrentabilität“ zu vertraulich sei, um im Rahmen einer Fragebogenbefragung erhoben werden zu können. Diese Einschätzung konnten die Experten des IHS auf der Basis ihrer Erfahrung bestätigen. Auf Grund der befürchteten niedrigen Antwortwahrscheinlichkeit, wurde die Dimension "Umsatzrentabilität" verworfen, zumal die Ge- 
sprächspartner im Zuge der Expertengespräche die Befürchtung artikulierten, dass eine Frage nach einer so aussagekräftigen Kennzahl die Befragten skeptisch machen und so negative Auswirkungen auf die Rücklaufquote haben könnte.

\subsubsection{ADAPTIERTE DIMENSIONEN}

Bei den adaptierten Dimensionen handelt es sich um Aspekte einer interessierenden Variablen, die zwar nicht in der Form in die quantitative Erhebung einbezogen werden konnten, in der sie auf der Basis der theoretischen Überlegungen ${ }^{279}$ einbezogen werden sollten, die aber in einer adaptierten Form sehr wohl in die Fragebogenerhebung Eingang gefunden haben. Es sind dabei zwei Fälle zu unterscheiden: In dem einen Fall wurde die abzufragende Zieldimension beibehalten und nur die Ratio-Antwortskala durch eine Intervallskala ersetzt ${ }^{280}$. Als Konsequenz können nur Tendenzen nicht aber Beträge abgefragt werden. Im anderen Fall wurden Dimensionen in ihrem Bedeutungsumfang eingeschränkt oder ergänzt.

\subsubsection{Adaptierte Dimension ,langfristige Perspektive“}

Ein Kennzeichen eines Synergiesystems ist es, dass die Kooperationspartner ihr Verhalten innerhalb der Kooperation nicht an kurzfristigen Vorteilskalkülen orientieren. Das ist ein wesentlicher Faktor für das Zustandekommen hochgradig komplexer Transaktionsbeziehungen, bei denen kein kurzfristiger Erfolg für die beteiligten Unternehmen zu erwarten ist, denn nur so kann die, in einer solchen Situation bestehende, doppelte Kontingenz durchbrochen werden. Auf der Basis dieser theoretischen Überlegungen wurde die vorläufige Dimension „langfristige Perspektive“ formuliert. Im Zuge der Expertengespräche wurde jedoch klar, dass für die Durchbrechung der doppelten Kontingenz keine langfristige Erfolgsorientierung notwendig ist, sondern nur eine kurzfristige Erfolgsorientierung die Bewältigung einer solchen Situation verhindert. Daher wurde der Bedeutungsumfang erweitert und als endgültige Dimension „nicht kurzfristige Perspektive“ formuliert.

\subsubsection{Adaptierte Dimension „Flexibilität“}

Bei dieser Dimension kam es durch die Bezeichnung bei den Gesprächspartnern im Rahmen der Expertengespräche zu Missverständnissen. Die Flexibilität der Kooperationspartner wurde als mangelnder Wille zu einem klaren Bekenntnis

279 siehe dazu Abschnitt 3. Erkenntniszusammenhang 280 Zur verwendeten Skala siehe 5.3.6. Antwortkategorien, Messskala und Skalenniveau 
zum Kooperationspartner interpretiert. Eigentlich beinhaltet diese Dimension aber die Abwesenheit hierarchischer Strukturen und privatrechtlicher Beschränkungen in der Kooperationsbeziehung als Charakteristika eines Synergiesystems. Um zu vermeiden, dass auch die Befragten bei der Fragebogenbefragung diese Dimension aufgrund ihrer missverständlichen Bezeichnung missinterpretieren, wurde sie mittels Ergänzung ihrer Bezeichnung um den Hinweis „Unabhängigkeit“ näher bestimmt.

\subsubsection{Adaptierte Dimension „Risikobereitschaft“"}

Um ein Synergiesystem zu etablieren, ist es notwendig, dass die beteiligten Kooperationspartner wechselseitig eine risikoreiche Vorleistung erbringen. Dazu bedarf es von Seiten jedes Kooperationspartners einer gewissen Risikobereitschaft. Es hat sich im Zuge der Expertengespräche gezeigt, dass es dabei jedoch nicht die Bereitschaft ein Risiko einzugehen ist, die ein Synergiesystem entstehen lässt, sondern das Setzen einer tatsächlichen Handlung, die ein Risiko in sich birgt. Erst wenn die Kooperationspartner beide synchron ein Risiko eingegangen sind, indem sie eine risikoreiche Vorleistung erbracht haben, kann sich ein Synergiesystem etablieren. Somit ist diese Dimension um „eingegangenes Risiko“ zu ergänzen und es ergibt sich die endgültige Dimension „Risikobereitschaft und eingegangenes Risiko“.

\subsubsection{Adaptierte Dimension „Cash-Flow-Umsatzrate“}

Um in einem Fragebogen die Frage nach den Cash-Flow-Umsatzraten eines vergangenen Geschäftsjahres und des letzten Geschäftsjahres beantworten zu können, ist für den Befragten ein Aufwand nötig, den er in den meisten Fällen nicht gewillt sein wird auf sich zu nehmen. Außerdem wurden von den Experten während der qualitativen Erhebung Zweifel geäußert ob Befragte überhaupt bereit sind, im Rahmen einer postalischen Fragebogenerhebung konkrete Zahlen anzugeben, die ihren Geschäftserfolg betreffen. Daher wurde von der Abfrage von Zahlenwerten bei der quantitativen Erhebung generell abgesehen und nur die tendenzielle Entwicklung mittels vierstufiger Skala abgefragt. Die Ergebnisse der quantitativen Erhebung werden durch diesen Schritt kaum beeinträchtigt, da die Veränderung des Cash-Flows im Zentrum des Interesses steht und nicht dessen absoluter Wert. Auch die Verbindung zweier Größen in einer Dimension könnte die Befragten überfordern und Nicht- bzw. Falschantworten provozieren, fürchteten die befragten Experten. Um diesem Einwand gerecht zu werden wurde die Dimension in die zwei Dimensionen „Cash-Flow Entwicklung“ und „Umsatz- 
entwicklung“ aufgespaltet. Damit war auch die geplante Dimension „Umsatzwachstum" hinfällig.

\subsubsection{Adaptierte Dimension „Angebotszuschlagsrate“}

Auch hier interessiert die tendenzielle Entwicklung der Zuschläge, die die Unternehmen, deren Geschäftsführer befragt werden, bei Angeboten erhalten und nicht deren absolute Anzahl. Damit ist es auch nicht notwendig, eine Relationszahl abzufragen, die für die Befragten einen Aufwand verursacht (Rechenoperation) den sie vermutlich nicht gewillt wären, auf sich zu nehmen. Die befragten Experten stimmten überein, dass eine Frage nach der tendenziellen Entwicklung der Schwierigkeit, bei Angeboten den Zuschlag zu bekommen, eine wesentlich höhere Chance beizumessen sei, (ehrlich/richtig) beantwortet zu werden. Daher wurde die Dimension in „Angebots-Zuschlags-Entwicklung“ umformuliert.

\subsubsection{Adaptierte Dimension „Anteil der Investitionen am Umsatz“}

Auch diese Dimension war mit den oben ${ }^{281}$ diskutierten Problemen konfrontiert. Daher wurde sie auch aufgespaltet. Die Umsatzkomponente ging in der Dimension „Umsatzentwicklung“ auf, während die Investitionskomponente nun mittels vierstufiger Skala in der Dimension „Entwicklung der Investitionstätigkeit“ erfasst wird.

\subsubsection{Adaptierte Dimension „Kundenzufriedenheit und Stammkundenanteil“}

Es wurde im Zuge der qualitativen Erhebung klargestellt, dass zwar tendenziell nur ein zufriedener Kunde zu einem Stammkunden wird, dass jedoch zahlreiche Fälle denkbar sind, in denen dieser Zusammenhang nicht besteht. Die Zusammenfassung der Zufriedenheit der Kunden eines Unternehmens und des Stammkundenanteils in einer Dimension wäre aber nur dann gerechtfertigt, wenn es sich um eine notwendige Beziehung handelte. Da eine solche Kausalbeziehung zwar als plausibel erscheint, nicht jedoch zwingend ist, wurde diese Dimension in ihre zwei Komponenten „Kundenzufriedenheit“ und „Stammkundenanteil“ aufgespaltet.

\subsubsection{ZUSÄTZLICH IN DIE ERHEBUNG INTEGRIERTE DIMENSIONEN}

Von den im Rahmen der qualitativen Erhebung befragten Experten wurden zahlreiche Dimensionen genannt, die auf Grund ihrer Praxisrelevanz in die Frage-

281 siehe Abschnitt 5.3.2.4. Adaptierte Dimension „Cash-Flow-Umsatzrate“ 
bogenerhebung Eingang finden sollten ${ }^{282}$. Die meisten hielten der Prüfung auf Erhebbarkeit jedoch nicht Stand. Jene Dimensionen, die zusätzlich in die Untersuchung aufgenommen werden konnten, sind im Folgenden dargestellt.

\subsubsection{Zusätzliche Dimension „Mitarbeiterqualifikation“}

Um langfristig erfolgreich zu sein, muss ein Unternehmen sein Know-how ständig weiterentwickeln. Die Träger des Know-how eines Unternehmens sind seine Mitarbeiter (der Geschäftsführer eingeschlossen). Nur hochqualifizierte Mitarbeiter können Innovationen hervorbringen. Innovationen sind für den Erfolg eines Unternehmens langfristig von existenzieller Bedeutung. Dies trifft in besonders hohem Ausmaß auf KMU zu, deren Ressourcen für Outsourcing der Forschungsund Entwicklungstätigkeit tendenziell zu beschränkt sind. Aber auch die Mitarbeiterschulung bindet Ressourcen. Die Entwicklung der Mitarbeiterqualifikation ist für die Beurteilung des Erfolgs eines Unternehmens eine geeignete Dimension, da nur erfolgreiche Unternehmen über genügend freie Ressourcen verfügen, um ihre Mitarbeiter zu schulen und so ihre Qualifikation zu erhöhen. Die Richtung des Kausalzusammenhangs ist für vorliegende Zwecke unerheblich. Es ist hier nur von Bedeutung, dass die Entwicklung der Qualifikation der Mitarbeiter ein brauchbarer Indikator für den Erfolg des Unternehmens darstellt.

\subsubsection{Zusätzliche Dimension „Entwicklung der Beziehungsintensität“}

Die zusätzliche Dimension ergab sich nicht aus den Expertengesprächen, sondern aus einem theoretischen Problem ${ }^{283}$. Diese Arbeit fragt nach der Erfolgswirkung der Selbstverpflichtung in Synergiesystemen. Die interessierende Wirkung (abhängige Variable) ist hierbei der Unternehmenserfolg, der Stimulus ist die Etablierung eines Synergiesystems in der Periode t0 bis t1 und die Selbstverpflichtung stellt die vermutete Ursache (erklärende Variable) dar.

Im vorliegenden Projekt ist t0 als Zeitpunkt zu Beginn der Kooperation somit also als Zeitpunkt vor Etablierung eines Synergiesystems definiert. $t 1$ ist der Zeitpunkt der Befragung. Vor der Durchführung der Befragung ist der Zeitpunkt der möglichen Etablierung eines Synergiesystems nicht bekannt. Es ist daher nicht

282 So wurde beispielsweise vorgeschlagen, es mögen die handlungsdominierenden Werte der Unternehmerpersönlichkeit erhoben werden. Eine solche Erhebung hätte aufgrund ihres Umfanges jedoch nur in einer separaten Untersuchung durchgeführt werden können. (vgl. Fröhlich/Pichler 1988)

283 Dieses theoretische Problem wurde im Zuge einer Diskussion mit o.Univ.Prof.Dkfm.Dr. Josef Mugler aufgeworfen. 
möglich, ex ante den Zeitpunkt t0 entlang der Zeitdimension an einem fixen Zeitpunkt festzusetzen. Der Zeitpunkt der Etablierung ist für jedes Synergiesystem ein anderer. Die Definition eines fixen Zeitpunkts t0 ist daher nicht möglich. Somit ergibt sich durch die Definition von t0 als Zeitpunkt vor der Etablierung des jeweiligen Synergiesystems eine variable Untersuchungsperiode. Eine Auswertung der so erhobenen Daten ist aber dennoch möglich, da im Zuge der Befragung die Bestandsdauer des Synergiesystems abgefragt wird. Mit dieser Information kann nachträglich eine Zeitdimension auf dem Niveau einer Absolutskala konstruiert werden. So wäre sogar eine Untersuchung der Daten auf Kohorteneffekte möglich.

Abb. 18: Auflistung der möglichen Szenarien in der Periode to bis $\mathrm{t} 1$

\begin{tabular}{|c|c|c|c|}
\hline t0 & to bis tl & t1 & Kommentar \\
\hline 0 & 0 & 0 & Unproblematisch: es besteht kein Synergiesystem \\
\hline 0 & 1 & 1 & Unproblematisch: es besteht ein Synergiesystem \\
\hline 0 & 0 & 1 & $\begin{array}{l}\text { Unmöglich. (Wenn zu t } 1 \text { ein Synergiesystem besteht, dann muss es zu- } \\
\text { mindest am Ende der Periode to bis } \mathrm{t} 1 \text { bestanden haben.) }\end{array}$ \\
\hline $\mathbf{0}$ & 1 & $\mathbf{0}$ & PROBLEM! \\
\hline 1 & 0 & 0 & \multirow{4}{*}{$\begin{array}{l}\text { Diese Situationsverläufe sind durch Definition von t0 als Zeitpunkt vor } \\
\text { der Etablierung eines Synergiesystems eliminiert. Daher kann zu t0 } \\
\text { kein Synergiesystem bestehen. }\end{array}$} \\
\hline 1 & 1 & 0 & \\
\hline 1 & 0 & 1 & \\
\hline 1 & 1 & 1 & \\
\hline
\end{tabular}

Quelle: Eigene Darstellung

Dennoch ergibt sich aus der Verwendung einer variablen Untersuchungsperiode ein Problem. Es ist nämlich möglich, dass in der Periode t0 bis t1 ein Synergiesystem existiert hat, das zu t0 noch nicht bestand (siehe Definition von t0) und $\mathrm{zu} \mathrm{t1}$ (Erhebungszeitpunkt) nicht mehr besteht. Trotzdem ist nicht auszuschließen, dass solche zwischenperiodigen Synergiesysteme einen Einfluss auf die abhängige Variable haben. In Abbildung 18 sind alle denkbaren Situationsverläufe in der Periode to bis $t 1$ dargestellt, die sich bezüglich der Existenz eines Synergiesystems aus der Verwendung einer variablen Untersuchungsperiode ergeben. Dabei bezeichnet die Eintragung (1) in der Tabelle den Bestand eines Synergiesystems zum jeweiligen Zeitpunkt, während die Eintragung (0) in der Tabelle eine Situation repräsentiert, in der zu diesem Zeitpunkt kein Synergiesystem besteht.

Dieses Problem ist jedoch mit geringem Mehraufwand im Zuge der Erhebung zu lösen. Mit einer zusätzlichen Dimension „Entwicklung der Beziehungsintensität“ wird abgeklärt, ob sich die Beziehungsintensität im Zeitraum t0 bis t1 verstärkt 
oder abgeschwächt hat. Hat die Intensität der Kooperationsbeziehung in der Periode t0 bis tl abgenommen, so handelt es sich nicht um ein Synergiesystem und der Fall wird aus der Untersuchung ausgeschlossen.

\subsection{Endgültige Dimensionen}

Durch die qualitative Erhebung konnten die meisten der vorläufigen Dimensionen bestätigt werden. Nur eine Dimension musste verworfen werden, einige wurden adaptiert und zwei Dimensionen konnte zusätzlich identifiziert werden. Abbildung 19 bietet eine Zusammenschau der Dimensionen der Variablen, die endgültig für die quantitative Untersuchung verwendet wurden.

Abb. 19: Die endgültigen Dimensionen der Variablen - ein Überblick

\begin{tabular}{|c|c|c|}
\hline $\begin{array}{l}\text { Kategorisierungsvariable } \\
\text { „Synergiesystem“ }\end{array}$ & $\begin{array}{l}\text { Erklärende Variable } \\
\text { „Selbstverpflichtung“ }\end{array}$ & $\begin{array}{c}\text { Abhängige Variable } \\
\text { „Erfolg“ }\end{array}$ \\
\hline $\begin{array}{l}\text { Dimension aufgrund der } \\
\text { Definition der Unter- } \\
\text { suchungseinheiten: } \\
\text { Beziehung zwischen Un- } \\
\text { ternehmen } \\
\text { Dimensionen Kooperation: } \\
\text { Transaktionsdimension: } \\
\text { - Selbständigkeit } \\
\text { - Freiwilligkeit } \\
\text { - Abgestimmtes Verhalten } \\
\text { - Organisierte Beziehung } \\
\text { Zeitdimension: } \\
\text { - Nicht kurzfristige } \\
\text { Perspektive } \\
\text { Dimensionen } \\
\text { Synergiesystem: } \\
\text { Koordinationsdimension: } \\
\text { - Gleichberechtigung } \\
\text { - Möglichkeit der einseiti- } \\
\text { gen Defektion und } \\
\text { Systemgefährdung } \\
\text { Flexibilität und } \\
\text { Unabhängigkeit }\end{array}$ & $\begin{array}{l}\text { Vorgelagerte } \\
\text { Dimensionen: } \\
\text { - } \quad \text { Erfahrung } \\
\text { - } \text { Vertrautheit } \\
\text { - } \text { Individuelle } \\
\text { Reputation } \\
\text { - Kollektive Reputation } \\
\text { - Perzepierte Geschichte } \\
\text { - Risikobereitschaft und } \\
\text { eingegangenes Risiko } \\
\text { Selbstvertrauen } \\
\text { Nachgelagerte } \\
\text { Dimension: } \\
\text { Unaufgeforderte Er- } \\
\text { höhung der eigenen } \\
\text { Gefahr }\end{array}$ & 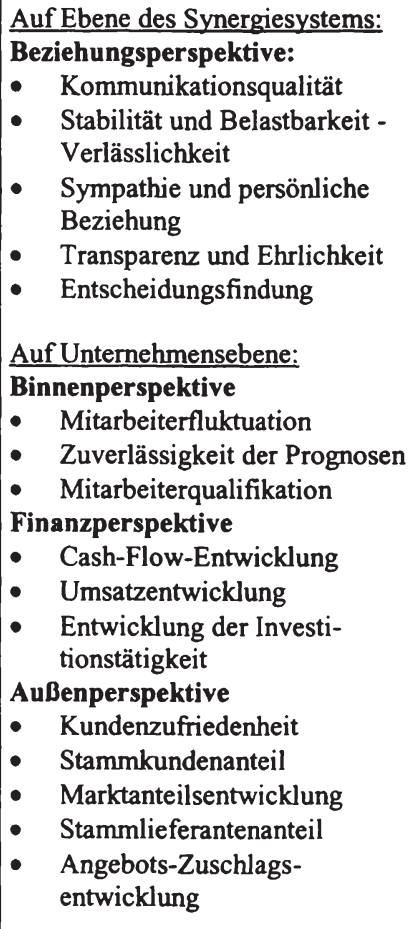 \\
\hline
\end{tabular}


Jetzt, da die endgültigen Dimensionen der Variablen feststehen, ist im Allgemeinen noch zu klären, wie die einzelnen Dimensionen zueinander in Beziehung stehen und im Speziellen, wie die Dimensionen der Variablen Erfolg aufeinander aufbauen und eine Messung des Erfolgs der Unternehmen, die in eine Kooperation eingebunden sind, ermöglichen. Dazu ist für jede latente Variable ein Messmodell zu entwickeln. Die Ausprägungen der latenten Variablen sind nicht direkt erhebbar. Daher wurden für jede latente Variable eine bestimmte Anzahl von Dimensionen erdacht, welche die latente Variable möglichst vollständig erfassen. Für die Erfassung der Dimensionen wurden sodann Messvariablen definiert. Die Erhebung der Ausprägungen jeder Messvariablen konnte dann mit jeweils einer Frage im Rahmen der Fragebogenbefragung operationalisiert werden. Diese drei Messmodelle (jeweils ein Messmodell für die drei latenten Variablen „Synergiesystem“, „Selbstverpflichtung“ und „Erfolg“) werden durch ein Strukturmodell miteinander in Beziehung gesetzt. Eine solche Darstellung ermöglicht die Zuordnung der Dimensionen der Variablen „Synergiesystem“ und „Selbstverpflichtung“ zu den Dimensionen der Variablen „Erfolg“. Die Modellierung erfolgt graphisch, da eine solche Darstellung gegenüber der formal-mathematischen Darstellung für den Leser den Vorteil schnellerer Erfassbarkeit bietet.

\subsection{Modellierung der Interdependenzen zwischen den Dimensionen der latenten Variablen - Entwicklung eines Gesamtmodells}

Bei der Entwicklung eines Gesamtmodells der Erfolgswirksamkeit von Selbst verpflichtung bei Synergiesystemen ist in einem ersten Schritt für jede der drei latenten Variablen (Kategorisierungsvariable „Synergiesystem“, erklärende Variable „Selbstverpflichtung“, abhängige Variable „Erfolg“) jeweils ein Messmodell $\mathrm{zu}$ formulieren, das die einzelnen Messdimensionen der Zieldimension (jeweilige latente Variable) systematisch zuordnet. In einem zweiten Schritt ist ein Strukturmodell zu entwickeln, das die latenten Variablen ihrerseits untereinander in Beziehung setzt.

\subsubsection{ENTWICKLUNG DER MESSMODELLE}

\subsubsection{Messmodell der Variablen Synergiesystem}

Die sechs Dimensionen, die das Phänomen Kooperation erfassen, ergänzt um die drei Dimensionen, die andere Formen der Kooperation von Synergiesystemen 
abgrenzen ${ }^{284}$, ermöglichen die mittelbare Erfassung der Ausprägungen der latenten Variablen Synergiesystem. ${ }^{285}$ Es ergibt sich folgendes Messmodell:

Abb. 20: Messmodell der Variablen „Synergiesystem“

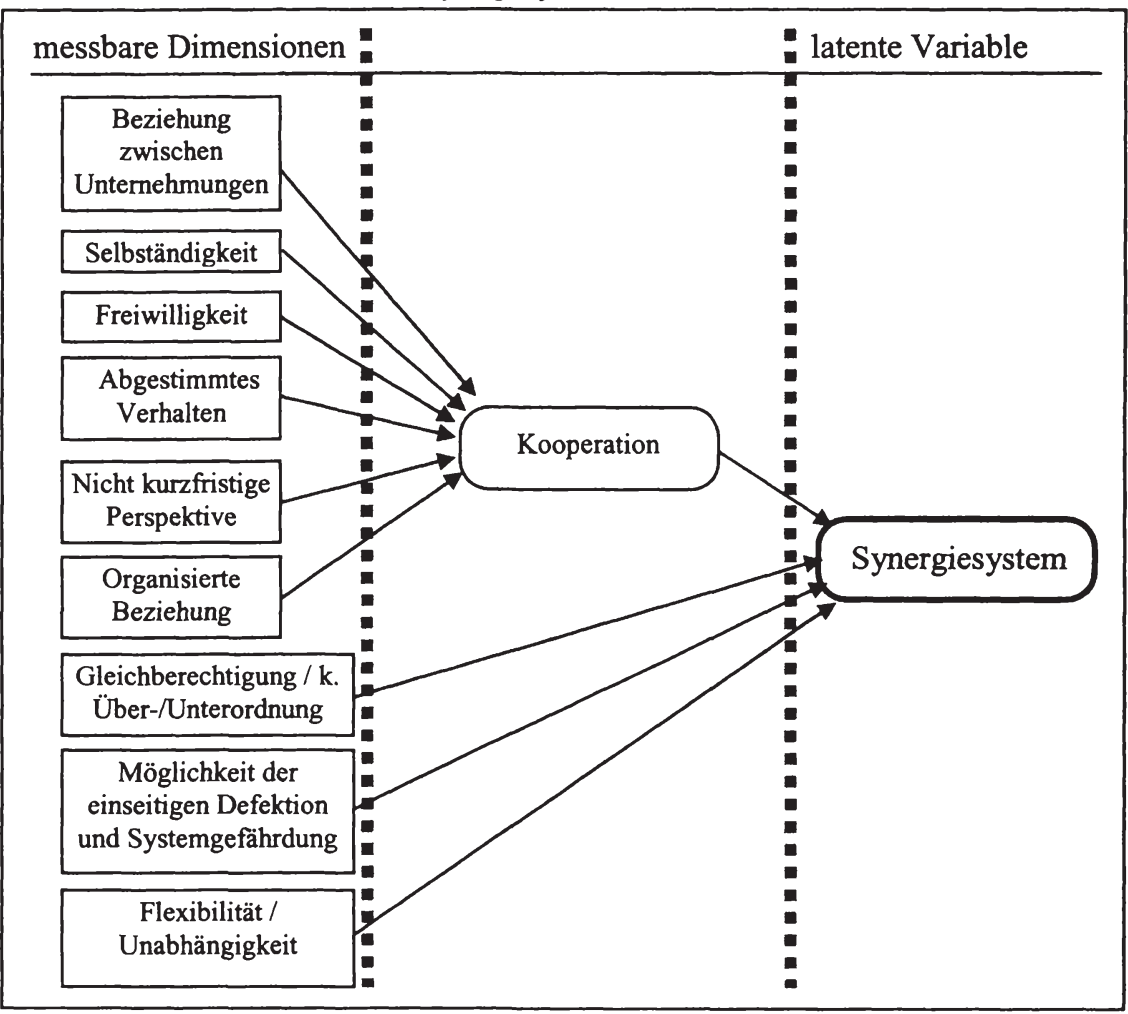

Quelle: Eigene Darstellung

\subsubsection{Messmodell der Variablen „Selbstverpflichtung“}

Das Messmodell der Variablen „Selbstverpflichtung“ besteht aus mehreren vorgelagerten sowie einer nachgelagerten Dimension. Die vorgelagerten Dimensionen sind in zwei Phasen sequenziert. Individuelle Reputation, kollektive Reputation

284 Zur Rechtfertigung der Verwendung genau dieser Dimensionen siehe Abschnitt 5.2.1.2.2. Zusätzliche Charakteristika des Begriffs Synergiesystem

285 Zur Argumentation dieser Form der mittelbaren Erhebung der Ausprägung von Variablen siehe Abschnitt 5.2.3. Abhängige Variable Erfolg 
sowie Erfahrung stellen die Dimensionen der Phase 1 dar und wirken sich auf die Dimension Risikobereitschaft (Phase 2) aus. Die Dimension Erfahrung hat zusätzlich Auswirkungen auf die Dimension Selbstvertrauen (Phase 2) die sich ihrerseits auf die Dimension Risikobereitschaft auswirkt. Ergänzt um die Ausprägungen der Dimensionen Perzepierte Geschichte und Vertrautheit, ergeben die Ausprägungen der Dimensionen Selbstvertrauen und Risikobereitschaft mittelbar die Ausprägung der Variablen Selbstverpflichtung. Die Selbstverpflichtung führt zur Vertrauenshandlung, einer unaufgeforderten Erhöhung der eigenen Gefahr, die als nachgelagerte Dimension im Messmodell erfasst ist. Da zwischen der Selbstverpflichtung und der unaufgeforderten Erhöhung der eigenen Gefahr ein kausaler Zusammenhang besteht, kann im Umkehrschluss ${ }^{286}$ von der unaufgeforderten Erhöhung der eigenen Gefahr auf die Selbstverpflichtung geschlossen werden.

Abb. 21: Messmodell der Variablen „Selbstverpflichtung“

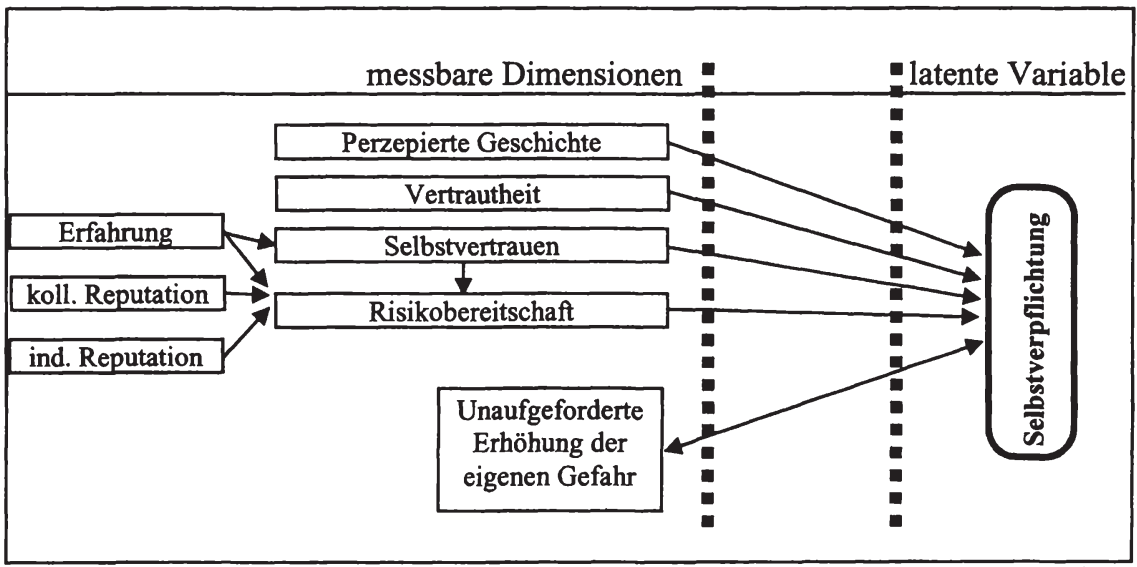

Quelle: Eigene Darstellung

\subsubsection{Messmodell der Variablen „Erfolg“}

Für die Strukturierung dieser rückgekoppelten Effekte bietet sich der formale Raster der Balanced Score Card an, da auch dort Wirkungen von "drivers“ (Stimuli) auf eigentlich interessierende Erfolgsindikatoren abgebildet werden. Der Aufbau eines Kooperationssystems wird hier in diesem Sinne als "driver" analysiert. ${ }^{287}$ Unter Rückgriff auf die Struktur der in Abschnitt 5.2.3. entwickelten

286 Zur Zulässigkeit dieses Umkehrschlusses siehe Abschnitt 3.3. f 287 Fink/Rößl (2004), 62 
Balanced Score Card kann für die Erfassung der latenten Variablen „Erfolg“ ein Messmodell entwickelt werden, bei dem jeweils mehrere Dimensionen einen der vier Teilaspekte der latenten Variablen Erfolg unter Einnahme einer bestimmten Perspektive ergeben. Die Balanced Score Card bietet den formalen Raster für die Strukturierung der rückgekoppelten Effekte, die zwischen den einzelnen Dimensionen dieser Teilaspekte bestehen. Es geht dabei um die Darstellung der Wirkungen eines "drivers“ auf die eigentlich interessierenden Erfolgsdimensionen. Im Falle der vorliegenden Untersuchung stellt die Etablierung eines Kooperationssystems diesen ,driver“ dar. ${ }^{288}$

Abb. 22: Balanced Score Card als formale Struktur der Erfolgsmessung

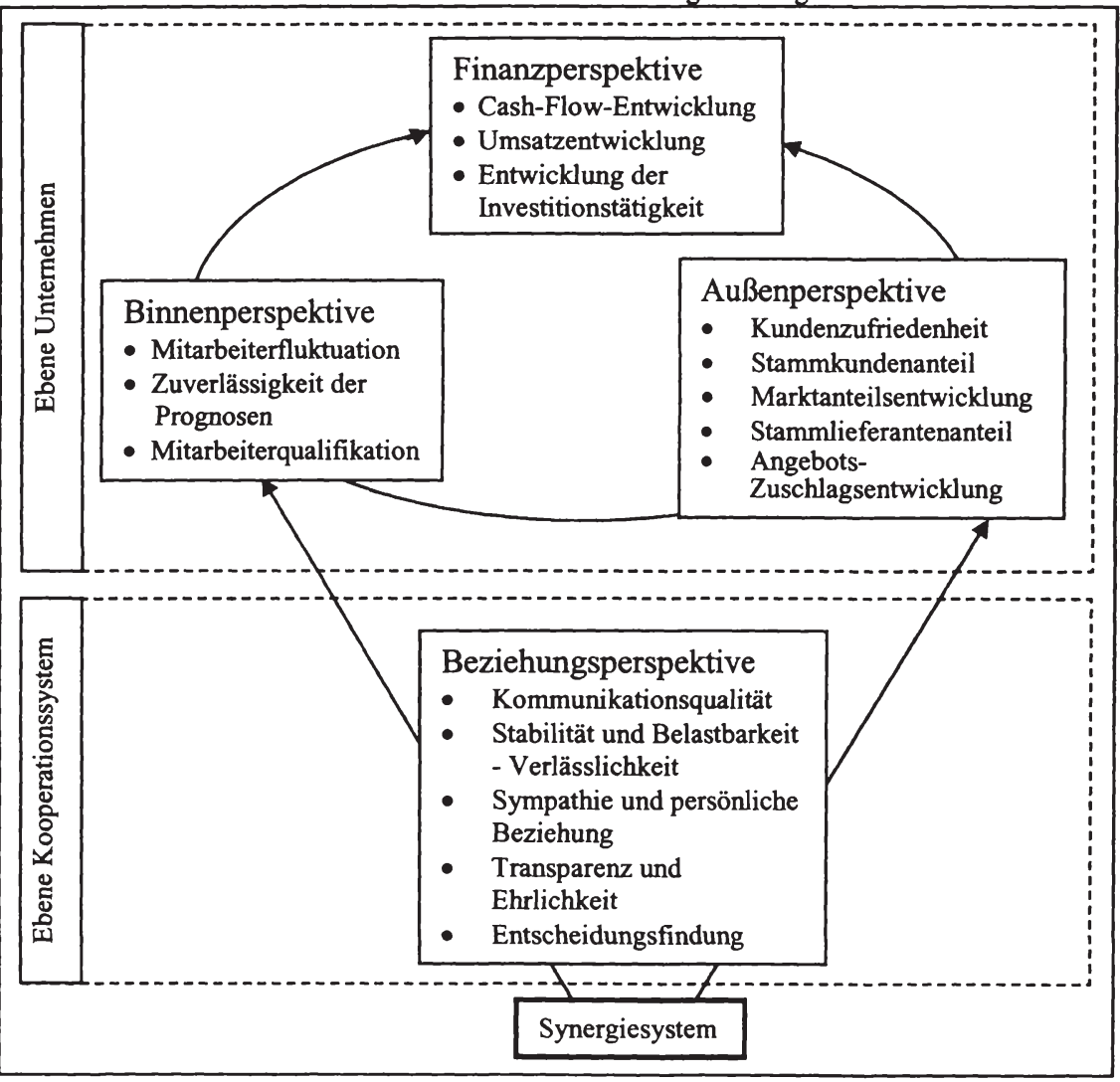

Quelle: Eigene Darstellung 
Abb. 23: Messmodell der Variablen „Erfolg“

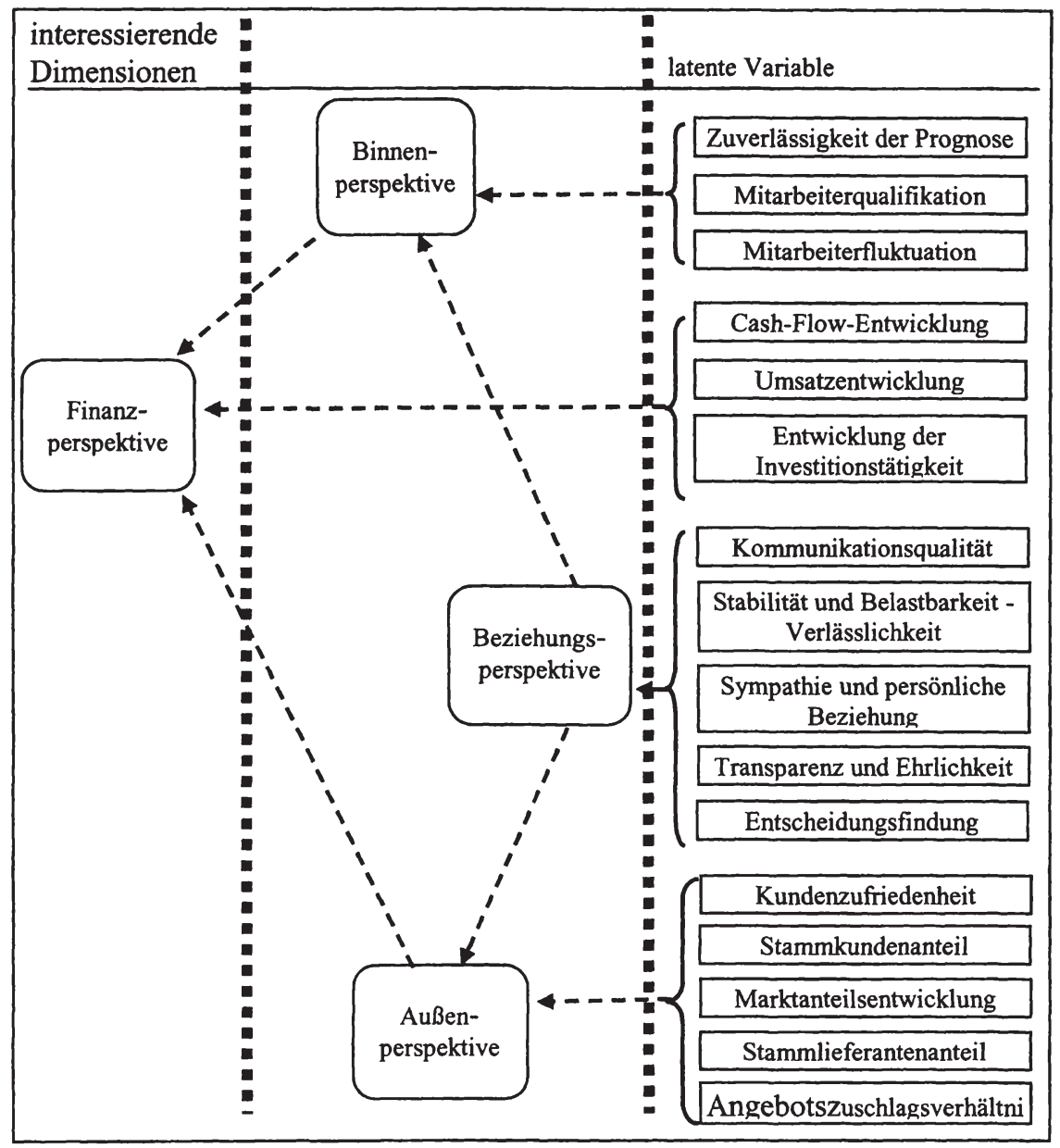

Quelle: Eigene Darstellung

Die Dimensionen Kommunikationsqualität, Stabilität und Belastbarkeit - Verlässlichkeit, Sympathie und persönliche Beziehung, Transparenz und Ehrlichkeit sowie Entscheidungsfindung erfassen den Erfolg auf der Ebene des Kooperationssystems aus der Beziehungsperspektive. Die Dimensionen Mitarbeiterfluktuation, Zuverlässigkeit der Prognosen und Mitarbeiterqualifikation ergeben den unternehmensinternen Erfolg auf der Ebene der einzelnen an einer Kooperation beteiligten Unternehmen. Auf der gleichen Ebene aber aus der Außenperspektive 
betrachten die Dimensionen Kundenzufriedenheit, Stammkundenanteil, Angebots-Zuschlagsentwicklung, Marktanteilsentwicklung sowie Stammlieferantenanteil den Erfolg. Der finanzielle Erfolg der befragten Unternehmen wird durch die Dimensionen Cash-Flow-Entwicklung, Umsatzentwicklung und Entwicklung der Investitionstätigkeit erfasst.

Die Struktur der entwickelten Balanced Score Card unterstellt folgende Dependenzen zwischen den einzelnen Teilaspekten der latenten Variablen Erfolg: Die Entwicklung der in den Dimensionen der Beziehungsperspektive erfassten Aspekte der Kooperationsbeziehung hat Auswirkung sowohl auf die Dimensionen, die unter Binnenperspektive subsummiert sind als auch auf jene Dimensionen, die unter Außenperspektive zusammengefasst sind. Der Erfolg eines Unternehmens nach innen (Binnenperspektive) und nach außen (Außenperspektive) wirkt sich - möglicherweise mit einer gewissen zeitlichen Verzögerung auch auf Dimensionen aus, die mit dem finanziellen Erfolg des Unternehmens in einem Zusammenhang stehen. Diese Dimensionen ergeben die Finanzperspektive. Es handelt sich dabei um die Dimensionen „Cash-Flow-Entwicklung“, „Umsatzentwicklung“ und „Entwicklung der Investitionstätigkeit“. Diese Zusammenhänge ergeben folgendes Modell für die Messung der latenten Variablen „Erfolg“:

\subsubsection{ENTWICKLUNG DES STRUKTURMODELLS}

Nachdem für jede der drei latenten Variablen ein Modell entwickelt wurde, wie die relevanten Ausprägungen mittelbar erhoben werden können, hat noch bestimmt zu werden, wie die einzelnen Messmodelle zueinander in Beziehung stehen.

Es wurde in Abschnitt 3 gezeigt, dass die Selbstverpflichtung der Kooperationspartner eine notwendige Voraussetzung für die Etablierung eines Synergiesystems darstellt. ${ }^{289}$ Somit ist die Selbstverpflichtung der Entstehung eines Synergiesystems logisch vorgelagert. Um diese Kausalbeziehung im Strukturmodell abzubilden, ist in Abbildung 24 ein Pfeil eingetragen, der bei der latenten Variablen „Selbstverpflichtung“ seinen Ausgang hat und bei der latenten Variablen „Synergiesystem" endet.

289 Der Grund dafür ist, dass die bedingungslose Selbstfestlegung der Kooperationspartner auf die Handlungsoption ,kooperatives Verhalten" und die damit getroffene Entscheidung gegen defektives Verhalten, eine mögliche Lösung für Situationen doppelter Kontingenz darstellt. Das Initialmoment der Etablierung eines Synergiesystems (= komplexe Transaktionsbeziehung mit langfristiger Erfolgsperspektive) ist die Durchbrechung der wechselseitigen Verhaltensunsicherheit (doppelte Kontingenz). 
In weiterer Folge soll nun die latente Variable „Erfolg“ in das Strukturmodell eingebunden werden. Die Arbeit fragt nach dem Zusammenhang zwischen „Selbstverpflichtung“ und „Erfolg“ im Fall des Bestandes eines „Synergiesystems“. Oben wurde geklärt, dass die Selbstverpflichtung der Etablierung eines Synergiesystems vorausgeht. Daraus ergibt sich, dass der Erfolg mit der Etablierung des Synergiesystems in Zusammenhang steht. In der graphischen Darstellung des Strukturmodells wird dieser Zusammenhang durch einen Pfeil dargestellt, der seinen Ursprung bei der latenten Variablen „Synergiesystem“ hat und bei der latenten Variablen „Erfolg“ endet. Daraus ergibt sich folgende Kausalkette als Strukturmodell für die vorliegende Untersuchung:

Abb. 24: Das Strukturmodell ${ }^{290}$

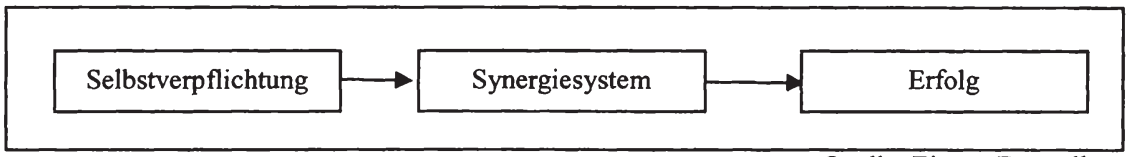

Quelle: Eigene Darstellung

Fügt man nun die Messmodelle in das Strukturmodell ein, so ergibt sich das dieser Arbeit zugrunde liegende Gesamtmodell. Das Gesamtmodell enthält alle für die weitere Untersuchung relevanten Informationen: Erstens sind die latenten Variablen untereinander in Beziehung gesetzt. Zweitens ist geklärt, mit welchen Dimensionen die latenten Variablen erfasst werden und drittens ist angegeben, mit welchen Messvariablen die Ausprägungen der einzelnen Dimensionen erhoben werden. Das Gesamtmodell ist in Abbildung 25 abgebildet.

Nachdem ein Gesamtmodell der Auswirkungen der Selbstverpflichtung auf den Erfolg bei Synergiesystemen erarbeitet wurde, kann nun die empirische Erhebung durchgeführt werden. Dazu wird zuerst ein Fragebogen entwickelt, dessen Struktur dem aufgestellten Modell entspricht und der die Ausprägungen aller im Modell enthaltenen Messvariablen abfragt. Danach sind die relevanten Untersuchungssubjekte zu identifizieren und die erhobenen Daten auszuwerten. 
Abb. 25: Gesamtmodell

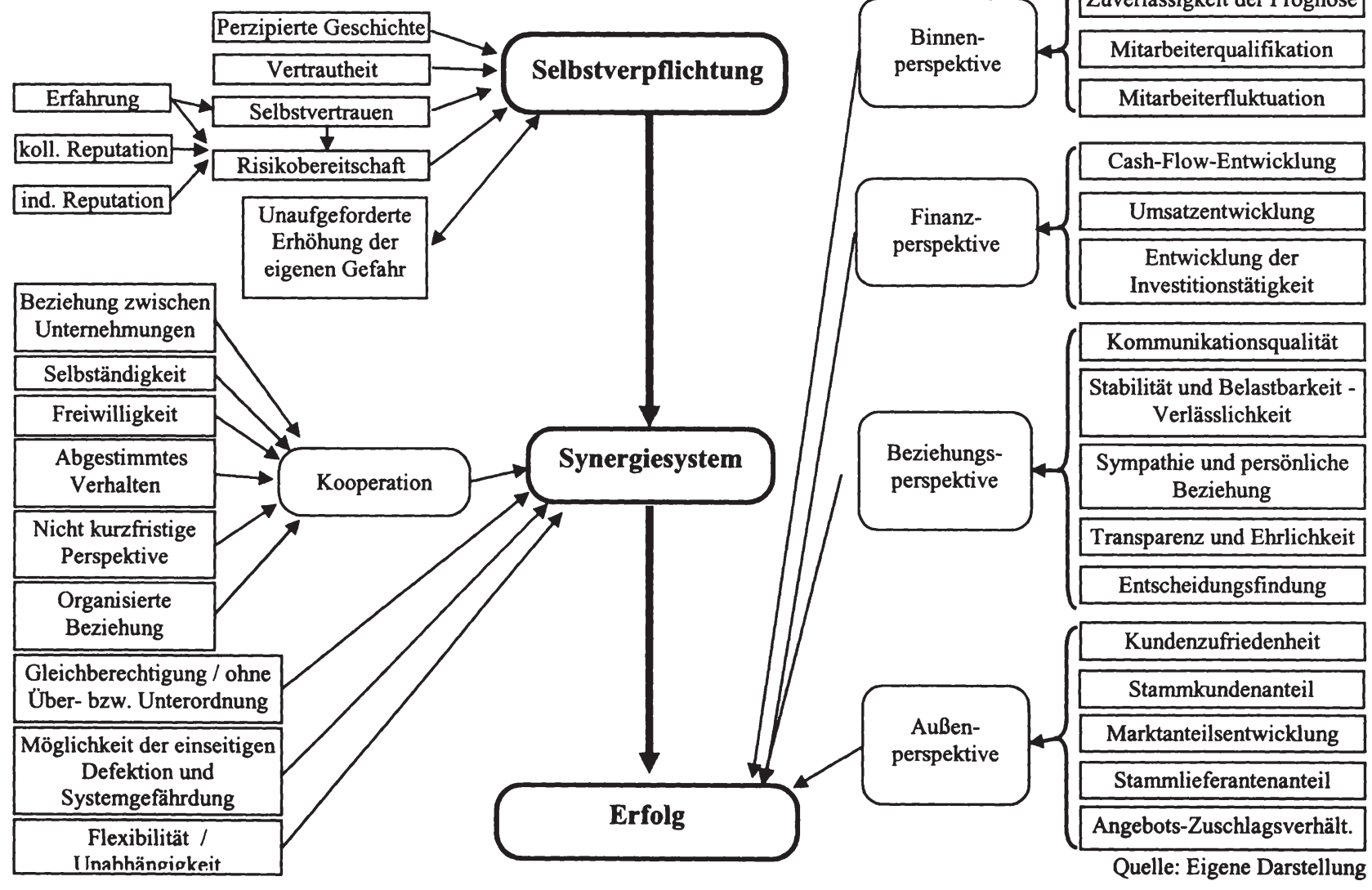




\subsection{Die Quantitative Erhebung}

Die restliche Arbeit beschäftigt sich mit der Konfrontation der in den vorangegangenen Abschnitten dargestellten theoretischen Überlegungen mit der Empirie im Rahmen einer großzahligen Untersuchung. Als Erhebungsmethode bietet sich im vorliegenden Fall eine postalische Fragebogenerhebung an, da diese Methode einerseits eine Streuung der Untersuchungseinheiten über das gesamte österreichische Bundesgebiet ermöglicht, ohne zu kostenintensiv zu sein und andererseits mit vertretbarem Aufwand die Bearbeitung einer großen Stichprobe ermöglicht. $^{291}$

Die Qualität der erhobenen Daten und damit auch der Ergebnisse der Untersuchung hängen bei der postalischen Fragebogenerhebung sehr stark von der Qualität des Erhebungsinstruments, also des Fragebogens, ab. Neben der Formulierung hat auch die Sequenzzierung der Fragen im Fragebogen großen Einfluss auf die Datenqualität. Die Formulierung eines Begleitschreibens, dass die Fragebogenempfänger zur Teilnahme an der Befragung motivieren kann und die optische Aufbereitung der gesamten Postsendung bestimmen mit über die Rücklaufquote und damit über die Aussagekraft der Ergebnisse sowie der, auf deren Basis getroffenen, Aussagen. Bei der Entwicklung und Gestaltung des Erhebungsinstruments ist daher mit größter Sorgfalt vorzugehen und Entscheidungen sind projektbegleitend mit kompetenten Gesprächspartnern zu diskutieren.

\subsubsection{UNTERSUCHUNGSEINHEITEN UND BEFRAGTE}

Der Gegenstand der vorliegenden Untersuchung ist eine besondere Form der Transaktionsbeziehung (Synergiesystem) zwischen Klein- und Mittelbetrieben ${ }^{292}$. Daher stellt das einzelne Unternehmen die Untersuchungseinheit dieser Untersuchung dar. Da das Unternehmen selbst nicht befragt werden kann, stellt die Befragung eines Repräsentanten des Unternehmens den einzig gangbaren Weg dar, um im Zuge einer Befragung Informationen über das Unternehmen zu gewinnen. Entscheidungen über den Aufbau und das Management von Kooperationen liegen in Mittel- und Kleinbetrieben in der Regel in der Verantwortung des Geschäftsführers. Die Adressaten der Fragebögen waren daher die Geschäftsführer der in das Sample aufgenommenen Unternehmen.

291 vgl. Scholl 2003, 47 fowie Dieckmann 2001, 439

292 Die Abgrenzung des Unternehmenstyps „Klein- und Mittelbetrieb“ erfolgt für die empirische Erhebung ausschließlich nach dem Kriterium Anzahl der Beschäftigten, da diese Größe als einzige für statistische Zwecke operational ist. (vgl. Mugler 1999a, 31) 


\subsubsection{ENTWICKLUNG DES ERHEBUNGSINSTRUMENTES}

In den vorigen Abschnitten wurde der argumentative Weg von den latenten Variablen bis zu den einzelnen Messvariablen dargestellt. Dieser Abschnitt beschäftigt sich mit der Entwicklung der konkreten Fragen mit denen im Rahmen der Fragebogenbefragung die Ausprägungen der einzelnen Messvariablen erhoben werden können und mit der Zusammenstellung dieser Einzelfragen zu einem Fragebogen.

Um dem Anspruch auf Klarheit auch bezüglich der inhaltlichen Entwicklung des Textes gerecht zu werden, wird bei der Besprechung der Fragen nach der Struktur des Gesamtmodells ${ }^{293}$ vorgegangen. Demnach werden die Fragen zu den Messvariablen einer Dimension jeweils in einem Unterkapitel besprochen. Die Unterkapitel ihrerseits werden unter der Überschrift zusammengefasst, die Hinweis auf ihre Zugehörigkeit zu einer latenten Variablen gibt.

\subsubsection{GRUNDSÄTZE DER FRAGEBOGENGESTALTUNG}

Um eine so komplexe Aufgabe wie die Gestaltung eines Fragebogens sinnvoll durchführen zu können, ist es hilfreich, sich zuallererst einen Katalog von Grundsätzen zusammenzustellen, die dann das weitere Vorgehen regeln. Die Erstellung eines solchen Grundsatzkatalogs zwingt zur Reflexion aller relevanter Aspekte des bevorstehenden Vorhabens. Probleme werden im Vorfeld bedacht und bearbeitet, sodass sie nicht unberücksichtigt bleiben und später zu Komplikationen führen, die alle bis dahin erledigten Arbeitsschritte in Frage stellen. Bei der Konstruktion des Fragebogens, der für die quantitative Erhebung im Rahmen dieses Projekts angewandt wurde, erfolgte das regelgeleitete Vorgehen nach dem im Folgenden entwickelten Grundsatzkatalog.

\subsubsection{Einfachheit}

Die Forderung nach Einfachheit zwingt zur Verwendung einer einfachen, präzisen sowie verständlichen Sprache. ${ }^{294}$ Wobei sich diese Forderung nicht nur auf die verwendete Grammatik, sondern auch auf die benutzten Vokabeln bezieht. Im Allgemeinen geht es in Bezug auf die Sprache bei dieser Forderung darum, sich bei der Formulierung der Fragen des Fragebogens (Items) in der Sprachwelt der Fragebogenadressaten zu bewegen. Damit sind keine plumpen Anbiederungen ${ }^{295}$

293 Eine Übersichtsdarstellung des Gesamtmodells siehe Abschnitt 5.4.2. Entwicklung des Strukturmodells

294 vgl. Dieckmann 2002, 411

295 vgl. Dieckmann 2002, $410 f$ 
oder ein betont niedriges Sprachniveau gemeint sondern das Bestreben, bei der Formulierung der Fragen eine alltagsähnliche Sprache zu verwenden, die weder jene Befragten mit niedrigem Sprachvermögen überfordert, noch diejenigen mit hohem Sprachvermögen unterfordert. ${ }^{296}$ Das heißt: Der Forscher hat dafür Sorge zu tragen, dass die Befragten im Rahmen einer Fragebogenbefragung nur mit solchen Begriffen konfrontiert werden, die sie kennen und deren Bedeutungsinhalt für alle Befragten möglichst deckungsgleich ist. Somit ist von der Verwendung von Slangausdrücken ${ }^{297}$ oder Fachbegriffen abzusehen. Die Fragen eines Fragebogens haben im Sprachspiel der Fragebogenadressaten stattzufinden und nicht in dem der Sozialwissenschaften. ${ }^{298}$ Es ergibt sich der erste Grundsatz:

G1: Die Sprache soll bekannt, hinreichend einfach, präzise und für die Fragebogenadressaten unmissverständlich sein!

Es darf bei der Konstruktion eines Fragebogens nicht übersehen werden, dass die Befragten nur über Informationen Auskunft geben können, über die sie verfügen oder die sie beschaffen können. ${ }^{299}$ Bezüglich der Antwortschwierigkeit und des Antwortaufwandes gebietet die Forderung nach Einfachheit, dass die Fragen eines Fragebogens nur auf solche Informationen abzielen sollen, die die Fragebogenadressaten unmittelbar präsent haben oder ohne großen Aufwand beschafft werden können. Bei der Beschaffung der Informationen kann der Aufwand entweder das Erfragen, das Nachschlagen in Aufzeichnungen oder der kognitive Aufwand (berechnen, erinnern, einschätzen...) sein. ${ }^{300}$ Jedenfalls ist bei der Fragenformulierung darauf zu achten, dass der gesamte für die Beantwortung der Frage notwendige Aufwand für den Befragten auf ein Minimum reduziert wird. Eine Überforderung oder eine Überbeanspruchung der Geduld der Befragten führt entweder zu Non-Respons oder zu willkürlichem Antwortverhalten und gefährdet den Erfolg der Untersuchung. Als zweiter Grundsatz kann daher formuliert werden:

G2: Gefragt werden soll nur nach Informationen, die der Befragte präsent hat oder sich ohne großen (kognitiven) Aufwand beschaffen kann!

296 vgl. Scholl 2003, $149 \mathrm{f}$

297 vgl. Scholl 2003, 149

298 Der Begriff Sprachspiel wird hier im Wittgenstein'schen Sinn verwendet. Die Konnotationen dieser Begriffswahl sind dem Autor bewusst, fügen sich jedoch ohne große Bruchlinien in das entworfene Gesamtbild.

299 vgl. Scholl 2003, 151

300 vgl. Dieckmann 2001, $413 \mathrm{f}$ 


\subsubsection{Bestimmtheit}

Gerade bei einer Untersuchung wie der vorliegenden, bei der mittels Fragebogenbefragung Zusammenhänge zwischen theoretischen Konstrukten abstrakter Phänomene ergründet werden sollen, ist es wichtig vor der Fragebogenerstellung eine präzise Vorstellung von den zu untersuchenden Phänomenen zu haben. Schon mit der Entwicklung eines Messmodells ist das betreffende Phänomen relativ stark inhaltlich bestimmt. Dem Forscher muss jedoch bewusst sein, dass es sich bei der Operationalisierung ${ }^{301}$ seines theoretischen Modells nicht nur um eine Messbarmachung, sondern gleichzeitig um eine weitere inhaltliche Festlegung (Definition) des Phänomens handelt. Die Formulierung der einzelnen Fragen, die dazu dienen die Ausprägungen der Messvariablen zu erheben, hat im Bewusstsein der inhaltlichen Folgen für die Definition des zu erfassenden Phänomens zu erfolgen. Der Forscher muss bei der Formulierung der Fragen immer wieder überprüfen, ob der Bedeutungsinhalt der einzelnen Fragen seinen inhaltlichen Vorstellungen über das zu untersuchende Phänomen entspricht. Daraus ergibt sich der dritte Grundsatz:

G3: Die Fragenformulierung hat im vollen Bewusstsein ihrer Konsequenzen für
die inhaltliche Bestimmung des zu erfassenden abstrakten Phänomens zu erfolgen.

Eindeutig hat auch die Polung der Items zu sein. Es muss für den Befragten sofort klar sein, welcher Wert der Antwortskala seinem intendierten Antwortverhalten entspricht. In diesem Sinne sind doppelte Verneinungen ebenso ungeeignet wie mehrdimensionale Items. Während die doppelte Verneinung die Gefahr in sich birgt, dass die Befragten irrtümlich eine Antwort geben, die ihren Intentionen widerspricht, sind Antworten auf mehrdimensionale Fragen nicht eindeutig zu interpretieren und daher nicht eindeutig einer Zieldimension zurechenbar. ${ }^{302}$ Es kann dann nicht festgestellt werden, ob die Befragten ihre Antwort auf die eine Dimension der Frage gegeben haben, oder auf die andere. Die Forderung an den Forscher lautet daher:

G4: Die Fragen müssen eindeutig gepolt und eindimensional sein und dürfen keine doppelte Verneinung beinhalten.

Nicht nur die Fragen sondern auch die Antwortkategorien haben genau bestimmt zu sein. Um eine eindeutige Zuordnung der Antwortintentionen der Befragten

301 Zum Begriff der Operationalisierung und zu dessen Kritik siehe Girtler 2001, 50f und die dort zitierte Literatur.

302 vgl. Dieckmann 2001, 412 
einerseits und der gegebenen Antworten zu den Zieldimensionen andererseits zu gewährleisten, müssen die Antwortkategorien nicht nur mit der abgefragten Dimension kompatibel, sondern auch präzise, disjunkt und erschöpfend $\operatorname{sein}^{303}$. Das heißt: Antwortkategorien und abgefragte Dimension müssen auf der selben logischen Ebene liegen (z.B. Häufigkeit, Einstellung, Zustimmung...). Die vorgegebenen Antwortkategorien müssen klar abgegrenzt sein und dürfen sich nicht überlappen, sodass immer nur genau eine Antwortmöglichkeit der Antwortintention der Befragten entspricht. Dies begründet auch die Forderung nach der Bereitstellung aller denkbaren Antwortmöglichkeiten. Eine Ausnahme stellt dabei die Vermeidung einer neutralen Antwortmöglichkeit dar. Sie ist zwar eine denkbare Antwortmöglichkeit und wäre somit als Antwortkategorie anzugeben, um aber zu vermeiden, dass zu viele Befragte von dieser, für sie einfachen Antwortmöglichkeit Gebrauch machen, ist im Lichte der in vorliegender Untersuchung abzufragenden Inhalte, von ihrer Verwendung abzusehen. ${ }^{304}$ Das Fehlen der neutralen Antwortmöglichkeit zwingt die Befragten zu jeder Frage eine Position einzunehmen. Dieses Vorgehen ist damit zu rechtfertigen, dass so der Anteil der auswertbaren Antworten dramatisch steigt, ohne die Verteilung der Antworten auf die restlichen Antwortkategorien wesentlich $\mathrm{zu}$ verändern. ${ }^{305}$ Zusammengefasst lässt sich formulieren:

G5: Die Antwortkategorien müssen präzise, disjunkt (die neutrale Antwortmöglichkeit ausgenommen), erschöpfend und mit der abgefragten Dimension kompatibel sein.

\subsubsection{Unabhängigkeit und Zusammenhang}

Um bei den Befragten keine unerwünschten Konnotationen zu provozieren und die Befragung nicht mit untersuchungsexternen Themen in Zusammenhang $\mathrm{zu}$ bringen, sind stark wertbesetzte Begriffe im Allgemeinen bei der Fragenformulierung zu vermeiden. Solche Begriffe dürfen nur dann eingesetzt werden, wenn sich der Forscher von ihrer Verwendung eine besondere Wirkung auf die Befragten erwartet und eine begründete Erwartung besteht, dass diese Wirkung bei allen Befragten nahezu dieselbe ist. Der unreflektierte Einsatz stark wertbesetzter Begriffe kann zu Verzerrungen des Antwortverhaltens und damit der Ergebnisse der Untersuchung führen, da die von den Konnotationen des Begriffs ausgehenden Wirkungen auf die Fragebogenempfänger höchst unterschiedlich sein können,

303 vgl. Dieckmann 2001, 411

304 vgl. Mayer 2002, 83

305 vgl. Converse/Presser 1986, 36 
nicht vorhersagbar sind und so bei der Interpretation der Ergebnisse der Befragung nicht antizipiert werden können. ${ }^{306}$

G6: Bei der Fragenformulierung sollen stark wertbesetzte Begriffe nur gezielt und reflektiert eingesetzt werden.

Ein weiterer Problemkreis, der im Spannungsfeld von Unabhängigkeit und $\mathrm{Zu}$ sammenhang steht, beinhaltet den Reihenfolgeeffekt und die Aquieszenz. Während der Reihenfolgeeffekt die ergebnisverzerrende Auswirkung des Inhaltes der vorhergehenden Frage auf das Verständnis und damit auf das Antwortverhalten des Befragten bei der darauffolgenden Frage bezeichnet, beschreibt die Aquieszenz die Tendenz der Befragten anstatt auf die Fragen einzeln einzugehen und diese gewissenhaft zu beantworten bei der Beantwortung eines Fragebogens nach einem „Muster“ vorzugehen. Obwohl es sich bei den beiden beschriebenen Problemen um sehr unterschiedliche Phänomene zu handeln scheint, liegt beiden die grundsätzlich gleiche Ursache zugrunde und es können daher auch beide, bei der Fragebogenkonstruktion, mit ein und derselben Maßnahme vermieden werden. Die Ursache beider Phänomene liegt in der Entstehung eines vom Forscher unerwünschten Zusammenhangs. Im Fall des Reihenfolgeeffekts entsteht ein inhaltlicher Zusammenhang zwischen den einzelnen Fragen. Im Fall der Aquieszenz entsteht ein Zusammenhang zwischen der Anordnung der Antwortkategorien der einzelnen Fragen auf dem Fragebogen. Um beide Phänomene zu vermeiden, müssen diese Zusammenhänge dekonstruiert werden. Die Zerschlagung des inhaltlichen Zusammenhangs kann durch Änderungen der Fragensequenzzierung erfolgen. Um die Aquieszenz zu vermeiden, sind negativ gepolte Fragen mit positiv gepolten Fragen zu mischen. ${ }^{307}$ Der Befragte entwickelt beim Ausfüllen des Fragebogens keine Routine, da er sich bei jedem Item die passende Antwortkategorie einzeln überlegen muss. Sollte diese Maßnahme nicht den gewünschten Erfolg erzielen, so kann sie helfen die Personen mit einem hohen Grad an Aquieszenz zu identifizieren. Dennoch ist bei der Konstruktion des Fragebogens darauf zu achten, dass die Befragten während des Ausfüllens in einer einheitlichen Gedankenwelt belassen werden. Daher sollte bei der Fragensequenzzierung dafür Sorge getragen werden, dass unvermittelte Gedankensprünge, die die Befragten verstören oder verwirren könnten in der inhaltlichen Entwicklung des Fragebogens vermieden werden. Aus den Ausführungen ergibt sich der siebente Grundsatz: 
G7: Unterschiedlich gepolte Items sollen so sequenziert werden, dass die Fragen zwar in ihrer inhaltlichen Bedeutung voneinander unabhängig sind, die grundlegende inhaltliche Entwicklung des Fragebogens jedoch nicht gestört wird.

\subsubsection{Bescheidenheit und Notwendigkeit}

Ein grundsätzliches Problem bei Fragebogenbefragungen ist, dass man niemals soviel fragen kann, wie man eigentlich wollte bzw. müsste, um aus den erhobenen Daten gehaltvolle Aussagen treffen zu können. Ein Fragebogen darf nicht zu lange sein, da er sonst Gefahr läuft von den Fragebogenempfängern nicht ausgefüllt zu werden. ${ }^{308}$ Trotzdem soll die Summe der im Fragebogen gestellten Fragen das Forschungsobjekt möglichst vollständig erfassen. Es kommt also zu einem Tradeoff zwischen dem Streben des Forschers nach vollständiger Erfassung des Forschungsobjekts und der Maximierung der Rücklaufquote. Diese Dilemma-situation lässt sich folgendermaßen graphisch darstellen:

Abb. 26: Trade-off zwischen Vollständigkeit und Rücklaufquote

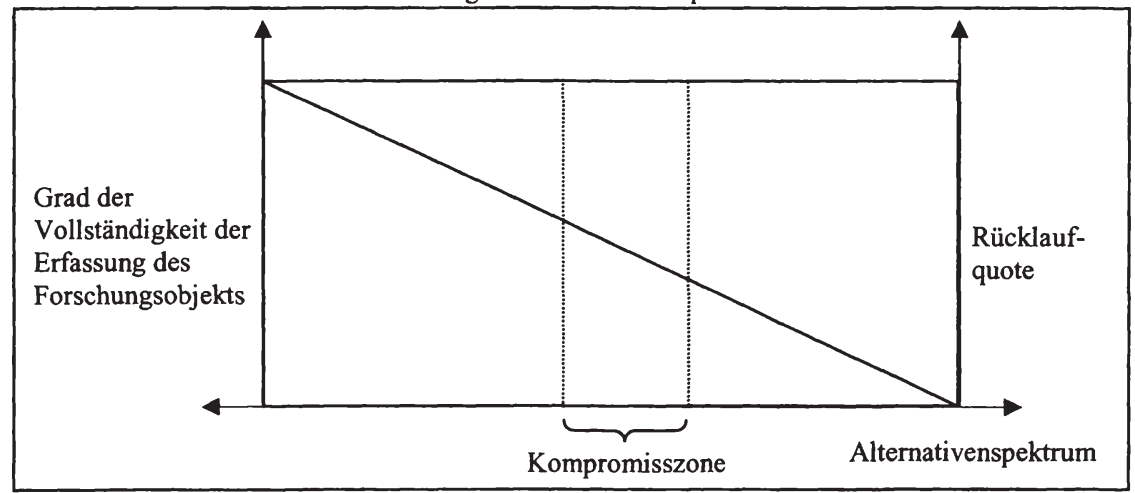

Quelle: Eigene Darstellung

Zwischen diesen beiden konfliktierenden Zielvorgaben ist bei der Fragebogenentwicklung ein Kompromiss zu finden. Die Einnahme einer der beiden Extrempositionen erscheint nicht als sinnvoll, denn eine vollständige Erfassung des Forschungsobjekts bei einer Fragebogenerhebung ohne Rücklauf, ist ebenso wenig wünschenswert wie ein hundertprozentiger Rücklauf bei einer Erhebung mit einem Fragebogen, der das Forschungsobjekt überhaupt nicht erfasst. Geht man davon aus, dass beide Zielvorgaben für den Erfolg einer Fragebogenerhebung

$308 \mathrm{Kirchhoff} / \mathrm{Kuhnt} / \mathrm{Lipp} / \mathrm{Schlawin}$ formulieren: „In der Fragebogenkürze liegt die Antwortwürze." (Kirchhoff/Kuhnt/Lipp/Schlawin 2001, 27) 
gleich wichtig sind, so wird man die jeweiligen Ansprüche symmetrisch so weit reduzieren, bis man sich in der Mitte des Alternativenspektrums trifft. In dieser Kompromisszone liegen die sinnvollen Kombinationen zwischen Vollständigkeitsstreben und Rücklaufquotenmaximierung. Für die Fragebogengestaltung kann der Grundsatz formuliert werden:

G8: In einen Fragebogen sind nur so viele Items aufzunehmen, wie unbedingt notwendig sind, um das Forschungsobjekt hinreichend zu erfassen. Je kürzer der Fragebogen desto besser!

Auch bei der Wahl des Skalenniveaus ist vor übertriebenem Eifer zu warnen. Die Präzision der Messung hat an den Untersuchungsgegenstand angepasst zu sein. Will eine Einstellungsmessung nur grobe Zusammenhänge untersuchen, so ist ein niedrigeres Skalenniveau zu wählen als bei physikalischen Versuchsanordnungen. Allgemein kann gesagt werden, dass bei der Fragebogenerhebung das niedrigste Skalenniveau zu wählen ist, mit dem sich das Untersuchungsobjekt in bezug auf die Forschungsfrage hinreichend erfassen lässt. ${ }^{309}$ Dieser Grundsatz schützt nicht nur den Befragten vor zu detaillierten Fragen, sondern auch den Forschenden vor einer übertriebenen, unnotwendigen Datenflut.

G9: Bei der Fragebogenerhebung ist das niedrigste Skalenniveau zu wählen, das eine für den Untersuchungszweck hinreichende Erfassung des Untersuchungsobjekts gewährleistet.

\subsubsection{Klarheit und Übersichtlichkeit}

Bei der postalischen Fragebogenbefragung sind der Fragebogen und das Motivationsschreiben die einzigen Informationsquellen, die dem Befragten für die Erfassung und die Bearbeitung der an ihn gerichteten Aufgaben zur Verfügung stehen. ${ }^{310}$ Es ist daher alles zu unternehmen, den Fragebogen so klar und übersichtlich wie möglich zu gestalten. Das Ideal wäre der „selbsterklärende“ Fragebogen. Leider ist aber nichts ohne Interpretationsleistung des Rezepienten erfassbar und schon gar kein Fragebogen mit seiner Vielzahl an Items und Antwortkategorien. Dennoch können Maßnahmen getroffen werden, um dem Fragebogenempfänger

309 vgl. Scholl 2003, 163f

310 Von der Möglichkeit des Fragebogenempfängers via Kontaktadresse beim Fragebogenabsender Unklarheiten auszuräumen wird hier abgesehen, da ein solches Vorgehen einen erheblichen Aufwand für den Befragten darstellt, den er in der Regel nicht gewillt sein wird, auf sich zu nehmen. Es ist wahrscheinlich, dass er im Fall von Unklarheiten den Fragebogen nicht fertig ausfüllt und nicht zurückschickt. 
das richtige Erfassen der gestellten Aufgaben zu erleichtern. Die sprachlichen und inhaltlichen Aspekte wurden bereits oben besprochen. Zumeist steht die Anzahl, die Sequenzierung sowie die Formulierung der Items bereits fest, wenn sich der Forscher der graphischen Aufbereitung des Fragebogens zuwendet. Dieser Arbeitsschritt wird häufig vernachlässigt. Er ist jedoch von großer Bedeutung nicht nur für die Rücklaufquote ${ }^{311}$, sondern es sind auch graphische Hilfen wie beispielsweise optische Hervorhebungen (Farben, Balken, Rahmen, Linien und ähnliches), die den Fragebogen optisch strukturieren, Signale geben oder/und den Blick des Befragten leiten und so vor Irrtümern schützen. Grundsätzlich gilt:

G10: Alle graphischen Möglichkeiten müssen ausgeschöpft werden, um den Fragebogen so klar und übersichtlich wie möglich zu gestalten.

\subsubsection{Anonymität}

Der durchschnittliche Befragte gibt ungern Informationen preis. Diese Abneigung ist um so stärker je persönlicher und brisanter die erfragten Informationen sind. Durch Anonymisierung der Fragebögen ist es möglich, dieses Problem zu entschärfen. Daraus resultiert die Forderung:

G11: Den Befragten ist völlige Anonymität zuzusichern und dieses Versprechen darf unter keinen Umständen gebrochen werden.

Nachdem nun elf Grundsätze der Fragebogengestaltung formuliert wurden, kann der Fragebogen für die vorliegende Untersuchung regelgeleitet entwickelt werden. Zuerst werden die Items zu den einzelnen Messvariablen formuliert. Danach folgen Gedanken zu Fragensequenzierung, Fragebogenstrukturierung und zur optischen Aufbereitung des Fragebogens.

\subsubsection{STATEMENTS ALS ITEMS}

Für die vorliegende Befragung wurde die Möglichkeit gewählt, den Befragten im Fragebogen empirische Aussagen ${ }^{312}$ (Statements) zu präsentieren und die Befrag-

311 Ein ansprechend gestalteter Fragebogen kann die Aufmerksamkeit der Adressaten wecken und deren Einstellung der Befragung gegenüber sowie ihre Partizipationsneigung positiv beeinflussen.

312 Empirische Aussagen unterscheiden sich von logischen Aussagen durch ihren empirischen Gehalt. Der empirische Gehalt eines Satzes kann nach K. Popper als der Gehalt eines Satzes definiert werden, durch den der empirische Satz falsifizierbar wird. Ein empirischer Satz kann nach seiner Richtigkeit (oder genauer: nach seiner Falschheit) beurteilt werden. Er ist für Einstellungsmessungen geeignet. Logische Sätze sind per Definition wahr. Ihnen kann ebenso wenig zugestimmt werden, wie sie abgelehnt werden können. 
ten aufzufordern zu den Statements Position zu beziehen. Der Begriff „Frage“ wird in Bezug auf den Fragebogen konsequenterweise im Folgenden durch den Begriff „Item“ ersetzt. Die Statements haben die Form von Feststellungen über Zustände. Die Befragten beziehen Position indem sie den Grad der Übereinstimmung (das Zutreffen) der Statements mit ihrer persönlichen Situationseinschätzung angeben. Diese Form der Einstellungsmessung ist in diesem Fall besonders gut geeignet, da sie den Befragten nicht zwingt, selbst Aussagen zu treffen, sondern nur auf vorgegebene Aussagen zu reagieren. So konnten mögliche Antwortwiderstände reduziert werden.

\subsubsection{ANTWORTKATEGORIEN, MESSSKALA UND SKALENNIVEAU}

Gemäß dem oben formulierten Grundsatz 5 wird für die vorliegende Untersuchung eine gerade Anzahl an Antwortkategorien gewählt. Die Antwortkategorien sollen das gesamte Spektrum der Antwortpräferenzen der Befragten zu den im Fragebogen angeführten Statements erfassen können. Dafür werden den Befragten vier verbalisierte Antwortkategorien zur Verfügung gestellt: „trifft völlig zu“, „trifft zu“, „trifft nicht zu“ und „trifft gar nicht zu“. Ist bei einem Item auf Grund seiner logischen Struktur nur eine dichotome Antwortskala sinnvoll oder vom Informationsgehalt ausreichend, so wird für die positive Antwort die Kategorie "trifft zu“ und für die negative Antwort die Kategorie „trifft nicht zu“ angeboten. Sowohl die Erhebung mittels dichotomer Messskalen als auch mittels der vier verbalisierten Antwortkategorien ergeben Daten auf ordinalem Skalenniveau. Das ermöglicht den Einsatz der Korrelationsanalyse bei der anschließenden Datenanalyse.

\subsubsection{ENTWICKLUNG DER EINZELITEMS}

Dem Leser sei nochmals in Erinnerung gerufen: Jede Einzelfrage (Item) erfasst die Ausprägungen einer Messvariablen. Ein bis zwei Messvariablen repräsentieren jeweils die operationalisierten Dimensionen, von denen mehrere zusammengefasst, jeweils eine der latenten Variablen erfassen. Durch die Formulierung der Einzelitems werden die latenten Variablen endgültig inhaltlich bestimmt. Um für die latenten Variablen Definitionen zu schaffen, die mit den theoretischen Überlegungen in Abschnitt 3 harmonieren, muss bei der Formulierung der Einzelitems stark auf die Begriffswahl geachtet werden. 


\subsubsection{Operationalisierung der Dimensionen der latenten Variablen „Syner- giesystem"}

- Operationalisierung der Dimension „Beziehung zwischen Unternehmen“

Die erste Messvariable für die latente Variable „Synergiesystem“ zielt auf die Dimension „Beziehung zwischen Unternehmen“ ab. Mit ihrer Hilfe sollen aus der Menge der Fragebogenbeantworter jene herausgefiltert werden, die zum Zeitpunkt der Befragung eine Kooperation mit einem anderen Unternehmen unterhalten. Sie werden bei der Datenauswertung der Untersuchungsgruppe zugeteilt. Somit handelt es sich bei diesem Item um einen Filter. Gleichzeitig soll dieses Item als Weiche dienen, denn je nach Antwort auf das Item werden die Befragten auf einen bestimmten Teil des Fragebogens weiterverwiesen. Dadurch müssen sie nur jene Fragen beantworten, die für sie relevant sind und ihr Aufwand bei der Beantwortung des Fragebogens wird minimiert.

Durch die Formulierung dieses Items soll außerdem klargestellt werden, dass in der vorliegenden Erhebung unter Kooperation eine enge Zusammenarbeit zwischen Unternehmen verstanden wird. Es soll eine Abgrenzung gegen Kooperationen zwischen anderen Einheiten, wie beispielsweise Abteilungen, Arbeitsgruppen oder Einzelpersonen geschaffen werden. Das Item wird im Fragebogen daher folgendermaßen formuliert:

Ich unterhalte zur Zeit eine Kooperation (enge Zusammenarbeit) mit einem anderen Unternehmen.

Im Falle einer negativen Antwort auf das obige Item werden die Befragten auf das vierte Blatt des Fragebogens weiterverwiesen, wo jene Items zusammengefasst sind, die für alle Befragten relevant sind. Wird das Item jedoch positiv beantwortet, so erfolgt eine Begriffsbestimmung für den in weiterer Folge häufig im Fragebogen verwendeten Begriff „Kooperationspartner“. Sowohl die Weiterleitung auf die vierte Seite des Fragebogens, als auch die Begriffsbestimmung werden in roter Schrift mit großen Lettern auf den Fragebogen gedruckt, wobei die wichtigsten Satzteile zusätzlich in schwarzem Druck hervorgehoben werden:

Bei der Beantwortung der folgenden Fragen denken Sie bitte an Ihren wichtigsten Kooperationspartner Ihre Ansprechperson in dem Unternehmen, mit dem Sie kooperieren. 
- Operationalisierung der Dimension „Beziehungsintensität“

Als nächste Dimension der latenten Variablen „Synergiesystem“ soll die „Beziehungsintensität" abgefragt werden. Dabei interessiert vor allem die Entwicklung der Intensität der Beziehung zwischen den Kooperationspartnern. Mit diesem Item sollen diejenigen Fälle aus der Untersuchungsgruppe ausgeschlossen werden, bei denen in der Vergangenheit eine Kooperation im Sinne eines Synergiesystems bestand, sich die Intensität der Beziehung im Laufe der Kooperationsbeziehung jedoch so weit vermindert hat, dass zum Zeitpunkt der Messung kein Synergiesystem zwischen den Kooperationspartnern mehr besteht. Als Item, für den Fragebogen wird formuliert:

Die Kooperation ist seit ihrem Beginn intensiver geworden.

- Operationalisierung der Dimensionen „Freiwilligkeit“ und „Selbständigkeit“

Zwei weitere Dimensionen, die Kooperation im hier verstandenen Sinn von anderen Gestaltungsformen von Transaktionsbeziehungen abgrenzen helfen, sind „Selbständigkeit“ und „Freiwilligkeit“. Da diese zwei Dimensionen sehr stark ineinander greifen, werden für die Messvariablen, mittels derer die Ausprägungen dieser zwei Dimensionen erfasst werden sollen, zwei Items formuliert, die auch stark ineinander greifen. Für die Messvariable der Dimension „Selbständigkeit“ ist ein Item zu formulieren, das klarstellt, dass es sich bei dieser Dimension um eine formal-rechtliche und nicht um eine wirtschaftliche Selbständigkeit handelt. Als Item wird daher formuliert:

\section{Ich bin in dieser Kooperation rechtlich selbständig.}

Die Dimension „Freiwilligkeit“" erfasst das Charakteristikum des Synergiesystems, dass jeder Kooperationspartner die Kooperationsbeziehung im Rahmen einer vertraglichen Regelung jederzeit beenden kann. Es ist also abzufragen, ob der befragte Kooperateur durch extrinsische Verhaltensfestlegungen an seinen Kooperationspartner gebunden ist, oder ob er die Kooperation jederzeit beenden kann. Das Item im Fragebogen lautet:

Ich kann die Kooperation jederzeit einseitig beenden. 
- Operationalisierung der Dimension „organisierte Beziehung“

Ein weiteres Unterscheidungsmerkmal eines Synergiesystems ist, dass es sich bei dieser Form der Organisation einer Transaktionsbeziehung um eine organisierte Beziehung handelt, was die Möglichkeit der Selbstthematisierung mit sich bringt. Wenn eine Interaktion Systemeigenschaft besitzt, kann automatisch auch über sie gesprochen werden. Somit lässt sich die Dimension „organisierte Beziehung“ der latenten Variablen „Synergiesystem“ mit folgendem Item als operationalisierte Messvariable erfassen:

Mein Kooperationspartner und ich sprechen über die Kooperation.

- Operationalisierung der Dimension „abgestimmtes Verhalten“

Um zu kooperieren ist es nicht notwendig die gleichen Ziele wie der Kooperationspartner zu haben, sondern die Voraussetzung ist, dass zur individuellen Zielerreichung teilweise die gleichen Mittel eingesetzt werden. Kooperationspartner müssen nicht das gleiche Ziel anstreben, sondern nur einen Teil des Weges gemeinsam bestreiten. Dazu ist es notwendig das Verhalten untereinander abzustimmen. Um diesen Aspekt einer Kooperation bei der quantitativen Erhebung erfassen zu können, wird in den Fragebogen folgendes Item eingefügt:

Ich stimme mein Verhalten mit meinem Kooperationspartner ab.

Die Dimension „abgestimmtes Verhalten“ beinhaltet neben dem oben beschriebenen Aspekt jedoch zusätzlich den Aspekt des aktiven gemeinsamen Vorgehens der Kooperationspartner. Die Kooperationspartner müssen nicht nur für die Zeit der Kooperation ihr allgemeines Verhalten aufeinander abstimmen, sondern für die erfolgreiche Etablierung und den Bestand der Kooperationsbeziehung ist auch ein aktives Setzen von gemeinsamen Handlungen in bestimmten Bereichen, die von der Kooperation betroffen sind, notwendig. Es sind nämlich gerade die erzielten Vorteile aus dem gemeinsamen Vorgehen in gewissen Bereichen der Unternehmensaktivität die Kooperationen für die beteiligten Unternehmen attraktiv machen. Um auch diesen Aspekt der Dimension ,abgestimmtes Verhalten“ bei der Erhebung erfassen zu können, wird folgendes Item in den Fragebogen aufgenommen:

Mein Kooperationspartner und ich gehen in bestimmten Bereichen gemeinsam vor. 
- Operationalisierung der Dimension „,nicht kurzfristige Perspektive“

Kooperationen lassen sich von Markttransaktionen ganz klar anhand ihres längeren Zeithorizonts abgrenzen. Wie in Abschnitt 3 dargelegt, kann die in dieser Arbeit untersuchte Form von Kooperation nur dann entstehen und bestehen, wenn kurzfristige Vorteile zugunsten längerfristiger Vorteile aufgegeben werden. Im Umkehrschluss bedeutet das, dass die Kooperationspartner nicht nach möglichst schnell sichtbaren Vorteilen aus der Kooperation streben dürfen, um eine richtig verstandene Kooperation zu etablieren und zu erhalten. Um die Güte der Erhebung der Dimension ,nicht langfristige Perspektive“ nicht durch den verzerrenden Effekt sozial erwünschter Antworten ${ }^{313}$ zu gefährden, erscheint es sinnvoll, dieses Item gemäß dem Umkehrschluss zu formulieren. Es wird dann nicht danach gefragt, ob der Befragte kurzfristige Vorteile zugunsten längerfristiger Vorteile aufzugeben bereit ist. Vielmehr wird der gegenteilige Fall abgefragt:

\section{Ich möchte durch die Kooperation möglichst schnell sichtbaren Erfolg erzielen.}

- Operationalisierung der Dimension „Gleichberechtigung - ohne Über- bzw. Unterordnung der beteiligten Subsysteme “

Zusätzlich zu den eben entwickelten Items sind für die Operationalisierung der latenten Variablen „Synergiesystem“ Items zu weiteren drei Dimensionen zu formulieren. Diese zusätzlichen Items erfassen die Dimensionen, die das Synergiesystem von anderen Formen der Kooperation unterscheiden. Die erste dieser Dimensionen lautet „Gleichberechtigung - ohne Über- bzw. Unterordnung der beteiligten Subsysteme“. Dabei sind mit Subsystemen die an einer Kooperation beteiligten Unternehmen gemeint. Diese Dimension zerfält in zwei Teilaspekte, denen jeweils eine Messvariable zugeteilt wird. Während die eine Messvariable darauf abzielt den Aspekt der Machtverteilung empirisch fassbar zu machen, will die andere Messvariable den Aspekt des Interessengleichgewichts messbar machen. Wie in Abschnitt 3 argumentiert, müssen für die Etablierung und den Bestand eines Synergiesystems sowohl Macht als auch Kooperationsinteresse annähernd symmetrisch unter den Kooperationspartnern verteilt sein. Für die Messvariable, die darauf gerichtet ist, die Machtverteilung innerhalb der Kooperationsbeziehung zu erheben, wurde folgendes Item formuliert:

Ich denke, mein Kooperationspartner und ich haben gleich starkes Interesse an der Kooperation.

313 vgl. Diekmann 2001, 383f 
Die Messvariable, die dazu dient, das Verhältnis der Stärke der Kooperationsinteressen der beteiligten Transaktionspartner zu messen, wird folgendermaßen operationalisiert:

Bei Entscheidungen, die die Kooperation betreffen, hat die Stimme jedes Kooperationspartners gleich viel Gewicht.

- Operationalisierung der Dimension „Möglichkeit der einseitigen Defektion und Systemdefektion “

Die zweite Dimension, die darauf gerichtet ist, Synergiesysteme von anderen Kooperationen abgrenzbar zu machen, beschreibt die „Möglichkeit der einseitigen Defektion und Systemdefektion“. In engem Zusammenhang mit den oben besprochenen Charakteristika eines Synergiesystems, der symmetrischen Machtund Interessenverteilung, steht auch die beidseitige Möglichkeit sich dem Kooperationspartner gegenüber defektiv zu verhalten. Um eine mehrdimensionale Frage zu vermeiden, muss für jede Richtung möglichen defektiven Verhaltens der Kooperationspartner jeweils ein eigenes Item in den Fragebogen aufgenommen werden:

Ich könnte durch egoistisches Verhalten meinem Kooperationspartner Schaden zufügen.

und:

Mein Kooperationspartner könnte mir durch egoistisches Verhalten Schaden zufügen.

- Operationalisierung der Dimension „Flexibilität - Unabhängigkeit “

Als letzte Dimension der latenten Variablen „Synergiesystem“ ist „Flexibilität Unabhängigkeit" als Merkmal dieser Form der Kooperationsbeziehung zu operationalisieren. Dabei geht es um die grundsätzliche Freiheit der Kooperationspartner aus dem ihnen zur Verfügung stehenden Verhaltensportfolio ohne äußere Einschränkungen zu wählen. Um bei der Messung prüfen zu können, ob dieses Merkmal auf die vom jeweiligen Befragten unterhaltene Kooperationsbeziehung zutrifft, wird folgendes Item in den Fragebogen integriert:

Entscheidungen, die die Kooperation betreffen, werden ausschließlich von meinem Kooperationspartner und mir getroffen. Es gibt keine Einflüsse von außen. 


\subsubsection{Operationalisierung der Dimensionen der latenten Variablen „Selbst- verpflichtung"}

- Operationalisierung der Dimension „Erfahrung“

Gemäß der in Abschnitt 5.2.2.1.2. entwickelten Systematik stellt Erfahrung in Bezug auf Kooperationen Informationsgewinnung dar, bei der die Informationsquelle das eigene Erleben ist und Informationen über einen unbestimmten Kooperationspartner gewonnen werden. Somit sind Erfahrungen im hier verwendeten Sinn eingegrenzt als persönliche Erlebnisse mit dem Phänomen Kooperation. Solche Erlebnisse können die Befragten nur dann gehabt haben, wenn sie schon einmal kooperiert haben. Da Kooperationen zur Zeit in aller Munde sind und die Unternehmen von verschiedenster Seite gedrängt werden, verstärkt Kooperationen einzugehen, erscheint es sinnvoll, dieses Item negativ zu formulieren. Daher werden die Ausprägungen der Messvariablen, die die Dimension „Erfahrung“ erfassen soll mit folgendem Item erhoben:

Diese Kooperation ist meine erste Kooperation.

- Operationalisierung der Dimension „Vertrautheit“

Auch die Arbeitsdefinition der Dimension „Vertrautheit“ ist in der Systematik in Abschnitt 5.2.2.1.2. erklärt. Demnach bedeutet Vertrautheit eigenes Erleben innerhalb einer früheren Kooperation mit dem jetzigen Kooperationspartner. Der Befragte ist genau dann mit seinem jetzigen Kooperationspartner vertraut, wenn er mit diesem Kooperationspartner schon früher einmal im Rahmen einer Kooperation zusammengearbeitet hat. Dieser Sachverhalt lässt sich mit folgendem Item abfragen:

Ich habe mit meinem jetzigen Kooperationspartner schon einmal kooperiert.

- Operationalisierung der Dimension „kollektive Reputation“

Nach der in Abschnitt 5.2.2.1.2. entwickelten Begriffsdefinition stellt kollektive Reputation sozialisiertes Erleben dar, das Informationen über typisches Verhalten einer gewissen Gruppe von Akteuren in Kooperationsbeziehungen erschließt. Bei der vorliegenden Untersuchung ist die relevante Gruppe von Akteuren bestimmt als diejenigen Akteure, die in derselben Branche tätig sind wie der Kooperationspartner des Befragten. Ist ein Erleben sozialisiert, so hat es sich durch soziale Interaktionen in der Gesellschaft oder in Teilen der Gesellschaft verbreitet. Der 
Einzelne kann auf diesen sozialisierten Wissensvorrat durch soziale Interaktion mit anderen Mitgliedern der Gesellschaft zugreifen. Er steht beispielsweise durch Gespräche mit seiner sozialen Umgebung in Kontakt und erhält so auch Informationen über die Branche in der sein Kooperationspartner tätig ist. Um diese Dimension bei der Fragebogenerhebung erfassen zu können, wird folgendes Item in den Fragebogen integriert:

Ich habe vor der Kooperation Gutes über die Branche gehört, in der mein jetziger Kooperationspartner tätig ist.

- Operationalisierung der Dimension ,individuelle Reputation“

Individuelle Reputation wird im vorliegenden Projekt verstanden als sozialisiertes Erleben, das Informationen über einen bestimmten Akteur, für die Gesellschaft oder für Teile der Gesellschaft, erschlossen hat. So wie auch bei der kollektiven Reputation gelangt dieses sozialisierte Wissen über diesen bestimmten Akteur über Sozialkontakte in den Kenntnisbereich des Befragten. Es ist daher folgendes Item zu formulieren:

Ich habe vor der Kooperation Gutes über meinen jetzigen Kooperationspartner gehört.

- Operationalisierung der Dimension ,perzepierte Geschichte“

Der in Abschnitt 5.2.2.1.2. dargestellten Systematik folgend unterscheidet sich perzepierte Geschichte von individueller Reputation dadurch, dass es sich bei letzterer nicht um sozialisiertes Wissen handelt, sondern die Informationsquelle nur dem Fragebogenempfänger bekannt ist. Nichtsozialisiertes Wissen muss aktiv beschafft werden. Die Dimension „perzepierte Geschichte“ erfasst, ob der Befragte Erlebnisberichte anderer Akteure über Interaktionen eingeholt hat, bei denen der Befragte selbst nicht teilgenommen hat. Diese Dimension kann im Fragebogen durch dieses Item erfasst werden:

Ich habe mich vor der Kooperation über meinen Kooperationspartner informiert.

- Operationalisierung der Dimension „Risikobereitschaft - eingegangenes Risiko“

Bei der latenten Variablen "Selbstverpflichtung“ steht die freiwillige Selbstbeschränkung der Akteure im Mittelpunkt. Die Partner legen sich für die Zukunft 
auf ein bestimmtes Verhalten fest, ohne von ihren Transaktionspartnern Sicherheiten zu verlangen. Ein solches Vorgehen birgt für den Akteur das Risiko von den Transaktionspartnern ausgenützt zu werden. Dieses Risiko ist für den Akteur nur auf der Grundlage der Informationen abschätzbar, die er über seine Transaktionspartner zur Verfügung hat. In der quantitativen Erhebung erfolgt die Bemessung der für die Selbstverpflichtung relevante Informationsbasis durch die oben besprochenen Items „Erfahrung“, „Vertrautheit“, „kollektive Reputation“, „individuelle Reputation“ und „perzepierte Geschichte“. ${ }^{314}$ Der Grad der Selbstfestlegung eines Akteurs hängt einerseits von den vorhandenen Informationen über die Transaktionspartner und andererseits vom allgemeinen Umgang des Akteurs mit Risiko ab.

Hat sich ein Akteur im Rahmen einer Kooperation selbstverpflichtet, so ist er sich des Risikos seiner Haltung und der daraus resultierenden Handlungen bewusst. Um die Dimension „Risikobereitschaft - eingegangenes Risiko“ mit dem Fragebogen erfassbar zu machen, muss die relevante Messvariable darauf gereichtet sein, das Risikobewusstsein des Akteurs zu erheben. Zu diesem Zweck wird folgendes Item in den Fragebogen integriert:

\section{Jede Kooperation stellt für die beteiligten Unternehmen ein Risiko dar.}

Ob ein Akteur tatsächlich das, mit der Selbstverpflichtung verbundene, Risiko auf sich nimmt, hängt in großem Ausmaß von seiner allgemeinen Bereitschaft ab, ein Risiko einzugehen. Für risikoaverse Akteure ist Selbstverpflichtung a priori auszuschließen. Um die allgemeine Risikobereitschaft der Befragten zu messen, wird ein weiteres Item in den Fragebogen aufgenommen:

\section{Ich bin bereit ein Risiko einzugehen.}

Will man den Grad der Selbstverpflichtung eines Akteurs messen, so muss bei der Gestaltung des Erhebungsinstruments ein weiterer Aspekt berücksichtigt werden. Das Risiko, dass ein Akteur im Falle seiner Selbstfestlegung innerhalb einer Kooperationsbeziehung eingeht, ist mit der Bedeutung dieser Kooperationsbeziehung für den Erfolg seines Unternehmens in Beziehung zu setzen. Ist ein Akteur in einer Kooperation in hohem Maße selbstverpflichtet, die betroffene Kooperation für sein Unternehmen jedoch in keinster Weise erfolgskritisch, so geht er mit seiner Selbstfestlegung nur ein bescheidenes Risiko ein. Um diesem Umstand bei der Erhebung gerecht zu werden, ist der Einfluss der vom Befragten zum Zeit-

314 Zur Argumentation dieser Zusammenhänge siehe Abschnitt 5.1.2.1.3. 
punkt der Befragung unterhaltenen Kooperation auf den Erfolg seines Unternehmens mit folgendem Item zu erheben:

Die Kooperation hat einen großen Einfluss auf den Erfolg meines Unternehmens.

- Operationalisierung der Dimension „Selbstbewusstsein“

Neben einer ausreichenden Informationsbasis zur Beurteilung der Vertrauenswürdigkeit des Interaktionspartners, einem Risikobewusstsein und einer Risikobereitschaft muss eine weitere Voraussetzung erfüllt sein, damit sich ein Akteur selbstverpflichtet. Der Akteur muss davon überzeugt sein, dass er Rückschläge verkraften könnte, die er erleiden würde, wenn sein Kooperationspartner seine Selbstfestlegung zu eigenen Gunsten ausnützen würde. Ob die Befragten dieses Selbstbewusstsein besitzen, kann mit folgendem Item erhoben werden:

\section{Ich bin überzeugt, Rückschläge verkraften zu können.}

- Operationalisierung der Dimension „unaufgeforderte Erhöhung der eigenen Gefahr“"

Während die oben besprochenen Items die Entstehungsfaktoren der Selbstverpflichtung bei den einzelnen Befragten erheben, will die Dimension „unaufgeforderte Erhöhung der eigenen Gefahr" das Resultat der Selbstverpflichtung des Befragten erfassen. Die Formulierung eines Items, das diese Dimension verlässlich abbilden kann, ist höchst problematisch, da auf ein direkt formuliertes Item kaum unverzerrte Reaktionen zu erwarten sind. Einerseits widerspricht das unaufgeforderte Eingehen einer Gefahr diametral der Unternehmerlogik und andererseits könnte so mancher Befragte ein solches Item benutzen, um seine Selbstsicherheit zu demonstrieren. Um diesen verzerrenden Effekten vorzubeugen, darf sich das Item nicht auf die befragte Person beziehen, sondern muss als generelles Statement formuliert sein. Außerdem erscheint es als sinnvoll, sich des Terminus „Vorschuss“ zu bedienen, um den investiven Charakter der Selbstverpflichtung auszudrücken, denn er impliziert einen späteren Ausgleich. Der Risikoaspekt der Selbstverpflichtung soll dem Befragten genauso vermittelt werden, wie die Tatsache, dass Vertrauen die Basis für Selbstverpflichtung ist. Folgende Formulierung entspricht diesen Ansprüchen:

Damit eine Kooperation gelingt, muss man seinem Kooperationspartner einen Vertrauensvorschuss geben, auch wenn man damit ein Risiko eingeht. 


\subsubsection{Operationalisierung der Dimensionen der latenten Variablen „Erfolg aus der Beziehungsperspektive"}

- Operationalisierung der Dimension „Kommunikationsqualität“

Die Dimension „Kommunikationsqualität“ erfasst die Häufigkeit und die Intensität der kommunikativen Prozesse unter den Kooperationspartnern. Unter Häufigkeit ist nicht die absolute Anzahl der Kommunikationsprozesse pro Zeiteinheit gemeint, sondern der Anteil der realisierten Kontakte an den angestrebten Kontakte mit dem Kooperationspartner. Das heißt, es geht um die Erreichbarkeit des Kooperationspartners. Als Item für den Fragebogen kann somit formuliert werden:

Mein Kooperationspartner ist für mich stets erreichbar.

Die Erreichbarkeit des Kooperationspartners ist mit der Intensität der realisierten Kommunikationsprozesse in Beziehung zu setzen, denn auch häufige oberflächliche oder inhaltsarme Kontakte führen nicht zu einer hohen Kommunikationsqualität. Für die Beurteilung der Qualität der kooperationsinternen Kommunikation ist es notwendig die Befragten im Rahmen des Fragebogens zusätzlich mit folgendem Item zu konfrontieren:

Ich kann meinen Kooperationspartner direkt auf das ansprechen, was mir am Herzen liegt.

- Operationalisierung der Dimension „Stabilität und Belastbarkeit - Verlässlichkeit"

Eine Erfolgsdimension einer nicht nur kurzfristigen Transaktionsbeziehung ist ihre Robustheit gegenüber internen Konflikten. Die Dimension „Stabilität und Belastbarkeit - Verlässlichkeit“ umfasst die Fragen, erstens ob Probleme von den Kooperateuren direkt angesprochen werden können oder eher verdrängt werden und zweitens ob solche Diskussionen, so sie stattfinden, in einem konstruktiven Gesprächsklima ausgetragen werden. Der erste Aspekt kann relativ einfach mit folgendem Item abgefragt werden:

Ich kann mit meinem Kooperationspartner offen über Probleme sprechen.

Den zweiten Aspekt mit einem Fragebogen erfassbar zu machen, ist eine weit schwierigere Aufgabe. Das Problem dabei ist den Begriff „konstruktives Ge- 
sprächsklima“ zu operationalisieren. Für das vorliegende Projekt wurde festgelegt, dass ein konstruktives Gesprächsklima durch seinen Fokus gekennzeichnet ist, in Diskussionen Ergebnisse zu generieren. Als Item für den Fragebogen kann daher formuliert werden:

Diskussionen mit meinem Kooperationspartner führen immer zu einem Ergebnis.

- Operationalisierung der Dimension „Sympathie und persönliche Beziehung“

Dieser Dimension der latenten Variablen „Erfolg aus der Beziehungsperspektive“ liegt die Annahme zugrunde, dass sich Sympathie positiv auf die Qualität der beruflichen Beziehung der Kooperationspartner auswirkt und eine hohe berufliche Beziehungsqualität die Wahrscheinlichkeit persönlicher Kontakte zwischen den Kooperationspartnern erhöht. So kann man von der Tatsache, dass die Kooperationspartner auch privat Kontakt zueinander haben, darauf schließen, dass auch die berufliche Beziehung eine gute ist. Um bei der Erhebung die Qualität der beruflichen Beziehung indirekt zu erfassen, kann daher nach dem Bestand eines privaten Kontakts zwischen den Kooperationspartnern gefragt werden. Als geeignetes Statement wurde folgendes Item formuliert:

\section{Ich habe auch privat Kontakt zu meinem Kooperationspartner.}

Um die Unsicherheiten, die mit einer indirekten Erfassung der Ausprägungen der ersten Messvariablen der Dimension „Sympathie und persönliche Beziehung“ zu reduzieren, wird eine zweite Messvariable definiert. Sie bezieht ihre Rechtfertigung aus dem Zusammenhang, dass interpersonale Beziehungen eher weniger erfolgreich sind, wenn sich die beteiligten Personen als kompliziert empfinden, während sich in erfolgreichen Beziehungen die Partner als unkompliziert beschreiben. ${ }^{315}$ Um die Dimension „Sympathie und persönliche Beziehung“ durch die Befragung mit größerer Sicherheit erfassen zu können, wird auch folgendes Item in den Fragebogen aufgenommen:

Mein Kooperationspartner ist ein komplizierter Mensch.

315 Dieser Zusammenhang lässt sich vermutlich auf den „Fit“ $z$ wischen den beteiligten Personen zurückführen und widerspricht nicht dem Sprichwort, nachdem sich Gegensätze anziehen, denn es sagt weder etwas über die Beziehungsqualität noch über die Bestandsdauer von Beziehungen mit gegensätzlichen Beteiligten aus. 
- Operationalisierung der Dimension „Transparenz und Ehrlichkeit“

Die Dimension „Transparenz und Ehrlichkeit" soll die Aspekte des Informationsaustauschs zwischen den kooperierenden Unternehmen erfassen. Dabei gilt es, mit geeigneten Items im Fragebogen zu erheben, wie gut die Befragten über die Abläufe im Unternehmen ihres Kooperationspartners informiert sind und wie auskunftsbereit das Partnerunternehmen auf Anfragen reagiert. Um nicht mehrdimensionale Items zu generieren, wird für jeden Aspekt dieser Dimension ein eigenes Item formuliert. Der Kenntnisstand der Befragten über die Abläufe im Partnerunternehmen wird mit folgendem Item erhoben:

Ich kenne die Abläufe im Unternehmen meines Kooperationspartners.

Die Auskunftsbereitschaft des Kooperationspartnerunternehmens wird im Zuge der Fragebogenerhebung mit folgendem Item erfasst:

Auf Nachfrage bekomme ich immer die relevanten Informationen über die Abläufe im Unternehmen meines Kooperationspartners.

- Operationalisierung der Dimension „Entscheidungsfindung“

Die Dimension „Entscheidungsfindung“ zerfällt in zwei Aspekte für die jeweils eine Messvariable definiert und folglich jeweils ein Item in den Fragebogen aufgenommen wird. Der eine Aspekt ist der egalitäre Einfluss der Kooperationspartner auf Entscheidungsfindungsprozesse innerhalb der Kooperation. Der zweite Aspekt umfasst die Frage, ob sich die Kooperationspartner innerhalb der Kooperation taktisch verhalten und eigene Interessen vor die Interessen des Kooperationssystems stellen.

Es erscheint jedoch nicht als sinnvoll, den ersten Aspekt direkt abzufragen. Einem solchen Statement, das gleichen Einfluss der Kooperationspartner auf den Entscheidungsfindungsprozess formuliert, würden sowohl solche Befragte ungerechtfertigt zustimmen, die ihren größeren Einfluss verschleiern wollen, als auch solche, die danach trachten, ihren geringeren Einfluss zu verbergen. Das Item wird daher negativ gepolt formuliert:

Bei Entscheidungen habe ich einen größeren Einfluss auf das Ergebnis als mein Kooperationspartner. 
Der zweite Aspekt kann relativ direkt abgefragt werden, gleichwohl das relevante Item betont positiv formuliert wird, um die negative Besetzung des Begriffs „Taktiker“ auszugleichen:

Mein Kooperationspartner ist ein großer Taktiker.

\subsubsection{Operationalisierung der Dimensionen der latenten Variablen „Erfolg aus der Binnenperspektive“}

- Operationalisierung der Dimension „Mitarbeiterfluktuation“

So wie auch bei einigen anderen in Folge angesprochenen Dimensionen, werden die Ausprägungen der Messvariablen der Dimension „Mitarbeiterfluktuation“ für die Elemente der Untersuchungsgruppe zusätzlich mit einem anderen Item erhoben, als das für die Erhebung der Ausprägungen der Variablen bei den Elementen der Kontrollgruppe geschieht. Diese Differenzierung hat ihre Ursache darin, dass bei Befragten, die zum Zeitpunkt der Erhebung eine Kooperation mit einem anderen Unternehmen unterhalten (Untersuchungsgruppe), die Mitarbeiterfluktuation auch auf die Bestandsdauer dieser Kooperation bezogen werden soll und bei den Befragten ohne Kooperation (Kontrollgruppe) dieser Bezug nicht möglich ist. Bei ihnen wird die Mitarbeiterfluktuation nur auf die Zeitspanne der letzten drei Jahre bezogen. Das bedeutet, dass die Mitarbeiterfluktuation bei den Elementen der Untersuchungsgruppe doppelt erhoben wird. Einmal bezogen auf die Bestandsdauer der Kooperation und einmal bezogen auf die Zeitspanne der letzten drei Jahre. So kann der Effekt, den die Kooperation auf diese Größe hat, isoliert werden. Als Item für den Fragebogen ergibt sich für die Mitarbeiterfluktuation, bezogen auf die Bestandsdauer der Kooperation folgendes Item:

Seit Bestand der Kooperation haben weniger Mitarbeiter mein Unternehmen verlassen als zuvor.

Um die Mitarbeiterfluktuation, bezogen auf die Zeitspanne der letzten drei Jahre, zu erheben wird folgendes Item in den Fragebogen aufgenommen:

In den letzten drei Jahren haben mehr Mitarbeiter mein Unternehmen verlassen als zuvor. 
- Operationalisierung der Dimension „Mitarbeiterqualifikation“

Die Begründung für die doppelte Erhebung der Ausprägungen der Messvariablen, welche die Dimension „Mitarbeiterqualifikation“ operationalisiert, ist analog zu den oben angefuihrten Argumenten. Im Unterschied zu den obigen Ausführungen interessiert jedoch hier die Entwicklung der Qualifikation der Mitarbeiter des befragten Unternehmens. Zur Erfassung der Werte dieser Messvariablen werden daher folgende zwei Items in den Fragebogen integriert:

Seit Beginn der Kooperation hat sich die Qualifikation meiner Mitarbeiter erhöht. und

Die Qualifikation meiner Mitarbeiter konnte in den letzten Jahren gesteigert werden.

- Operationalisierung der Dimension „Zuverlässigkeit der Prognosen“

Bei der Operationalisierung dieser Dimension der latenten Variable „Erfolg aus der Binnenperspektive" werden keine Komplikationen gesehen. Die Befragten werden daher im Fragebogen mit folgendem Item konfrontiert:

Die Prognosen meines Unternehmens über den Geschäftsverlauf sind verlässlich.

\subsubsection{Operationalisierung der Dimensionen der latenten Variablen „Erfolg aus der Außenperspektive"}

- Operationalisierung der Dimension „Entwicklung des Angebots-Zuschlagsverhältnisses“

Diese Dimension soll die tendenzielle Entwicklung des Aspekts des Erfolgs aus der Außenperspektive erfassen, wie erfolgreich sich ein Unternehmen bei Auftragsvergaben gegen Konkurrenzangebote durchsetzen kann. Da bei einem positiv formulierten Item eine starke Verzerrung des Antwortverhaltens zu befürchten ist, wird das Item im Fragebogen negativ formuliert. Um Formulierungen wie „weniger Erfolg“ zu vermeiden, wird der geringere Erfolg indirekt durch größere Schwierigkeit einen Erfolg zu erlangen, ausgedrückt. In den Fragebogen wurde somit folgendes Item aufgenommen, um diese Dimension erfassen zu können:

In den letzten drei Jahren ist es schwieriger geworden, bei Angeboten den Zuschlag zu bekommen. 
- Operationalisierung der Dimension „Kundenzufriedenheit“

Die Formulierung eines Items zur Erhebung der Zufriedenheit der Kunden des Befragten ist weniger problematisch als die Aussagekraft einer solchen Einschätzung. Daher wurde bei der Fragebogenkonzeption angedacht, ein weiteres Item in den Fragebogen aufzunehmen, das nach der Durchführung einer Kundenzufriedenheitsmessung im jeweiligen Unternehmen fragt. Solche Messungen sind in der Praxis jedoch auch mit nicht unwesentlichen Problemen der Methodik und Ergebnisinterpretation behaftet, sodass entschieden wurde, dass eine Einbeziehung einer problematischen Vorgehensweise, wie der Kundenzufriedenheitsmessung, nicht geeignet ist, um Probleme der eigenen Erhebung zu kurieren. Gemeinsam mit dem Umstand, dass solche Kundenzufriedenheitsmessungen in KMU eher selten durchgeführt werden, führten diese Überlegungen $\mathrm{zu}$ der Entscheidung, dass die Dimension „Kundenzufriedenheit“ in der Erhebung mit nur einem Item erhoben wird und dabei eine gewisse Ungenauigkeit in Kauf genommen wird. Dieser Item für den Fragebogen lautet:

Meine Kunden sind stets mit den Leistungen meines Unternehmens zufrieden.

- Operationalisierung der Dimensionen „Stammkundenanteil“ und „Stammlieferantenanteil“"

Die Dimensionen „Stammlieferantenanteil“ bzw. „Stammkundenanteil“ zielen auf die Erfassung des Anteiles der Kunden bzw. Lieferanten ab, die regelmäßig mit dem Unternehmen des Befragten Transaktionen durchführen. Im Fragebogen werden diese Dimensionen mit folgenden Items abgefragt:

Die meisten meiner Kunden sind Stammkunden.

und

Die meisten meiner Lieferanten sind Stammlieferanten.

- Operationalisierung der Dimension „Marktanteilsentwicklung“

Diese Dimension erfasst die Entwicklung des Anteiles, den das Unternehmen des Befragten am relevanten Markt hält. In der Fragebogenerhebung wird diese Entwicklung einmal bezogen auf die Bestandsdauer der Kooperation bei den Elementen der Untersuchungsgruppe und ein zweites Mal bezogen auf die Zeitspanne der letzten drei Jahre bei allen Befragten abgefragt. Daraus ergibt sich die Notwendigkeit, für die Erfassung der Dimension „Marktanteilsentwicklung“ folgende zwei Items in den Fragebogen zu integrieren: 
Ich habe seit Bestand der Kooperation meinen Marktanteil vergrößert. und

Ich konnte in den letzten drei Jahren meinen Marktanteil deutlich vergrößern.

\subsubsection{Operationalisierung der Dimensionen der latenten Variablen „Erfolg aus der Finanzperspektive"}

Die oben argumentierte Notwendigkeit zweier Items zur Erfassung der Dimension „Marktanteilsentwicklung“ ist analog auf die drei Dimensionen der latenten Variablen „Erfolg aus der Finanzperspektive“ anzuwenden.

- Operrationalisierung der Dimension „Umsatzwachstum“

Die Erfassung der Dimension „Umsatzwachstum“ erfolgt im Fragebogen mit folgenden zwei Items, wobei das Item, welches das Umsatzwachstum bezogen auf die letzten drei Jahre erfasst, durch das Wort „deutlich“ ergänzt wird. Diese Ergänzung erscheint notwendig, um nicht schon bei geringen Veränderungen die Wahl der Antwortkategorie „trifft völlig zu“ zu provozieren.

Ich konnte seit dem Beginn der Kooperation meinen Umsatz steigern. und

Ich konnte in den letzten drei Jahren meinen Umsatz deutlich steigern.

- Operationalisierung der Dimension „Cash-Flow-Wachstum“

Diese Dimension wird im Fragebogen durch folgende zwei Items repräsentiert:

Ich konnte seit dem Beginn der Kooperation meinen Cash-Flow steigern. und

In konnte in den letzten drei Jahren meinen Cash-Flow deutlich steigern.

- Operationalisierung der Dimension „Entwicklung der Investitionstätigkeit“

Für die Erfassung der Dimension „Entwicklung der Investitionstätigkeit“ werden auch zwei Items in den Fragebogen aufgenommen. Die Formulierung des Items das sich an alle Befragten richtet, ist um den in Klammer gesetzten Hinweis „Geld“ ergänzt. Diese nähere Bestimmung der getätigten Investitionen erscheint notwendig, da sie nicht automatisch aus der inhaltlichen Entwicklung der voran- 
gehenden Fragen des Fragebogens hervorgeht. Es werden daher folgende Items in den Fragebogen integriert:

Seit die Kooperation besteht, habe ich verstärkt in mein Unternehmen investiert. und

Ich habe in den letzten drei Jahren mehr (Geld) in mein Unternehmen investiert als zuvor.

\subsubsection{Zusätzliches Item „Kooperationsdauer“}

Zusätzlich zu den oben besprochenen Items wird noch ein zusätzliches Item in den Fragebogen aufgenommen, das sich nicht einer der latenten Variablen zuordnen lässt. Im Zuge der Planung der quantitativen empirischen Erhebung wurde befunden, dass auch die Bestandsdauer der untersuchten Kooperationsbeziehung bei der Analyse eine wichtige Information zur Datenstrukturierung - z.B. durch Kohortenbildung - sein könnte. Als Item wurde formuliert:

Die Kooperation besteht seit $\square$ Jahren.

\subsubsection{DIE VERBINDUNGSTABELLE}

Abbildung 27 bietet dem Leser eine systematische Zusammenstellung der einzelnen Konkretisierungsstufen der Operationalisierung der Forschungsfrage. 
Abb. 27: Verbindungstabelle

\begin{tabular}{|c|c|c|c|c|}
\hline Variable & $\begin{array}{l}\text { Perspek- } \\
\text { tive }\end{array}$ & Dimension & Formulierung des Items im Fragebogen & $\begin{array}{l}\text { Item } \\
\text { Nr. }\end{array}$ \\
\hline \multirow[t]{13}{*}{$\begin{array}{l}\text { "Synergie- } \\
\text { system“ }\end{array}$} & & $\begin{array}{l}\text { Beziehung zwischen } \\
\text { Unternehmen }\end{array}$ & Ich unterhalte zur Zeit eine Kooperation mit einem anderen Unternehmen. & Filter \\
\hline & & Beziehungsintensität & Die Kooperation ist seit ihrem Beginn intensiver geworden. & 4 \\
\hline & & Selbständigkeit & Ich bin in dieser Kooperation rechtlich selbständig. & 5 \\
\hline & & Freiwilligkeit & Ich kann die Kooperation jeder Zeit einseitig beenden. & 8 \\
\hline & & \multirow{2}{*}{$\begin{array}{l}\text { Abgestimmtes } \\
\text { Verhalten }\end{array}$} & Ich stimme mein Verhalten mit meinem Kooperationspartner ab. & 6 \\
\hline & & & $\begin{array}{l}\text { Meine Kooperationspartner und ich gehen in bestimmten Bereichen gemeinsam } \\
\text { vor. }\end{array}$ & 9 \\
\hline & & Organisierte Beziehung & Mein Kooperationspartner und ich sprechen über die Kooperation. & 36 \\
\hline & & $\begin{array}{l}\text { Nicht kurzfristige Per- } \\
\text { spektive }\end{array}$ & Ich möchte durch die Kooperation möglichst schnell sichtbaren Erfolg erzielen. & 19 \\
\hline & & \multirow[t]{2}{*}{ Gleichberechtigung } & $\begin{array}{l}\text { Ich denke, meine Kooperationspartner und ich haben gleich starkes Interesse an } \\
\text { der Kooperation. }\end{array}$ & 7 \\
\hline & & & $\begin{array}{l}\text { Bei Entscheidungen, die die Kooperation betreffen, hat die Stimme jedes Ko- } \\
\text { operationspartners gleich viel Gewicht. }\end{array}$ & 30 \\
\hline & & \multirow{2}{*}{$\begin{array}{l}\text { Möglichkeit der einsei- } \\
\text { tigen Defektion und } \\
\text { Systemgefährdung }\end{array}$} & $\begin{array}{l}\text { Ich könnte durch egoistisches Verhalten meinem Kooperationspartner Schaden } \\
\text { zufügen. }\end{array}$ & 10 \\
\hline & & & $\begin{array}{l}\text { Mein Kooperationspartner könnte mir durch egoistisches Verhalten Schaden } \\
\text { zufügen. }\end{array}$ & 38 \\
\hline & & $\begin{array}{l}\text { Flexibilität / Unabhän- } \\
\text { gigkeit }\end{array}$ & $\begin{array}{l}\text { Entscheidungen, die die Kooperation betreffen, werden ausschließlich von mir } \\
\text { und meinen Kooperationspartnern getroffen. Es gibt keinen Einfluss von außen. }\end{array}$ & 32 \\
\hline \multirow{2}{*}{$\begin{array}{l}\text { "Selbstver- } \\
\text { pflichtung“" }\end{array}$} & & Erfahrung & Diese Kooperation ist meine erste Kooperation. & 2 \\
\hline & & Vertrautheit & $\begin{array}{l}\text { Ich habe mit meinem jetzigen Kooperationspartner schon früher einmal koope- } \\
\text { riert. }\end{array}$ & 3 \\
\hline
\end{tabular}




\begin{tabular}{|c|c|c|c|c|}
\hline & & Individuelle Reputation & $\begin{array}{l}\text { Ich habe vor der Kooperation Gutes über meinen jetzigen Kooperationspartner } \\
\text { gehört. }\end{array}$ & 11 \\
\hline & & Kollektive Reputation & $\begin{array}{l}\text { Ich habe vor der Kooperation Gutes über die Branche gehört, in der mein } \\
\text { jetziger Kooperationspartner tätig ist. }\end{array}$ & 13 \\
\hline & & Perzepierte Geschichte & $\begin{array}{l}\text { Ich habe mich vor der Kooperation über meinen Kooperationspartner infor- } \\
\text { miert. }\end{array}$ & 14 \\
\hline & & Risikobereitschaft/Ein- & Jede Kooperation stellt für die beteiligten Unternehmen ein Risiko dar. & 15 \\
\hline & & gegangenes Risiko & Ich bin bereit ein Risiko einzugehen. & 20 \\
\hline & & & $\begin{array}{l}\text { Die Kooperation hat einen großen Einfluss auf den Erfolg meines Unter- } \\
\text { nehmens. }\end{array}$ & 12 \\
\hline & & Selbstvertrauen & Ich bin überzeugt, Rückschläge verkraften zu können. & 16 \\
\hline & & $\begin{array}{l}\text { Unaufgeforderte Erhö- } \\
\text { hung der eigenen } \\
\text { Gefahr }\end{array}$ & $\begin{array}{l}\text { Damit eine Kooperation gelingt, muss man seinem Kooperationspartner einen } \\
\text { Vertrauensvorschuss geben, auch wenn man damit ein Risiko eingeht. }\end{array}$ & 18 \\
\hline „Erfolg“ & Beziehungs & Kommunikations- & Mein Kooperationspartner ist für mich stets erreichbar. & 34 \\
\hline & perspektive & qualität & $\begin{array}{l}\text { Ich kann meinen Kooperationspartner direkt auf das ansprechen, was mir am } \\
\text { Herzen liegt. }\end{array}$ & 17 \\
\hline & & Stabilität und Belastbar- & Ich kann mit meinem Kooperationspartner offen über Probleme sprechen. & 37 \\
\hline & & keit - Verlässlichkeit & $\begin{array}{l}\text { Diskussionen mit Kooperationspartner und mir führen immer zu einem Ergeb- } \\
\text { nis. }\end{array}$ & 25 \\
\hline & & Sympathie und persön- & Ich habe auch privat Kontakt zu meinem Kooperationspartner. & 26 \\
\hline & & liche Beziehung & Mein Kooperationspartner ist ein komplizierter Mensch. & 27 \\
\hline & & Transparenz und Ehr- & Ich kenne die Abläufe im Unternehmen meines Kooperationspartners. & 28 \\
\hline & & lichkeit & $\begin{array}{l}\text { Auf Nachfrage bekomme ich immer die relevanten Informationen über die } \\
\text { Abläufe im Unternehmen meines Kooperationspartners. }\end{array}$ & 29 \\
\hline & & Entscheidungsfindung & $\begin{array}{l}\text { Bei Entscheidungen habe ich einen größeren Einfluss auf das Ergebnis als mein } \\
\text { Kooperationspartner. }\end{array}$ & 31 \\
\hline
\end{tabular}




\begin{tabular}{|c|c|c|c|}
\hline & & Mein Kooperationspartner ist ein großer Taktiker. & 33 \\
\hline \multirow[t]{5}{*}{$\begin{array}{l}\text { Binnen- } \\
\text { perspektive }\end{array}$} & \multirow[t]{2}{*}{ Mitarbeiterfluktuation } & $\begin{array}{l}\text { Seit Bestand der Kooperation haben weniger Mitarbeiter mein Unternehmen } \\
\text { verlassen als zuvor. }\end{array}$ & 24 \\
\hline & & $\begin{array}{l}\text { In den letzten drei Jahren haben mehr Mitarbeiter mein Unternehmen verlassen } \\
\text { als zuvor. }\end{array}$ & 49 \\
\hline & \multirow[t]{2}{*}{ Mitarbeiterqualifikation } & $\begin{array}{l}\text { Seit Beginn der Kooperation hat sich die Qualifikation meiner Mitarbeiter er- } \\
\text { höht. }\end{array}$ & 35 \\
\hline & & $\begin{array}{l}\text { Die Qualifikation meiner Mitarbeiter konnte in den letzten drei Jahren } \\
\text { gesteigert werden. }\end{array}$ & 48 \\
\hline & \begin{tabular}{|l} 
Zuverlässigkeit der \\
Prognosen
\end{tabular} & $\begin{array}{l}\text { Die Prognosen meines Unternehmens über den Geschäftsverlauf sind verläss- } \\
\text { lich. }\end{array}$ & 41 \\
\hline \multirow[t]{6}{*}{$\begin{array}{l}\text { Außen- } \\
\text { perspektive }\end{array}$} & $\begin{array}{l}\text { Angebots-Zuschlags- } \\
\text { verhältnis }\end{array}$ & $\begin{array}{l}\text { In den letzten drei Jahren ist es schwieriger geworden bei Angeboten den } \mathrm{Zu} \text { - } \\
\text { schlag zu bekommen. }\end{array}$ & 45 \\
\hline & Kundenzufriedenheit & Meine Kunden sind stets mit den Leistungen meines Unternehmens zufrieden. & 40 \\
\hline & \multirow{2}{*}{$\begin{array}{l}\text { Marktanteils- } \\
\text { entwicklung }\end{array}$} & Ich habe seit Bestehen der Kooperation meinen Marktanteil vergrößert. & 39 \\
\hline & & Ich konnte in den letzten drei Jahren meinen Marktanteil deutlich vergrößern. & 44 \\
\hline & Stammkundenanteil & Die meisten meiner Kunden sind Stammkunden. & 46 \\
\hline & Stammlieferantenanteil & Die meisten meiner Lieferanten sind Stammlieferanten. & 47 \\
\hline \multirow{6}{*}{$\begin{array}{l}\text { Finanz- } \\
\text { perspektive }\end{array}$} & \multirow[t]{2}{*}{ Umsatzwachstum } & Ich konnte in den letzten drei Jahren meinen Umsatz deutlich steigern. & 43 \\
\hline & & Ich konnte seit Beginn der Kooperation meinen Umsatz deutlich steigern. & 22 \\
\hline & \multirow[t]{2}{*}{ Cash-Flow-Wachstum } & Ich konnte in den letzten drei Jahren meinen Cash-Flow deutlich steigern. & 42 \\
\hline & & Ich konnte seit Beginn der Kooperation meinen Cash-Flow deutlich steigern. & 23 \\
\hline & \multirow{2}{*}{$\begin{array}{l}\text { Entwicklung der Inves- } \\
\text { titionstätigkeit }\end{array}$} & Seit die Kooperation besteht, habe ich verstärkt in mein Unternehmen investiert. & 21 \\
\hline & & $\begin{array}{l}\text { Ich habe in den letzten drei Jahren mehr (Geld) in mein Unternehmen investiert } \\
\text { als zuvor. }\end{array}$ & 50 \\
\hline
\end{tabular}




\subsection{8. ÜBERLEGUNGEN ZU FRAGENSEQUENZIERUNG UND FRAGEBOGENSTRUK- TURIERUNG}

Der Fragebogen wird mit der Frage nach dem Bestand einer Kooperation mit einem anderen Unternehmen eröffnet. Dieses Item führt den Befragten in das Thema ein und entscheidet gleichzeitig darüber, welche Teile des Fragebogens der Befragte ausfüllen soll. Während diejenigen Befragten, die eine negative Antwort auf das erste Item geben, nur die vierte Seite des Fragebogens auszufüllen haben, werden diejenigen Befragten, die eine positive Antwort auf die erste Frage geben, auf der Seite eins, zwei und drei des Fragebogens mit Items konfrontiert, die sich auf die unterhaltene Kooperation beziehen. Das Schema ist zur Verdeutlichung in Abbildung 28 dargestellt.

Der Teil der Erhebung, der sich ausschließlich an Kooperateure richtet, wird mit dem zusätzlichen Item „Kooperationsdauer“ eröffnet und beinhaltet alle Items, die Ausprägungen der Dimensionen der latenten Variablen "Selbstverpflichtung“, „Erfolg aus der Beziehungsperspektive“ erheben sowie die speziell für Kooperateure formulierten Items $\mathrm{zu}$ der latenten Variablen „Erfolg aus der Binnenperspektive“, „Erfolg aus der Außenperspektive“ sowie „Erfolg aus der Finanzperspektive". Die drei letztgenannten Perspektiven der latenten Variablen Erfolg werden auch bei den Befragten erhoben, die zum Zeitpunkt der Befragung keine Kooperation mit einem anderen Unternehmen unterhalten. Die dazu in den Fragebogen aufgenommenen Items befinden sich alle auf der vierten Seite des Fragebogens, die sich an alle Befragten richtet.

Die Reihenfolge der Items im Fragebogen folgt in sieben großen Blöcken, der inhaltlichen Abfolge „Synergiesystem“ ” „Selbstverpflichtung“ $\Rightarrow>$ „Erfolg aus der Beziehungsperspektive“ $\Rightarrow>$ „Erfolg aus der Binnenperspektive formuliert für Kooperateure“ $\Rightarrow$ „Erfolg aus der Außenperspektive, formuliert für Kooperateure“ => „Erfolg aus der Finanzperspektive, formuliert für Kooperateure“ $=$ „Erfolg aus der Finanz-, Binnen- und Außenperspektive allgemein formuliert“. Items sind dann nicht nach diesem System gereiht, wenn sie entweder inhaltlich oder von der Formulierung her besser an eine andere Stelle passen und den Fragefluss erleichtern. So werden die Items „Dies ist meine erste Kooperation.“ und "Ich habe mit meinem Kooperationspartner schon einmal kooperiert. " an den Anfang des Fragebogens gereiht, da sie sich einerseits gut als einleitende Fragen eignen und andererseits für den Befragten eine inhaltliche Einheit mit der Frage nach dem Bestand einer Kooperationsbeziehung bilden. 
Abb. 28: Filterwirkung und Struktur des ersten Items

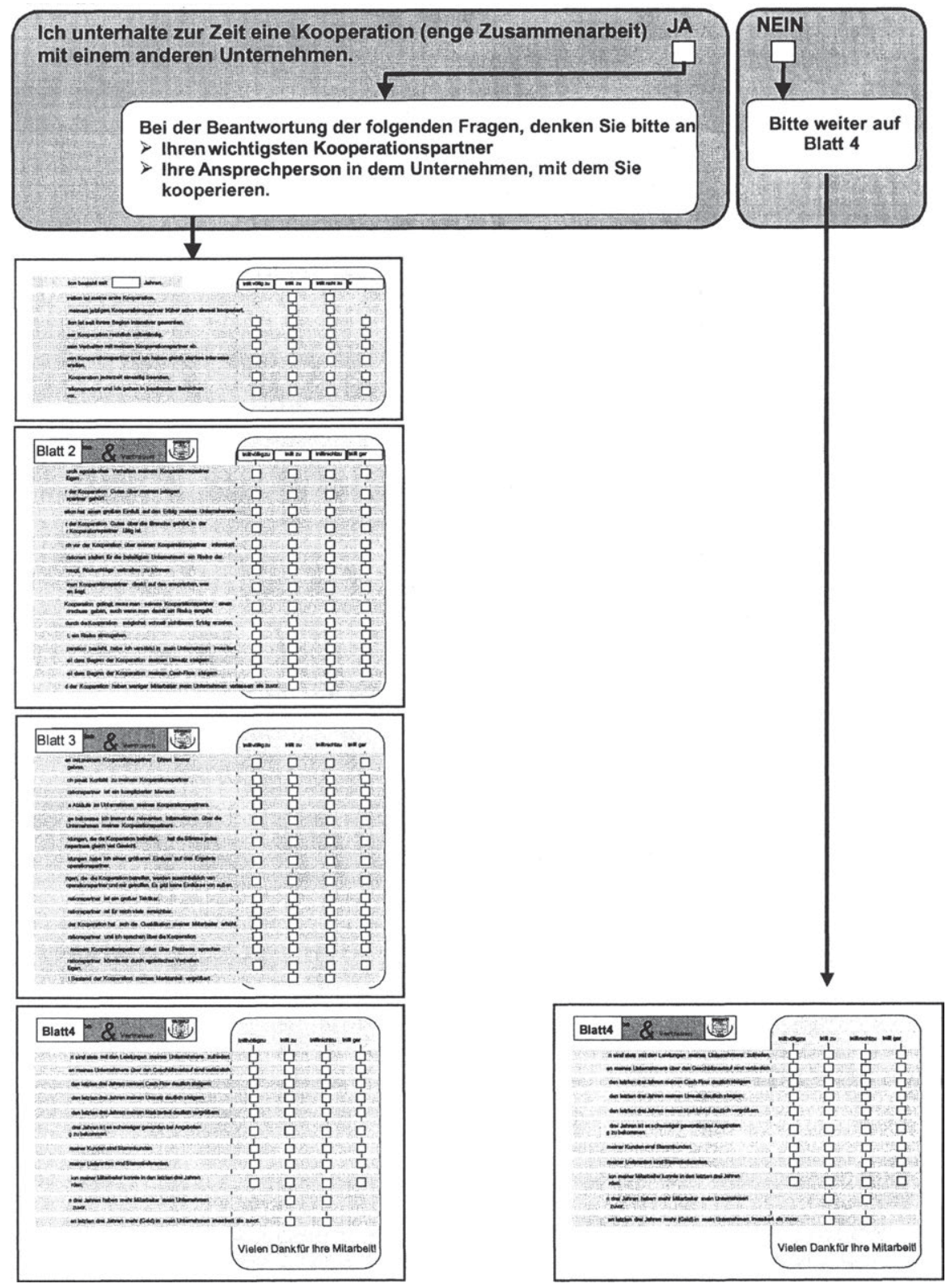

Quelle: Eigene Darstellung 


\subsubsection{UNTERSUCHUNGSZEITRAUM, GRUNDGESAMTHEIT, STICHPROBE UND RÜCK- LAUFQUOTE}

Nun ist die Forschungsfrage in Form eines elaborierten Fragebogens operationalisiert. Die relevanten Größen sind in Variablen übersetzt worden und auf Perspektiven, Dimensionen, Messvariablen und Fragebogenitems heruntergebrochen worden. Die Ausprägungen der Messvariablen werden mit den Items im Fragebogen erfasst. Die Erhebung kann durchgeführt werden.

Die Fragebögen wurden Ende Oktober 2003 an 632 Geschäftsführer kleiner und mittlerer Betriebe ausgeschickt. Eine telephonische Vorankündigung fand aufgrund der großen Stichprobe nicht statt. Die Grundgesamtheit der Untersuchungseinheiten dieser Untersuchung setzt sich aus allen österreichischen KMU zusammen. Die, in das Sample aufgenommenen Elemente der Grundgesamtheit, d.h. die angeschriebenen Unternehmen, stellen eine Zufallsauswahl aus den Datensätzen einer großen Kundendatenbank dar, die sich auf österreichische Klein- und Mittelbetriebe beziehen. Es handelt sich bei diesem Vorgehen selbstverständlich nicht um eine Stichprobenziehung nach dem Zufallsprinzip. Dennoch ist davon auszugehen, dass dieses vereinfachte Auswahlverfahren keinen wesentlichen Verzerrungseffekt auf die Daten hat, da das Unternehmen, das die Kundendatenbank freundlicherweise zur Verfügung stellte, Geschäftskontakte zu allen Betriebsgrößen, Sektoren und Branchen hat und diese Kontakte das gesamte österreichische Bundesgebiet abdecken.

Obwohl bei der Fragebogenaktion die Fragebogenempfänger weder im Voraus telefonisch über das Projekt informiert wurden, noch mittels Nachfassaktion zur Teilnahme angehalten wurden, konnten 138 der 632 Fragebögen ausgewertet werden. Das entspricht einer Rücklaufquote von 21,8 Prozent und stellt eine Datenbasis dar, auf der einerseits gehaltvolle Aussagen getroffen werden können, und die andererseits Rückschlüsse von Merkmalen der Stichprobe auf Merkmale der Grundgesamtheit rechtfertigt.

Im Folgenden ist der Fragebogen dargestellt, der für die Erhebung verwendet wurde. 


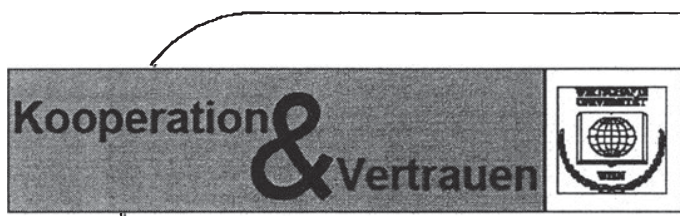

Kontakt: Matthias Fink

Institut für BWL der Klein- und Mittelbetriebe

Wirtschaftsuniversitát Wien

Augasse 2-6, Kem A, 3. Stock

Tel: 0131336 DW 4483

e-mail: Matthias. Fink:aun-wien.ac.at

Sehr geehrte Unternehmerinnen,

die Osterweiterung der Europäischen Union steht unmittelbar bevor. Der verschărfte Wettbewerb stellt gerade für kleine und mittlere Betriebe in Osterreich eine große Herausforderung dar. Um die eigene Wettbewerbsfahigkeit auszubauen und der neuen Situation aktiv entgegen treten zu können, wird es für viele kleine und mittlere Betriebe notwendig sein, Kooperationen einzugehen.

Dieser Fragebogen ist ein wichtiger Teil des Dissertationsprojektes "Kooperation\&Vertrauen" an der

Wirtschaftsuniversităt Wien, das sich zum Ziel gesetzt hat, Kooperationen unter kleinen und mittleren Unternehmen besser zu verstehen. Denn es genugt nicht, die Unternehmer ständig auf die Notwendigkeit von Kooperationen aufmerksam zu machen, sondem es bedarf auch konkreter Hilfestellungen bei der Durchführung der Kooperationen!

Um Antworten auf die Frage "Wie kooperiere ich erfolgreich?" geben zu konnen, mússen wir das Phănomen "Kooperation" besser kennen. Dezu bedarf es Ihrer Mitarbeitl Wenn Sie sich kurz Zeit nehmen und den Fragebogen ausfallen, kann das Projekt zu Ergebnissen kommen und erfolgreich abgeschlossen werden.

Selbstverståndlich handelt es sich um eine anonyme Bofragungl Bitte senden Sie den ausgefullten Fragebogen im beiliegenden Rückkuvert an uns. Wir lassen Ihnen gerne eine Kurzlassung der Ergebnisse zukommen. Um Ihre Anonymitat zu wahren, versenden Sie die beiliegende Antwortkarte bitte getrennt vom Fragebogen.

Vielen Dank fur Ihre Milarbeit,

o.Unw.Prof. Josef Mugier

8o.Univ.Prof. Dietmar Ross

Mag. Matthias Fink 

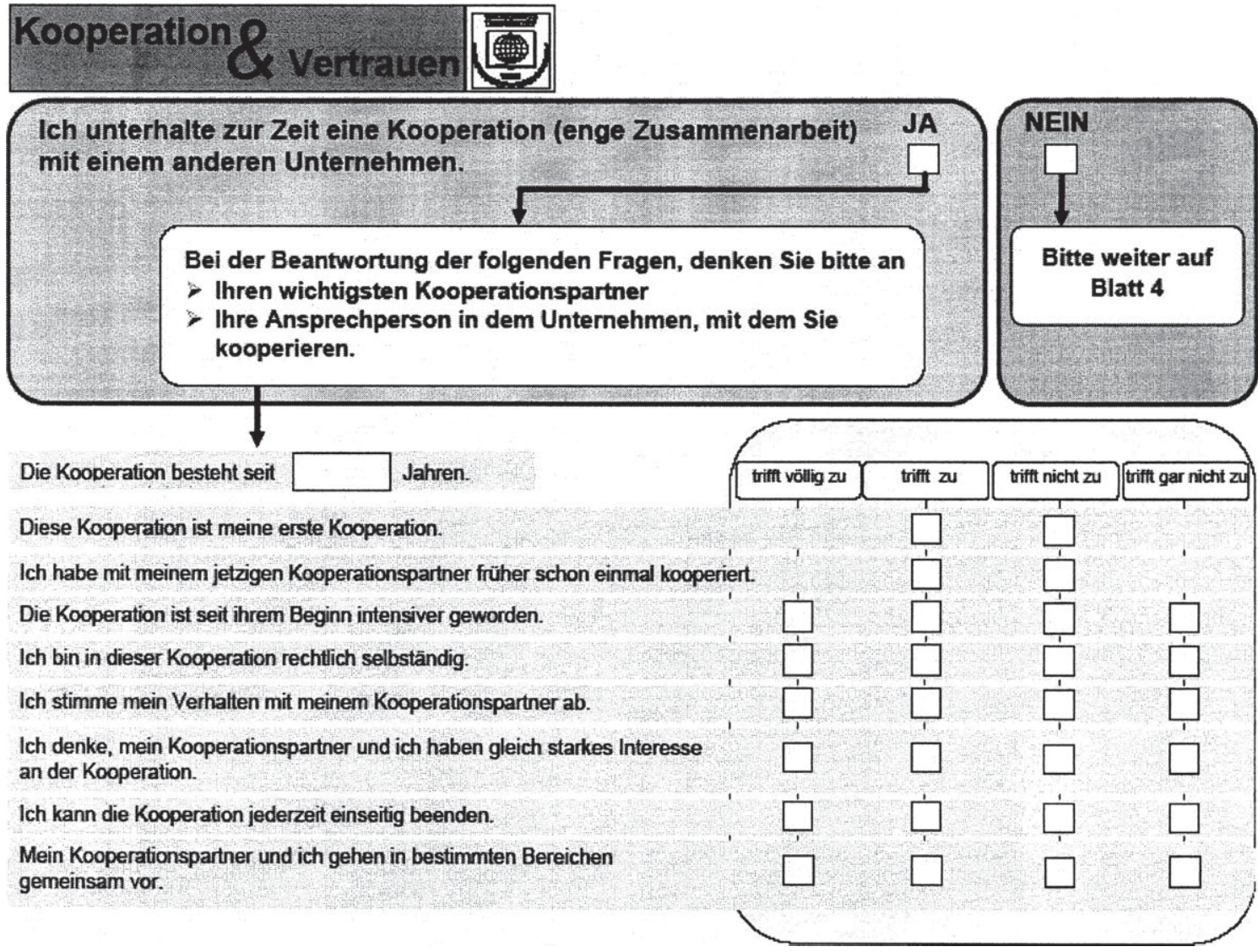


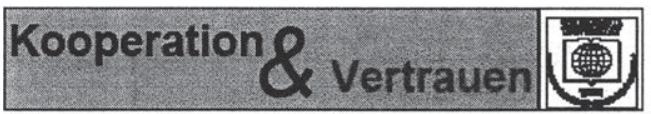

Ich kōnnte durch egoistisches Verhalten meinem Kooperationspartner Schaden zufügen.

Ich habe vor der Kooperation Gutes über meinen jetzigen Kooperationspartner gehört.

Die Kooperation hat einen großen Einfluß auf den Erfolg meines Unternehmens. Ich habe vor der Kooperation Gutes über die Branche gehört, in der mein jetziger Kooperationspartner tätig ist.

Ich habe mich vor der Kooperation über meinen Kooperationspartner informiert. Jede Kooperationen stellen für die beteiligten Unternehmen ein Risiko dar.

Ich bin überzeugt, Rückschläge verkraften zu können.

Ich kann meinen Kooperafionspartner direkt auf das ansprechen, was mir am Herzen liegt.

Damit eine Kooperation gelingt, muss man seinem Kooperationspartner einen Vertrauensvorschuss geben, auch wenn man damit ein Risiko eingeht.

Ich möchte durch die Kooperation möglichst schnell sichtbaren Erfolg erzielen. Ich bin bereit, ein Risiko einzugehen.

Seit die Kooperation besteht, habe ich verstärkt in mein Unternehmen investiert. Ich konnte seit dem Beginn der Kooperation meinen Umsatz steigem.

Ich konnte seit dem Beginn der Kooperation meinen Cash-Flow steigern.

Seit Bestand der Kooperation haben weniger Mitarbeiter mein Untemehmen verlassen als zuvor. 


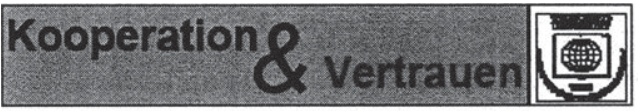

Diskussionen mit meinem Kooperationspartner führen immer zu einem Ergebnis.

Ich habe auch privat Kontakt zu meinem Kooperationspartner.

Mein Kooperationspartner ist ein komplizierter Mensch.

Ich kenne die Abläufe im Unternehmen meines Kooperationspartners.

Auf Nachfrage bekomme ich immer die relevanten Informationen über die Abläufe im Unternehmen meines Kooperationspartners.

Bei Entscheidungen, die die Kooperation betreffen, hat die Stimme jedes Kooperationspartners gleich viel Gewicht.

Bei Entscheidungen habe ich einen größeren Einfluss auf das Ergebnis als mein Kooperationspartner.

Entscheidungen, die die Kooperation betreffen, werden ausschließlich von meinem Kooperationspartner und mir getroffen. Es gibt keine Einflüsse von außen.

Mein Kooperationspartner ist ein großer Taktiker.

Mein Kooperationspartner ist für mich stets erreichbar.

Seit Beginn der Kooperation hat sich die Qualifikation meiner Mitarbeiter erhöht

Mein Kooperationspartner und ich sprechen über die Kooperation.

Ich kann mit meinem Kooperationspartner offen über Probleme sprechen.

Mein Kooperationspartner könnte mir durch egoistisches Verhalten Schaden zưügen.

Ich habe seit Bestand der Kooperation meinen Marktanteil vergrößert.

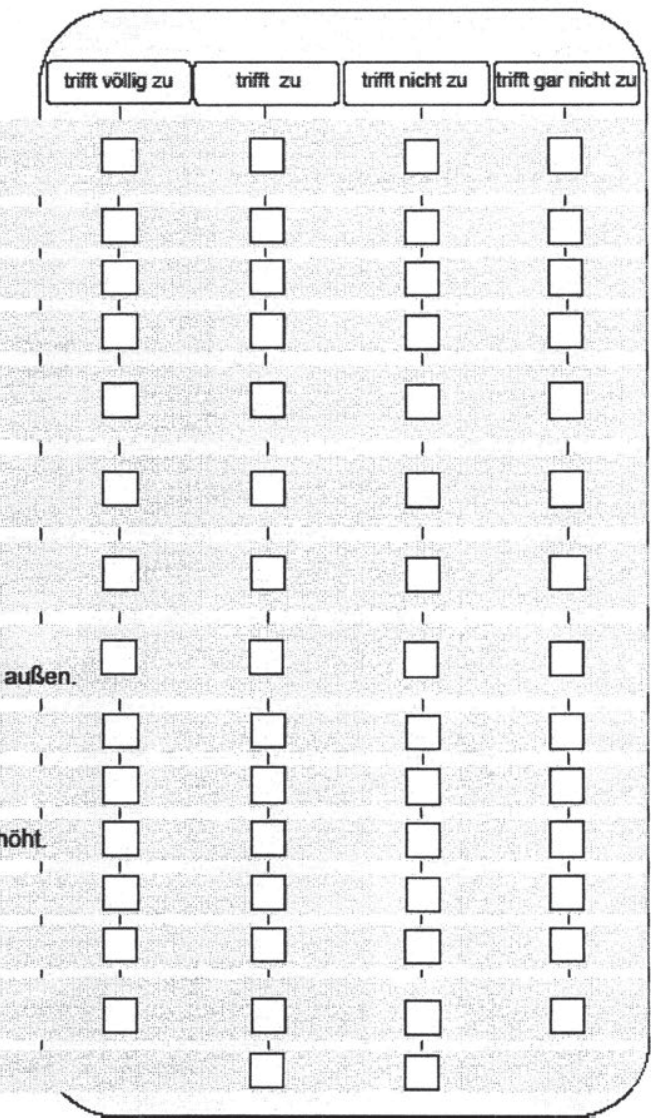




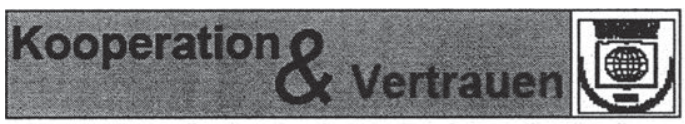

Meine Kunden sind stets mit den Leistungen meines Unternehmens zufrieden.

Die Prognosen meines Unternehmens über den Geschäftsverlauf sind verlässlich.

Ich konnte in den letzten drei Jahren meinen Cash-Flow deutlich steigern.

Ich konnte in den letzten drei Jahren meinen Umsatz deutlich steigem.

Ich konnte in den letzten drei Jahren meinen Marktanteil deutlich vergrößem.

In den lezten drei Jahren ist es schwieriger geworden bei Angeboten den Zuschlag zu bekommen.

Die meisten meiner Kunden sind Stammkunden.

Die meisten meiner Lieferanten sind Stammlieferanten.

Die Qualifikation meiner Mitarbeiter konnte in den letzten drei Jahren gesteigert werden.

In den letzten drei Jahren haben mehr Mitarbeiter mein Unternehmen verlassen als zuvor.

Ich habe in den letzten drei Jahren mehr (Geld) in mein Unternehmen investiert als zuvor.

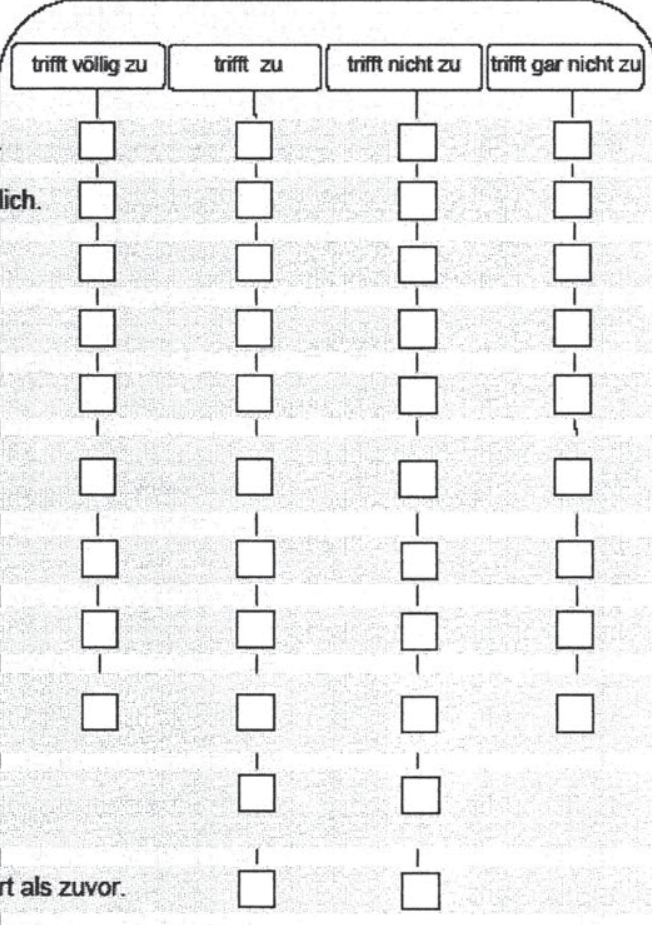

\section{Vielen Dank für Ihre Mitarbeit!}




\subsection{Datenauswertung}

\subsubsection{A POSTERIORI PRÜFUNG DER GÜTE DES ERHEBUNGSINSTRUMENTS}

Bevor auf der Grundlage der erhobenen Daten Aussagen getroffen werden, muss die Qualität der Datenbasis untersucht werden. Nur eine eingehende Prüfung der Güte des vorhandenen Datenmaterials gibt Auskunft über die Aussagekraft der auf dessen Basis getroffenen Aussagen. Daher werden zuerst die Items des Fragebogens darauf untersucht, ob sie geeignet waren, die jeweilige Zieldimension zu erfassen. Diese Prüfung erfolgt mittels Berechnung von Trennschärfekoeffizienten. Danach werden die einzelnen Itembatterien des Fragebogens mit der SplitHalf Methode auf Reliabilität gestestet. Die folgenden Ausführungen werden durch graphische Darstellungen ergänzt.

\section{Exkurs III: Ursachen, Wirkungen und der Pfeil}

Sobald Pfeile als graphische Darstellungen von Kausalbeziehungen herangezogen werden, wird das Dilemma der Identifizierung von Ursache und Wirkung innerhalb einer solchen Kausalbeziehung deutlich. Denn außer wenn die Zeitdimension eine Sequenzierung der Elemente der Kausalkette logisch vorschreibt, ist jede Festlegung der Wirkungsrichtung willkürlich. Das heißt, dass bei Aussagen, die keine Zeitdimension enthalten, jede Ursache als Wirkung und jede Wirkung als Ursache interpretiert werden kann. Die Richtung in der ein Pfeilkopf in einer Grafik zeigt, ist somit jederzeit argumentierbar, genauso wie die Gegenrichtung begründbar wäre.

Warum werden in der vorliegenden Arbeit dennoch Pfeile als Symbol für Kausalbeziehungen herangezogen und nicht einfach Linien, die die Richtung des Wirkungszusammenhangs zwischen kausal aufeinander bezogenen Elementen offen lassen? Der Grund liegt darin, dass mit der Darstellung der Kausalbeziehung als Pfeil die jeweils diskutierte Richtung der Kausalbeziehung betont werden kann. So sind in dieser Arbeit die in den Grafiken enthaltenen Pfeile gemäß der Richtung des Schlusses ausgerichtet, der mit der Kausalbeziehung gerechtfertigt werden soll. Somit stellen die Pfeile in dieser Arbeit keine Repräsentanten von unidirektionalen, Kausalbeziehungen dar, sondern verdeutlichen die gerade betrachtete Wirkungsrichtung der betroffenen Kausalbeziehung. So unterstützen sie graphisch die Argumentation. 


\subsubsection{Prüfung der Güte der Fragebogenitems mittels Trennschärfekoeffizient}

Die Frage, ob die einzelnen Items des Fragebogens geeignet waren, die jeweilige Zieldimension zu erfassen, lässt sich anhand der erhobenen Daten, mittels Berechnung von Trennschärfekoeffizienten beantworten. Dabei werden die erhobenen Werte der einzelnen Items mit den Summenscores der gesamten Itembatterie korreliert. So ergibt sich für jedes Item ein Korrelationskoeffizient - der Trennschärfekoeffizient. Ist der Trennschärfekoeffizient für die Mehrzahl der Items hoch und nur bei einigen wenigen Items niedrig, so ist das ein Anzeichen dafür, dass letztere für die Messung der Zieldimension weniger geeignet waren. ${ }^{316}$ Vereinfacht ausgedrückt wird bei diesem Test untersucht, ob das Antwortverhalten der Befragten über die Einzelitems einer Itembatterie hinweg konsistent war. Folgen die Antworten der Befragten auf ein einzelnes Item nicht dem, bei der restlichen Itembatterie beobachteten Antwortmuster, so wird das als Indiz dafür gewertet, dass dieses Item die Zieldimension nicht ausreichend erfassen konnte.

\subsection{Prüfung der Güte der Items zur Erhebung der Ausprägungen der laten- ten Variablen „Synergiesystem“}

Zur Erhebung der Ausprägungen der latenten Variablen „Synergiesystem“ wurden zwölf Items in den Fragebogen integriert. Um die Güte dieser zwölf Items zu prüfen wurden aus den erhobenen Daten mit dem Softwarepaket SPSS 10 Trennschärfekoeffizienten berechnet. Die Ergebnisse sind in Abbildung 30 zusammengefasst:

Abb. 30: Trennschärfekoeffizienten der Itembatterie „Synergiesystem“

\begin{tabular}{|l|l|l|l|}
\hline $\begin{array}{l}\text { Item } \\
\text { Nr. }^{317}\end{array}$ & Formulierung des Items im Fragebogen & $\mathbf{r}$ & Bewertung \\
\hline 4 & Die Kooperation ist seit ihrem Bestand intensiver geworden. & 0,663 & geeignet \\
\hline 5 & Ich bin in dieser Kooperation rechtlich selbständig. & 0,462 & geeignet \\
\hline 6 & $\begin{array}{l}\text { Ich stimme mein Verhalten mit meinem Kooperationspartner } \\
\text { ab. }\end{array}$ & 0,600 & geeignet \\
\hline
\end{tabular}

316 Beim Konsistenztest mittels Trennschärfekoeffizienten wird unterstellt, dass die Mehrzahl der Items die Zieldimension in hohem Maße anspricht. "Der Konsistenztest in Form der Berechnung von Trennschärfekoeffizienten unterstellt quasi, dass man sich in der Summe weniger irrt, als bei der Formulierung der Einzelitems." (Diekmann 2002, 212)

317 Die Itemnummer bezieht sich auf die Reihenfolge der Fragen im Fragebogen. Der Bezug zwischen den Fragen im Fragebogen und den Dimensionen ist in der Verbindungstabelle (Abb. 27) dargestellt. Dort ist auch ersichtlich, dass einige Dimensionen mit mehr als einem Item erhoben wurden. 


\begin{tabular}{|l|l|l|l|}
\hline 7 & $\begin{array}{l}\text { Ich denke mein Kooperationspartner und ich haben gleich } \\
\text { starkes Interesse an der Kooperation. }\end{array}$ & 0,703 & geeignet \\
\hline 8 & Ich kann die Kooperation jederzeit einseitig beenden. & 0,425 & geeignet \\
\hline 9 & $\begin{array}{l}\text { Mein Kooperationspartner und ich gehen in bestimmten Berei- } \\
\text { chen gemeinsam vor. }\end{array}$ & 0,666 & geeignet \\
\hline 10 & $\begin{array}{l}\text { Ich könnte durch egoistisches Verhalten meinem Koopera- } \\
\text { tionspartner Schaden zufügen. }\end{array}$ & 0,454 & geeignet \\
\hline 19 & $\begin{array}{l}\text { Ich möchte durch die Kooperation möglichst schnell Erfolg } \\
\text { erzielen. }\end{array}$ & 0,455 & geeignet \\
\hline 30 & $\begin{array}{l}\text { Bei Entscheidungen die die Kooperation betreffen, hat die } \\
\text { Stimme jedes Kooperationspartners gleich viel Gewicht. }\end{array}$ & 0,622 & geeignet \\
\hline 32 & $\begin{array}{l}\text { Entscheidungen die die Kooperation betreffen, werden aus- } \\
\text { schließlich von meinem Kooperationspartner und mir getrof- } \\
\text { fen. Er gibt keinen Einfluss von außen. }\end{array}$ & 0,283 & nicht \\
\hline 36 & $\begin{array}{l}\text { Mein Kooperationspartner und ich sprechen über die Koope- } \\
\text { ration. }\end{array}$ & 0,391 & geeignet \\
\hline 38 & $\begin{array}{l}\text { Mein Kooperationspartner könnte mir durch egoistisches Ver- } \\
\text { halten Schaden zufügen. }\end{array}$ & 0,449 & geeignet \\
\hline
\end{tabular}

Quelle: Eigene Darstellung

Es zeigt sich, dass die Werte des Items Nummer 32 deutlich schwächer mit den Summenscores korrelieren als die restlichen Items der Itembatterie „Synergiesystem“. Scheinbar konnte die Formulierung dieses Items die Dimension „Flexibilität/Unabhängigkeit“ nur ungenügend erfassen. Das Statement, wonach es bei Entscheidungen der Kooperationspartner, die die Kooperation betreffen, keinen Einfluss von außen gibt, könnte von den Befragten missinterpretiert worden sein. Es ist denkbar, dass die Befragten das Statement als Negierung entscheidungsrelevanter Einflussfaktoren der Unternehmensumwelt bzw. der Kooperationssystemumwelt aufgefasst haben.

Zur Verdeutlichung werden die Ergebnisse des Konsistenztests zusätzlich graphisch dargestellt. Abbildung 31 zeigt die Items der Itembatterie „Synergiesystem“, die latente Variable „Synergiesystem“ sowie die, aus den Daten berechneten, Korrelationen zwischen den Items untereinander und der latenten Variablen. Die Korrelationen werden in der Grafik als Pfeile dargestellt und sind nach vier Dimensionen bestimmt: Die im konkreten Fall betrachtete Richtung der Beziehung wird durch die Ausrichtung des Pfeilkopfs ausgedrückt. Die Stärke der Korrelation bestimmt die Stärke des Pfeils. Die Farbe des Pfeils gibt Auskunft über die Irrtumswahrscheinlichkeit mit der der jeweilige Trennschärfekoeffizient behaftet ist. Das im Pfeilkopf dargestellte Vorzeichen informiert den Leser, ob es 
sich bei der dargestellten Beziehung um einen positiven oder negativen Zusammenhang handelt. Die Kodifizierung im Detail ist der Legende zu entnehmen.

Abb. 31: Güte der Itembatterie „Synergiesystem“

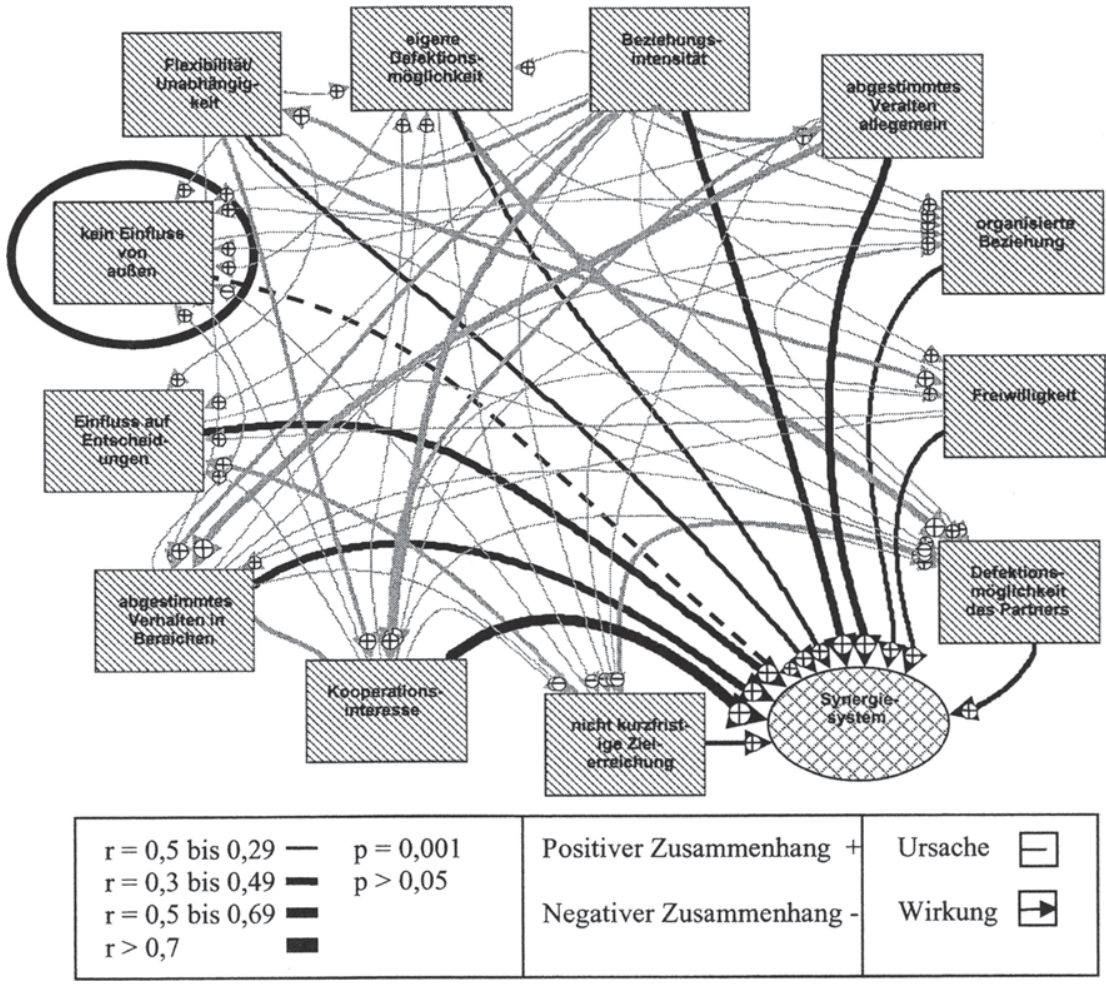

Quelle: Eigene Darstellung

Aus der Grafik geht deutlich hervor, dass alle Items außer dem Item „kein Einfluss von außen“ stark auf die latente Variable „Synergiesystem“ laden. Sie alle sind zumindest mit Pfeilen der Stärke zwei mit der latenten Variablen (Zieldimension) verbunden. Von dem, in der Grafik rot markierten Item Nummer 32 geht hingegen nur ein dünner schwarzer Pfeil in Richtung der latenten Variablen aus. Das bedeutet, dass die Werte, die mit diesem Item erhoben wurden, nur schwach mit den Summenscores der Itembatterie „Synergiesystem“ korrelieren. Dieses Item kann daher als wenig geeignet eingestuft werden, die Ausprägungen der Zieldimension zu erheben. 
5.7.1.1.2. Prüfung der Güte der Items zur Erhebung der Ausprägungen der latenten Variablen „Selbstverpflichtung“

Berechnet man Trennschärfekoeffizienten für die zehn Items der Itembatterie „Selbstverpflichtung“ so erhält man folgendes Ergebnis:

Abb. 32: Trennschärfekoeffizienten der Itembatterie ..Selbstverpflichtung“

\begin{tabular}{|l|l|c|l|}
\hline $\begin{array}{c}\text { Item } \\
\text { Nr. }\end{array}$ & Formulierung des Items im Fragebogen & $\mathbf{r}$ & Bewertung \\
\hline 2 & Diese Kooperation ist meine erste Kooperation. & 0,572 & geeignet \\
\hline 3 & $\begin{array}{l}\text { Ich habe mit meinem jetzigen Kooperationspartner schon früher } \\
\text { einmal kooperiert. }\end{array}$ & 0,432 & geeignet \\
\hline 11 & $\begin{array}{l}\text { Ich habe vor der Kooperation Gutes über meinen jetzigen } \\
\text { Kooperationspartner gehört. }\end{array}$ & 0,464 & geeignet \\
\hline 12 & $\begin{array}{l}\text { Die Kooperation hat einen großen Einfluss auf den Erfolg } \\
\text { meines Unternehmens. }\end{array}$ & 0,331 & geeignet \\
\hline 13 & $\begin{array}{l}\text { Ich habe vor der Kooperation Gutes über die Branche gehört, in } \\
\text { der mein jetziger Kooperationspartner tätig ist. }\end{array}$ & 0,400 & geeignet \\
\hline 14 & $\begin{array}{l}\text { Ich habe mich vor der Kooperation über meinen Kooperations- } \\
\text { partner informiert. }\end{array}$ & 0,501 & geeignet \\
\hline 15 & $\begin{array}{l}\text { Jede Kooperation stellt für die beteiligten Unternehmen ein } \\
\text { Risiko dar. }\end{array}$ & 0,235 & $\begin{array}{l}\text { nicht } \\
\text { geeignet }\end{array}$ \\
\hline 16 & Ich bin überzeugt Rückschläge verkraften zu können. & 0,544 & geeignet \\
\hline 18 & $\begin{array}{l}\text { Damit eine Kooperation gelingt, muss man seinem Koopera- } \\
\text { tionspartner einen Vertauensvorschuss geben, auch wenn man } \\
\text { damit ein Risiko eingeht. }\end{array}$ & 0,489 & geeignet \\
\hline 20 & Ich bin bereit ein Risiko einzugehen. & 0,462 & geeignet \\
\hline
\end{tabular}

Quelle: Eigene Darstellung

Wie aus Abbildung 32 hervorgeht, korrelieren alle Items deutlich stärker mit dem Summenscore als Item Nummer 15. Dieses Item, das darauf abzielte das Risikobewusstsein des Befragten zu erheben, konnte diesen Aspekt der Dimension „Risikobereitschaft/eingegangenes Risiko“ nicht erfassen. Vermutlich war die Formulierung dieses Items zu allgemein gefasst, um einen Beitrag zur Erhebung der Ausprägungen der latenten Variablen „Selbstverpflichtung“ leisten zu können.

Die geringe Eignung des Items Nummer 15 zeigt sich auch deutlich in der graphischen Darstellung. Nur ein dünner Pfeil verbindet das Item 15 „Jede Kooperation stellt ein Risiko dar“ mit der latenten Variablen "Synergiesystem“ (Zieldimension). 
Abb. 33: Güte der Itembatterie "Selbstverpflichtung"

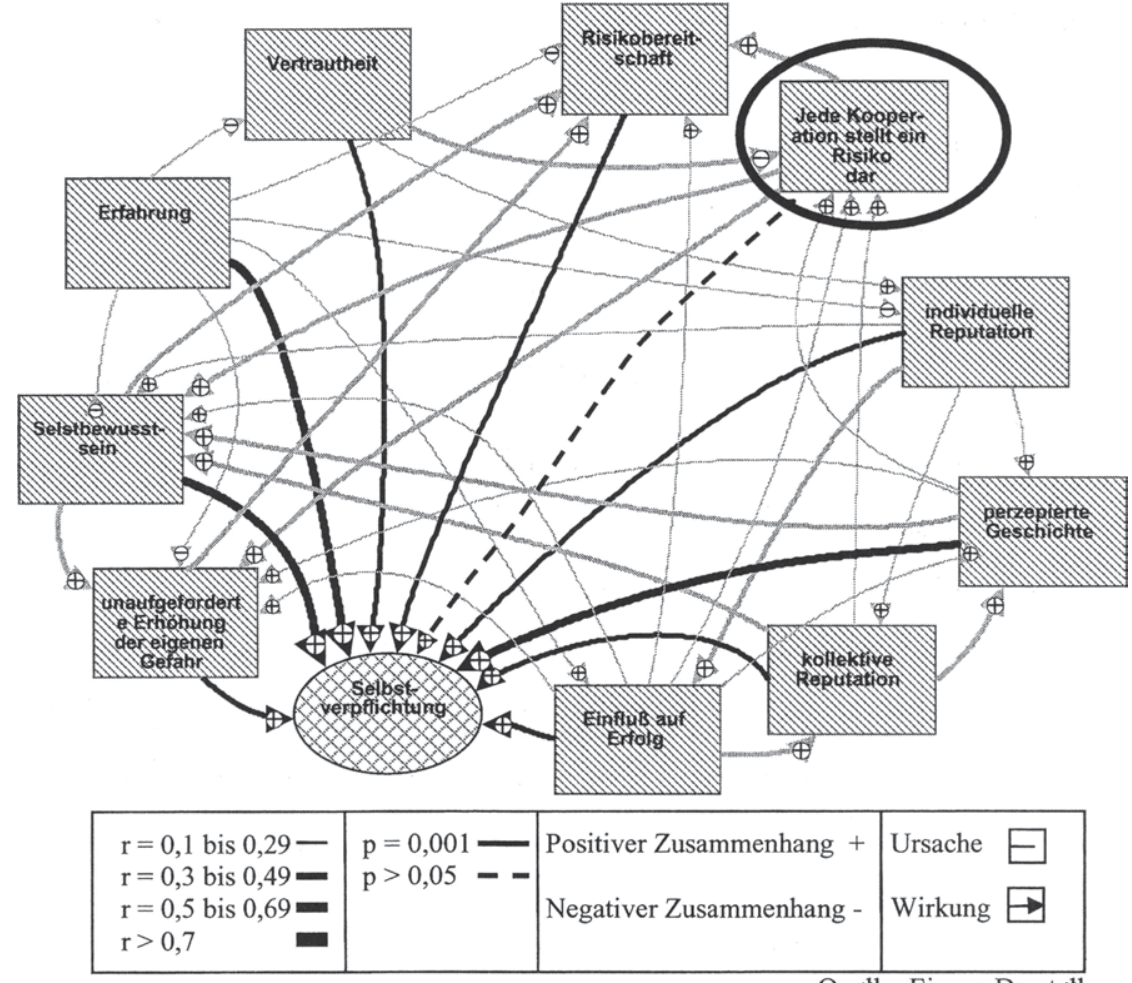

Qualle: Eigene Darstedlung

5.7.1.1.3. Prüfung der Güte der Items zur Erhebung der Ausprägungen der latenten Variablen „Erfolg aus der Beziehungsperspektive“"

Die Items der Itembatterie „Erfolg aus der Beziehungsperspektive“ erwiesen sich zum weitaus überwiegenden Teil als geeignet, die Ausprägungen der Zieldimension zu erfassen. Nur bei zwei Items ergab die Berechnung der Trennschärfekoeffizienten ein gegenteiliges Ergebnis. Sehr gering war die Korrelation zwischen dem Item Nummer 31 und dem relevanten Summenscore. Auch bei Item Nummer 33 folgten die Antworten nicht dem bei den restlichen Items innerhalb dieser Itembatterie beobachteten Antwortmuster. Diese zwei Items konnten wenig zur Erhebung der latenten Variablen „Erfolg aus der Beziehungsperspektive“ beitragen. Abbildung 34 fasst die Ergebnisse der Konsistenzprüfung der Itembatterie „Erfolg aus der Beziehungsperspektive“ zusammen. 
Abb. 34: Trennschärfekoeffizienten der Itembatterie „Erfolg aus der Beziehungsperspektive“

\begin{tabular}{|l|l|l|l|}
\hline $\begin{array}{c}\text { Item } \\
\text { Nr. }\end{array}$ & Formulierung des Items im Fragebogen & r & Bewertung \\
\hline 17 & $\begin{array}{l}\text { Ich kann meinen Kooperationspartner direkt auf das an- } \\
\text { sprechen, was mir am Herzen liegt. }\end{array}$ & 0,636 & geeignet \\
\hline 25 & $\begin{array}{l}\text { Diskussionen mit meinem Kooperationspartner führen immer } \\
\text { zu einem Ergebnis. }\end{array}$ & 0,431 & geeignet \\
\hline 26 & Ich habe auch privat Kontakt zu meinem Kooperationspartner. & 0,384 & geeignet \\
\hline 27 & Mein Kooperationspartner ist ein komplizierter Mensch. & 0,617 & geeignet \\
\hline 28 & $\begin{array}{l}\text { Ich kenne die Abläufe im Unternehmen meines Koopera- } \\
\text { tionspartners. }\end{array}$ & 0,568 & geeignet \\
\hline 29 & $\begin{array}{l}\text { Auf Nachfrage bekomme ich immer die relevanten Infor- } \\
\text { mationen über die Abläufe im Unternehmen meines Koope- } \\
\text { rationspartners. }\end{array}$ & 0,523 & geeignet \\
\hline 31 & $\begin{array}{l}\text { Bei Entscheidungen habe ich einen größeren Einfluss auf das } \\
\text { Ergebnis als mein Kooperationspartner. }\end{array}$ & 0,139 & $\begin{array}{l}\text { nicht } \\
\text { geeignet }\end{array}$ \\
\hline 33 & $\begin{array}{l}\text { Mein Kooperationspartner ist ein großer Taktiker. } \\
\text { Mein Kooperationspartner ist für mich stets erreichbar. }\end{array}$ & 0,284 & $\begin{array}{l}\text { nicht } \\
\text { geeignet }\end{array}$ \\
\hline 37 & $\begin{array}{l}\text { Ich kann mit meinem Kooperationspartner offen über Prob- } \\
\text { leme sprechen. }\end{array}$ & 0,770 & geeignet \\
\hline
\end{tabular}

Quelle: Eigene Darstellung

In Abbildung 35 sind es dann auch das Item Nummer 31 und das Item Nummer 33, die nur mit einem dünnen Pfeil mit der Zieldimension „Erfolg aus der Beziehungsperspektive" verbunden sind. Die restlichen Items dieser Itembatterie korrelieren stark mit der Zieldimension. Sie sind in der Abbildung 35 daher auch mit einem dicken Pfeil mit der latenten Variablen „Erfolg aus der Beziehungsperspektive" verbunden.

Es wird deutlich, dass diese zwei Items weit weniger bzw. gar nicht geeignet waren die Ausprägungen der latenten Variablen zu erfassen. Vermutlich war Item Nummer $33 \mathrm{zu}$ indirekt formuliert. Der Begriff „Taktiker“ könnte von den Befragten eher mit der Fähigkeit zu strategischem Vorgehen in Verbindung gebracht worden sein, als mit taktischem Verhalten, wie bei der Fragebogenkonstruktion unterstellt wurde. So wurde dieses Item missinterpretiert und das Antwortverhalten verzerrt. Item Nummer 31 war scheinbar zu allgemein formuliert. Der Geltungsbereich des Statements hätte auf Entscheidungen, die die Kooperation betreffen, eingeschränkt werden sollen. 
Abb. 35: Güte der Itembatterie „Erfolg aus der Beziehungsperspektive“

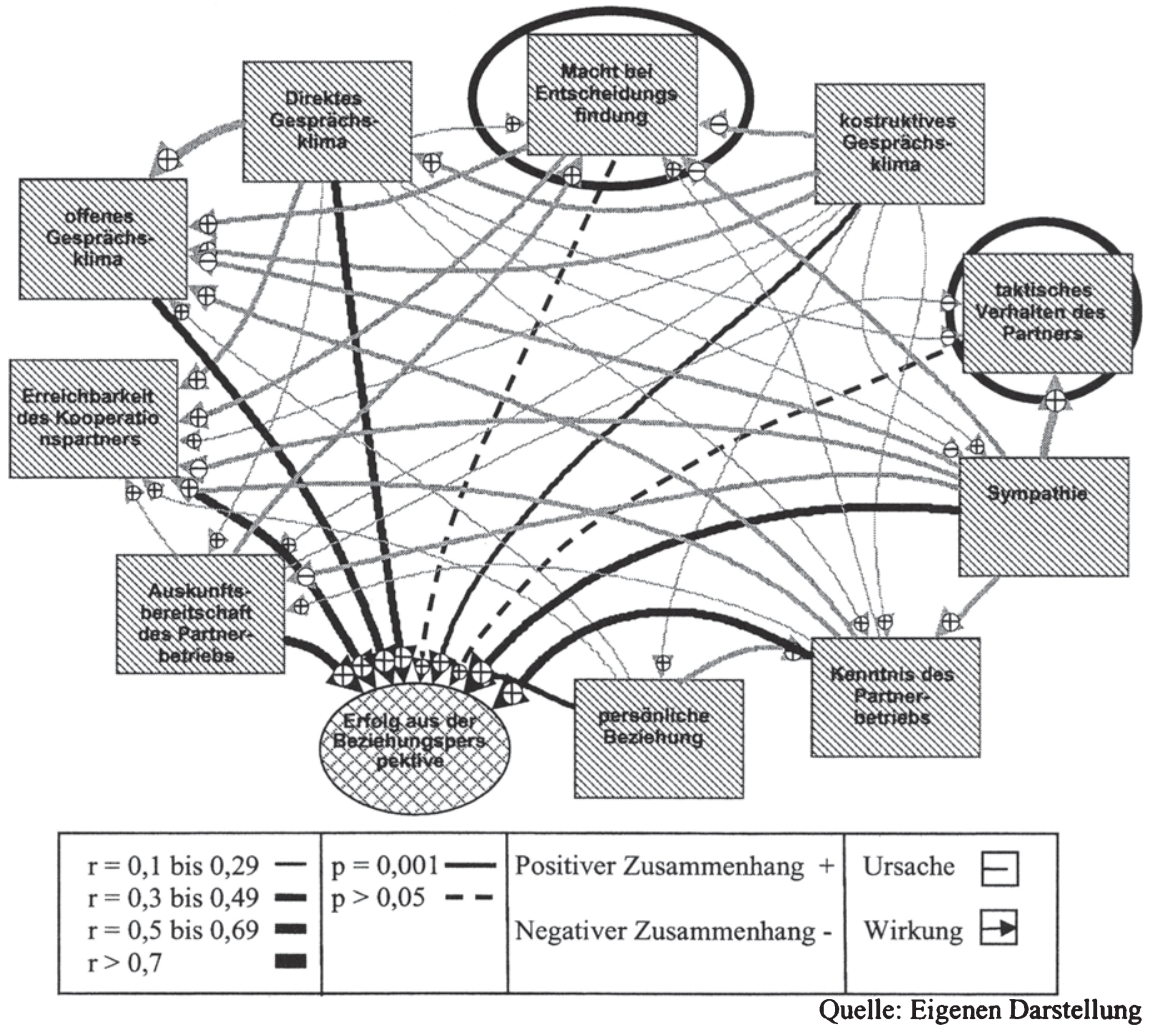

5.7.1.1.4. Prüfung der Güte der Items zur Erhebung der Ausprägungen der latenten Variablen „Erfolg aus der Binnenperspektive“

Die Items der Itembatterie „Erfolg aus der Binnenperspektive“ teilen sich in zwei Gruppen. Die erste Gruppe umfasst jene Items, die sich an alle Befragten richten. Die zweite Gruppe bilden jene Items, die sich nur an Befragte richten, die zum Zeitpunkt der Befragung eine Kooperation mit einem anderen Unternehmen unterhielten. Die Berechnung der Trennschärfekoeffizienten zeigt, dass diese zwei Gruppen nicht die selbe Dimension erfassen. Während die Items der ersten Gruppe bei den Befragten ein konsistentes Antwortverhalten provozierten, entsprachen die Antworten auf die Items der zweiten Gruppe nicht diesem Antwortmuster. Sie waren für die Erhebung der Ausprägungen der latenten Variablen (Zieldimension) nur wenig geeignet. 
Abb. 36: Trennschärfekoeffizienten der Itembatterie „Erfolg aus der Binnenperspektive“

\begin{tabular}{|l|l|c|l|}
\hline $\begin{array}{c}\text { Item } \\
\text { Nr. }\end{array}$ & Formulierung des Items im Fragebogen & r & Bewertung \\
\hline 24 & $\begin{array}{l}\text { Seit Bestand der Kooperation haben weniger Mitarbeiter mein } \\
\text { Unternehmen verlassen als zuvor. }\end{array}$ & 0,260 & $\begin{array}{l}\text { nicht } \\
\text { geeignet }\end{array}$ \\
\hline 35 & $\begin{array}{l}\text { eit Beginn der Kooperation hat sich die Qualifikation meiner } \\
\text { Mitarbeiter erhöht. }\end{array}$ & $-0,067$ & $\begin{array}{l}\text { nicht } \\
\text { geeignet }\end{array}$ \\
\hline 41 & $\begin{array}{l}\text { Die Prognosen meines Unternehmens über den Geschäfts- } \\
\text { verlauf sind verlässlich. }\end{array}$ & 0,528 & geeignet \\
\hline 48 & $\begin{array}{l}\text { Die Qualifikation meiner Mitarbeiter konnte in den letzten drei } \\
\text { Jahren gesteigert werden. }\end{array}$ & 0,477 & geeignet \\
\hline 49 & $\begin{array}{l}\text { In den letzten drei Jahren haben mehr Mitarbeiter mein Unter- } \\
\text { nehmen verlassen als zuvor. }\end{array}$ & 0,578 & geeignet \\
\hline
\end{tabular}

Quelle: Eigene Darstellung

Sowohl die Werte in Abbildung 36 als auch die graphische Darstellung weiter unten machen deutlich, dass diese Itembatterie nicht auf eine, sondern auf zwei Zieldimensionen ausgerichtet war. Vermutlich war der Versuch, die latente Variable sowohl in Bezug auf die Bestandsdauer der Kooperation, als auch in Bezug auf die Zeitspanne der letzten drei Jahre zu erfassen, zu ambitioniert. Es ist trotz ähnlicher Formulierung der jeweiligen Itempaare ${ }^{318}$ nicht gelungen, den Befragten den inhaltlichen Zusammenhang der Items zu kommunizieren.

Da die Antworten auf die zwei Items, die den Erfolg aus der Binnenperspektive auf die Zeitspanne der letzten drei Jahre beziehen, dem Antwortmuster des Items Nummer 41 folgen, wird die latente Variable „Erfolg aus der Binnenperspektive“ in Bezug auf die Zeitspanne der letzten drei Jahre als Bezugspunkt für den Konsistenztest gewählt. Die Werte, die für das Item Nummer 24 „Mitarbeiterfluktuation bei Kooperationen“ und das Item Nummer 35 „Mitarbeiterqualifikation bei Kooperationen“ erhoben wurden, korrelieren nur in sehr geringem Ausmaß mit der Zieldimension. Folglich werden die zwei Items (Item Nummer 24 und Item Nummer 35) als wenig geeignet identifiziert, um die Ausprägungen der latenten Variablen „Erfolg aus der Binnenperspektive“ zu erheben.

318 Als Itempaar werden in der Folge jeweils die zwei Items bezeichnet, die auf die Erfassung der selben Dimension abzielen, die zu erfassende Dimension jedoch mit verschiedenen Zeitspannen ("Bestandsdauer der Kooperation" oder "in den letzten drei Jahren") in Beziehung setzen. 
Abb. 37: Güte der Itembatterie „Erfolg aus der Binnenperspektive“

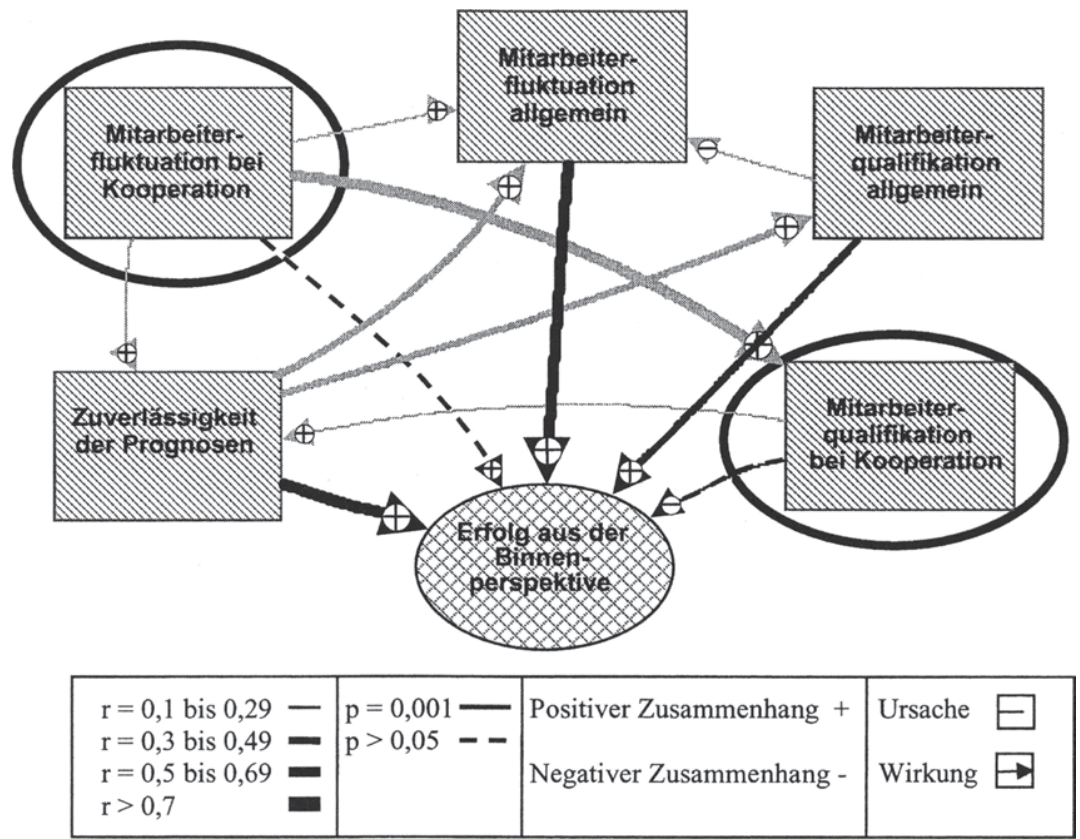

Quelle: Eigene Darstellung

5.7.1.1.5. Prüfung der Güte der Items zur Erhebung der Ausprägungen der latenten Variablen „Erfolg aus der Außenperspektive“"

Die Itembatterie zur Erhebung der Ausprägungen der latenten Variablen „Erfolg aus der Außenperspektive“ besteht aus sechs Items. Der Konsistenztest auf der Basis der Berechnung von Trennschärfekoeffizienten ergibt die in Abbildung 38 zusammen-gefassten Ergebnisse:

Abb. 38: Trennschärfekoeffizienten der Itembatterie „Erfolg aus der Außenperspektive“

\begin{tabular}{|l|l|c|l|}
\hline $\begin{array}{c}\text { Item } \\
\text { Nr. }\end{array}$ & Formulierung des Items im Fragebogen & r & Bewertung \\
\hline 39 & $\begin{array}{l}\text { Ich habe seit Bestand der Kooperation meinen Marktanteil ver- } \\
\text { größert. }\end{array}$ & 0,591 & geeignet \\
\hline 40 & $\begin{array}{l}\text { Meine Kunden sind stets mit den Leistungen meines Unter- } \\
\text { nehmens zufrieden. }\end{array}$ & 0,545 & geeignet \\
\hline 44 & $\begin{array}{l}\text { Ich konnte in den letzten drei Jahren meinen Marktanteil } \\
\text { deutlich vergrößern. }\end{array}$ & 0,647 & geeignet \\
\hline
\end{tabular}




\begin{tabular}{|l|l|l|l|}
\hline 45 & $\begin{array}{l}\text { In den letzten drei Jahren ist es schwieriger geworden, bei } \\
\text { Angeboten den Zuschlag zu bekommen. }\end{array}$ & 0,340 & geeignet \\
\hline 46 & Die meisten meiner Kunden sind Stammkunden. & 0,726 & geeignet \\
\hline 47 & Die meisten meiner Lieferanten sind Stammlieferanten. & 0,782 & geeignet \\
\hline
\end{tabular}

Quelle: Eigene Darstellung

Wie aus der Tabelle ersichtlich, korrelieren alle Items relativ stark mit den Summenscores. Dies ist insofern überraschend, als auch bei dieser Itembatterie, so wie bei der Itembatterie zu Messung der Ausprägungen der latenten Variablen „Erfolg aus der Binnenperspektive", versucht wurde, die Zieldimension einerseits in Bezug auf die Bestandsdauer der Kooperation und andererseits in Bezug auf die Zeitspanne der letzten drei Jahre zu erheben. Während dieser Versuch im Fall der latenten Variablen „Erfolg aus der Binnenperspektive“ gescheitert ist, konnte bei der Itembatterie zur Erfassung der latenten Variablen „Erfolg aus der Außenperspektive" ein durchgehendes Antwortmuster beobachtet werden. Es ist anscheinend bei dieser Itembatterie eher gelungen, den Befragten den inhaltlichen Zusammenhang der Itempaare zu kommunizieren.

Abb. 39: Güte der Itembatterie „Erfolg aus der Außenperspektive“

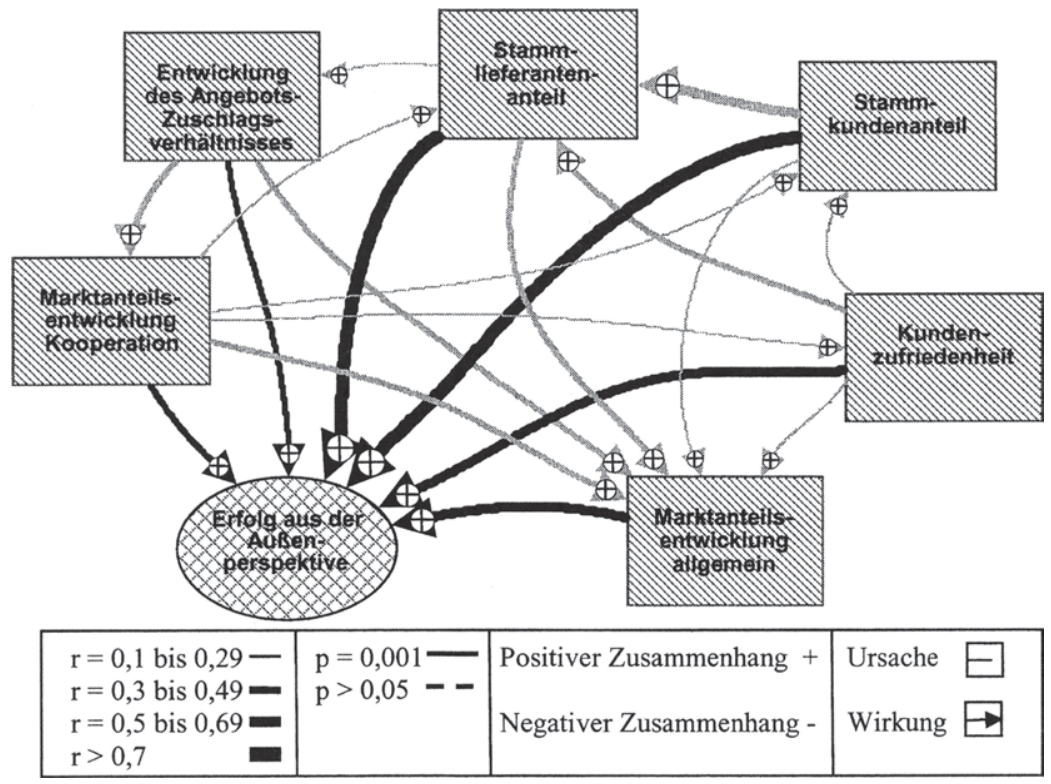

Quelle: Eigene Darstellung 
Abbildung 39 verdeutlicht dieses positive Ergebnis der Konsistenzprüfung der Items der Itembatterie „Erfolg aus der Außenperspektive“. Alle Items sind mit der Zieldimension mindestens mit einem Pfeil der Stärke zwei verbunden. Das bedeutet, dass alle Items relativ stark auf die latente Variable „Erfolg aus der Außenperspektive“ laden. Sie alle konnten einen Teil der Zieldimension erfassen. Alle Items waren für die Erhebung der Ausprägungen der latenten Variablen „Erfolg aus der Außenperspektive" geeignet.

\subsection{Prüfung der Güte der Items zur Erhebung der Ausprägungen der latenten Variablen „Erfolg aus der Finanzperspektive“}

Die letzten sechs noch zu prüfenden Items sind in der Itembatterie „Erfolg aus der Finanzperspektive“ zusammengefasst. Der Konsistenztest für diese Items ergibt folgende Trennschärfekoeffizienten:

Abb. 40: Trennschärfekoeffizienten der Itembatterie „Erfolg aus der Finanzperspektive“

\begin{tabular}{|l|l|c|l|}
\hline $\begin{array}{c}\text { Item } \\
\mathbf{N r}\end{array}$ & Formulierung des Items im Fragebogen & $\mathbf{r}$ & Bewertung \\
\hline 21 & $\begin{array}{l}\text { Seit die Kooperation besteht, habe ich verstärkt in mein Unter- } \\
\text { nehmen investiert. }\end{array}$ & 0,511 & geeignet \\
\hline 22 & $\begin{array}{l}\text { Ich konnte seit Beginn der Kooperation meinen Umsatz } \\
\text { deutlich steigern. }\end{array}$ & 0,515 & geeignet \\
\hline 23 & $\begin{array}{l}\text { Ich konnte seit Beginn der Kooperation meinen Cash-Flow } \\
\text { deutlich steigern. }\end{array}$ & 0,511 & geeignet \\
\hline 42 & $\begin{array}{l}\text { Ich konnte in den letzten drei Jahren meinen Cash-Flow } \\
\text { deutlich steigern. }\end{array}$ & 0,675 & geeignet \\
\hline 43 & $\begin{array}{l}\text { Ich konnte in den letzten drei Jahren meinen Umsatz deutlich } \\
\text { steigern. }\end{array}$ & 0,769 & geeignet \\
\hline 50 & $\begin{array}{l}\text { Ich habe in den letzten drei Jahren mehr (Geld) in mein Unter- } \\
\text { nehmen investiert als zuvor. }\end{array}$ & 0,655 & geeignet \\
\hline
\end{tabular}

Quelle: Eigene Darstellung

Das Ergebnis des Konsistenzanalyse zeigt, dass sämtliche Items der Itembatterie „Erfolg aus der Finanzperspektive“ in hohem Maße mit den Summenscores korrelieren. Die Berechnung der Trennschärfekoeffizienten ergibt durchwegs Werte über 0,5. Das bedeutet, dass alle in dieser Itembatterie enthaltenen Items zur Erfassung der latenten Variablen „Erfolg aus der Finanzperspektive“ beitragen konnten. Auch bei dieser Itembatterie ist es gelungen Ausprägungen der latenten Variablen einerseits in Bezug auf die Bestandsdauer der Kooperation und andererseits in Bezug auf die Zeitspanne der letzten drei Jahre zu erheben. 
Abb. 41: Güte der Itembatterie „Erfolg aus der Finanzperspektive“

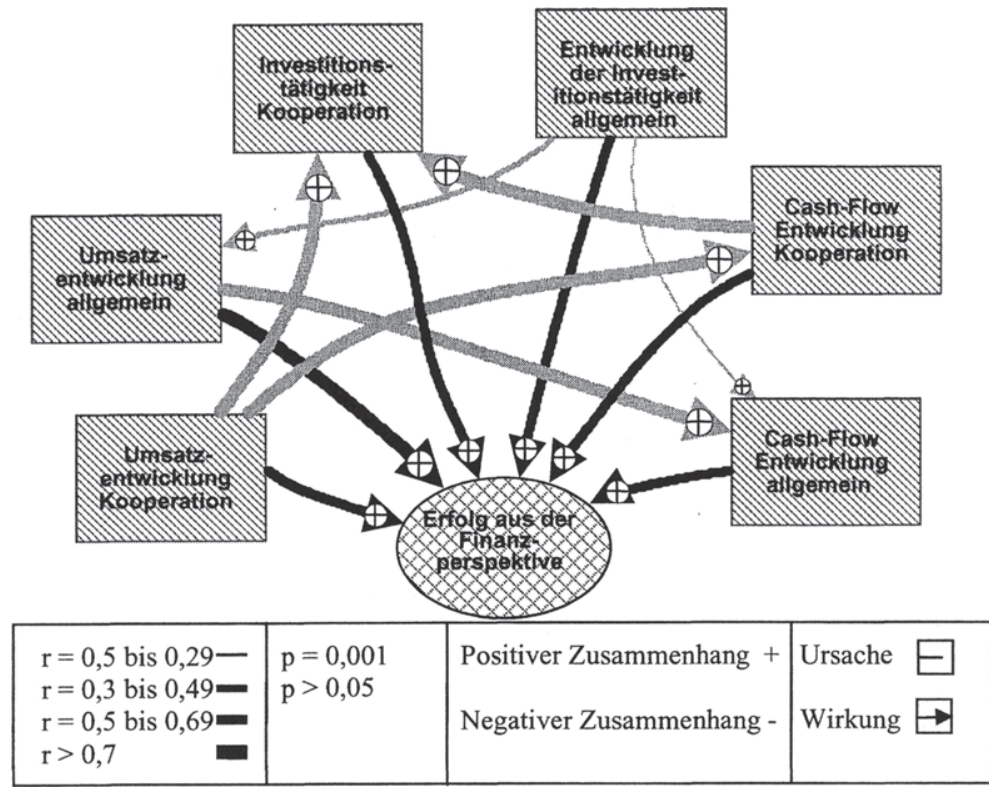

Quelle: Eigene Darstellung

Das über alle Items konsistente Antwortverhalten der Befragten geht auch deutlich aus der graphischen Darstellung hervor. Sämtliche Items sind zumindest mit einem Pfeil der Stärke drei mit der Zieldimension verbunden. Sie alle waren für die Erhebung der Ausprägungen der latenten Variablen „Erfolg aus der Finanzperspektive geeignet.

\subsubsection{Test auf Reliabilität mittels der Split-half Methode}

Nachdem im vorhergehenden Abschnitt die Güte der einzelnen Items untersucht wurde, wird an dieser Stelle nun geprüft, ob die Itembatterien des Erhebungsinstruments auch tatsächlich verlässlich waren. Das Kriterium der Verlässlichkeit (Reliabilität) fordert Reproduzierbarkeit von Testergebnissen. Da bei der Befragung im Rahmen des vorliegenden Projekts die Messung mit multiplen Indikatoren durchgeführt wurde, kann mit der Split-half-Methode nach Gutmann das Vorliegen zweier Erhebungsinstrumente simuliert werden. Dabei werden die Items jeder Itembatterie auf zwei Gruppen aufgeteilt. Die so gebildeten Itemgruppen werden als getrennte Erhebungsinstrumente behandelt. Vereinfacht ausgedrückt kann die Reliabilität des ursprünglichen Erhebungsinstruments dann aus 
der Korrelation der beiden Testhälften erhobenen werden. ${ }^{319}$ Aus der Halbierung folgt jedoch eine Unterschätzung der Korrelation zwischen den beiden Testhälften, da die Korrelation mit steigender Anzahl an Indikatoren anwächst. Mit der Spearman-Brown-Formel kann diese Verzerrung rechnerisch kompensiert werden. In Abbildung 42 sind für jede Itembatterie sowohl der originäre Wert nach Anwendung der Split-half-Methode, als auch der mit der Spearman-BrownFormel korrigierte Wert dargestellt.

Abb. 42: Ergebnisse des Reliabilitätstests

\begin{tabular}{|c|c|c|c|c|c|c|}
\hline & 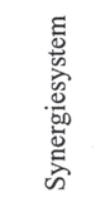 & 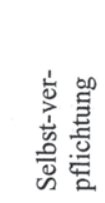 & 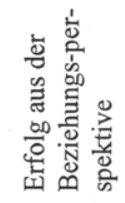 & 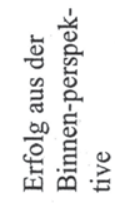 & 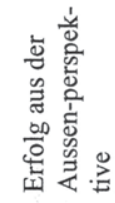 & 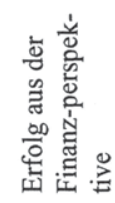 \\
\hline Gutmann Split-half & 0,5055 & 0,4315 & 0,4497 & 0,3960 & 0,4545 & 0,8254 \\
\hline Spearman-Brown & 0,5075 & 0,4871 & 0,4497 & 0,4749 & 0,5169 & 0,8890 \\
\hline
\end{tabular}

Quelle: Eigene Darstellung

Es zeigt sich, dass die Korrektur nach Spearman-Brown wie erwartet vor allem bei den kurzen Itembatterien deutliche Differenzen zu den originären Werten ergibt. Bedenkt man jedoch, dass bei dieser Untersuchung versucht wurde, abstrakte Phänomene durch schrittweise Operrationalisierung messbar zu machen (siehe Abbildung 9), so verwundern die eher bescheidenen Ergebnisse des Reliabitätstests nicht. Außerdem ist die Reliabilität in großem Ausmaß von den Interkorrelationen und damit auch von den Trennschärfekorrelationen abhängig. ${ }^{320}$ Somit ist die eher bescheidene Reliabilität bei Einstellungsmessungen weitgehend auf die typisch niedrigen Trennschärfekoeffizienten der Einzelitems bei solchen Messungen zurückzuführen - ein allgemeines Problem der Anwendung quantitativer Methoden auf solche Forschungsobjekte. Dennoch kann auch in den Sozialwissenschaften auf quantitative Untersuchungen und die aus ihnen gewonnenen Erkenntnisse nicht verzichtet werden. Vielmehr ist darauf zu achten, dass die Unzulänglichkeiten der quantitativen Forschungsmethode nicht unreflektiert bleiben. Ist ein verantwortungsvoller Umgang mit den angewandten Methoden und den gewonnenen Ergebnissen gewährleistet, so leisten solche Untersuchungen einen wertvollen Beitrag auch zum Verständnis abstrakter Phänomene. 


\subsection{2. ÜBERPRÜFUNG DER GÜLTIGKEIT DES THEORETISCHEN MODELLS}

Die Prüfung des Erhebungsinstrumentes hat ergeben, dass von einigen Einzelitems abgesehen, die Messung die geforderten Gütekriterien hinreichend erfüllt, um auf der erhobenen Datenbasis starke Aussagen über Strukturen treffen zu können. Eine Analyse der Einzelbeziehungen wäre auch auf Grund der Komplexität des Forschungsobjektes nicht realistisch. In Abbildung 43 sind zur Veranschaulichung sämtliche Messvariablen sowie die zwischen ihnen erhobenen Korrelationen dargestellt. ${ }^{321}$

Abb. 43: Die Komplexität des Forschungsgegenstandes

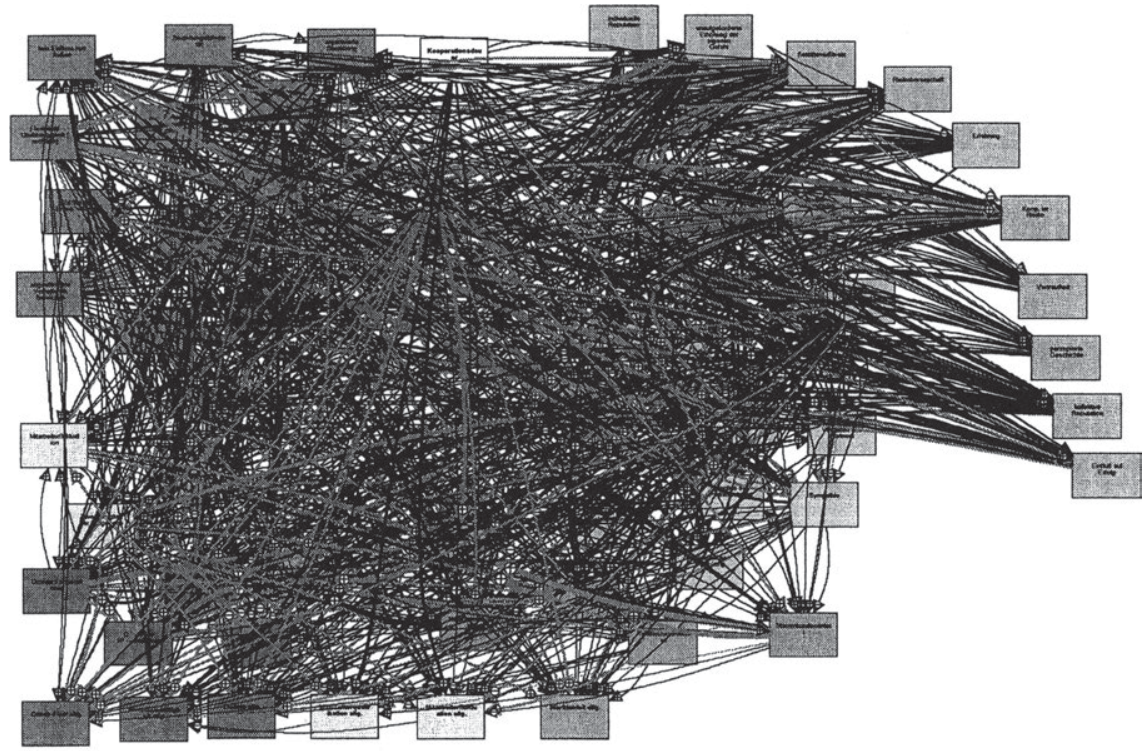

$$
\begin{aligned}
& \mathrm{r}=0,5 \text { bis } 0,29-\mathrm{p}=0,001 \text { 圆 Positiver Zusammenhang }+ \text { Ursache } E \\
& \mathrm{r}=0,3 \text { bis } 0,49-\mathrm{p}=0,05 \\
& \mathrm{r}=0,5 \text { bis } 0,69=\mathrm{p}>0,05 \square \quad \text { Negativer Zusammenhang - Wirkung } \leftrightarrow \\
& r>0,7
\end{aligned}
$$

Quelle: Eigene Darstellung

321 In der Grafik sind sämtliche 1250 Beziehungen eingetragen, die zwischen den Messvariablen bestehen. Selbst, wenn man nur die hoch signifikanten Zusammenhänge herausfiltert, würde eine unüberschaubare Menge an Relationen übrig bleiben, deren Analyse auf der Basis von Einzelbeziehungen nicht sinnvoll durchführbar wäre. 
Die große Anzahl an erhobenen Beziehungen zwingt zur Komplexitätsreduktion. Dies entspricht auch dem Ansatz der vorliegenden empirischen Erhebung, die angetreten ist, die in der theoretischen Argumentation behaupteten, grundlegenden Kausalstrukturen mit der Empirie zu konfrontieren. Weder bei der Konzeption der Erhebung, noch bei der Datenauswertung liegt der Fokus auf dem Studium von Einzelaspekten, sondern auf dem Erkennen von groben Strukturen innerhalb der einzelnen latenten Variablen und den latenten Variablen untereinander. Es geht um Mustererkennung!

Im Einzelnen ist zu analysieren, inwieweit sich die, zur Erfassung der latenten Variablen formulierten Messmodelle, anhand der Daten bestätigen lassen. Es handelt sich dabei nicht um eine Entscheidungsfrage, sondern um eine graduelle Bewertung des Fits zwischen dem theoretisch formulierten Messmodell und dem aus den korrespondierenden empirischen Daten erkennbaren Mustern.

Im Folgenden werden die Messmodelle den in den Daten identifizierten Mustern gegenübergestellt. Nachdem die einzelnen Messmodelle bewertet sind, kann das Strukturmodell mit den Daten konfrontiert werden. Inwieweit das Strukturmodell (die operationalisierte Forschungsfrage) als Muster in den empirischen Daten wiederzufinden ist, entscheidet über das Schicksal der Arbeitshypothese und damit über die Antwort auf die in dieser Arbeit gestellte Forschungsfrage.

\subsubsection{1. Überprüfung der Gültigkeit der Messmodelle}

Um dem Leser den Vergleich der durch theoretische Überlegungen hergeleiteten Strukturen mit den in den erhobenen Daten erkannten Mustern möglichst zu erleichtern und Übereinstimmungen und Differenzen schnell erfassbar zu machen, werden die Möglichkeiten der graphischen Darstellung ausgeschöpft. Das Messmodell wird dabei als Grafik abgedruckt, in der die vom Modell unterstellten Zusammenhänge zwischen den Variablen als strichlierte Pfeile dargestellt sind. Das in den erhobenen Daten identifizierte Muster wird in Form von Pfeilen mit durchgezogener Linie abgebildet. Je nachdem ob in der Grafik strichlierte oder durchgezogene Linien die Variablen verbinden kann man die Passung zwischen Messmodell und Datenmuster direkt ablesen. Die Strichstärke der durchgezogenen Pfeile spiegelt die Stärke des erhobenen Zusammenhangs ${ }^{322}$ wider.

322 Die errechneten Korrelationskoeffizienten sind Appendix 2 zu entnehmen. 
5.7.2.1.1. Überprüfung des Messmodells der latenten Variablen „Synergiesystem“

Als erstes ist zu prüfen, inwieweit sich das in Abschnitt 5.4.1. theoretisch hergeleitete Messmodell zur Erfassung der latenten Variablen "Synergiesystem“ als Muster in den empirisch erhobenen Daten wiederfinden lässt. Dazu wird das Messmodell "Synergiesystem“ in Abbildung 44 dargestellt. Die in Abbildung 20 abgebildete Grafik wird hier der Übersichtlichkeit halber um die Aggregationsebene „Kooperation“ reduziert. Die auf der Basis theoretischer Überlegungen unterstellten Beziehungen zwischen den Dimensionen untereinander, sowie den Dimensionen und der latenten Variablen, werden strichliert dargestellt.

Abb. 44: Überprüfung des Messmodells der Variablen „Synergiesystem“

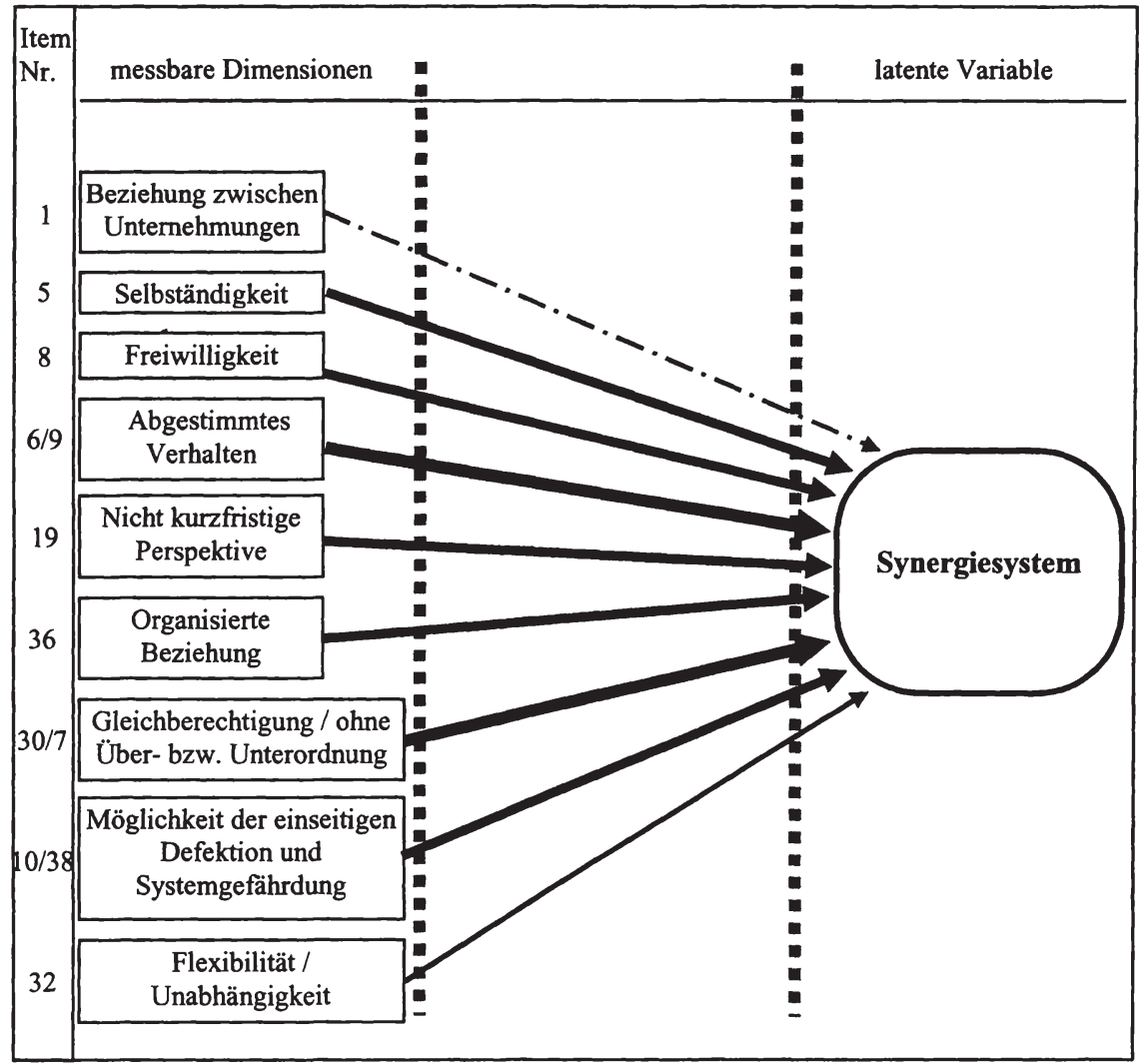


Legt man nun gedanklich das empirisch erhobene Muster über das Messmodell, so überlagern die empirisch vorgefundenen Beziehungen die theoretisch hergeleiteten. Dort, wo eine theoretische Beziehung nicht durch die empirischen Daten bestätigt werden konnte, bleibt die strichlierte Verbindungslinie sichtbar. So wird deutlich, welche theoretischen Überlegungen empirische Bestätigung fanden und welche nicht.

Es zeigt sich, dass die im Messmodell „Synergiesystem“ zusammengefassten Vermutungen über die Zusammenhänge der Dimensionen mit der latenten Variablen "Synergiesystem" in den empirischen Daten weitgehend Bestätigung fanden. Am stärksten stellte sich der Zusammenhang zwischen den Dimensionen „Abgestimmtes Verhalten“ und „Gleichberechtigung“ dar. Etwas weniger stark waren die erhobenen Beziehungen zur latenten Variablen bei den Dimensionen „Selbständigkeit“, „Freiwilligkeit“, „Flexibilität/Unabhängigkeit“, „Nicht kurzfristige Perspektive“, „Organisierte Beziehung“ sowie „Möglichkeit der einseitigen Defektion und Systemgefährdung". Alle erhobenen Korrelationen waren hoch signifikant! ${ }^{323}$

Da nur solche Untersuchungseinheiten auf ihre Synergiesystemeigenschaften untersucht werden konnten, die zum Zeitpunkt der Untersuchung eine Kooperation mit einem anderen Unternehmen unterhielten, beträgt die Korrelation zwischen der Dimension „Beziehung zwischen Unternehmen“ und der latenten Variablen „Synergiesystem“ 1. Da sich dieser Wert durch das Erhebungsdesign ergibt und für die Besprechung daher nicht von Interesse ist, wurde diese Korrelation in die Grafik als strichpunktierter Pfeil eingezeichnet.

Die Prüfung der Güte der Items ergab für das Item „Flexibilität“ ein negatives Resultat. Daher kann über die Beziehung dieser Dimension zur latenten Variablen „Synergiesystem“ keine seriöse Aussage gemacht werden.

Zusammenfassend ist festzustellen, dass alle mit der Itembatterie „Synergiesystem" erfolgreich erhobenen Daten, die theoretisch hergeleiteten Überlegungen über die Zusammenhänge im Rahmen des Messmodells „Synergiesystem“ bestätigen konnten. Das Messmodell erwies sich somit als geeignetes Operationalisierungsinstrument zur Erhebung der Ausprägungen der latenten Variablen "Synergiesystem".

323 In der vorliegenden Arbeit wird von einem "hoch signifikanten" Zusammenhang gesprochen, wenn die Korrelation auf einem Niveau von 0,01 signifikant ist. Korrelationen mit einer Irrtumswahrscheinlichkeit von unter 0,05 werden als "signifikant" bezeichnet. 
5.7.2.1.2. Überprüfung des Messmodells der latenten Variablen „Selbstverpflichtung“"

Wenden wir uns nun dem Messmodell der latenten Variablen „Selbstverpflichtung“ zu. Legt man gedanklich die Folie mit der Darstellung des in den empirischen Daten erkennbaren Musters über das Messmodells, so bietet sich vorerst ein eher ernüchterndes Bild. Nur wenige der strichlierten Pfeile des Messmodells werden durch das empirisch erhobene Muster repräsentierende, durchgezogene Pfeile, überlagert. Der Fit zwischen theoretischer Modellierung und empirischen Regelmäßigkeiten scheint vor allem bezüglich der vorgelagerten Dimensionen gering zu sein.

Abb. 45: Überprüfung des Messmodells der Variablen „Selbstverpflichtung“

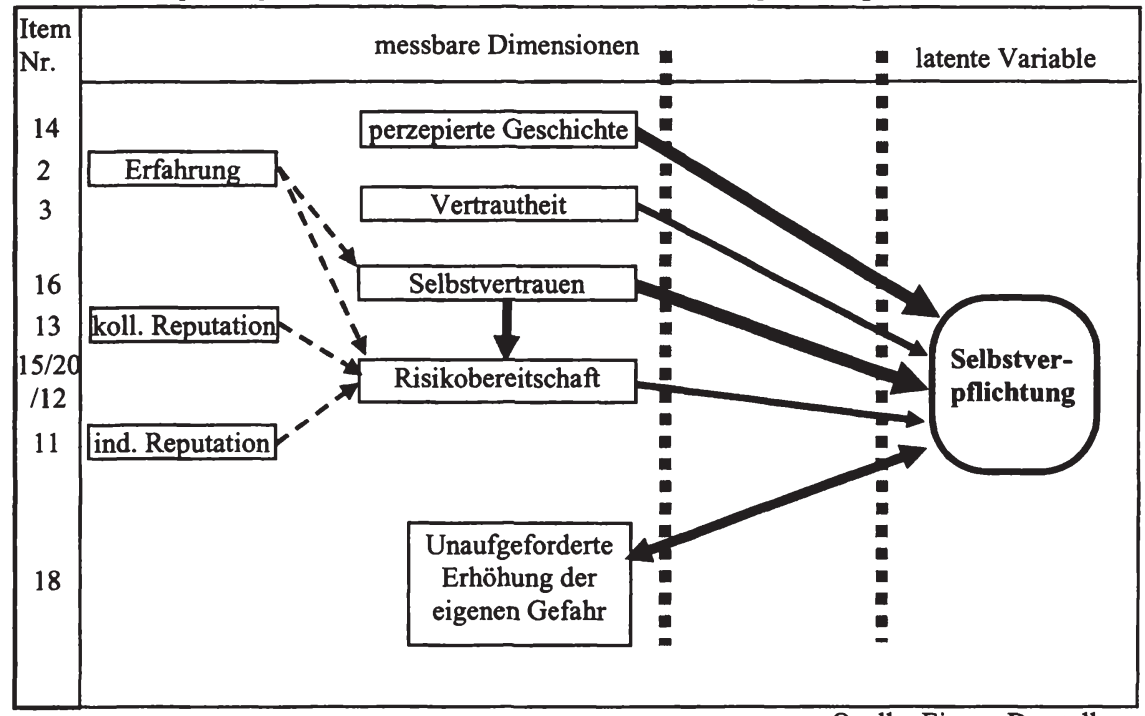

Quelle: Eigene Darstellung

Ist das Messmodell der Variablen „Selbstverpflichtung“ also ungeeignet für die Erhebung der Ausprägungen dieser latenten Variablen? Nein, es ist sehr wohl geeignet, denn es zeigt sich, dass einzig die Sequenzierung (vorgelagerte Dimensionen $\Rightarrow$ Selbstverpflichtung $\Rightarrow$ nachgelagerte Dimension) aufgegeben werden muss, um einen beeindruckenden Fit zwischen dem Modell und den Daten aufzudecken. 
Nun ist jedoch noch zu klären, ob die Aufhebung der Sequenzierung der Elemente des Messmodels „Selbstverpflichtung“ zu rechtfertigen ist. Dazu ist zu prüfen, ob im Zuge der empirischen Erhebung Daten erhoben wurden, die mit den theoretischen Überlegungen zur Sequenzierung der Dimensionen korrespondieren. Es stellt sich heraus, dass keine Information bezüglich der Reihung der Elemente der behaupteten Kausalkette erhoben wurden. Aus den Interkorrelationen der einzelnen Messvariablen lässt sich jedoch darauf schließen, dass die unterstellte Sequenzierung innerhalb des Messmodells „Selbstverpflichtung“ im Lichte der empirischen Daten nicht haltbar ist und daher aufgegeben werden muss. ${ }^{324} / 325$ So stellt sich die Aufgabe der Sequenzierung als nicht nur rechtfertigbar, sondern als notwendig dar. Es ergibt sich somit folgendes, um die Sequenzierung der Elemente reduziertes, Messmodel der latenten Variablen „Selbstverpflichtung“:

Abb. 46: Adaptiertes Messmodell der Variablen „Selbstverpflichtung“

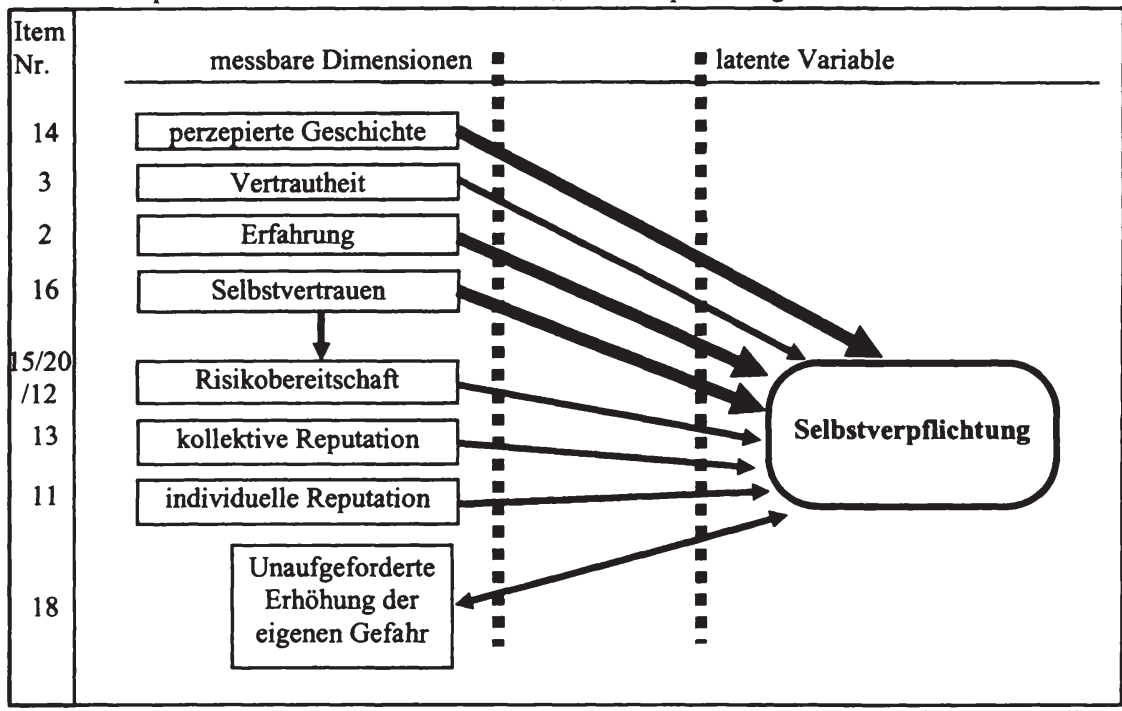

Quelle: Eigene Darstellung

Die Überlagerung des adaptierten Messmodells der Variablen „Selbstverpflichtung" mit dem auf der Folie abgedruckten Muster veranschaulicht den nahezu

324 Würde die behauptete Sequenzierung in der Empirie vorliegen, so müssten die Interkorrelationen zwischen den einzelnen Dimensionen und den ihnen vorgelagerten Dimensionen Werte annehmen, die deutlich über den erhobenen Werten liegen.

325 Zur Notwendigkeit einer Zeitdimension für die Zuweisung von Ursache bzw. Wirkung in Kausalbeziehungen siehe Exkurs III. 
perfekten Fit. Alle theoretisch unterstellten Beziehungen im Messmodell, die in der Abbildung 46 als strichlierte Pfeile dargestellt sind, werden durch grüne Pfeile überlagert. Es ist unmittelbar erkennbar, dass sämtliche unterstellte Beziehungen in den erhobenen Daten empirische Bestätigung fanden. ${ }^{326}$ Der empirisch erhobene Zusammenhang ist zwischen den Dimensionen ,perzepierte Geschichte“, „Erfahrung“, „Selbstvertrauen“ und der latenten Variablen „Selbstverpflichtung“" am stärksten. Da die Auswertung der erhobenen Daten auch für die restlichen Dimensionen einen starken Zusammenhang mit der latenten Variablen ergaben, kann das Messmodell der latenten Variablen „Selbstverpflichtung“ als geeignet gelten, die Ausprägungen dieser latenten Variablen zu erheben.

\subsubsection{3. Überprüfung des Messmodells der vier Perspektiven der latenten Vari- ablen ,Erfolg“}

Als letztes Messmodell ist das Erhebungsschema der latenten Variablen „Erfolg“ auf seine Güte zu prüfen. Inwieweit das Modell mit den in den erhobenen Daten identifizierten Regelmäßigkeiten übereinstimmt, wird ebenso wie bei den beiden anderen Messmodellen anhand von graphischen Darstellungen besprochen. Überlagert man das in Abbildung 47 dargestellte Modell mit den aus den empirischen Daten extrahierten Beziehungen so zeigt sich das Ausmaß der Übereinstimmung.

Der Betrachter erkennt, dass sämtliche strichlierte Pfeile durch einen grünen Pfeil abgedeckt werden. Jede im Bereich dieses Messmodells unterstellte Beziehung wurde von den in den empirischen Daten identifizierten Regelmäßigkeiten bestätigt. D.h. das Modell konnte als Muster in den Daten wiedergefunden werden. Damit stellt sich auch das dritte Messmodell als geeignet dar, die Ausprägungen der latenten Variablen „Erfolg“ aus den vier hier berücksichtigten Perspektiven ${ }^{327}$ zu erheben.

326 Obwohl sich Item Nummer 15 im Zuge des Konsistenztests als nicht geeignet herausstellte, die Dimension "Risikobereitschaft" zu erfassen, konnte diese Dimension mit den Items Nummer 12 und Nummer 20 dennoch erfasst werden.

327 Die vier hier berücksichtigten Perspektiven der latenten Variablen "Erfolg" sind: 1. Erfolg aus der Beziehungsperspektive, 2. Erfolg aus der Binnenperspektive, 3. Erfolg aus der Außenperspektive, 4. Erfolg aus der Finanzperspektive. 
Abb. 47: Überprüfung des Messmodells der Variablen „Erfolg“

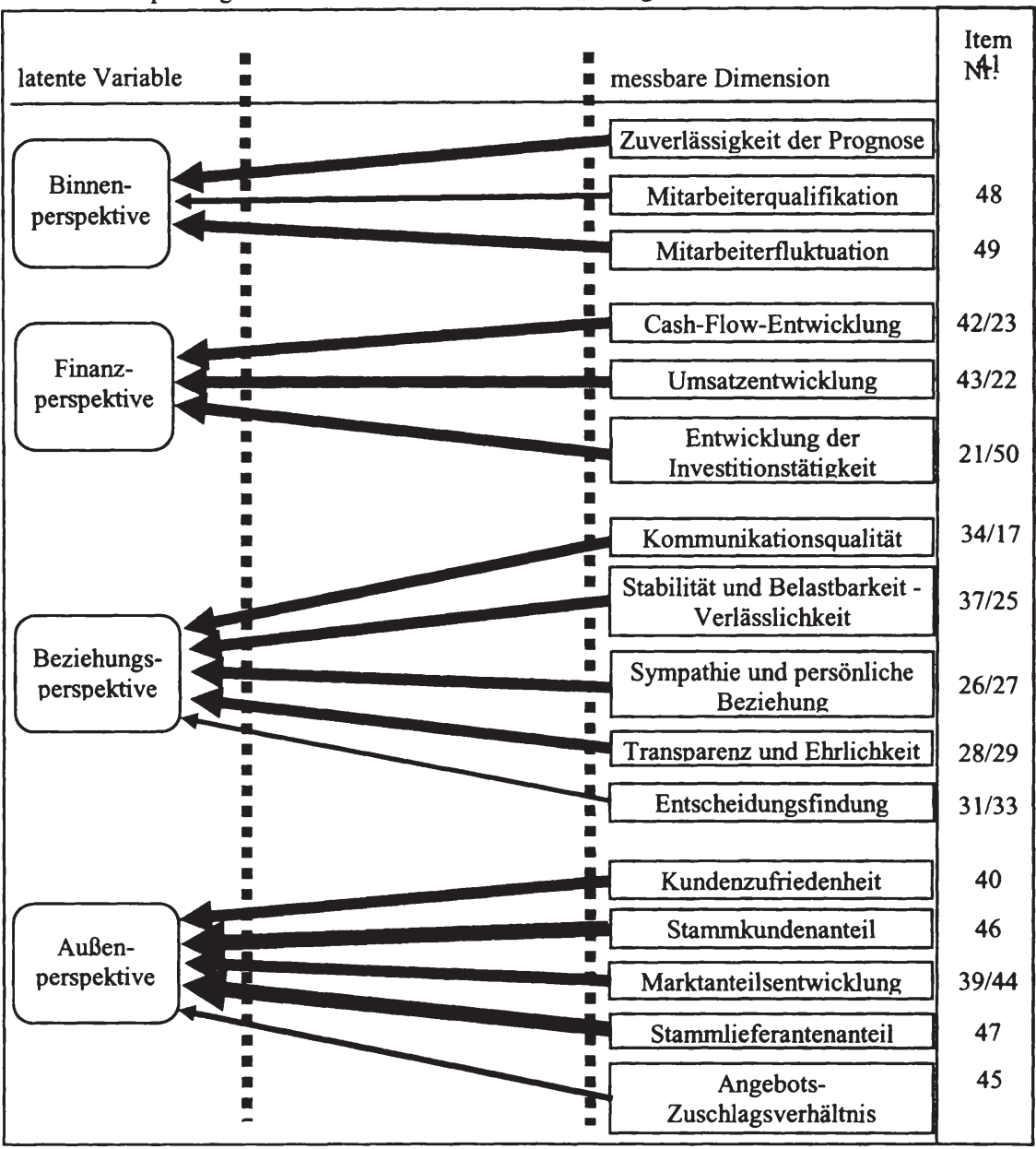

Quelle: Eigene Darstellung

Nachdem die Forschungsfrage im Zuge einer sukzessiven Operationalisierung bis auf die Items im Fragebogen heruntergebrochen wurde, konnten die einzelnen Stufen dieses Messbarmachungsprozesses schrittweise an den empirischen Daten getestet werden. Nach der Überprüfung der Güte der Einzelitems und der verwendeten Messmodelle ist die inhaltliche Entwicklung des Textes wieder auf der Aggregationsebene des Strukturmodells angelangt. 
5.7.2.1.4. Überprüfung der Struktur der verwendeten Balanced Scorecard

Im Zuge der Operationalisierung der latenten Variablen Erfolg wurde eine Balanced Scorecard entworfen (Abschnitt 5.1.3.), die den Erfolg der befragten Unternehmen aus den vier Perspektiven (Beziehungsperspektive, Binnenperspektive, Außenperspektive und Finanzperspektive) beleuchtete. Durch den Entwurf der Struktur dieser Balanced Sorecard wurde implizit eine Aussage über die Wirkungszusammenhänge zwischen den einzelnen Perspektiven getroffen. Es wurde unterstellt, dass sich Entwicklungen der Dimensionen, die von der Beziehungsperspektive erfasst werden, sowohl auf den Erfolg des Unternehmens nach innen (Binnenperspektive) als auch auf den Erfolg des Unternehmens nach außen (Außenperspektive) auswirken und, dass solche Entwicklungen in einem zweiten Schritt den aus der Finanzperspektive erfassten Erfolg des Unternehmens beeinflussen.

Die unterstellten Wirkungsbeziehungen sind in Abbildung 48 als strichlierte Pfeile dargestellt. Um zu überprüfen, inwieweit sich diese unterstellten Zusammenhänge als Muster in den erhobenen Daten wiederfinden lassen, wurde aus den Daten für jedes Variablenpaar ein Korrelationskoeffizient errechnet und in einen Pfeil übersetzt auf einer gedanklich höher liegenden Ebene abgedruckt. Der Abgleich der beiden Strukturen zeigt das Ausmaß der Übereinstimmung der unterstellten Wirkungszusammenhänge mit dem empirisch erhobenen Muster.

Abb. 48: Überprüfung der Struktur der Balanced Scorecard

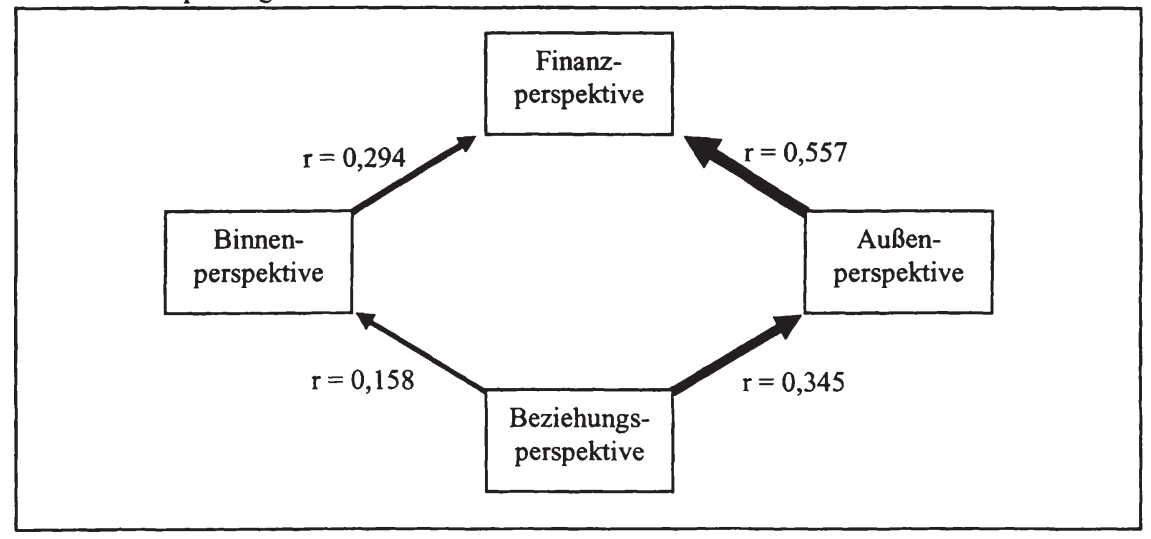

Quelle: Eigene Darstellung 
Es zeigt sich, dass die unterstellten Wirkungszusammenhänge nur teilweise Bestätigung in den empirischen Daten finden. So ist der Korrelationskoeffizient, der für die Variablen Erfolg aus der Beziehungsperspektive und Erfolg aus der Binnenperspektive errechnet wurde, äußerst bescheiden $(r=0,158)$. Deutlich stärker ist der aus den Daten errechnete Zusammenhang zwischen den Variablen Erfolg aus der Beziehungsperspektive und Erfolg aus der Außenperspektive $(r=0,345)$. Analog verhält es sich mit den beiden Korrelationskoeffizienten, die sich aus den Daten für die Variablen Erfolg aus der Binnenperspektive und Erfolg aus der Finanzperspektive einerseits und Erfolg aus der Außenperspektive und Erfolg aus der Finanzperspektive andererseits ergeben. Während für das erste Variablenpaar nur ein eher schwacher Zusammenhang $(r=0,294)$ festgestellt wurde, ist dieser für das zweite Variablenpaar deutlich stärker ausgeprägt $(\mathrm{r}=0,557)$.

Die Ergebnisse der empirischen Überprüfung der, im Rahmen der verwendeten Balanced Scorecard, implizit unterstellten Wirkungszusammenhänge, deuten auf die relativ größere Bedeutung der von der Variablen Erfolg aus der Außenperspektive erfassten Dimensionen für den finanziellen Erfolg des Unternehmens hin. Eine hohe Kundenzufriedenheit, ein hoher Stammkunden- und Stammlieferantenanteil, ein wachsender Marktanteil und eine gute Entwicklung der Angebots-Zuschlagesentwicklung (Erfolg aus der Außenperspektive) scheinen für den finanziellen Erfolg deutlich wichtiger zu sein, als zuverlässige Prognosen, eine sinkende Mitarbeiterfluktuation sowie eine steigende Mitarbeiterqualifikation (Erfolg aus der Binnenperspektive). Stark simplifizierend könnte man zusammenfassen: Solange das Unternehmen seine Produkte am Markt absetzt und damit die Kunden zufrieden stellt, hat es auch finanziellen Erfolg. Interne Aspekte sind dafür nur von untergeordneter Bedeutung.

\subsubsection{2. Überprüfung der Gültigkeit des Strukturmodells}

Die Konfrontation des Strukturmodells mit den empirischen Daten erfolgt analog zu den Überprüfungen der Messmodelle. Die theoretisch unterstellten Zusammenhänge sind in Abbildung 49 durch strichlierte Pfeile dargestellt. Die in den empirischen Daten identifizierten korrespondierenden Regelmäßigkeiten sind auf einer gedanklich darüber liegenden Ebene abgedruckt. Anders als bei der Überprüfung der Messmodelle, werden hier nicht unterstellte Zusammenhänge zwischen Dimensionen betrachtet, sondern es werden die auf der Basis theoretischer Überlegungen aufgestellten Hypothesen über die Beziehungen zwischen den latenten Variablen mit der Empirie konfrontiert. Das Vorgehen ist das gleiche, die betrachtete Aggregationsebene unterscheidet sich jedoch! 
Abb. 49: Überprüfung des Strukturmodells

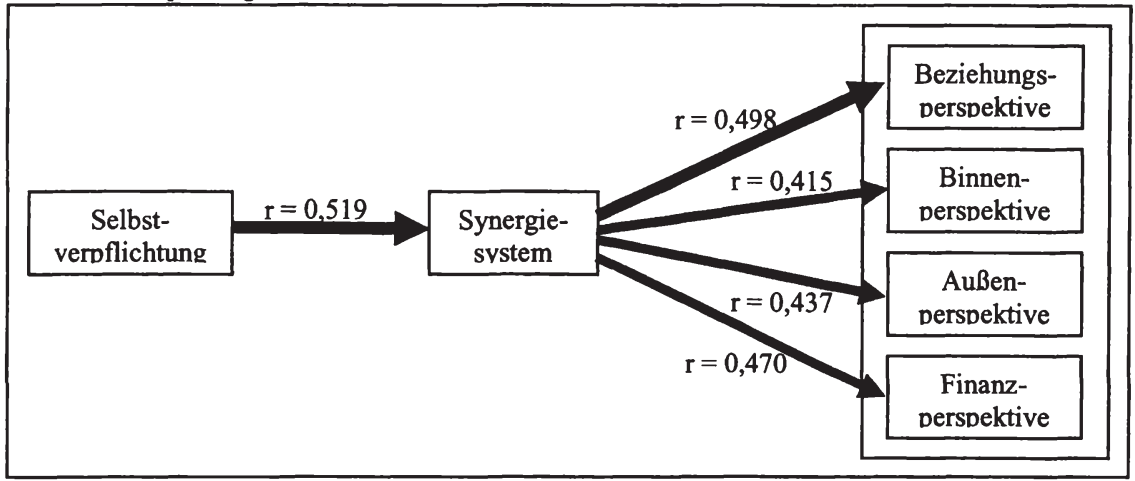

Quelle: Eigene Darstellung

Legt man nun die Folie über Abbildung 49, so zeigt sich, dass sämtliche auf der Basis der theoretischen Argumentation formulierten Hypothesen über die Beziehungen der latenten Variablen untereinander, in den empirischen Daten ihre Entsprechung finden. Die Analyse der erhobenen Daten macht Regelmäßigkeiten sichtbar, die den theoretisch unterstellten Beziehungen entsprechen.

Das Strukturmodell stellt die letzte Operationalisierungsebene vor der Arbeitshypothese dar. Das Strukturmodell konnte anhand der empirischen Daten bestätigt werden. Es gilt nun über das Schicksal der Arbeitshypothese zu entscheiden. Die Arbeitshypothese war der Ausgangspunkt der empirischen Erhebung. Thre Konfrontation mit den erhobenen Daten stellt den logischen Schlusspunkt der textlichen Entwicklung dar.

\subsection{3. ÜBERPRÜFUNG DER ARBEITSHYPOTHESE - DAS ERGEBNIS DER EMPIRISCHEN UNTERSUCHUNG}

Die empirische Untersuchung setzte sich zum Ziel, die Gültigkeit der in Abschnitt 3 entwickelten Argumentation an der Empirie zu testen. Dazu wurde das Initialmoment der theoretisch argumentierten Kausalkette mit deren Endpunkt in eine quantifizierte Beziehung gesetzt. Dahinter steht die Idee, dass eine Überprüfung der behaupteten Relation zwischen auslösendem Moment und Ergebnis der Wirkungskette gleichzeitig den Argumentationsweg überprüfbar macht. Stimmt der argumentativ prognostizierte Endpunkt nicht mit dem empirisch erhobenen Ergebnis der Kausalkette überein, so ist die Argumentation als empirisch irrelevant zu werten. Abbildung 50 stellt diesen Zusammenhang schematisch dar. 
Abb. 50: Die Arbeitshypothese

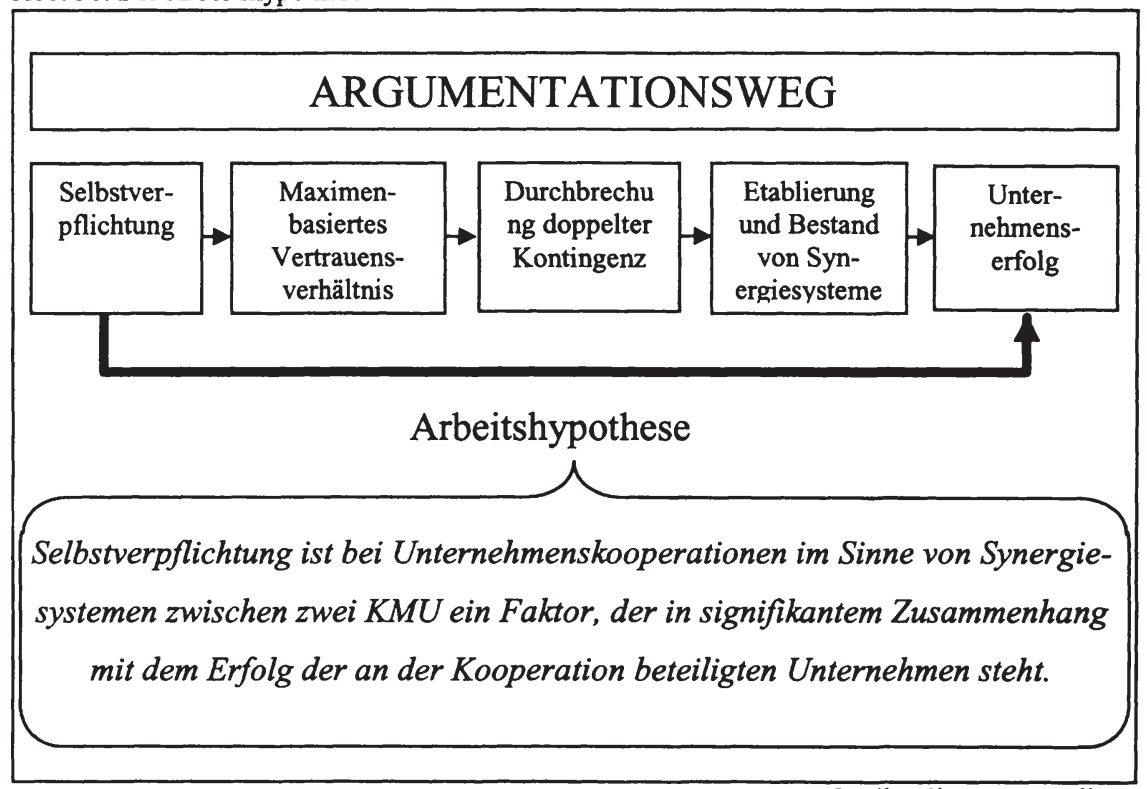

Quelle: Eigene Darstellung

Bevor nach diesem Schema vorgegangen werden kann, ist jedoch noch ein theoretisches Problem zu bewältigen: Bei diesem Vorgehen besteht immer die Möglichkeit, dass zwar die Arbeitshypothese durch die empirischen Daten Bestätigung findet, der Argumentationsweg, auf dessen Basis die Arbeitshypothese formuliert wurde, dennoch nicht zutrifft. Prüft man nur Startpunkt und Endpunkt einer Kausalkette (klassischer Hypothesentest) so ist es durchaus plausibel anzunehmen, dass zwischen den beiden Elementen, deren Beziehung als Arbeitshypothese formuliert wurde, eine andere Kausalkette als die behauptete existiert. Der Rückschluss vom Test der Arbeitshypothese auf die Gültigkeit des Argumentationsweges ist dann logisch nicht zu rechtfertigen. Um diese Problem zu entschärfen, wird die Arbeitshypothese in der vorliegenden Untersuchung in zwei Teilhypothesen zerlegt: 
Teilhypothese I: Die Selbstverpflichtung der Kooperateure ist eine Voraussetzung für die Etablierung und den Bestand einer Unternehmenskooperation im Sinne von Synergiesystemen zwischen zwei $K M U$.

Teilhypothese II: Die Etablierung und der Bestand einer Unternehmenskooperation im Sinne von Synergiesystemen zwischen zwei KMU hat einen signifikant positiven Einfluss auf den Erfolg der an der Kooperation beteiligten Unternehmen.

So kann im Rahmen der empirischen Untersuchung auch ein wichtiger Zwischenschritt des Argumentationsweges überprüft werden. Damit wurde die Sicherheit des Rückschlusses von der Arbeitshypothese auf den Argumentationsweg soweit erhöht, dass von einer Überprüfung der Gültigkeit des Argumentationsweges gesprochen werden kann.

Abb. 51: Hypothesentest als Überprüfung der Gültigkeit des Argumentationsweges

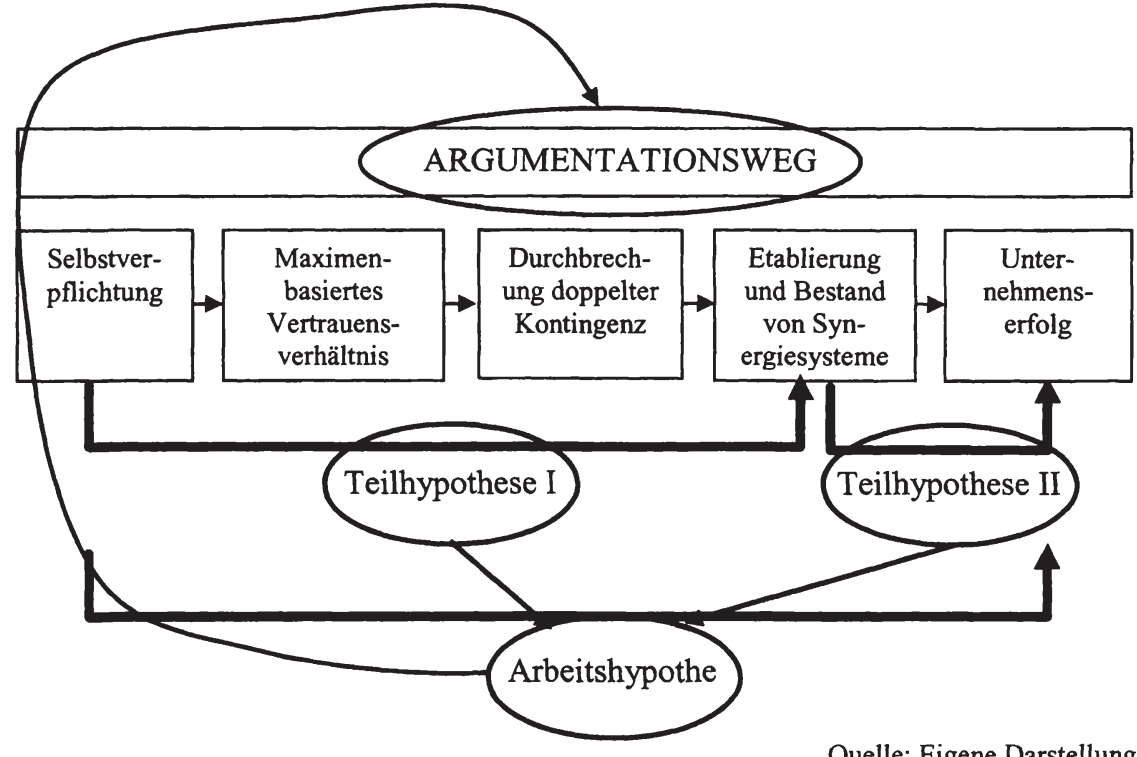

Quelle: Eigene Darstellung

Folgt man dem in Abbildung 50 dargestellten Schema, so ist als erster Schritt die Teilhypothese I mit den empirischen Daten zu konfrontieren. Es ist zu prüfen, ob sich in den Daten ein Muster erkennen lässt, dass der behaupteten Beziehung zwi- 
schen den latenten Variablen „Selbstverpflichtung“ und „Synergiesystem“ entspricht. Die Überprüfung auf regelmäßige Zusammenhänge im Datenmaterial wird mittels Berechnung von Korrelationskoeffizienten durchgeführt. Für die zwei hier betrachteten Variablen ergibt sich eine hoch signifikante Korrelation nach Pearson von 0,519 (vgl. Abb. 49). In den erhobenen Daten besteht somit ein relativ starker Zusammenhang zwischen der Stärke der Selbstverpflichtung eines Kooperateurs und der Stärke der Synergiesystemeigenschaften seiner wichtigsten Kooperation. Die in Teilhypothese I behauptete Relation, wonach die Selbstverpflichtung des Kooperateurs eine Voraussetzung für die Etablierung und den Bestand eines Synergiesystems ist, kann somit als durch die empirische Erhebung bestätigt gelten.

Als zweiter Schritt ist die Gültigkeit der Teilhypothese II anhand der erhobenen Daten zu überprüfen. Da die latente Variable „Erfolg“ im Rahmen der empirischen Untersuchung aus vier verschiedenen Perspektiven erhoben wurde, muss die Gültigkeit der Teilhypothese II für jede Perspektive der latenten Variablen „Erfolg“ gesondert durchgeführt werden. Die Berechung der Korrelationskoeffizienten nach Pearson ergibt folgende Werte:

Abb. 52: Test der Teilhypothese II - Korrelationskoeffizienten

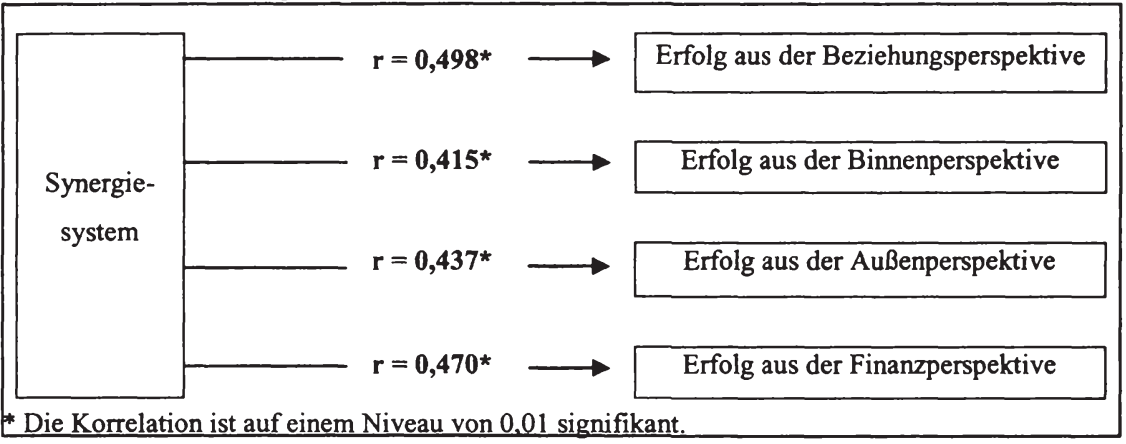

Quelle: Eigene Darstellung

Die Variable „Synergiesystem“ ist mit allen vier Perspektiven der Variablen „Erfolg“ ausreichend stark korreliert, um den in Teilhypothese II formulierten Zusammenhang als empirisch bestätigt bezeichnen zu können. 
Da der empirische Gehalt der zwei Teilhypothesen den Aussagegehalt der Arbeitshypothese erschöpft ${ }^{328}$, ist auch die Arbeitshypothese durch die Bestätigung der zwei Teilhypothesen bestätigt.

Die empirische Untersuchung kommt zu dem Ergebnis, dass Selbstverpflichtung bei Unternehmenskooperationen im Sinne von Synergiesystemen zwischen zwei KMU ein Faktor ist, der in signifikantem (positivem) Zusammenhang mit dem Erfolg der an der Kooperation beteiligten Unternehmen steht.

Außerdem kann auf der Basis der oben geführten Argumentation von der Bestätigung der Arbeitshypothese auf die Gültigkeit des zugrundeliegenden Argumentationsweges geschlossen werden. ${ }^{329}$ Es kann somit festgestellt werden:

Die empirische Untersuchung kann den Argumentationsweg bestätigen, wonach die Selbstverpflichtung der Akteure eine notwendige Voraussetzung für die Etablierung eines maximenbasierten Vertrauensverhältnisses ist und der Bestand eines maximenbasierten Vertrauensverhältnisses eine notwendige Voraussetzung für die Durchbrechung der doppelten Kontingenz ist. Das ist wiederum eine notwendige Voraussetzung für die Etablierung und den Bestand von Transaktionsbeziehungen, die auf langfristige Vorteile abzielen und hochgradig unsicher und komplex sind (Synergiesysteme). Es konnte gezeigt werden, dass sich die Etablierung und der Bestand eines solchen Synergie-systems positiv auf den Erfolg der beteiligten kleinen und mittleren Unternehmen auswirkt.

328 Die Teilhypothesen wurde aus der Arbeitshypothese deduziert, beinhalten also keine zusätzlichen Inhalte.

329 Es handelt sich dabei um einen induktiven Schluss auf der Basis von Wahrscheinlichkeiten. 


\section{Zusammenfassende Thesen - Was wurde erreicht?}

These 1:

Erst das Verständnis von Austauschbeziehungen als Interaktionen unvollständig informierter, begrenzt rationaler, sowie begrenzt opportunistischer Wirtschaftssubjekte, macht eine Analyse von Unsicherheit, Vertrauen und Komplexität möglich und eröffnet so einen gangbaren Weg, die Durchbrechung der doppelten Kontingenz (Lösung des Gefangenendilemmas) argumentierbar zu machen.

\section{These 2:}

Indem vermeintliche Sachzwänge als Denkzwänge entlarvt wurden und die $\mathrm{Zu}$ mutbarkeit kooperativen Verhaltens für die Wirtschaftssubjekte aufgezeigt wurde, konnte intrinsisch motiviertes (maximenbasiertes) Vertrauen als gebotene Basis für die Selbstverpflichtung der Wirtschaftssubjekte von seiner ihm, im traditionellen wirtschaftswissenschaftlichen Diskurs zugewiesenen Rolle, als realitätsfremde Zukunftsutopie heillos optimistischer Idealisten befreit werden.

\section{These 3:}

Es wurde geklärt, warum sich der Wirtschaftsakteur auf der Basis maximenbasierten Vertrauens kooperativ verhalten kann und soll.

These 4:

Es ist gelungen die Notwendigkeit der Selbstverpflichtung der Akteure für die Durchbrechung der doppelten Kontingenz und damit für die Etablierung und den Bestand hochgradig komplexer Transaktionsbeziehungen, die auf eine langfristige Zielerreichung angelegt sind (Synergiesysteme), theoretisch stringent $\mathrm{zu}$ argumentieren. Damit wurde Selbstverpflichtung als einzig möglicher Koordinationsmechanismus für Synergiesysteme identifiziert.

These 5:

Das Verständnis der Phänomene Selbstverpflichtung und Synergiesystem wurde vorangetrieben, indem einerseits eine Reihe wichtiger Faktoren identifiziert wurde, die den Grad der Selbstverpflichtung der Wirtschaftsakteure mitbestimmen und andererseits entscheidende Entstehungsbedingungen und Auswirkungen 
von Synergiesystemen theoretisch erfasst wurden. Die theoretische Aufarbeitung der Phänomene Selbstverpflichtung und Synergiesystem, sowie die Entwicklung einer maßgeschneiderten Balanced Score Card für die empirische Erfassung des Unternehmenserfolgs, ermöglichten die großzahlige empirische Überprüfung der Auswirkungen der Selbstverpflichtung auf den Erfolg von an Synergiesystemen beteiligten Unternehmen.

These 6:

Die entscheidende Rolle der Selbstverpflichtung bei der Etablierung und dem Bestand von langfristigen, hochgradig komplexen Transaktionsbeziehungen (Synergiesystemen) konnte auch im Rahmen der empirischen Untersuchung bestätigt werden.

These 7:

Ebenso konnten die positiven Auswirkungen der Etablierung eines Synergiesystems auf den Erfolg der beteiligten Unternehmen empirisch bestätigt werden.

These 8:

In vorliegender Arbeit ist es gelungen, die Rolle der Selbstverpflichtung der Wirtschaftsakteure als Erfolgsfaktor bei Synergiesystemen unter KMU sowohl theoretisch, argumentativ herzuleiten, als auch empirisch zu bestätigen. 
Appendix 1: Itemnummer, Label (SPSS 10) und Itemformulierung 
Item Nr. im|Kurzbe- $\quad$ Formulierung des Items - Im Fragebogen gestellte Frage

Fragebogen zeich-

nung der

Mess-

variablen

\begin{tabular}{l|l|l}
\hline Filter & kooperat & Ich unterhalte zu Zeit eine Kooperation (Enge Zusammenarbeit) mit einem anderen Unternehmen. \\
\hline 1 & jahre & Die Kooperation besteht seit ... Jahren. \\
\hline 2 & erstkoop & Diese Kooperation ist meine erste Kooperation. \\
\hline 3 & früherko & Ich habe mit meinem jeztigen Kooperationspartner schon früher einmal kooperiert. \\
\hline 5 & intensiv & Die Kooperation ist seit ihrem Beginn intensiver geworden. \\
\hline 6 & rechtsel & Ich bin in dieser Kooperation rechtlich selbständig. \\
\hline 7 & abstverh & Ich stimme mein Verhalten mit meinem Kooperationspartner ab. \\
\hline 8 & gleiinte & Ich denke, mein Kooperationspartner und ich haben gleich starkes Interesse an der Kooperation. \\
\hline 10 & jedbeend & Ich kann die Kooperation jederzeit einseitig beenden. \\
\hline 11 & gemvorge & Mein Kooperationspartner und ich gehen in bestimmten Bereichen gemeinsam vor. \\
\hline 12 & ichego & Ich könnte durch egoistisches Verhalten meinem Kooperationspartner Schaden zufügen. \\
\hline 13 & gutkoop & Ich habe vor der Kooperation Gutes über meinen jezigen Kooperationspartner gehört. \\
\hline 14 & einflerf & Die Kooperation hat einen großen Einfluß auf den Erfolg meines Unternehmens. \\
\hline 15 & brancgut & Ich habe vor der Kooperation Gutes über die Branche gehört, in der mein jeziger Kooperationspartner tätig ist. \\
\hline 16 & infopart & Ich habe mich vor der Kooperation über meinen Kooperationspartner informiert. \\
\hline 17 & kooprisk & Jede Kooperation stellt für die beteiligten Unternehmen ein Risiko dar. \\
\hline
\end{tabular}




\begin{tabular}{|c|c|c|}
\hline 18 & immrisk & $\begin{array}{l}\text { Damit eine Kooperation gelingt, muss man seinem Kooperationspartner einen Vertrauensvorschuss geben, auch wenn man } \\
\text { damit ein Risiko eingeht. }\end{array}$ \\
\hline 19 & schnerfo & Ich möchte durch die Kooperation möglichst schnell sichtbaren Erfolg erzielen. \\
\hline 20 & riskok & Ich bin bereit ein Risiko einzugehen. \\
\hline 21 & mehrinv & Seit die Kooperation besteht, habe ich verstärkt in mein Unternehmen investiert. \\
\hline 22 & umsplus & Ich konnte seit Beginn der Kooperation meinen Umsatz deutlich steigern. \\
\hline 23 & cfplus & Ich konnte seit Beginn der Kooperation meinen Cash-Flow deutlich steigern. \\
\hline 24 & kopmitar & Seit Bestand der Kooperation haben weniger Mitarbeiter mein Unternehmen verlassen als zuvor. \\
\hline 25 & diskerg & Diskussionen mit meinem Kooperationspartner führen immer zu einem Ergebnis. \\
\hline 26 & privkont & Ich habe auch privat Kontakt zu meinem Kooperationspartner. \\
\hline 27 & koplmens & Mein Kooperationspartner ist ein komplizierter Mensch. \\
\hline 28 & ablkenne & Ich kenne die Abläufe im Unternehmen meines Kooperationspartners. \\
\hline 29 & nachfrag & $\begin{array}{l}\text { Auf Nachfrage bekomme ich immer die relevanten Informationen über die Abläufe im Unternehmen meines } \\
\text { Kooperationspartners. }\end{array}$ \\
\hline 30 & entschgl & Bei Entscheidungen, die die Kooperation betreffen, hat die Stimme jedes Kooperationspartners gleich viel Gewicht. \\
\hline 31 & größeinf & Bei Entscheidungen habe ich einen größeren Einfluss auf das Ergebnis als mein Kooperationspartner. \\
\hline 32 & außeinfl & $\begin{array}{l}\text { Entscheidungen, die die Kooperation betreffen, werden ausschließlich von meinem Kooperationspartner und mir getroffen. } \\
\text { Es gibt keinen Einfluß von außen. }\end{array}$ \\
\hline 33 & taktik & Mein Kooperationspartner ist ein großer Taktiker. \\
\hline 34 & erreichb & Mein Kooperationspartner ist für mich stets erreichbar. \\
\hline 35 & mitqualk & Seit Beginn der Kooperation hat sich die Qualifikation meiner Mitarbeiter erhöht. \\
\hline 36 & sprekoop & Mein Kooperationspartner und ich sprechen über die Kooperation. \\
\hline 37 & sprprobl & Ich kann mit meinem Kooperationspartner offen über Probleme sprechen. \\
\hline 38 & egoschk & Mein Kooperationspartner könnte mir durch egoistisches Verhalten Schaden zufügen. \\
\hline
\end{tabular}




\begin{tabular}{l|l|l}
39 & mantlplu & Ich habe seit Bestand der Kooperation meinen Marktanteil vergrößert. \\
\hline 40 & kundzufr & Meine Kunden sind stets mit den Leistungen meines Unternehmens zufrieden. \\
\hline 41 & prognose & Die Prognosen meines Unternehmens über den Geschäftsverlauf sind verlässlich. \\
\hline 43 & cfplu3 & Ich konnte in den letzten drei Jahren meinen Cash-Flow deutlich steigern. \\
\hline 44 & umsplu3 & Ich konnte in den letzten drei Jahren meinen Umsatz deutlich steigern. \\
\hline 45 & markplu3 & Ich konnte in den letzten drei Jahren meinen Marktanteil deutlich vergößern. \\
\hline 46 & angebzus & In den letzten drei Jahren ist es schwieriger geworden bei Angeboten den Zuschlag zu bekommen. \\
\hline 48 & stamkund & Die meisten meiner Kunden sind Stammkunden. \\
\hline 49 & stamlief & Die meisten meiner Lieferanten sind Stammlieferanten. \\
\hline 50 & mitqua3 & Die Qualifikation meiner Mitarbeiter konnte in den letzten drei Jahren gesteigert werden. \\
\hline
\end{tabular}


Appendix 2: Datenmatrix 


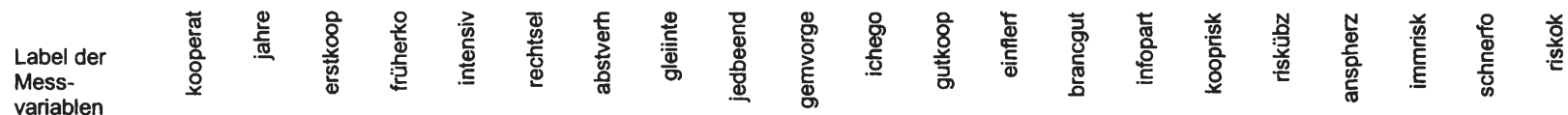

\begin{tabular}{|c|c|c|c|c|c|c|c|c|c|c|c|c|c|c|c|c|c|c|c|c|c|c|}
\hline Fall & 1 & 1 & 5 & 1 & 1 & 3 & 4 & 3 & 3 & 1 & 3 & 3 & 3 & 4, & & 4 & 3 & 3 & 2 & 3 & 2 & 2 \\
\hline Fall & 2 & 1 & 3 & 4 & 1 & 4 & 4 & 4 & 4 & 3 & 4 & 4 & 3 & 3. & & 3 & 4 & 4 & 4 & 4 & 2 & 4 \\
\hline Fall & 3 & 1 & 2 & 4 & 4 & 4 & 4 & 4 & 3 & 3 & 4 & 3 & 3 & 3 & 1 & 2 & 3 & 3 & 3 & 3 & 2 & 3 \\
\hline Fall & 4 & 1 & 4 & 1 & 4 & 4 & 4 & 4 & 4 & 4 & 4 & 3 & 2 & 4 & 3 & 3 & 2 & 3 & 4 & 3 & 2 & 2 \\
\hline Fall & 5 & 1 & 6 & 4 & 4 & 4 & 4 & 4 & 4 & 4 & 2 & 3 & 4 & 3 & 3 & 3 & 3 & 3 & 4 & 3 & 1 & 3 \\
\hline Fall & 6 & 1 & 10 & 1 & 1 & 4 & 4 & 2 & 3 & 3 & 2 & 3 & 2 & 3 & 2 & 2 & 3 & 3 & 4 & 3 & 1 & 3 \\
\hline Fall & 7 & 1, & & 1 & 1 & 2 & 2 & 3 & 3 & 3 & 2 & 4 & 3 & 3 & 3 & 3 & 4 & 3 & 3 & 3 & 2 & 3 \\
\hline Fall & 8 & 1 & 2 & 1 & 4 & 3 & 3 & 3 & 3 & 4 & 4 & 2 & 3 & 4 & 3 & 3 & 2 & 3 & 4 & 3 & 2 & 2 \\
\hline Fall & 9 & 1 & 6 & 1 & 1 & 2 & 3 & 4 & 3 & 3 & 3 & 4 & 3 & 2 & 3 & 2 & 3 & 2 & 3 & 2 & 2 & 2 \\
\hline Fall & 10 & 1 & 8 & 4 & 4 & 4 & 4 & 3 & 3 & 2 & 3 & 3 & 3 & 3 & 3 & 4 & 3 & 3 & 3 & 3 & 2 & 3 \\
\hline Fall & 11 & 1 & 3 & 4 & 1 & 3 & 3 & 3 & 4 & 3 & 3 & 2 & 3 & 2 & 3 & 3 & 3 & 3 & 3 & 3 & 2 & 3 \\
\hline Fall & 12 & 1 & 18 & 4 & 1 & 2 & 3 & 3 & 3 & 3 & 3 & 3 & 3 & 2 & 3 & 3 & 3 & 3 & 3 & 3 & 2 & 3 \\
\hline Fall & 13 & 1 & 3 & 4 & 1 & 3 & 4 & 3 & 3 & 4 & 3 & 4 & 2 & 3 & 3 & 3 & 2 & 3 & 3 & 4 & 1 & 3 \\
\hline Fall & 14 & 1 & 5 & 4 & 4 & 3 & 4 & 3 & 3 & 4 & 3 & 3 & 3 & 2 & 1 & 4 & 2 & 3 & 2 & 4 & 2 & 3 \\
\hline Fall & 15 & 1 & 10 & 4 & 1 & 3 & 3 & 4 & 4 & 2 & 3 & 3 & 3 & 3 & 2 & 3 & 3 & 3 & 3 & 3 & 2 & $\underline{3}$ \\
\hline Fall & 16 & 1 & 8 & 1 & 1 & 1 & 4 & 3 & 3 & 2 & 3 & 3 & 4 & 4 & 3 & 4 & 2 & 3 & 3 & 3 & 2 & 2 \\
\hline Fall & 17 & 1 & 5 & 1 & 1 & 4 & 4 & 4 & 4 & 4 & 4 & 2 & 1 & 4 & 3 & 4 & 4 & 3 & 4 & 4 & 2 & 3 \\
\hline Fall & 18 & 1 & 5 & 1 & 1 & 3 & 4 & 2 & 3 & 4 & 2 & 3 & 2 & 2 & 1 & 2 & 3 & 3 & 3 & 4 & 3 & 3 \\
\hline Fall & 19 & 1 & 2 & 4 & 1 & 3 & 4 & 3 & 3 & 4 & 3 & 3 & 2 & 3 & 2 & 3 & 4 & 3 & 4 & 3 & 2 & $\underline{3}$ \\
\hline Fall & 20 & 1 & 16 & 4 & 4 & 3 & 3 & 2 & 3 & 4 & 2 & 3 & 3 & 3 & 2 & 2 & 3 & 3 & 3 & 3 & 2 & 3 \\
\hline Fall & 21 & 1 & 6 & 4 & 4 & 3 & 3 & 3 & 3 & 3 & 3 & 3 & 3 & 2 & 3 & 3 & 3 & 3 & 3 & 3 & 3 & 3 \\
\hline Fall & 22 & 1 & 3 & 4 & 1 & 3 & 4 & 4 & 4 & 2 & 4 & 4 & 4 & 4 & 3 & 4 & 4 & 4 & 4 & 3 & 2 & 3 \\
\hline Fall & 23 & 1 & 10 & 1 & 1 & 3 & 4 & 3 & 3 & 4 & 3 & 3 & 3 & 3 & 3 & 3 & 2 & 3 & 3 & 3 & 2 & 2 \\
\hline Fall & 24 & 1 & 11 & 1 & 1 & 3 & 4 & 3 & 3 & 3 & 4 & 3 & 1 & 2 & 3 & 3 & 4 & 4 & 3 & 4 & 3 & 3 \\
\hline Fall & 25 & 1 & 6 & 4 & 1 & 3 & 4 & 3 & 3 & 4 & 3 & 3 & 2 & 3 & 3 & 3 & 4 & 3 & 3 & 3 & 3 & 3 \\
\hline Fall & 26 & 1 & 8 & 1 & 1 & 3 & 4 & 4 & 4 & 4 & 4 & 4 & 3 & 4 & 3 & 3 & 4 & 4 & 3 & 3 & 3 & 4 \\
\hline Fall & 27 & 1 & 6 & 1 & 4 & 3 & 3 & 4 & 3 & 4 & 4 & 3 & 4 & 4 & 3 & 4 & 3 & 3 & 4 & 4 & 3 & 3 \\
\hline
\end{tabular}




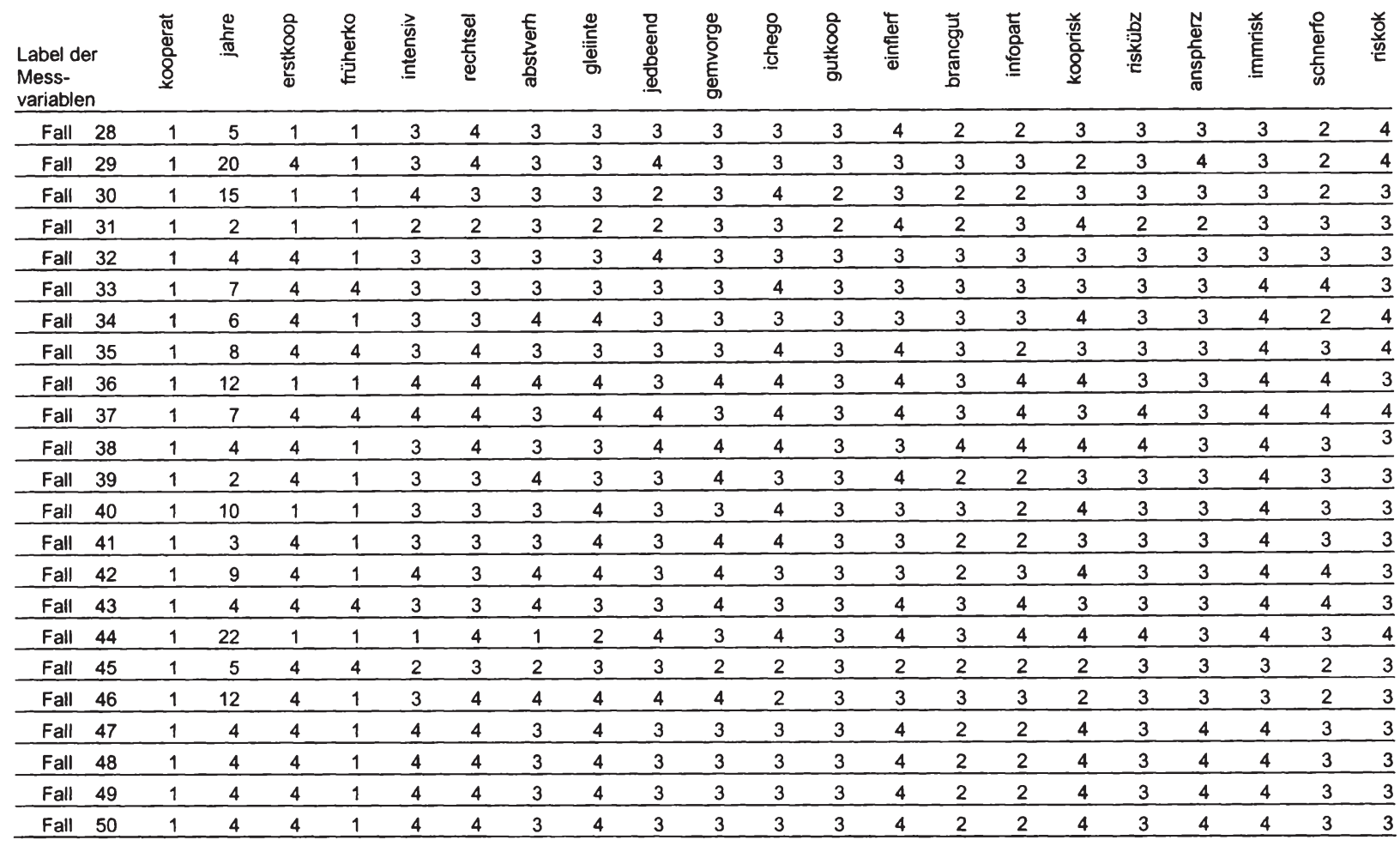




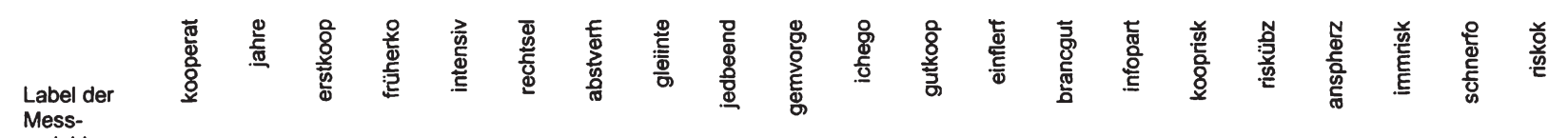

Mess-

variablen

\begin{tabular}{cllllllllllllllllllllllllll}
\hline Fall & 51 & 1 & 4 & 4 & 1 & 4 & 4 & 3 & 4 & 3 & 3 & 3 & 3 & 4 & 2 & 2 & 4 & 3 & 4 & 4 & 3 & 3 \\
\hline Fall & 52 & 1 & 4 & 4 & 1 & 4 & 4 & 3 & 4 & 3 & 3 & 3 & 3 & 4 & 2 & 2 & 4 & 3 & 4 & 4 & 3 & 3 \\
\hline Fall & 53 & 1 & 4 & 4 & 1 & 4 & 4 & 3 & 4 & 3 & 3 & 3 & 3 & 4 & 2 & 2 & 4 & 3 & 4 & 4 & 3 & 3 \\
\hline Fall & 54 & 1 & 4 & 4 & 1 & 4 & 4 & 3 & 4 & 3 & 3 & 3 & 3 & 4 & 2 & 2 & 4 & 3 & 4 & 4 & 3 & 3 \\
\hline Fall & 55 & 1 & 4 & 4 & 1 & 4 & 4 & 3 & 4 & 3 & 3 & 3 & 3 & 4 & 2 & 2 & 4 & 3 & 4 & 4 & 3 & 3 \\
\hline Fall & 56 & 1 & 4 & 4 & 1 & 4 & 4 & 3 & 4 & 3 & 3 & 3 & 3 & 4 & 2 & 2 & 4 & 3 & 4 & 4 & 3 & 3 \\
\hline Fall & 57 & 1 & 4 & 4 & 1 & 4 & 4 & 3 & 4 & 3 & 3 & 3 & 3 & 4 & 2 & 2 & 4 & 3 & 4 & 4 & 3 & 3 \\
\hline
\end{tabular}

Fall $58 \quad 0$,

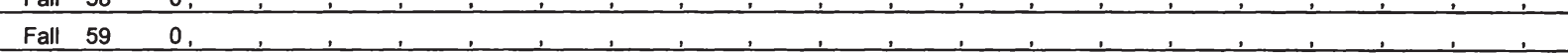

Fall $60 \quad 0$,

Fall $61 \quad 0$,

Fall $62 \quad 0$,

Fall $63 \quad 0$,

Fall $64 \quad 0$,

Fall 650 ,

Fall $66 \quad 0$,

Fall $67 \quad 0$,

Fall $68 \quad 0$,

Fall $69 \quad 0$,

Fall $70 \quad 0$,

Fall $71 \quad 0$,

Fall $72 \quad 0$

Fall $73 \quad 0$ 


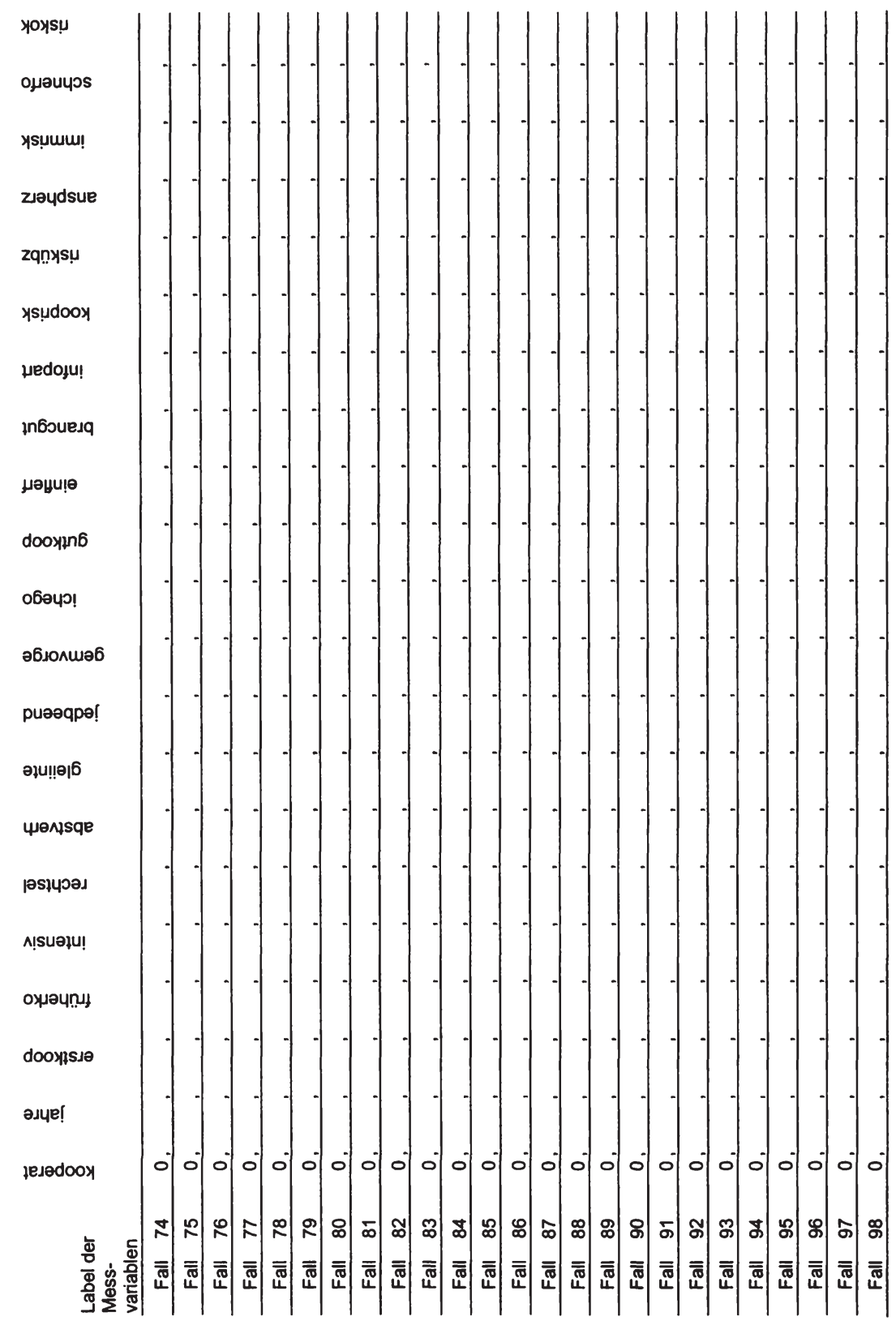




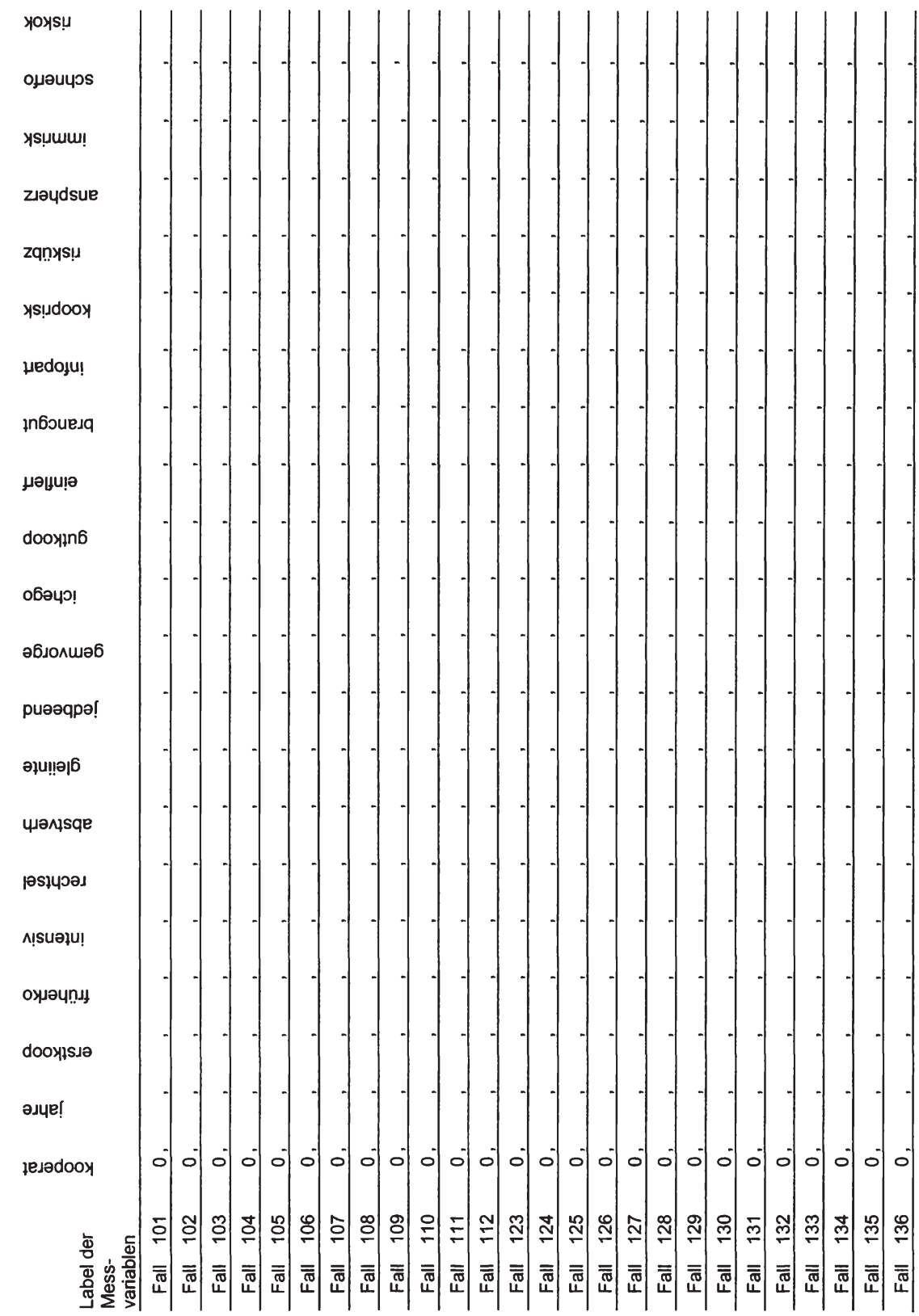




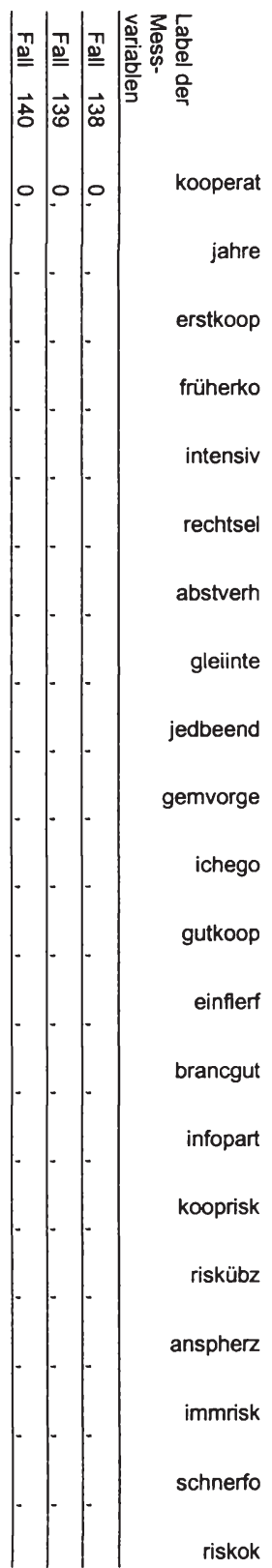




\begin{tabular}{|c|c|c|c|c|c|c|c|c|c|c|c|c|c|c|c|c|c|c|c|c|c|c|}
\hline $\begin{array}{l}\text { Label de } \\
\text { Mess- } \\
\text { variabler }\end{array}$ & & 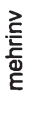 & $\begin{array}{l}\text { 음 } \\
\text { 을 } \\
\text { 톡 }\end{array}$ & $\frac{2}{\frac{2}{2}}$ & 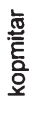 & $\begin{array}{l}\text { 밓 } \\
\frac{0}{0} \\
\frac{0}{0}\end{array}$ & $\begin{array}{l}\text { 등 } \\
\text { 홓 } \\
\text { 음 }\end{array}$ & 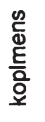 & 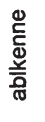 & $\begin{array}{l}\text { 윰 } \\
\text { 突 } \\
\text { 욛 }\end{array}$ & 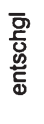 & 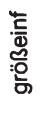 & 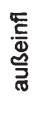 & 姜 & $\frac{\text { 응 }}{\frac{0}{0}}$ & 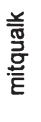 & $\begin{array}{l}\frac{0}{8} \\
\frac{1}{0} \\
\frac{0}{6}\end{array}$ & $\begin{array}{l}\text { 응 } \\
\text { 힌 } \\
\text { 응 }\end{array}$ & $\begin{array}{l}\text { 등 } \\
\text { \& } \\
\text { \&্刃 }\end{array}$ & $\begin{array}{l}\text { 름 } \\
\text { 들 } \\
\text { E⿱ } \\
\text { E }\end{array}$ & 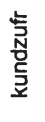 & 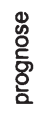 \\
\hline Fall & 1 & 2 & 2 & 4 & 1 & 3 & 4 & 2 & 3 & 3 & 2 & 2 & 1 & 3 & 2 & 4 & 4 & 3 & 2 & & 4. & \\
\hline Fall & 2 & 2 & 3 & 3 & 1 & 3 & 4 & 3 & 3 & 4 & 4 & 3 & 4 & 3 & 3 & 3 & 3 & 3 & 3 & 4 & 2 & 3 \\
\hline Fall & 3 & 3 & 3 & 3 & 1 & 3 & 2 & 3 & 3 & 3 & 2 & 3 & 2 & 3 & 3 & 3 & 3 & 3 & 3 & 4 & 3 & 3 \\
\hline Fall & 4 & 3 & 4 & 4 & 4 & 3 & 1 & 4 & 3 & 3 & 2 & 3 & 2 & 3 & 3 & 3 & 3 & 3 & 3 & 4 & 3 & 3 \\
\hline Fall & 5 & 2 & 3 & 2 & 1 & 4 & 1 & 3 & 3 & 4 & 3 & 2 & 3 & 3 & 3 & 3 & 3 & 4 & 3 & 4 & 4 & 4 \\
\hline Fall & 6 & 3 & 3 & 3 & 4 & 4 & 2 & 3 & 3 & 3 & 3 & 2 & 3 & 3 & 3 & 2 & 3 & 3 & 4 & 4 & 3 & 3 \\
\hline Fall & 7 & 3 & 3 & 3 & 1 & 3 & 3 & 4 & 3 & 3 & 3 & 3 & 3 , & & 3 & 3 & 3 & 3 & 3 & 4 & 3 & 4 \\
\hline Fall & 8 & 2 & 3 & 3 & 1 & 3 & 3 & 3 & 3 & 3 & 3 & 3 & 3 & 3 & 3 & 2 & 3 & 3 & 2 & 4 & 3 & 3 \\
\hline Fall & 9 & 3 & 3 & 3 & 4 & 3 & 2 & 3 & 3 & 3 & 3 & 3 & 3 & 3 & 3 & 3 & 3 & 3 & 2 & 4 & 4 & 3 \\
\hline Fall & 10 & 3 & 4 & 4 & 1 & 2 & 2 & 2 & 2 & 3 & 3 & 3 & 3 & 2 & 2 & 2 & 3 & 3 & 3 & 4 & 3 & 3 \\
\hline Fall & 11 & 2 & 3 & 3 & 1 & 3 & 3 & 3 & 3 & 3 & 3 & 2 & 3 & 2 & 3 & 2 & 3 & 3 & 3 & 4 & 4 & 4 \\
\hline Fall & 12 & 2 & 3 & 3 & 1 & 3 & 2 & 3 & 3 & 3 & 3 & 3 & 3 & 2 & 3 & 3 & 3 & 3 & 3 & 1 & 3 & 3 \\
\hline Fall & 13 & 3 & 4 & 4. & & 2 & 2 & 3 & 3 & 3 & 3 & 3 & 2 & 2 & 2 & 2 & 3 & 3 & 3 & 1 & 3 & 3 \\
\hline Fall & 14 & 2 & 3 & 2 & 1 & 2 & 2 & 3 & 3 & 3 & 4 & 2 & 4 & 3 & 4 & 2 & 3 & 2 & 3 & 1 & 3 & 3 \\
\hline Fall & 15 & 2 & 3 & 3 & 1 & 3 & 3 & 2 & 4 & 3 & 2 & 2 & 2 & 2 & 3 & 2 & 3 & 3 & 3 & 4 & 2 & 2 \\
\hline Fall & 16 & 2 & 3 & 3 & 4 & 3 & 3 & 3 & 2 & 3 & 3 & 3 & 2 & 2 & 3 & 2 & 3 & 3 & 3 & 4 & 4 & 3 \\
\hline Fall & 17 & 4 & 3 & 3 & 4 & 4 & 2 & 3 & 3 & 3 & 4 & 1 & 4 & 2 & 4 & 3 & 4 & 4 & 3 & 4 & 4 & 4 \\
\hline Fall & 18 & 2 & 3 & 2 & 1 & 3 & 3 & 3 & 2 & 3 & 2 & 2 & 3 & 3 & 3 & 2 & 2 & 3 & 3 & 4 & 3 & 3 \\
\hline Fall & 19 & 2 & 3 & 3 & 1 & 3 & 2 & 4 & 3 & 3 & 3 & 3 & 3 & 3 & 3 & 2 & 3 & 4 & 3 & 4 & 3 & 3 \\
\hline Fall & 20 & 3 & 3 & 3 & 1 & 2 & 2 & 4 & 3 & 3 & 2 & 2 & 1 & 3 & 3 & 3 & 2 & 3 & 3 & 4 & 2 & 3 \\
\hline Fall & 21 & 3 & 3 & 3 & 1 & 3 & 2 & 3 & 3 & 3 & 3 & 3 & 3 & 2 & 3 & 3 & 3 & 3 & 3 & 4 & 3 & 3 \\
\hline Fall & 22 & 3 & 3 & 3 & 1 & 4 & 3 & 3 & 3 & 3 & 3 & 3 & 3 & 3 & 3 & 3 & 4 & 4 & 4 & 4 & 3 & 3 \\
\hline Fall & 23 & 2 & 3 & 3 & 1 & 3 & 2 & 3 & 3 & 3 & 3 & 2 & 3 & 3 & 3 & 3 & 2 & 2 & 2 & 4 & 3 & 3 \\
\hline Fall & 24 & 3 & 1 & 3 & 4 & 3 & 4 & 3 & 4 & 3 & 3 & 4 & 4 & 2 & 4 & 3 & 3 & 4 & 3 & 4 & 4 & 3 \\
\hline Fall & 25 & 2 & 3 & 3 & 4 & 3 & 3 & 2 & 3 & 3 & 4 & 3 & 4 & 1 & 3 & 2 & 3 & 3 & 3 & 4 & 3 & 3 \\
\hline Fall & 26 & 3 & 4 & 4 & 4 & 2 & 1 & 2 & 3 & 3 & 4 & 4 & 3 & 1 & 3 & 3 & 3 & 3 & 4 & 4 & 4 & 3 \\
\hline Fall & 27 & 2 & 2 & 3 & 1 & 3 & 3 & 4 & 3 & 3 & 3 & 2 & 2 & 3 & 3 & 3 & 3 & 3 & 3 & 1 & 4 & 3 \\
\hline
\end{tabular}




\begin{tabular}{|c|c|c|c|c|c|c|c|c|c|c|c|c|c|c|c|c|c|c|c|c|c|}
\hline $\begin{array}{l}\text { Label der } \\
\text { Mess- } \\
\text { variablen }\end{array}$ & 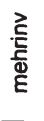 & $\begin{array}{l}\frac{0}{3} \\
\frac{\bar{a}}{0} \\
\frac{5}{5}\end{array}$ & $\frac{2}{\frac{2}{2}}$ & 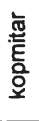 & 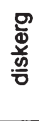 & 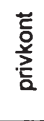 & $\begin{array}{l}\stackrel{0}{0} \\
\Phi \\
\frac{E}{2} \\
\stackrel{0}{2}\end{array}$ & 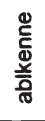 & 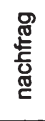 & 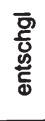 & 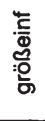 & 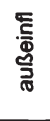 & 羔 & 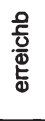 & 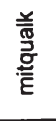 & $\begin{array}{l}\frac{8}{8} \\
\text { के } \\
\frac{10}{\infty}\end{array}$ & $\begin{array}{l}\overline{0} \\
\text { 흘 } \\
\text { o }\end{array}$ & $\begin{array}{l}\text { 总 } \\
\text { \% } \\
\end{array}$ & $\begin{array}{l}\text { 글 } \\
\stackrel{\bar{Z}}{\bar{E}} \\
\text { ह๊ }\end{array}$ & $\begin{array}{l}\text { 点 } \\
\text { N } \\
\frac{5}{5} \\
\underline{\underline{y}}\end{array}$ & 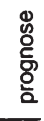 \\
\hline Fall 29 & 3 & 4 & 3 & 1 & 2 & 2 & 3 & 3 & 3 & 3 & 2 & 2 & 2 & 3 & 2 & 3 & 3 & 3 & 4 & 3 & 3 \\
\hline Fall 30 & 2 & 2 & 2 & 4 & 3 & 3 & 3 & 3 & 3 & 2 & 2 & 2 & 2 & 3 & 3 & 3 & 3 & 3 & 4 & 4 & 3 \\
\hline Fall 31 & 3 & 2 & 2 & 1 & 2 & 1 & 1 & 2 & 2 & 2 & 3 & 2 & 2 & 2 & 2 & 2 & 2 & 3 & 4 & 3 & 3 \\
\hline Fall 32 & 2 & 2 & 2 & 1 & 2 & 2 & 3 & 3 & 3 & 4 & 4 & 3 & 3 & 3 & 2 & 3 & 3 & 4 & 4 & 3 & 3 \\
\hline Fall 33 & 2 & 3 & 3 & 4 & 2 & 3 & 2 & 4 & 4 & 3 & 3 & 4 & 2 & 3 & 3 & 3 & 3 & 3 & 4 & 4 & 3 \\
\hline Fall 34 & 3 & 3 & 3 & 4 & 2 & 2 & 3 & 3 & 3 & 4 & 4 & 3 & 3 & 3 & 3 & 2 & 3 & 4 & 4 & 4 & 3 \\
\hline Fall 35 & 2 & 3 & 4 & 1 & 3 & 2 & 3 & 3 & 4 & 3 & 3 & 3 & 3 & 3 & 3 & 3 & 3 & 4 & 4 & 3 & 3 \\
\hline Fall 36 & 3 & 4 & 4 & 4 & 2 & 3 & 3 & 4 & 4 & 4 & 4 & 3 & 2 & 3 & 3 & 3 & 3 & 4 & 4 & 3 & 3 \\
\hline Fall 37 & 3 & 3 & 3 & 1 & 3 & 3 & 3 & 4 & 3 & 3 & 3 & 4 & 3 & 3 & 2 & 4 & 4 & 3 & 4 & 3 & 3 \\
\hline Fall 38 & 3 & 3 & 3 & 1 & 3 & 3 & 1 & 3 & 3 & 3 & 1 & 4 & 2 & 3 & 3 & 2 & 3 & 4 & 4 & 3 & 3 \\
\hline Fall 39 & 2 & 3 & 3 & 1 & 3 & 2 & 4 & 3 & 3 & 4 & 3 & 3 & 3 & 3 & 3 & 3 & 3 & 3 & 4 & 3 & 3 \\
\hline Fall 40 & 3 & 4 & 4 & 4 & 3 & 2 & 3 & 3 & 4 & 4 & 3 & 3 & 3 & 3 & 3 & 3 & 3 & 4 & 4 & 3 & 3 \\
\hline Fall 41 & 3 & 3 & 3 & 1 & 2 & 2 & 2 & 3 & 3 & 4 & 3 & 4 & 2 & 3 & 3 & 3 & 3 & 4 & 4 & 3 & 3 \\
\hline Fall 42 & 3 & 3 & 3 & 4 & 2 & 3 & 3 & 3 & 3 & 4 & 4 & 3 & 3 & 3 & 2 & 4 & 3 & 3 & 4 & 3 & 3 \\
\hline Fall 43 & 2 & 3 & 3 & 4 & 3 & 2 & 3 & 3 & 3 & 3 & 3 & 3 & 3 & 2 & 3 & 2 & 3 & 3 & 4 & 3 & 3 \\
\hline Fall 44 & 3 & 3 & 3 & 1 & 3 & 3 & 1 & 4 & 2 & 1 & 4 & 2 & 1 & 3 & 4 & 4 & 3 & 4 & 1 & 2 & 2 \\
\hline Fall 45 & 2 & 2 & 2 & 1 & 3 & 2 & 2 & 2 & 3 & 2 & 3 & 3 & 3 & 3 & 2 & 2 & 2 & 2 & 1 & 3 & 3 \\
\hline Fall 46 & 2 & 3 & 3 & 1 & 3 & 3 & 3 & 3 & 2 & 3 & 3 & 3 & 2 & 3 & 2 & 3 & 3 & 2 & 4 & 4 & 2 \\
\hline Fall 47 & 3 & 3 & 3 & 1 & 3 & 2 & 4 & 3 & 3 & 4 & 4 & 4 & 3 & 3 & 3 & 3 & 4 & 3 & 4 & 4 & 3 \\
\hline Fall 48 & 3 & 3 & 3 & 1 & 3 & 2 & 4 & 3 & 3 & 4 & 4 & 4 & 3 & 3 & 3 & 3 & 4 & 3 & 4 & 4 & 3 \\
\hline Fall 49 & 3 & 3 & 3 & 1 & 3 & 2 & 4 & 3 & 3 & 4 & 4 & 4 & 3 & 3 & 3 & 3 & 4 & 3 & 4 & 4 & 3 \\
\hline Fall 50 & 3 & 3 & 3 & 1 & 3 & 2 & 4 & 3 & 3 & 4 & 4 & 4 & 3 & 3 & 3 & 3 & 4 & 3 & 4 & 4 & 3 \\
\hline Fall 51 & 3 & 3 & 3 & 1 & 3 & 2 & 4 & 3 & 3 & 4 & 4 & 4 & 3 & 3 & 3 & 3 & 4 & 3 & 4 & 4 & 3 \\
\hline
\end{tabular}




\begin{tabular}{|c|c|c|c|c|c|c|c|c|c|c|c|c|c|c|c|c|c|c|c|c|c|c|}
\hline $\begin{array}{l}\text { Label d } \\
\text { Mess- } \\
\text { variable }\end{array}$ & & 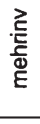 & $\begin{array}{l}\text { 몽 } \\
\text { 흠 } \\
\text { है }\end{array}$ & $\frac{n}{2}$ & 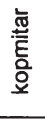 & $\begin{array}{l}\text { 口্ } \\
\frac{0}{0} \\
\frac{5}{0}\end{array}$ & $\begin{array}{l}\text { 든 } \\
\text { 몸 } \\
\text { 름 }\end{array}$ & 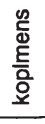 & 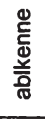 & 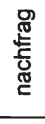 & 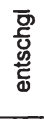 & 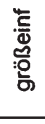 & 突 & 羔 & $\begin{array}{l}\frac{}{\frac{1}{0}} \\
\frac{0}{0} \\
\frac{1}{0}\end{array}$ & $\begin{array}{l}\text { 差 } \\
\text { 言 } \\
\text { E }\end{array}$ & $\begin{array}{l}\text { 응 } \\
\frac{8}{0} \\
\text { 흥 }\end{array}$ & $\begin{array}{l}\text { 증 } \\
\text { 힌 } \\
\text { 응 }\end{array}$ & $\begin{array}{l}\text { 등 } \\
\text { : } \\
\text { 양 }\end{array}$ & $\begin{array}{l}\text { 름 } \\
\text { 듬 } \\
\text { 른 }\end{array}$ & 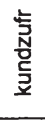 & 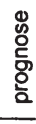 \\
\hline Fall & 53 & 3 & 3 & 3 & 1 & 3 & 2 & 4 & 3 & 3 & 4 & 4 & 4 & 3 & 3 & 3 & 3 & 4 & 3 & 4 & 4 & 3 \\
\hline Fall & 54 & 3 & 3 & 3 & 1 & 3 & 2 & 4 & 3 & 3 & 4 & 4 & 4 & 3 & 3 & 3 & 3 & 4 & 3 & 4 & 4 & 3 \\
\hline Fall & 56 & 3 & 3 & 3 & 1 & 3 & 2 & 4 & 3 & 3 & 4 & 4 & 4 & 3 & 3 & 3 & 3 & 4 & 3 & 4 & 4 & 3 \\
\hline Fall & 58, & & & & & & & & & & & & & & & & & & & & 3 & 3 \\
\hline Fall & 59, & & & & & & 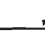 & & & & & & & & & & & & & & 3 & 3 \\
\hline Fall & 60 , & & & & & & & & & & & & & & & . & & , & & & 4 & $\underline{2}$ \\
\hline Fall & 61, & & 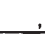 & 1 & &. & 1 & & & & - & -4 & - & - & 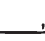 & 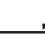 & & 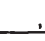 & 1 & & 3 & 3 \\
\hline Fall & 62 , & & 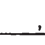 & 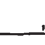 & & T. & W & 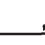 & . & & & - & - & 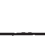 & 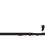 & , & & , & & & 4 & 3 \\
\hline Fall & 63 , & & & 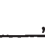 & & 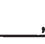 & 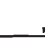 & & & & & & & & & 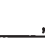 & & 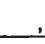 & & & 4 & 3 \\
\hline Fall & 65, & & 1 & & & 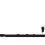 & ـ & & & & & & & & 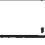 & . & & , & & & 4 & 3 \\
\hline Fall & 66, & & . & & & 1 & & & & & & & & & & & & 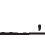 & & & 3 & 3 \\
\hline Fall & 67 , & & , & & & & & & & & & & & & & . & & , & & & 3 & 3 \\
\hline Fall & 68 , & & , & & , & , & . & & & & & & & & & . & & , & & & 4 & 4 \\
\hline Fall & 69 , & & . & , & & , & & & & & & & & & & & & , & & & 3 & 3 \\
\hline Fall & 70, & & & & & & & & & & & & & & & & & & & & & 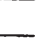 \\
\hline Fall & 71 , & & 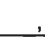 & & & & & & & & & & & & & , & & 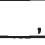 & & & 3 & 3 \\
\hline Fall & 72 , & & & & & & . & & & & & & & & & & & - & & & 3 & 3 \\
\hline Fall & 73 . & & & & & & & & & & & & & & 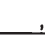 & & & 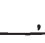 & 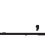 & & 4 & 3 \\
\hline Fall & 74, & & 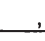 & & & & 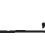 & & & & & & & & & 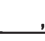 & . & 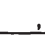 & & & 3 & 2 \\
\hline Fall & 75 , & & & & & 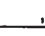 & 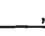 & & & & & & & & & & & . & & & 3 & 3 \\
\hline
\end{tabular}




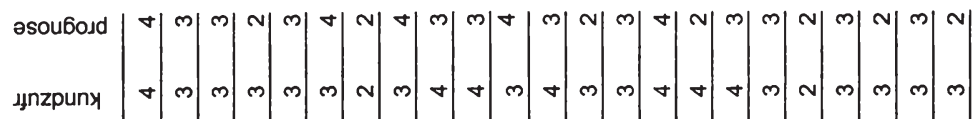

n|dpuew

צบบรัธ6ว

1qosdıds

dooyəuds

y|enbỵu

qบวฺอมว

ㄴำฺบำ

ษu!̣gne

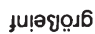

|ธิบอร\}บ

бедуреи

әuนə习习|qe

suəu|doy

juoynu!d

Бృәуร!р

sepludox

snjdıs

sn|dswn

миบำ

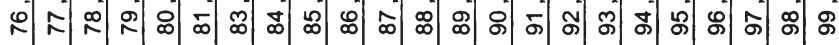

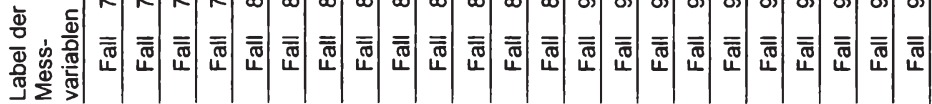




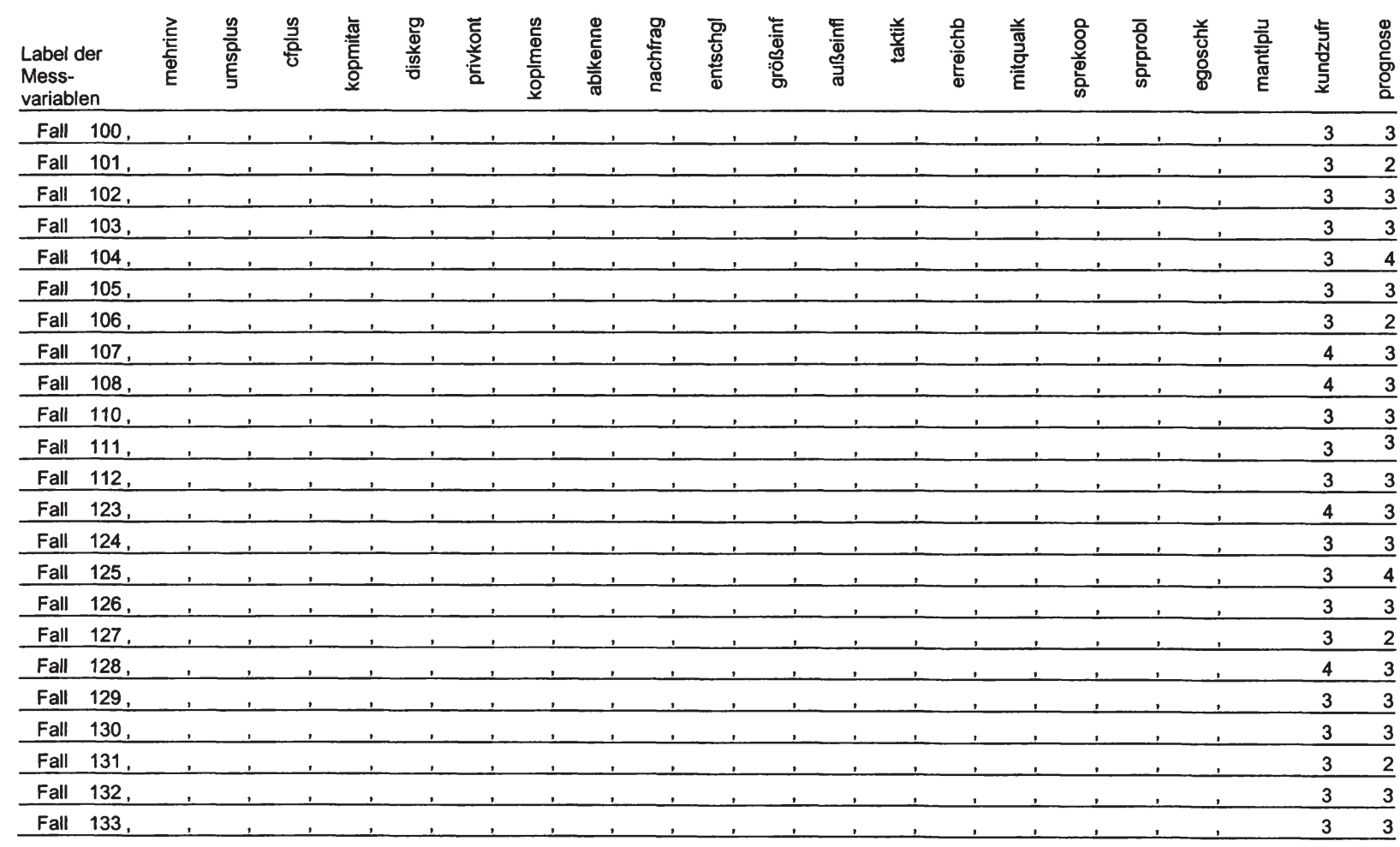




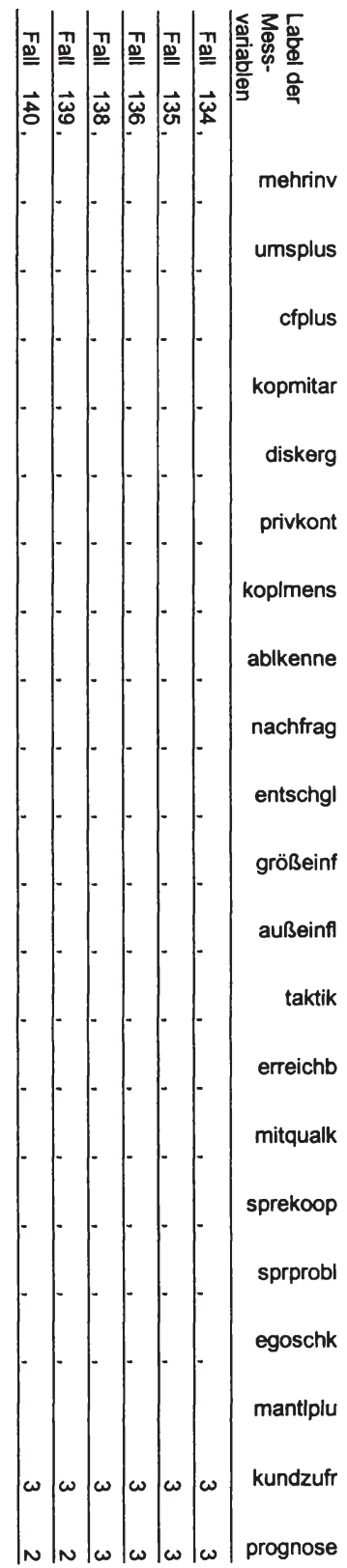




\begin{tabular}{|c|c|c|c|c|c|c|c|c|c|c|}
\hline $\begin{array}{l}\text { Label de } \\
\text { Mess- } \\
\text { variabler }\end{array}$ & & $\frac{3}{3}$ & $\frac{\text { 금 }}{\text { ํㅡㅁ }}$ & $\begin{array}{l}\frac{3}{\frac{3}{2}} \\
\frac{2}{\mathfrak{*}} \\
\varepsilon\end{array}$ & 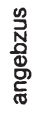 & 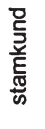 & $\begin{array}{l}\frac{\bar{\Phi}}{\mathrm{E}} \\
\frac{\mathrm{J}}{\omega}\end{array}$ & $\begin{array}{l}\stackrel{\mathscr{T}}{J} \\
\stackrel{0}{0} \\
\text { E }\end{array}$ & $\frac{\stackrel{M}{E}}{\stackrel{\mathscr{E}}{\Phi}}$ & 胥 \\
\hline Fall & 1 & 4 & 3 & 3 & 2 & 3 & 3 & 4 & 1 & 1 \\
\hline Fall & 2 & 3 & 3 & 3 & 2 & 3 & 3 & 3 & 4 & 4 \\
\hline Fall & 3 & 3 & 3 & 3 & 2 & 3 & 3 & 3 & 4 & 4 \\
\hline Fall & 4 & 3 & 3 & 3 & 3 & 4 & 4 & 3 & 4 & 4 \\
\hline Fall & 5 & 3 & 3 & 3 & 1 & 4 & 4 & 3 & 4 & 4 \\
\hline Fall & 6 & 3 & 3 & 3 & 3 & 2 & 4 & 2 & 4 & 4 \\
\hline Fall & 7 & 4 & 4 & 4 & 2 & 4 & 3 & 3 & 4 & 4 \\
\hline Fall & 8 & 2 & 2 & 2 & 2 & 4 & 4 & 1 & 4 & 1 \\
\hline Fall & 9 & 2 & 2 & 2 & 2 & 4 & 2 & 3 & 4 & 4 \\
\hline Fall & 10 & 3 & 3 & 3 & 1 & 2 & 2 & 2 & 4 & 1 \\
\hline Fall & 11 & 4 & 4 & 4 & 1 & 3 & 4 & 4 & 4 & 4 \\
\hline Fall & 12 & 3 & 3 & 3 & 2 & 2 & 3 & 3 & 1 & 1 \\
\hline Fall & 13 & 3 & 3 & 2 & 2 & 4 & 4 & 3 & 4 & 4 \\
\hline Fall & 14 & 2 & 3 & 2 & 2 & 3 & 3 & 3 & 4 & 1 \\
\hline Fall & 15 & 3 & 3 & 3 & 2 & 3 & 3 & 2 & 4 & 4 \\
\hline Fall & 16 & 3 & 2 & 3 & 2 & 4 & 4 & 3 & 4 & 4 \\
\hline Fall & 17 & 3 & 4 & 4 & 3 & 4 & 4 & 4 & 4 & 4 \\
\hline Fall & 18 & 3 & 3 & 3 & 3 & 3 & 3 & 3 & 4 & 4 \\
\hline Fall & 19 & 3 & 3 & 3 & 3 & 3 & 3 & 3 & 4 & 4 \\
\hline Fall & 20 & 2 & 3 & 2 & 3 & 3 & 3 & 3 & 1 & 4 \\
\hline Fall & 21 & 3 & 3 & 3 & 1 & 3 & 3 & 3 & 4 & 4 \\
\hline Fall & 22 & 3 & 3 & 3 & 2 & 3 & 3 & 4 & 4 & 4 \\
\hline Fall & 23 & 3 & 3 & 3 & 2 & 3 & 3 & 3 & 4 & 4 \\
\hline Fall & 24 & 4 & 3 & 2 & 3 & 3 & 3 & 3 & 1 & 4 \\
\hline Fall & 25 & 3 & 3 & 3 & 3 & 3 & 3 & 3 & 4 & 4 \\
\hline Fall & 26 & 3 & 3 & 3 & 2 & 3 & 3 & 1 & 4 & 4 \\
\hline Fall & 27 & 3 & 2 & 2 & 1 & 3 & 3 & 3 & 1 & 1 \\
\hline
\end{tabular}




\begin{tabular}{|c|c|c|c|c|c|c|c|c|c|c|}
\hline $\begin{array}{l}\text { Label d } \\
\text { Mess- } \\
\text { variable }\end{array}$ & & $\frac{m}{30}$ & $\begin{array}{l}\text { 马 } \\
\text { 믐 } \\
\text { है } \\
\text { है }\end{array}$ & $\begin{array}{l}\text { ᄁ } \\
\frac{\overline{0}}{\frac{1}{0}} \\
\text { है }\end{array}$ & $\begin{array}{l}\text { N } \\
\text { N } \\
\text { D } \\
\text { D్ }\end{array}$ & 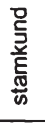 & 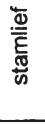 & 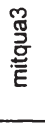 & $\begin{array}{l}\stackrel{\mathscr{M}}{E} \\
\stackrel{\underline{D}}{E}\end{array}$ & $\begin{array}{l}\stackrel{M}{\complement} \\
\stackrel{\text { D }}{\Phi} \\
\text { E }\end{array}$ \\
\hline Fall & 28 & 2 & 3 & 2 & 1 & 4 & 3 & 3 & 4 & 1 \\
\hline Fall & 29 & 2 & 3 & 3 & 2 & 4 & 4 & 2 & 4 & 4 \\
\hline Fall & 30 & 3 & 3 & 3 & 1 & 3 & 3 & 3 & 4 & 4 \\
\hline Fall & 31 & 2 & 2 & 3 & 2 & 3 & 2 & 2 & 1 & 4 \\
\hline Fall & 32 & 3 & 3 & 3 & 1 & 2 & 3 & 3 & 4 & 4 \\
\hline Fall & 33 & 2 & 2 & 3 & 2 & 2 & 3 & 3 & 4 & 4 \\
\hline Fall & 34 & 3 & 3 & 3 & 2 & 3 & 3 & 3 & 4 & 1 \\
\hline Fall & 35 & 3 & 4 & 3 & 3 & 3 & 3 & 3 & 4 & 4 \\
\hline Fall & 36 & 3 & 3 & 3 & 3 & 4 & 4 & 3 & 4 & 4 \\
\hline Fall & 37 & 3 & 3 & 3 & 3 & 4 & 4 & 2 & 4 & 4 \\
\hline Fall & 38 & 3 & 3 & 3 & 3 & 3 & 4 & 3 & 1 & 4 \\
\hline Fall & 39 & 3 & 3 & 3 & 2 & 4 & 4 & 3 & 4 & 4 \\
\hline Fall & 40 & 4 & 4 & 4 & 3 & 3 & 3 & 3 & 4 & 4 \\
\hline Fall & 41 & 2 & 2 & 2 & 2 & 3 & 3 & 2 & 4 & 4 \\
\hline Fall & 42 & 3 & 3 & 3 & 2 & 4 & 3 & 2 & 4 & 4 \\
\hline Fall & 43 & 3 & 3 & 3 & 3 & 3 & 3 & 3 & 4 & 1 \\
\hline Fall & 44 & 3 & 3 & 3 & 1 & 4 & 2 & 1 & 1 & 1 \\
\hline Fall & 45 & 2 & 2 & 2 & 2 & 3 & 3 & 2 & 4 & 1 \\
\hline Fall & 46 & 3 & 3 & 3 & 2 & 3 & 3 & 2 & 4 & 1 \\
\hline Fall & 47 & 3 & 3 & 3 & 1 & 3 & 3 & 3 & 4 & 4 \\
\hline Fall & 48 & 3 & 3 & 3 & 1 & 3 & 3 & 3 & 4 & 4 \\
\hline Fall & 49 & 3 & 3 & 3 & 1 & 3 & 3 & 3 & 4 & 4 \\
\hline Fall & 50 & 3 & 3 & 3 & 1 & 3 & 3 & 3 & 4 & 4 \\
\hline
\end{tabular}




\begin{tabular}{|c|c|c|c|c|c|c|c|c|c|c|}
\hline \multicolumn{2}{|c|}{$\begin{array}{l}\text { Label der } \\
\text { Mess- } \\
\text { variablen }\end{array}$} & \multirow{2}{*}{$\frac{\frac{3}{3}}{\frac{3}{0}}$} & \multirow{2}{*}{ 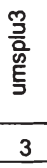 } & \multirow{2}{*}{$\begin{array}{c}\frac{\text { M }}{\bar{z}} \\
\text { 亭 } \\
\text { E. }\end{array}$} & \multirow{2}{*}{ 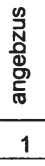 } & \multirow{2}{*}{ 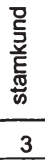 } & \multirow{2}{*}{ 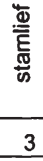 } & \multirow{2}{*}{ 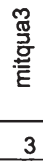 } & \multirow{2}{*}{ 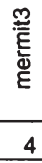 } & \multirow{2}{*}{ 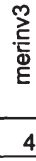 } \\
\hline Fall & 51 & & & & & & & & & \\
\hline Fall & 52 & 3 & 3 & 3 & 1 & 3 & 3 & 3 & 4 & 4 \\
\hline Fall & 53 & 3 & 3 & 3 & 1 & 3 & 3 & 3 & 4 & 4 \\
\hline Fall & 54 & 3 & 3 & 3 & 1 & 3 & 3 & 3 & 4 & 4 \\
\hline Fall & 55 & 3 & 3 & 3 & 1 & 3 & 3 & 3 & 4 & 4 \\
\hline Fall & 56 & 3 & 3 & 3 & 1 & 3 & 3 & 3 & 4 & 4 \\
\hline Fall & 57 & 3 & 3 & 3 & 1 & 3 & 3 & 3 & 4 & 4 \\
\hline Fall & 58 & 3 & 4 & 3 & 2 & 2 & 2 & 3 & 4 & 1 \\
\hline Fall & 59 & 4 & 4 & 4 & 2 & 3 & 3 & 4 & 4 & 1 \\
\hline Fall & 60 & 2 & 2 & 2 & 1 & 3 & 4 & 2 & 4 & 1 \\
\hline Fall & 61, & & 1 & 2 & 2 & 3 & 3 & 3 & 4 & 4 \\
\hline Fall & 62 & 3 & 3 & 3 & 2 & 3 & 2 & 3 & 4 & 1 \\
\hline Fall & 63 & 2 & 2 & 2 & 2 & 2 & 3 & 3 & 4 & 1 \\
\hline Fall & 64 & 2 & 2 & 2 & 3 & 3 & 3 & 3 & 1 & 4 \\
\hline Fall & 65 & 2 & 2 & 2 & 1 & 4 & 4 & 3 & 4 & 1 \\
\hline Fall & 66 & 3 & 2 & 2 & 3 & 2 & 3 & 3 & 4 & 1 \\
\hline Fall & 67 & 4 & 4 & 4 & 3 & 2 & 3 & 3 & 4 & 1 \\
\hline Fall & 68 & 3 & 2 & 3 & 1 & 3 & 4 & 3 & 4 & 1 \\
\hline Fall & 69 & 2 & 1 & 2 & 3 & 3 & 4 & 3 & 4 & 1 \\
\hline Fall & 70 & 3 & 3 & 3 & 1 & 2 & 3 & 3 & 4 & 1 \\
\hline Fall & 71 & 3 & 3 & 3 & 3 & 3 & 3 & 3 & 4 & 4 \\
\hline Fall & 72 & 2 & 2 & 2 & 2 & 3 & 3 & 3 & 1 & 4 \\
\hline Fall & 73 & 2 & 2 & 2 & 2 & 4 & 4 & 3 & 4 & 1 \\
\hline
\end{tabular}




\begin{tabular}{|c|c|c|c|c|c|c|c|c|c|c|}
\hline $\begin{array}{l}\text { Label d } \\
\text { Mess- } \\
\text { variable }\end{array}$ & & $\frac{3}{\frac{3}{a}}$ & 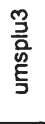 & 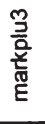 & 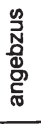 & 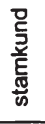 & 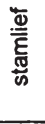 & 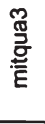 & $\stackrel{\stackrel{\mathscr{M}}{E}}{\underset{\Phi}{E}}$ & 茎 \\
\hline Fall & 74 & 2 & 2 & 2 & 1 & 2 & 3 & 2 & 4 & 4 \\
\hline Fall & 75 & 2 & 2 & 2 & 2 & 3 & 3 & 2 & 4 & 1 \\
\hline Fall & 76 & 4 & 4 & 4 & 3 & 4 & 4 & 3 & 4 & 4 \\
\hline Fall & 77 & 2 & 2 & 2 & 1 & 2 & 4 & 3 & 4 & 1 \\
\hline Fall & 78 & 4 & 4 & 3 & 1 & 2 & 3 & 3 & 4 & 1 \\
\hline Fall & 79 & 2 & 2 & 2 & 2 & 3 & 3 & 3 & 4 & 4 \\
\hline Fall & 80 & 3 & 3 & 3 & 2 & 2 & 2 & 3 & 4 & 4 \\
\hline Fall & 81 & 4 & 3 & 3 & 4 & 2 & 3 & 3 & 4 & 1 \\
\hline Fall & 82 & 3 & 2 & 2 & 1 & 3 & 3 & 4 & 4 & 1 \\
\hline Fall & 83 & 2 & 2 & 2 & 2 & 3 & 3 & 3 & 4 & 4 \\
\hline Fall & 84 & 2 & 1 & 2 & 2 & 4 & 2 & 2 & 4 & 4 \\
\hline Fall & 85 & 3 & 2 & 2 & 1 & 4 & 3 & 4 & 4 & 1 \\
\hline Fall & 86 & 2 & 2 & 2 & 1 & 2 & 3 & 2 & 4 & 1 \\
\hline Fall & 87 & 4 & 3 & 3 & 2 & 2 & 3 & 3 & 4 & 4 \\
\hline Fall & 88 & 2 & 2 & 2 & 1 & 4 & 4 & 3 & 1 & 4 \\
\hline Fall & 89 & 2 & 2 & 2 & 3 & 4 & 3 & 2 & 4 & 1 \\
\hline Fall & 90 & 4 & 4 & 3 & 2 & 3 & 3 & 3 & 1 & 1 \\
\hline Fall & 91 & 4 & 4 & 3 & 3 & 4 & 4 & 3 & 4 & 4 \\
\hline Fall & 92 & 2 & 2 & 2 & 2 & 4 & 4 & 3 & 4 & 1 \\
\hline Fall & 93 & 3 & 3 & 3 & 2 & 2 & 4 & 3 & 4 & 4 \\
\hline Fall & 94 & 2 & 2 & 1 & 2 & 2 & 2 & 2 & 1 & 1 \\
\hline Fall & 95 & 1 & 2 & 2 & 1 & 1 & 1 & 2 & 1 & 1 \\
\hline Fall & 96 & 2 & 2 & 2 & 1 & 2 & 3 & 2 & 4 & 1 \\
\hline
\end{tabular}




\begin{tabular}{|c|c|c|c|c|c|c|c|c|c|c|}
\hline \multicolumn{2}{|c|}{$\begin{array}{l}\text { Label der } \\
\text { Mess- } \\
\text { variablen }\end{array}$} & \multirow{2}{*}{$\frac{\frac{m}{3}}{\frac{\pi}{0}}$} & \multirow{2}{*}{$\begin{array}{l}\frac{3}{3} \\
\text { के } \\
\text { है }\end{array}$} & \multirow{2}{*}{$\begin{array}{c}\frac{M}{3} \\
\text { 帝 } \\
\text { है } \\
1\end{array}$} & \multirow{2}{*}{ 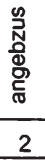 } & \multirow{2}{*}{ 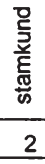 } & \multirow{2}{*}{ 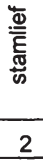 } & \multirow{2}{*}{ 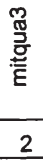 } & \multirow{2}{*}{$\begin{array}{r}\stackrel{\stackrel{\mathscr{m}}{\mathbf{E}}}{\mathbf{E}} \\
\\
\end{array}$} & \multirow{2}{*}{ 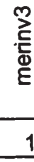 } \\
\hline Fall & 97 & & & & & & & & & \\
\hline Fall & 98 & 2 & 1 & 1 & 1 & 2 & 2 & 2 & 1 & 1 \\
\hline Fall & 99 & 2 & 2 & 2 & 1 & 2 & 2 & 2 & 1 & 4 \\
\hline Fall & 100 & 3 & 2 & 2 & 1 & 3 & 3 & 3 & 1 & 4 \\
\hline Fall & 101 & 1 & 1 & 2 & 1 & 2 & 2 & 3 & 1 & 1 \\
\hline Fall & 102 & 2 & 2 & 1 & 1 & 2 & 1 & 2 & 1 & 1 \\
\hline Fall & 103 & 4 & 4 & 4 & 2 & 1 & 3 & 3 & 4 & 4 \\
\hline Fall & 104 & 2 & 2 & 3 & 2 & 3 & 4 & 3 & 4 & 1 \\
\hline Fall & 105 & 3 & 3 & 3 & 1 & 3 & 3 & 3 & 4 & 4 \\
\hline Fall & 106 & 2 & 2 & 1 & 1 & 3 & 3 & 2 & 1 & 4 \\
\hline Fall & 107 & 2 & 3 & 3 & 2 & 4 & 4 & 4 & 4 & 4 \\
\hline Fall & 108 & 3 & 2 & 2 & 1 & 3 & 4 & 3 & 4 & 1 \\
\hline Fall & 109 & 2 & 2 & 2 & 2 & 4 & 4 & 3 & 4 & 1 \\
\hline Fall & 110 & 3 & 4 & 2 & 3 & 4 & 4 & 3 & 4 & 4 \\
\hline Fall & 111 & 3 & 3 & 3 & 2 & 2 & 3 & 4 & 4 & 4 \\
\hline Fall & 112 & 2 & 3 & 3 & 2 & 3 & 3 & 3 & 4 & 4 \\
\hline Fall & 123 & 2 & 2 & 2 & 1 & 4 & 4 & 3 & 4 & 4 \\
\hline Fall & 124 & 3 & 2 & 2 & 2 & 3 & 3 & 3 & 4 & 1 \\
\hline Fall & 125 & 4 & 3 & 2 & 3 & 3 & 3 & 3 & 4 & 1 \\
\hline Fall & 126 & 2 & 3 & 2 & 3 & 3 & 3 & 3 & 4 & 1 \\
\hline Fall & 127 & 2 & 1 & 1 & 1 & 3 & 3 & 2 & 1 & 1 \\
\hline Fall & 128 & 4 & 4 & 4 & 2 & 4 & 4 & 3 & 4 & 1 \\
\hline Fall & 129 & 1 & 1 & 1 & 1 & 2 & 2 & 2 & 1 & 1 \\
\hline
\end{tabular}




\begin{tabular}{|c|c|c|c|c|c|c|c|c|c|c|}
\hline $\begin{array}{l}\text { Label } \\
\text { Mess- } \\
\text { variab }\end{array}$ & & $\frac{\dddot{3}}{\frac{3}{0}}$ & $\begin{array}{l}\frac{m}{3} \\
\frac{3}{0} \\
\frac{1}{5}\end{array}$ & 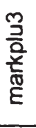 & 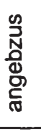 & 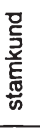 & 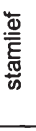 & 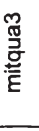 & 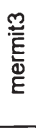 & 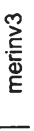 \\
\hline Fall & 130 & 2 & 2 & 2 & 2 & 2 & 1 & 2 & 4 & 1 \\
\hline Fall & 131 & 1 & 1 & 1 & 2 & 2 & 2 & 2 & 1 & 4 \\
\hline Fall & 132 & 2 & 2 & 2 & 2 & 2 & 2 & 2 & 1 & 1 \\
\hline Fall & 133 & 1 & 1 & 2 & 1 & 2 & 3 & 2 & 4 & 1 \\
\hline Fall & 134 & 2 & 2 & 2 & 2 & 2 & 2 & 2 & 4 & 1 \\
\hline Fall & 135 & 2 & 2 & 3 & 2 & 2 & 3 & 2 & 1 & 1 \\
\hline Fall & 136 & 2 & 2 & 2 & 1 & 3 & 3 & 2 & 4 & 1 \\
\hline Fall & 137 & 2 & 1 & 1 & 2 & 2 & 2 & 2 & 4 & 1 \\
\hline Fall & 138 & 1 & 1 & 2 & 1 & 3 & 2 & 2 & 4 & 1 \\
\hline Fall & 139 & 1 & 1 & 1 & 2 & 2 & 2 & 3 & 1 & 1 \\
\hline Fall & 140 & 2 & 2 & 1 & 1 & 2 & 3 & 2 & 1 & 1 \\
\hline
\end{tabular}



Appendix 3: Reliabilitätstest 


\section{Reliabilität - Itembatterie "Selbstverpflichtung"}

RELIABILITYANALYSIS-SCALE (SPLIT)

Reliability Coefficients

$\mathrm{N}$ of Cases $=55,0 \quad \mathrm{~N}$ of Items $=10$

Correlation between forms $=, 3219$ Equal-length Spearman-Brown $=, 4871$

Guttman Split-half $=, 4615 \quad$ Unequal-length Spearman-Brown $=, 4871$

5 Items in part $1 \quad 5$ Items in part 2

Alpha for part $1=, 5187 \quad$ Alpha for part $2=, 1942$

Reliabilität - Itembatterie "Synergiesystem"

RELIABILITY ANALYSIS- SCALE (SPLIT)

Reliability Coefficients 10 items

Correlation between forms $=, 3400$ Equal-length Spearman-Brown $=, 5075$

Guttman Split-half = ,5055 Unequal-length Spearman-Brown $=, 5075$

Alpha for part $1=, 6451 \quad$ Alpha for part $2=, 6029$

5 items in part $1 \quad 5$ items in part 2

Reliabilität - Itembatterie "Erfolg aus der Beziehungsperspektive“"

RELIABILITYANALYSIS-SCALE (SPLIT)

Reliability Coefficients

$\mathrm{N}$ of Cases $=55,0 \quad \mathrm{~N}$ of Items $=10$

Correlation between forms $=, 2901$ Equal-length Spearman-Brown $=, 44$

Guttman Split-half = ,4497 Unequal-length Spearman-Brown $=, 4497$

5 Items in part $1 \quad 5$ Items in part 2

Alpha for part $1=, 6165 \quad$ Alpha for part $2=, 4266$ 
Reliabilität - Itembatterie "Erfolg aus der Binnenperspektive"

REI I A I L I T Y A A L Y IS- SCALE (SPI I T)

Reliability Coefficients

$\mathrm{N}$ of Cases $=127,0$

$\mathrm{N}$ of Items $=5$

Correlation between forms $=, 3057$

Equal-length Spearman-Brown $=, 4682$

Guttman Split-half $=, 3960$

Unequal-length Spearman-Brown $=, 4749$

3 Items in part 1

2 Items in part 2

Alpha for part $1=, 6699$

Alpha for part $2=, 3854$

Reliabilität - Itembatterie "Erfolg aus der Außenperspektive“

RELIABILITY ANALYSIS- SCALE (SPIIT)

Reliability Coefficients

$\mathrm{N}$ of Cases $=128,0$

$\mathrm{N}$ of Items $=5$

Correlation between forms $=, 3423$ Equal-length Spearman-Brown $=, 5100$

Guttman Split-half $=, 4545$

Unequal-length Spearman-Brown = ,5169

3 Items in part 1

2 Items in part 2

Alpha for part $1=, 6832$

Alpha for part $2=, 3642$

Reliabilität - Itembatterie "Erfolg aus der Finanzperspektive"

RELIABILITY ANALYSIS- SCALE (SPLIT)

Reliability Coefficients

$\mathrm{N}$ of Cases $=129,0$

$\mathrm{N}$ of Items $=3$

Correlation between forms $=, 7828$ Equal-length Spearman-Brown $=, 8782$

Guttman Split-half $=, 8254$

Unequal-length Spearman-Brown $=, 8890$

2 Items in part 1

1 Items in part 2

Alpha for part $1=, 9718$

Alpha for part $2=1,0000$ 

Appendix 4: Konsistenztest - Trennschärfekoeffizienten 
Konsistenztest - Trennschärfekoeffizienten für Itembatterie "Selbstverpflichtung"

\begin{tabular}{|c|c|c|c|c|c|c|c|c|c|c|c|c|}
\hline & & mmen- & erstkoop & früherko & gutkoop & brancgut & infopart & kooprisk & riskok & riskübz & einflerf & immrisk \\
\hline \multirow{4}{*}{$\begin{array}{l}\text { Summen- } \\
\text { score }\end{array}$} & & & & & & & & & & & & \\
\hline & $R$ & 1 &, $572(* *)$ & ,432("*) & $\left..464{ }^{(* *}\right)$ &, $400\left(^{* *}\right)$ &, $\left.501{ }^{(* *}\right)$ & 0,235 & $.462\left({ }^{* *}\right)$ & $.544\left(^{* *}\right)$ & ,331(*) & ,489("*) \\
\hline & Signifikanz (2-seitig) & & & 0,001 & & 0,003 & & 0,084 & & 0 & 0,014 & \\
\hline & & 55 & 55 & 55 & 55 & 55 & 55 & 55 & 55 & 55 & 55 & 55 \\
\hline \multirow[t]{3}{*}{ erstkoop } & $R$ & $.572\left(^{\star \star}\right)$ & 1 &, $287\left(^{*}\right)$ & 0,204 & $-0,052$ & $-0,016$ & $-0,093$ & ,275(") & 0,159 & $-0,23$ & 0,122 \\
\hline & Signifikanz (2-seitig) & & & 0,03 & 0,128 & 0,707 & 0,908 & 0,492 & 0,038 & 0,239 & 0,086 & 0,365 \\
\hline & $\mathrm{N}$ & 55 & 57 & 57 & 57 & 55 & 57 & 57 & 57 & 57 & 57 & 57 \\
\hline \multirow[t]{3}{*}{ früherko } & $R$ & $.432\left(^{* \star}\right)$ & ,287(*) & 1 & 0,204 & $-0,074$ & 0,044 &,$- 432\left({ }^{\star \star}\right)$ & $-0,097$ & $-0,073$ & $-0,027$ & $-0,008$ \\
\hline & Signifikanz (2-seitig) & 0,001 & 0,03 & & 0,127 & 0,593 & 0,744 & 0,001 & 0,472 & 0,591 & 0,842 & 0,954 \\
\hline & & 55 & 57 & 57 & 57 & 55 & 57 & 57 & 57 & 57 & 57 & 57 \\
\hline \multirow[t]{3}{*}{ gutkoop } & $R$ & $.464\left(^{* *}\right)$ & 0,204 & 0,204 & 1 & 0,214 & 0,187 & $-0,083$ & 0,038 & 0,187 & 0,14 & $-0,011$ \\
\hline & Signifikanz (2-seitig) & & 0,128 & 0,127 & & 0,117 & 0,165 & 0,538 & 0,78 & 0,163 & 0,298 & 0,933 \\
\hline & $\mathrm{N}$ & 55 & 57 & 57 & 57 & 55 & 57 & 57 & 57 & 57 & 57 & 57 \\
\hline \multirow[t]{3}{*}{ brancgut } & $R$ &, $400\left(^{* \star}\right)$ & $-0,052$ & $-0,074$ & 0,214 & 1 & $.453\left({ }^{* \star}\right)$ & 0,18 & 0,037 &, $329\left(^{*}\right)$ & 0,223 & 0,085 \\
\hline & Signifikanz (2-seitig) & 0,003 & 0,707 & 0,593 & 0,117 & & 0,001 & 0,19 & 0,787 & 0,014 & 0,101 & 0,538 \\
\hline & & 55 & 55 & 55 & 55 & 55 & 55 & 55 & 55 & 55 & 55 & 55 \\
\hline \multirow[t]{3}{*}{ infopart } & &, $501\left(^{\star \star}\right)$ & $-0,016$ & 0,044 & 0,187 & $.453\left(^{\star \star *}\right)$ & 1 & 0,13 & 0,012 & $340\left(^{\star \star *}\right)$ & 0,172 & 0,204 \\
\hline & Signifikanz (2-seitig) & 0 & 0,908 & 0,744 & 0,165 & 0,001 & & 0,334 & 0,929 & 0,01 & 0,2 & 0,127 \\
\hline & $\mathrm{N}$ & 55 & 57 & 57 & 57 & 55 & 57 & 57 & 57 & 57 & 57 & 57 \\
\hline \multirow[t]{3}{*}{ kooprisk } & $R$ & 0,235 & $-0,093$ &,$- 432\left({ }^{* *}\right)$ & $-0,083$ & 0,18 & 0,13 & 1 & $.403\left({ }^{* *}\right)$ &, $337\left(^{*}\right)$ & 0,206 &, $389\left(^{* \star *}\right)$ \\
\hline & (2-seitig) & 0,084 & 0,492 & 0,001 & 0,538 & 0,19 & 0,334 & & 0,002 & 0,01 & 0,124 & 0,003 \\
\hline & $\mathrm{N}$ & 55 & 57 & 57 & 57 & 55 & 57 & 57 & 57 & 57 & 57 & 57 \\
\hline \multirow[t]{3}{*}{ riskok } & &, $462\left(^{* *}\right)$ & ,275(*) & $-0,097$ & 0,038 & 0,037 & 0,012 & $.403\left(^{* *}\right)$ & 1 & $.450\left(^{\star \star}\right)$ & 0,117 & $.405\left(^{\star *}\right)$ \\
\hline & Signifikanz (2-seitig) & 0 & 0,038 & 0,472 & 0,78 & 0,787 & 0,929 & 0,002 & & 0 & 0,388 & 0,002 \\
\hline & $\mathrm{N}$ & 55 & 57 & 57 & 57 & 55 & 57 & 57 & 57 & 57 & 57 & 57 \\
\hline \multirow[t]{3}{*}{ riskübz } & $\mathrm{R}$ &, $544\left(^{* \star *}\right)$ & 0,159 & $-0,073$ & 0,187 &, $329\left(^{*}\right)$ & $.340(* \star)$ &, $337\left(^{*}\right)$ & $.450\left(^{\star \star}\right)$ & 1 & 0,104 &, $405\left(^{(* *}\right)$ \\
\hline & Signifikanz (2-seitig) & 0 & 0,239 & 0,591 & 0,163 & 0,014 & 0,01 & 0,01 & 0 & & 0,442 & 0,002 \\
\hline & $\mathrm{N}$ & 55 & 57 & 57 & 57 & 55 & 57 & 57 & 57 & 57 & 57 & 57 \\
\hline \multirow[t]{3}{*}{ einflerf } & &, $331\left(^{*}\right)$ & $-0,23$ & $-0,027$ & 0,14 & 0,223 & 0,172 & 0,206 & 0,117 & 0,104 & 1 & 0,233 \\
\hline & Signifikanz (2-seitig) & 0,014 & 0,086 & 0,842 & 0,298 & 0,101 & 0,2 & 0,124 & 0,388 & 0,442 & & 0,082 \\
\hline & $\mathrm{N}$ & 55 & 57 & 57 & 57 & 55 & 57 & 57 & 57 & 57 & 57 & 57 \\
\hline \multirow[t]{3}{*}{ immrisk } & & $.489\left({ }^{\star \star *}\right)$ & 0,122 & $-0,008$ & $-0,011$ & 0,085 & 0,204 &, $\left.3899^{(\star *}\right)$ &, $405\left({ }^{\star \star}\right)$ & $.405\left(^{* *}\right)$ & 0,233 & 3 \\
\hline & Signifikanz (2-seitig) & 0 & 0,365 & 0,954 & 0,933 & 0,538 & 0,127 & 0,003 & 0,002 & 0,002 & 0,082 & \\
\hline & $\mathrm{N}$ & 55 & 57 & 57 & 57 & 55 & 57 & 57 & 57 & 57 & 57 & 57| \\
\hline
\end{tabular}

** Die Korrelation ist auf dem Niveau von 0,01 (2-seitig) signifikant.

* Die Korrelation ist auf dem Niveau von 0,05 (2-seitig) signifikant. 
Konsistenztest - Trennschärfekoeffizlenten für Itembatterie "Synergiesystem"

\begin{tabular}{|c|c|c|c|c|c|c|c|c|c|c|c|c|c|c|}
\hline & & S-score & intensiv & |rechtsel & dbeen & jern & 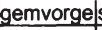 & & & & tents & hego & 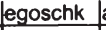 & 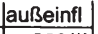 \\
\hline Summen- & & 1 &, $663(* *)$ &, $462\left(^{(* *)}\right.$ &, $425(+*)$ &, $600\left(^{* *}\right)$ & $.666\left(^{*+4}\right)$ & ,391("*) &, $455(+\infty)$ &, $703\left({ }^{(+4)}\right.$ &, $622(+*)$ & $\left., 454)^{* *}\right)$ &, $449\left(^{(*)}\right.$ &, $283\left({ }^{*}\right)$ \\
\hline \multirow[t]{2}{*}{ score } & Signifikanz (2-seitig) & & & & 0,001 & & & 0,003 & & & & & & 0,033 \\
\hline & $\mathrm{N}$ & 57 & 57 & 57) & 57 & 57 & 57 & 57 & 57 & 57 & 57 & 57 & 57 & 57 \\
\hline \multirow[t]{3}{*}{ intensiv } & $\mathbf{R}$ & ,663(") & 1 &, $405(" \omega)$ & 0,234 &, 419()$\left.^{*+1}\right)$ & ,309(*) & 0,193 & 0,12 & ,597("*) &, $395(+4)$ & 0,146 & 0,153 & ,286(*) \\
\hline & Signifikanz (2-seitig) & & & 0,002 & 0,08 & 0,001 & 0,019 & 0,15 & 0,375 & 0 & 0,002 & 0,28 & 0,256 & 0,031 \\
\hline & & 57 & 57 & 57 & 57 & 57 & 57 & 57 & 57 & 57 & 57 & 57 & 57 & 57 \\
\hline \multirow[t]{3}{*}{ rechtsel } & $\mathbf{R}$ &, $462(*)$ & ,405("+) & 1 & ,380("*) & 0,046 & 0,13 & 0,152 & $-0,082$ & ,313(") & ,267(") & 0,138 & $-0,038$ & 0,144 \\
\hline & Signifikanz (2-seitig) & & 0,002 & & 0,004 & 0,735 & 0,335 & 0,26 & 0,542 & 0,018 & 0,045 & 0,308 & 0,782 & 0,287 \\
\hline & & 57 & 57 & 57 & 57 & 57 & 57 & 57 & 57 & 57 & 57 & 57. & 57 & 57 \\
\hline \multirow[t]{3}{*}{ jedbeend } & $R$ &, $425\left({ }^{*++}\right)$ & 0,234 &, $380\left(^{+*}\right)$ & 1 & 0,017 & 0,134 & $-0,06$ & 0,131 & 0,22 & ,297( $\left(^{*}\right)$ & 0,018 & 0,073 & ,267(") \\
\hline & Signifikanz (2-seitig) & 0,001 & 0,08 & 0,004 & & 0,901 & 319 & 655 & 0,33 & 0,1 & 0,025 & 892 & 589 & 0,045 \\
\hline & & 57 & 57 & 57 & 57 & 57 & 57 & 57 & 57 & 57 & 57 & 57 & 57 & 57 \\
\hline \multirow[t]{3}{*}{ abstverh } & &, $600\left(^{*+*}\right)$ & ,419("*) & 0,046 & 0,017 & 1 &, $640\left(^{(-\infty}\right)$ & 0,127 & 119 &, $580(" *)$ & $.420(" \times)$ &, 064 & 0,045 & 0,105 \\
\hline & Signifikanz (2-seitig) & 0 & 0,001 & 0,735 & 0,901 & & 0 & 0,346 & 0,378 & 0 & 0,001 & 634 & 0,742 & 0,437 \\
\hline & $\mathrm{N}$ & 57 & 57 & 57 & 57 & 57 & 57 & 57 & 57 & 57 & 57 & 57 & 57 & 57 \\
\hline \multirow[t]{3}{*}{ gemvorge } & &, $666\left(^{* *}\right)$ & ,309(") & 0,13 & 0,134 & ,640("+*) & 1 & ,286(") & ,414(**) &, $\left.3761^{* *}\right)$ &, $320(*)$ & 0,129 & 0,238 & 0,215 \\
\hline & Signifikanz (2-seitig) & & 0,019 & 0,335 & 0,319 & 0 & & 0,031 & 0,001 & 0.004 & 0,015 & 0,339 & 0,074 & 0,108 \\
\hline & $\mathrm{N}$ & 57 & 57 & 57 & 57 & 57 & 57 & 57 & 57 & 57 & 57 & 57 & 57 & 57 \\
\hline \multirow[t]{3}{*}{ sprekoop } & & ,391("*) & 0,193 & 0,152 & $-0,06$ & 0,127 & ,286(") & 1 & 0,095 & ,271(*) & 0,092 & 0,192 & 0,131 & 0,044 \\
\hline & Signifikanz (2-seitig) & 0,003 & 0,15 & 0,26 & 0,655 & 0,346 & 0,031 & & 0,481 & 0,042 & 0,496 & 0,152 & 0,33 & 0,744 \\
\hline & $\mathrm{N}$ & 57 & 57 & 57 & 57 & 57 & 57 & 57 & 57 & 57 & 57 & 57 & 57 & 57 \\
\hline \multirow[t]{3}{*}{ schnerfo } & $\mathbf{R}$ &, $455(")$ & 0,12 & $-0,082$ & 0,131 & 0,119 &, $414($ (") & 0,095 & 1 & 0,112 & ,266(") &, $326\left(^{*}\right)$ & ,322(") &, $322\left(^{*}\right)$ \\
\hline & Signifikanz (2-seitig) & 0 & 0,375 & 0,542 & 0,33 & 0,378 & 0,001 & 0,481 & & 0,405 & 0,046 & 0,013 & 0,015 & 0,015 \\
\hline & $\mathrm{N}$ & 57 & 57 & 57 & 57 & 57 & 57 & 57 & 57 & 57 & 57 & 57 & 57 & 57 \\
\hline \multirow[t]{3}{*}{ gleiinte } & $\mathbf{R}$ &, $703\left(^{* *}\right)$ &, $597(* *)$ &, $313\left(^{*}\right)$ & 0,22 &, $580\left(^{(* t)}\right.$ &, $376\left(\left(^{*-4}\right)\right.$ &, $271\left(^{*}\right)$ &, 112 & 1 & $.525\left(^{*+t}\right)$ & 0,133 & 0,162 & ,286(") \\
\hline & Signifikanz (2-seitig) & & & 0,018 & 0,1 & & 0,004 & 0,042 & 0,405 & & & 0,324 & 0,227 & 0,031 \\
\hline & $\mathrm{N}$ & 57 & 57 & 57 & 57 & 57 & 57 & 57 & 57 & 57 & 57 & 57 & 57 & 57 \\
\hline \multirow[t]{3}{*}{ entschgl } & $\mathbf{R}$ &, $622(* *)$ & ,395(*) & ,267(") & ,297(") &, $420\left(^{* *+}\right)$ &, $320\left({ }^{*}\right)$ & 0,092 & ,266(") &, $525(*)$ & 1 & 0,156 & 0,23 &, $555\left({ }^{(* *}\right)$ \\
\hline & Signifikanz (2-seitig) & & 0,002 & 0,045 & 0,025 & 0,001 & 0,015 & 0,496 & 0,046 & 0 & & 0,245 & 0,085 & \\
\hline & $N$ & 57 & 57 & 57 & 57 & 57 & 57 & 57 & 57 & 57 & 57 & 57 & 57 & 57 \\
\hline \multirow[t]{3}{*}{ ichego } & $R$ &, $454\left(^{+*}\right)$ & 0,146 & 0,138 & 0,018 & 0,064 & 0,129 & 0,192 & ,326(") & 0,133 & 0,156 & 1 &, $503\left(^{* *}\right)$ & 0,152 \\
\hline & Signifikanz (2-seitig) & 0 & 0,28 & 0,308 & 0,892 & 0,634 & 0,339 & 0,152 & 0,013 & 0,324 & 0,245 & & 0 & 0,258 \\
\hline & $\mathrm{N}$ & 57 & 57 & 57 & 57 & 57 & 57 & 57 & 57 & 57 & 57 & 57 & 57 & 57 \\
\hline \multirow[t]{3}{*}{ egoschk } & &, $449\left(^{+*+}\right)$ & 0,153 & $-0,038$ & 0,073 & 0,045 & 0,238 & 0,131 & ,322(*) & 0,162 & 0,23 & $.503\left(^{* * *}\right)$ & 1 & 0,223 \\
\hline & Signifikanz (2-seitig) & 0 & 0,256 & 0,782 & 0,589 & 0,742 & 0,074 & 0,33 & 0,015 & 0,227 & 0,085 & 0 & & 0,095 \\
\hline & $\mathrm{N}$ & 57 & 57 & 57 & 57 & 57 & 57 & 57 & 57 & 57 & 57 & 57 & 57 & 57 \\
\hline \multirow[t]{3}{*}{ außeinfl } & & ,283(") & ,286(") & 0,144 & ,267(*) & 0,105 & 0,215 & 0,044 &, $322(*)$ & ,286(") &, $555\left({ }^{(*)}\right)$ & 0,152 & 0,223 & 1 \\
\hline & Signifikanz (2-seitig) & 0,033 & 0,031 & 0,287 & 0,045 & 0,437 & 0,108 & 0,744 & 0,015 & 0,031 & 0 & 0,258 & 0,095 & \\
\hline & & 57 & 57 & 57 & 57 & 57 & 57 & 57 & 57 & 57 & 57 & 57 & 57 & 57 \\
\hline
\end{tabular}


Konsistenztest - Trennschärfekoeffizienten für Itembatterie "Erfolg aus der Beziehungsperspektive"

\begin{tabular}{|c|c|c|c|c|c|c|c|c|c|c|c|c|}
\hline & & & & & & & & & & & & \\
\hline $\begin{array}{l}\text { Summe } \\
\text { score }\end{array}$ & $\begin{array}{r}\mathbf{R} \\
\text { Signifikanz (2-seitig) } \\
\mathrm{N}\end{array}$ & 56 & $\begin{array}{r}.541(+4) \\
0 \\
56 \\
\end{array}$ & $\begin{array}{r}, 284(*) \\
0,036 \\
55 \\
\end{array}$ & $\begin{array}{r}.770(+4) \\
0 \\
56 \\
\end{array}$ & $\begin{array}{r}.617(+*+1) \\
0 \\
56 \\
\end{array}$ & $\begin{array}{r}.636(+4) \\
0 \\
56 \\
\end{array}$ & $\begin{array}{r}, 431(* m) \\
0,001 \\
56 \\
\end{array}$ & $\begin{array}{r}, 384(+4) \\
0,004 \\
56 \\
\end{array}$ & $\begin{array}{r}, 568(+4) \\
0 \\
56 \\
\end{array}$ & $\begin{array}{r}.523(+*) \\
0 \\
56 \\
\end{array}$ & $\begin{array}{r}0,139 \\
0,308 \\
56 \\
\end{array}$ \\
\hline erreichb & $\begin{array}{r}\mathbf{R} \\
\text { Signifikanz (2-seitig) } \\
\mathrm{N} \\
\end{array}$ & $\begin{array}{r}541(+14) \\
0 \\
56 \\
\end{array}$ & 57 & $\begin{array}{r}0,042 \\
0,761 \\
56 \\
\end{array}$ & $\begin{array}{r}341\left(^{++\infty}\right. \\
0,01 \\
57 \\
\end{array}$ & $\begin{array}{r}321\left(^{*}\right) \\
0,015 \\
57 \\
\end{array}$ & $\begin{array}{r}296\left(^{*}\right) \\
0,025 \\
57 \\
\end{array}$ & $\begin{array}{r}, 263(*) \\
0,048 \\
57 \\
\end{array}$ & $\begin{array}{r}0,134 \\
0,322 \\
57 \\
\end{array}$ & $\begin{array}{r}, 378(+\infty) \\
0,004 \\
57 \\
\end{array}$ & $\begin{array}{r}0,213 \\
0,112 \\
57 \\
\end{array}$ & $\begin{array}{r}-0,14 \\
0,303 \\
56 \\
\end{array}$ \\
\hline taktik & $\begin{array}{r}\mathbf{R} \\
\text { Signifikanz (2-seitig) } \\
\mathrm{N} \\
\end{array}$ & $\begin{array}{r}284(*) \\
0,036 \\
55 \\
\end{array}$ & $\begin{array}{r}0,042 \\
0,761 \\
56 \\
\end{array}$ & 56 & $\begin{array}{r}0,079 \\
0,563 \\
56 \\
\end{array}$ & $\begin{array}{r}.559(+4) \\
0 \\
56 \\
\end{array}$ & $\begin{array}{r}0,189 \\
0,162 \\
56 \\
\end{array}$ & $\begin{array}{r}0,144 \\
0,289 \\
56 \\
\end{array}$ & $\begin{array}{r}-0,036 \\
0,791 \\
56 \\
\end{array}$ & $\begin{array}{r}-0,094 \\
0,49 \\
56 \\
\end{array}$ & $\begin{array}{r}, 299(*) \\
0,025 \\
56 \\
\end{array}$ & $\begin{array}{r}-0,016 \\
0,909 \\
55 \\
\end{array}$ \\
\hline sorprobl & $\begin{array}{r}\mathbf{R} \\
\text { Signifikanz (2-seitig) } \\
\mathrm{N} \\
\end{array}$ & $\begin{array}{r}.770(* 4) \\
0 \\
56 \\
\end{array}$ & $\begin{array}{r}, 341(*+1) \\
0,01 \\
57 \\
\end{array}$ & $\begin{array}{r}0,079 \\
0,563 \\
56 \\
\end{array}$ & 57 & $\begin{array}{r}, 398(*+1) \\
0,002 \\
57 \\
\end{array}$ & $\begin{array}{r}.588(+4) \\
0 \\
57 \\
\end{array}$ & $\begin{array}{r}, 441(+*) \\
0,001 \\
57 \\
\end{array}$ & $\begin{array}{r}0,127 \\
0,345 \\
57 \\
\end{array}$ & \begin{tabular}{|r|}
$.402(* \pi)$ \\
0,002 \\
57 \\
\end{tabular} & $\begin{array}{r}, 379(+*) \\
0,004 \\
57 \\
\end{array}$ & $\begin{array}{r}-0,021 \\
0,877 \\
56 \\
\end{array}$ \\
\hline plmens & $\begin{array}{r}\mathbf{R} \\
\text { Signifikanz (2-seitig) } \\
\mathrm{N} \\
\end{array}$ & $\begin{array}{r}, 617(+4) \\
0 \\
56 \\
\end{array}$ & $\begin{array}{r}, 321(*) \\
0,015 \\
57 \\
\end{array}$ & $\begin{array}{r}, 559(+\pi) \\
0 \\
56 \\
\end{array}$ & $\begin{array}{r}, 398(*+4) \\
0,002 \\
57 \\
\end{array}$ & 57 & $\begin{array}{r}, 456(+*) \\
0 \\
57 \\
\end{array}$ & $\begin{array}{r}0,201 \\
0,134 \\
57 \\
\end{array}$ & $\begin{array}{r}-0,048 \\
0,72 \\
57 \\
\end{array}$ & $\begin{array}{r}0,103 \\
0,444 \\
57 \\
\end{array}$ & $\begin{array}{r}, 328\left({ }^{*}\right) \\
0,013 \\
57 \\
\end{array}$ & $\begin{array}{r}-0,067 \\
0,624 \\
56 \\
\end{array}$ \\
\hline sph & $\begin{array}{r}\mathbf{R} \\
\text { Signifikanz (2-seitig) } \\
\mathbf{N} \\
\end{array}$ & $\begin{array}{r}.636(+*) \\
0 \\
56 \\
\end{array}$ & $\begin{array}{r}.296\left(^{*}\right. \\
0,025 \\
57 \\
\end{array}$ & $\begin{array}{r}\mathbf{0 , 1 8 9} \\
0,162 \\
56 \\
\end{array}$ & $\begin{array}{r}.588(+\infty) \\
0 \\
57 \\
\end{array}$ & $\begin{array}{r}.456(+*) \\
0 \\
57 \\
\end{array}$ & 57 & $\begin{array}{r}, 480(+\infty) \\
0 \\
57 \\
\end{array}$ & $\begin{array}{r}-0,022 \\
0,87 \\
57 \\
\end{array}$ & $\begin{array}{r}0,13 \\
0,337 \\
57 \\
\end{array}$ & $\begin{array}{r}, 281(*) \\
0,034 \\
57 \\
\end{array}$ & $\begin{array}{r}-0,151 \\
0,266 \\
56 \\
\end{array}$ \\
\hline diskerg & $\begin{array}{r}\mathbf{R} \\
\text { Signifikanz (2-seitig) } \\
\mathrm{N} \\
\end{array}$ & $\begin{array}{r}, 431(* * 1) \\
0,001 \\
56 \\
\end{array}$ & $\begin{array}{r}263\left({ }^{*}\right) \\
0,048 \\
57 \\
\end{array}$ & $\begin{array}{r}0,144 \\
0,289 \\
56 \\
\end{array}$ & $\begin{array}{r}441(+4) \\
0,001 \\
57 \\
\end{array}$ & $\begin{array}{r}0,201 \\
0,134 \\
57 \\
\end{array}$ & $\begin{array}{r}180(+\infty) \\
0 \\
57 \\
\end{array}$ & 57 & $\begin{array}{r}, 087 \\
, 518 \\
57 \\
\end{array}$ & $\begin{array}{r}0,015 \\
0,912 \\
57 \\
\end{array}$ & $\begin{array}{r}0,088 \\
0,514 \\
57 \\
\end{array}$ & $\begin{array}{r}399\left({ }^{+4}\right) \\
0,002 \\
56 \\
\end{array}$ \\
\hline ivkont & $\begin{array}{r}\mathbf{R} \\
\text { Signifikanz (2-seitig) } \\
\mathrm{N} \\
\end{array}$ & $\begin{array}{r}384(* 4) \\
0,004 \\
56 \\
\end{array}$ & $\begin{array}{r}0,134 \\
0,322 \\
57 \\
\end{array}$ & $\begin{array}{r}-0,036 \\
0,791 \\
56 \\
\end{array}$ & $\begin{array}{r}0,127 \\
0,345 \\
57 \\
\end{array}$ & $\begin{array}{r}-0,048 \\
0,72 \\
57 \\
\end{array}$ & $\begin{array}{r}-0,022 \\
0,87 \\
57 \\
\end{array}$ & $\begin{array}{r}0,087 \\
0,518 \\
57 \\
\end{array}$ & & $\begin{array}{r}338\left(^{*}\right) \\
0,01 \\
57 \\
\end{array}$ & $\begin{array}{r}0,034 \\
0,799 \\
57 \\
\end{array}$ & $\begin{array}{r}-0,05 \\
0,712 \\
56 \\
\end{array}$ \\
\hline olkenne & Signifikanz (2-seitig) & $\begin{array}{r}, 568(+4) \\
0 \\
56 \\
\end{array}$ & $\begin{array}{r}, 378(+*) \\
0,004 \\
57 \\
\end{array}$ & $\begin{array}{r}-0,094 \\
0,49 \\
56 \\
\end{array}$ & $\begin{array}{r}, 402(+\cdots) \\
0,002 \\
57 \\
\end{array}$ & $\begin{array}{r}0,103 \\
0,444 \\
57 \\
\end{array}$ & $\begin{array}{r}0,13 \\
0,337 \\
57 \\
\end{array}$ & $\begin{array}{r}\mathbf{0 , 0 1 5} \\
0,912 \\
57 \\
\end{array}$ & $\begin{array}{r}, 338(*) \\
0,01 \\
57 \\
\end{array}$ & & $\begin{array}{r}, 296\left({ }^{*}\right) \\
0,026 \\
57 \\
\end{array}$ & $\begin{array}{r}0,101 \\
0,461 \\
56 \\
\end{array}$ \\
\hline achfrag & $\begin{array}{r}\mathbf{R} \\
\text { Signifikanz (2-seitig) } \\
\mathrm{N} \\
\end{array}$ & $\begin{array}{r}, 523(+\pi) \\
0 \\
56 \\
\end{array}$ & $\begin{array}{r}\mathbf{0 , 2 1 3} \\
0,112 \\
57 \\
\end{array}$ & $\begin{array}{r}, 299(*) \\
0,025 \\
56 \\
\end{array}$ & $\begin{array}{r}, 379(+\infty 1) \\
0,004 \\
57 \\
\end{array}$ & $\begin{array}{r}.328(*) \\
0,013 \\
57 \\
\end{array}$ & $\begin{array}{r}, 281(*) \\
0,034 \\
57 \\
\end{array}$ & $\begin{array}{r}0,088 \\
0,514 \\
57 \\
\end{array}$ & $\begin{array}{r}0,034 \\
0,799 \\
57 \\
\end{array}$ & $\begin{array}{r}.296\left(^{*}\right) \\
0,026 \\
57 \\
\end{array}$ & 57 & $\begin{array}{r}-0,048 \\
0,728 \\
56 \\
\end{array}$ \\
\hline 然 & Signifikanz (2-seitig) & $\begin{array}{r}0,139 \\
0,308 \\
56\end{array}$ & $\begin{array}{r}-0,14 \\
0,303 \\
56\end{array}$ & $\begin{array}{r}-0,016 \\
0,909 \\
55\end{array}$ & $\begin{array}{r}-0,021 \\
0,877 \\
56\end{array}$ & $\begin{array}{r}-0,067 \\
0,624 \\
56\end{array}$ & $\begin{array}{r}-0,151 \\
0,266 \\
56\end{array}$ & $\begin{array}{r}-, 399(\#) \\
0,002 \\
56\end{array}$ & $\begin{array}{r}-0,05 \\
0,712 \\
56\end{array}$ & $\begin{array}{r}0,101 \\
0,461 \\
56\end{array}$ & $\begin{array}{r}-0,048 \\
0,728 \\
56\end{array}$ & $\begin{array}{l}8 \\
8 \\
6\end{array}$ \\
\hline
\end{tabular}




\begin{tabular}{|c|c|c|c|c|c|c|c|}
\hline & & $\begin{array}{l}\text { Summen- } \\
\text { score }\end{array}$ & kopmitar & mitqualk & prognose & mitgua3 & mermit3 \\
\hline \multirow{3}{*}{$\begin{array}{l}\text { Summen- } \\
\text { score }\end{array}$} & $\mathbf{R}$ & 1 &, $260(+*)$ & $-0,067$ &, $528(* *)$ &, $477(+\cdots)$ &, $578(+\infty)$ \\
\hline & Signifikanz (2-seitig) & & 0,003 & 0,455 & 0 & & 0 \\
\hline & $\mathrm{N}$ & 127 & 127 & 127 & 127 & 127 & 127 \\
\hline \multirow[t]{3}{*}{ kopmitar } & $\mathbf{R}$ &, $260(+4)$ & 1 &, $773\left(^{(m)}\right.$ & ,249("\#) & 0,12 & ,201(") \\
\hline & Signifikanz (2-seitig) & 0,003 & & 0 & 0,005 & 0,175 & 0,022 \\
\hline & $\mathrm{N}$ & 127 & 129 & 129 & 127 & 129 & 129 \\
\hline \multirow[t]{3}{*}{ mitqualk } & $\mathbf{R}$ & $-0,067$ &, $773\left(^{* *}\right)$ & 1 & ,227(") & 0,133 & 0,099 \\
\hline & Signifikanz (2-seitig) & 0,455 & 0 & & 0,01 & 0,133 & 0,26 \\
\hline & $\mathrm{N}$ & 127 & 129 & 130 & 128 & 130 & 130 \\
\hline \multirow[t]{3}{*}{ prognose } & $\mathbf{R}$ &, $528(*)$ &, $249\left(^{(+*)}\right.$ & $.227\left(^{*}\right)$ & 1 &, $398\left(^{(-4}\right)$ &, $327(+)$ \\
\hline & Signifikanz (2-seitig) & & 0,005 & 0,01 & & 0 & 0 \\
\hline & $\mathrm{N}$ & 127 & 127 & 128 & 128 & 128 & 128 \\
\hline \multirow[t]{3}{*}{ mitqua3 } & $\mathbf{R}$ &, $477\left(^{(-*)}\right.$ & 0,12 & 0,133 & ,398("**) & 1 &, $220(")$ \\
\hline & Signifikanz (2-seitig) & & 0,175 & 0,133 & 0 & & 0,012 \\
\hline & $\mathrm{N}$ & 127 & 129 & 130 & 128 & 130 & 130 \\
\hline \multirow[t]{3}{*}{ mermit3 } & &, $578\left(^{+\infty}\right)$ & ,201(") & 0,099 &, $327(*)$ & $.220\left(^{*}\right)$ & 1 \\
\hline & Signifikanz (2-seitig) & & 0,022 & 0,26 & & 0,012 & \\
\hline & & 127 & 129 & 130 & 128 & 130 & 130 \\
\hline
\end{tabular}

* Die Korrelation ist auf dem Niveau von 0,01 (2-seitig) signifikant.

- Die Korrelation ist auf dem Niveau von 0,05 (2-seitig) signifikant. 
Konsistenztest - Trennschärfekoeffizienten für Itembatterie "Erfolg aus der Außenperspektive"

\begin{tabular}{|c|c|c|c|c|c|c|c|c|}
\hline & & $\begin{array}{l}\text { Summen- } \\
\text { score }\end{array}$ & kundzufr & stamkund & stamlief & angebzus & markplu3 & mantiplu \\
\hline \multirow{3}{*}{$\begin{array}{l}\text { Summen- } \\
\text { scores }\end{array}$} & & 1 &, $545(" \#)$ &, $726\left(^{(\#)}\right.$ &, $782(* \star)$ &, $340\left(^{(* *}\right)$ &, $647(* *)$ &, $591\left({ }^{* *}\right)$ \\
\hline & Signifikanz (2-seitig) & & & & & 0 & 0 & \\
\hline & $\mathrm{N}$ & 128 & 128 & 128 & 128 & 128 & 128 & 128 \\
\hline \multirow[t]{3}{*}{ kundzufr } & &, $545\left(^{(*)}\right.$ & 1 &, $269(*)$ &, $395(* *)$ & $-0,019$ &, $217\left(^{*}\right)$ & 0,091 \\
\hline & Signifikanz (2-seitig) & & & 0,002 & & 0,833 & 0,014 & 0,307 \\
\hline & $\mathrm{N}$ & 128 & 129 & 129 & 129 & 129 & 129 & 128 \\
\hline \multirow[t]{3}{*}{ stamkund } & &, $726(* \#)$ &, $269(* *)$ & 1 &, $573(* *)$ &, $190\left(^{*}\right)$ &, $215\left(^{*}\right)$ &, $311(* \pi)$ \\
\hline & Signifikanz (2-seitig) & & 0,002 & & & 0,031 & 0,014 & \\
\hline & $\mathrm{N}$ & 128 & 129 & 130 & 130 & 130 & 130 & 129 \\
\hline \multirow[t]{3}{*}{ stamlief } & & ,782(**) &, $395\left(^{(*)}\right.$ &, $573\left(^{(k)}\right.$ & 1 &, $255\left(^{\star \star}\right)$ &, $377\left(^{(* t)}\right)$ & ,220(*) \\
\hline & Signifikanz (2-seitig) & & & 0 & & 0,003 & 0 & 0,012 \\
\hline & $\mathrm{N}$ & 128 & 129 & 130 & 130 & 130 & 130 & 129 \\
\hline \multirow[t]{3}{*}{ angebzus } & &, $340\left(^{(*-\pi}\right)$ & $-0,019$ & ,190(*) &, $255\left({ }^{\star \star *}\right)$ & 1 &, $375\left({ }^{* *}\right)$ &, $324(* \pi)$ \\
\hline & Signifikanz (2-seitig) & & 0,833 & 0,031 & 0,003 & & 0 & \\
\hline & $\mathrm{N}$ & 128 & 129 & 130 & 130 & 130 & 130 & 129 \\
\hline \multirow[t]{3}{*}{ markplu3 } & &, $647\left(^{(\star *}\right)$ &, $217\left(^{*}\right)$ & ,215(*) &, $377(* 4)$ &, $375(* t)$ & 1 &, $501\left(^{* *}\right)$ \\
\hline & Signifikanz (2-seitig) & & 0,014 & 0,014 & 0 & 0 & & \\
\hline & $\mathrm{N}$ & 128 & 129 & 130 & 130 & 130 & 130 & 129 \\
\hline \multirow[t]{3}{*}{ mantlplu } & &, $591\left(^{(\star *}\right)$ & 0,091 &, $311\left(^{(* t}\right)$ &, $220\left(^{*}\right)$ &, $324\left({ }^{* *}\right)$ &, $501\left(^{* \star}\right)$ & 1 \\
\hline & Signifikanz (2-seitig) & & 0,307 & & 0,012 & & 0 & \\
\hline & $N$ & 128 & 128 & 129 & 129 & 129 & 129 & 129 \\
\hline
\end{tabular}

** Die Korrelation ist auf dem Niveau von 0,01 (2-seitig) signifikant.

* Die Korrelation ist auf dem Niveau von 0,05 (2-seitig) signifikant. 
Konsistenztest - Trennschärfekoeffizienten für Itembatterie "Erfolg aus der Finanzperspektive"

\begin{tabular}{|c|c|c|c|c|c|c|c|c|}
\hline & & $\begin{array}{l}\text { Summen- } \\
\text { score }\end{array}$ & cfplu3 & umsplu3 & merinv3 & umsplus & cfplus & mehrinv \\
\hline \multirow{3}{*}{$\begin{array}{l}\text { Summen- } \\
\text { scores }\end{array}$} & & 1 &, $675\left(^{(m)}\right.$ &, $769\left({ }^{* *}\right)$ & ,655( &, $515\left(^{(-4}\right)$ &, $511(*-)$ &, $511(*)$ \\
\hline & Signifikanz (2-seitig) & & & & & 0 & 0 & 0 \\
\hline & & 129 & 129 & 129 & 129 & 129 & 129 & 129 \\
\hline \multirow[t]{3}{*}{ cfplu3 } & $\mathbf{R}$ &, $675\left(^{(+*)}\right.$ & 1 &, $814\left(^{* *}\right)$ &, $252\left({ }^{* *}\right)$ &, $313\left(^{* *}\right)$ &, $353(*)$ &, $315\left(^{* *}\right)$ \\
\hline & Signifikanz (2-seitig) & & & & 0,004 & & & \\
\hline & $\mathrm{N}$ & 129 & 129 & 129 & 129 & 129 & 129 & 129 \\
\hline \multirow[t]{3}{*}{ umsplu3 } & $R$ & ,769("*) & $814($ (\#) & 1 &, $334\left(^{*+\infty}\right)$ & ,431(*) & ,433("- &, $413(-\infty)$ \\
\hline & Signifikanz (2-seitig) & & & & & & 0 & \\
\hline & & 129 & 129 & 130 & 130 & 130 & 130 & 130 \\
\hline \multirow[t]{3}{*}{ merinv3 } & &, $655(*)$ & ,252("*it) &, $334\left(^{* *}\right)$ & 1 &, $436\left(^{-m}\right)$ & ,422("-i) &, $461(*)$ \\
\hline & Signifikanz (2-seitig) & & 0,004 & 0 & & 0 & 0 & \\
\hline & & 129 & 129 & 130 & 130 & 130 & 130 & 130 \\
\hline \multirow[t]{3}{*}{ umsplus } & &, $515\left(^{(-\star}\right)$ &, $313(* *)$ &, $431\left(^{+\infty}\right)$ &, $436\left(^{(-4)}\right.$ & 1 &, $978(-*)$ & ,951(**) \\
\hline & Signifikanz (2-seitig) & & & 0 & 0 & & 0 & 0 \\
\hline & & 129 & 129 & 130 & 130 & 130 & 130 & 130 \\
\hline \multirow[t]{3}{*}{ cfplus } & &, $511\left(^{+\infty}\right)$ &, $353\left(^{* \star *}\right)$ &, $433\left(^{+*}\right)$ & ,422( $\left(^{*+4}\right)$ &, $978\left(^{* *}\right)$ & 1 &, $953\left({ }^{*+*}\right)$ \\
\hline & Signifikanz (2-seitig) & & & 0 & 0 & 0 & & 0 \\
\hline & 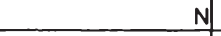 & 129 & 129 & 130 & 130 & 130 & 130 & 130 \\
\hline \multirow[t]{3}{*}{ mehrinv } & &, $511(+*)$ &, $315\left(^{* *}\right)$ &, $413(-4)$ &, $461(+\infty)$ &, $951\left(^{(+4}\right)$ &, $953(++)$ & 1 \\
\hline & Signifikanz (2-seitig) & & & & & & 0 & \\
\hline & & 129 & 129 & 130 & 130 & 130 & 130 & 130| \\
\hline
\end{tabular}

** Die Korrelation ist auf dem Niveau von 0,01 (2-seitig) signifikant.

- Die Korrelation ist auf dem Niveau von 0,05 (2-seitig) signifikant. 

Appendix 5: Formeln aus SPSS 10 


\section{Grad der Selbstverpflichtung:}

IF $($ kooperat $=1)$ selbstpf $=$ erstkoop + früherko + gutkoop + brancgut + infopart + kooprisk + riskok + einflerf + riskübz + immrisk .

EXECUTE .

\section{Grad der Synergiesystemausprägungen:}

IF $($ kooperat $=1)$ synerg $=$ intensiv + rechtsel + jedbeend + abstverh + gemvorge + sprekoop + schnerfo + gleiinte + entschgl + ichego + egoschk - außeinfl .

EXECUTE .

Erfolg aus der Beziehungsperspektive:

IF $($ kooperat $=1)$ gbezerf2 $=$ erreichb + anspherz + sprprobl + diskerg + privkont + koplmens + ablkenne + nachfrag .

EXECUTE .

\section{Erfolg aus der Binnenperspektive:}

IF (kooperat $=1)$ gbinerfk $=$ kopmitar + mitqualk + prognose .

EXECUTE .

IF $($ kooperat $=0)$ gbinerfn $=$ mitqua $3+$ mermit $3+$ prognose.

EXECUTE .

COMPUTE gbinerf $=$ gbinerfk + gbinerfn .

EXECUTE .

\section{Erfolg aus der Außenperspektive}

IF $($ kooperat $=1)$ gauserfk $=$ kundzufr + stamkund + stamlief + mantlplu .

EXECUTE .

IF $($ kooperat $=0)$ gauserfn $=$ kundzufr + stamkund + stamlief + markplu3.

EXECUTE .

COMPUTE gaußerf $=$ gauserfk + gauserfn .

EXECUTE .

\section{Erfolg aus der Finanzperspektive}

IF $($ kooperat $=1)$ gfinerfk $=$ umsplus + cfplus + mehrinv .

EXECUTE .

IF $($ kooperat $=0)$ gfinerfn $=$ umsplu3 + cfplu3 + merinv3.

EXECUTE .

COMPUTE gfinerf $=$ gfinerfk + gfinerfn.

EXECUTE . 
Appendix 6: Korrelationsmatrizen 
Korrelationsmatrix - Modelitest/Hypothesentest

\begin{tabular}{|c|c|c|c|c|c|c|c|}
\hline & & $\begin{array}{l}\text { Grad der } \\
\text { Selbstverpflichtung }\end{array}$ & $\begin{array}{l}\text { Grad der } \\
\text { Synergiesys- } \\
\text { temauspräg- } \\
\text { ungen }\end{array}$ & $\begin{array}{l}\text { Erfolg aus der } \\
\text { Beziehungs- } \\
\text { perspektive }\end{array}$ & $\begin{array}{l}\text { Erfolg aus } \\
\text { der Binnen- } \\
\text { perspektive }\end{array}$ & $\begin{array}{l}\text { Erfolg aus } \\
\text { der Außen- } \\
\text { perspektive }\end{array}$ & $\begin{array}{l}\text { Erfolg aus } \\
\text { der Finanz- } \\
\text { perspektive }\end{array}$ \\
\hline & $\begin{array}{r}\mathbf{R} \\
\text { Signifikanz }(2- \\
\text { seitig) } \\
\mathrm{N} \\
\end{array}$ & $\begin{array}{r}\prime \\
55 \\
\end{array}$ & $\begin{array}{r}, 519(*-) \\
0 \\
55 \\
\end{array}$ & $\begin{array}{r}0,256 \\
0,061 \\
54 \\
\end{array}$ & $\begin{array}{r}0,072 \\
0,605 \\
54 \\
\end{array}$ & $\begin{array}{r}0,047 \\
0,731 \\
55 \\
\end{array}$ & $\begin{array}{r}0,232 \\
0,088 \\
55 \\
\end{array}$ \\
\hline $\begin{array}{l}\text { Grad der } \\
\text { Synergiesystem- } \\
\text { ausprägungen }\end{array}$ & $\begin{array}{r}\mathbf{R} \\
\text { Signifikanz (2- } \\
\text { seitig) } \\
\mathrm{N} \\
\end{array}$ & $\begin{array}{r}, 519(*) \\
0 \\
55 \\
\end{array}$ & 57 & $\begin{array}{r}.498(* *) \\
0 \\
56 \\
\end{array}$ & $\begin{array}{r}.415\left(^{\star-}\right) \\
0,002 \\
55 \\
\end{array}$ & $\begin{array}{r}.437(\text { (t) } \\
0,001 \\
56 \\
\end{array}$ & $\begin{array}{r}, 470\left(^{* *}\right) \\
0 \\
57 \\
\end{array}$ \\
\hline $\begin{array}{l}\text { Erfolg aus der } \\
\text { Beziehungs- } \\
\text { perspektive }\end{array}$ & $\begin{array}{r}\mathbf{R} \\
\text { Signifikanz (2- } \\
\text { seitig) } \\
\mathrm{N} \\
\end{array}$ & $\begin{array}{r}0,256 \\
0,061 \\
54 \\
\end{array}$ & $\begin{array}{r}, 498(\star \star) \\
0 \\
56 \\
\end{array}$ & 56 & $\begin{array}{r}0,158 \\
0,255 \\
54 \\
\end{array}$ & $\begin{array}{r}, 345\left(^{\star \star}\right) \\
0,01 \\
55 \\
\end{array}$ & $\begin{array}{r}0,034 \\
0,802 \\
56 \\
\end{array}$ \\
\hline $\begin{array}{l}\text { Erfolg aus der } \\
\text { Binnen- } \\
\text { perspektive }\end{array}$ & $\begin{array}{r}\mathbf{R} \\
\text { Signifikanz }(2- \\
\text { seitig) } \\
\mathrm{N} \\
\end{array}$ & $\begin{array}{r}0,072 \\
0,605 \\
54 \\
\end{array}$ & $\begin{array}{r}, 415\left(^{(* 亠}\right) \\
0,002 \\
55 \\
\end{array}$ & $\begin{array}{r}0,158 \\
0,255 \\
54 \\
\end{array}$ & 127 & $\begin{array}{r}.368(-) \\
0 \\
127 \\
\end{array}$ & $\begin{array}{r}, 2941^{(\star)} \\
0,001 \\
126 \\
\end{array}$ \\
\hline $\begin{array}{l}\text { Erfolg aus der } \\
\text { Außen- } \\
\text { perspektive }\end{array}$ & $\begin{array}{r}\mathbf{R} \\
\text { Signifikanz }(2- \\
\text { seitig) } \\
\mathrm{N}\end{array}$ & $\begin{array}{r}0,047 \\
0,731 \\
55 \\
\end{array}$ & $\begin{array}{r}.437(+4) \\
0,001 \\
56 \\
\end{array}$ & $\begin{array}{r}, 345\left(^{(*+)}\right. \\
0,01 \\
55 \\
\end{array}$ & $\begin{array}{r}, 368(\text { (-t) } \\
0 \\
127 \\
\end{array}$ & 128 & $\begin{array}{r}, 557(*) \\
0 \\
127 \\
\end{array}$ \\
\hline $\begin{array}{l}\text { Erfolg aus der } \\
\text { Finanz- } \\
\text { perspektive }\end{array}$ & $\begin{array}{r}\mathbf{R} \\
\text { Signifikanz }(2- \\
\text { seitig) } \\
\mathrm{N}\end{array}$ & $\begin{array}{r}0,232 \\
0,088 \\
55\end{array}$ & $\begin{array}{r}, 470(*) \\
0 \\
57\end{array}$ & $\begin{array}{r}0,034 \\
0,802 \\
56\end{array}$ & $\begin{array}{r}, 294 \text { (") }^{\text {* }} \\
0,001 \\
126\end{array}$ & $\begin{array}{r}, 557(+*) \\
0 \\
127\end{array}$ & 129 \\
\hline
\end{tabular}

** Die Korrelation ist auf dem Niveau von 0,01 (2-seitig) signifikant.

- Die Korrelation ist auf dem Niveau von 0,05 (2-seitig) signifikant. 


\section{Liste der zitierten Literatur:}

Anderson, E./Fornell, C./Lehmann, D. (1994): Customer Satisfaction, Productivity, and Profitability: Differences Between Goods and Services. In: Marketing Science, Vol. 16/2, S. 129145.

Anderson, E./Sullivan, M. (1993): The Antecedents and Consequences of Customer Satisfaction for Firms. In: Marketing Science, Vol. 12, S. 125-142.

Anfang, P./Urban, D. (1994): „Vertrauen“- soziologisch betrachtet. Ein Beitrag zur Analyse binärer Interaktionssysteme. In: SISS: Schriftreihe des Instituts für Sozialwissenschaften der Universität Stuttgart. No.1, Stuttgart.

Axelrod, R. (1986): The Evolution of Cooperation. Basic Books, New York.

Backhaus, K./Erichson, B./Plinke, W./Weiber, R. (2000): Multivariate Analysemethoden - Eine anwendungsorientierte Einführung. Springer, Berlin/Heidelberg.

Bacon, F. (1990): Novum Organum. (Übersetzung aus dem Lateinischen von R. Hoffman) Meiner, Hamburg.

Beck, R., Schwarz, G. (2000): Konfliktmanagement. 2. Auflage, Sandmann, Alling.

Becker, W. (1971): Hegels Phänomenologie des Geistes. Kohlhammer, Suttgart.

Benninghaus, H. (2001): Einführung in die sozialwissenschaftliche Datenanalyse - Buch mit CDROM. Oldenburg, München.

Berger, J./Noorderhaven, N. G./Nooteboom, B. (1995): The determinants of supplier dependence: An empirical study. In: Groenewegen, J./Pitelis, C./Sjostrand, S. E. (Hrsg.): On economic institutions: Theory and applications. S. 195-212, Edward Elgar, Aldershot UK.

Bertl, R./Kofler H./Mandl, D. (1992): Die Praxis der neuen Rechnungslegung. Orac, Wien.

Bhide, A./Stevenson, H. H. (1991): Warum rechtschaffend sein, wenn sich Lug und Trug lohnen? In: Harvard Manager, 13. Jahrgang, II Quartal, S. 124-131.

Bierhoff, H. W. (1991): Soziale Motivation kooperativen Verhaltens. In: Wunderer, R. (Hrsg.): Kooperation - Gestaltungsprinzipien und Steuerung der Zusammenarbeit zwischen Organisationseinheiten. Poeschel, Stuttgart.

Boston Consulting Group (BCG) (1970): Experience Curvers as a Planning Tool. A special commentary published by the Boston Consulting Group, Boston.

Bruhn, M. (1996): Qualitätsmanagement für Dienstleistungen. Springer, Berlin.

Bühl, A/Zöfl, P (2000): SPSS Version 10 - Einführung in die moderne Datenanalyse unter Windows. Addison Wesely Verlag, München.

Burger, A. (1995): Jahresabschlussanalyse; Verlag, Eichstätt. 
Buzell, R. D./Gale, B. T./Sultan, R.G. (1975): Market Share - A Key to Profitability. In: Harvard Business Review, Jän.-Feb.

Classen, M. (1983): Aktuelle Probleme strategischer Unternehmensführung und -planung. In: Management Forum, Band 4., S. 47-69.

Converse, J. M./Presser, S. (1986): Survey Questions - Handcrafting the Standardizes Questionnaire. Sage Publications, Berverly Hills.

Dahlstrohm, R./Boyle, B (1994): Behavioural Antecedents to Intrinsic Motivation in Capital Equipment Exchange Relationships. In: Journal of Applied Business Research, Vol. 10, No. 2, S.50-62.

Danner, H. (1998): Methoden geisteswissenschaftlicher Pädagogoik - Einführung in Hermeneutik, Phänomenologie und Dialektik. 4.Aufl. Reinhardt/UTB, München.

Descartes, R. (1960): Von der Methode des richtigen Verstandesgebrauchs und der wissenschaftlichen Forschung. Meiner, Hamburg.

Devereux, G. (1967): Angst und Methode in den Verhaltenswissenschaften. Hanser, München.

Diekmann, A. (2002): Empirische Sozialforschung - Grundlagen, Methoden, Anwendungen. Rowohlt, Hamburg.

Dithey, W. (1974): Der Aufbau der geschichtlichen Welt in den Geisteswissenschaften. Suhrkamp, Frankfurt am Main.

Egger, A./Samer, H. (1994): Der Jahresabschluss nach dem Rechnungslegungsgesetz: Erstellung/Analyse. Linde, Wien.

Engel, Ch. (1999): Vertrauen: ein Versuch. Preprints aus der Max-Planck-Projektgruppe Recht und Gemeinschaftsgüter, Bonn.

Feyerabend P. (2002): Probleme des Empirismus - Teil 1. (Übersetzung aus dem Englischen) Reclam, Stuttgart. Originaltext erschienen in: Colodny, R., G. (Hrsg.): Beyond the Edge of Certainty, Essays in Contemporary Science and Philosophy, Bd. 2, Englewood Cliffs: PrenticeHall, 1965, S.145-260.

Feyerabend, P. (1975): Wider den Methodenzwang - Skizze einer anarchistischen Erkenntnistheorie. Suhrkamp, Frankfurt am Main.

Fink, M. (2002): Osterweiterung der Europäischen Union - Positionen ausgewählter österreichischer Interessensvertretungen. Wiwis. Diplomarbeit, Wirtschaftsuniversität Wien, Wien.

Fink, M./Rößl, D. (2004): Instrumentelles und maximenbasiertes Vertrauen als Erfolgsfaktor von Kooperationen - Zur besonderen Relevanz maximenbasierten Vertrauens in Kooperationen zwischen KMU. In: Meyer, J. A. (Hrsg.): Kooperationen von kleinen und mittleren Unternehmen in Europa. Eul, Lohmar-Köln. 
Fink, M./Rößl, D. (2004): Die Bewertung von Kooperationen und Kooperationspartnern. In: Meyer, J. A. (Hrsg.): Kooperationen von kleinen und mittleren Unternehmen in Europa. Eul, Lohmar-Köln.

Foerster, Heinz von (1993): Wissen und Gewissen - Versuch einer Brücke. Suhrkamp, Frankfurt am Main.

Friedell, E. (1983): Selbstanzeige. Essays ab 1919. Löcker, Wien.

Fröhlich, E./Pichler, J.H. (1988): Werte und Typen mittelständischer Unternehmer. Strategische Erfolgsanalyse: Strategien, Ziele und Werthaltungen erfolgreicher Unternehmer nach „Bereichen“. Duncker\&Humblot, Berlin.

Fuchs, R. (1977): Marktanteils- und Feldanteilsberechnung. In: Behrens, Karl C. (Hrsg.): Handbuch der Marktforschung. Gabler, Wiesbaden.

Gadamer, G. (1990): Wahrheit und Methode. Grundzüge einer philosophischen Hermeneutik. 6.Aufl., Mohr, Tübingen.

Gassenheimer, J. B./Baucus, D. B./Baucus, M. S. (1996): Cooperative Arrangements among Enterpreneurs: An Analysis of Opportunism and Communication in Franchise Structures. In: Journal of Business Research, Vol. 36, S. 67-79.

Gierl, H. (2001): Opportunismus in Geschäftsbeziehungen - Ursachen und Gegenmaßnahmen. In: Der Markt. 41. Jg. Heft 2+3, Nr. 157/158, S.55-65.

Girtler, R. (2001): Methoden der Feldforschung. Böhlau, Wien/Köln/Weimar.

Glasersfeld, Ernst von (1991): Abschied von der Objektivität. In: Watzlawick, P. (Hrsg.): Das Auge des Betrachters. Piper, München.

Goller-Schreckenberg, D. (1999): Grundlagen der Pflegewissenschaft, Seminarunterlage, Universität Osnabrück 1999.

Gräfer, H. (1994): Bilanzanalyse: Eine Einführung mit Aufgaben und Lösungen. 6. Auflage, Verlag neue Wirtschaft-Briefe, Berlin.

Grathoff, R. in: Käsler, D. (Hrg.) (1978): Klassiker des soziologischen Denkens. Beck, München.

Habamas, J. (1973): Erkenntnis und Interesse. Suhrkamp, Frankfurt am Main.

Handwörterbuch der Marktforschung. Gabler, Wiesbaden, S. 643-660.

Hardin, R. (1971): Collective Action an Agreeable n-Prisoners' Dilemma. In: Science. Vol. 165, S. $472-481$.

Hauser, H. (1991): Institutionen zur Unterstützung wirtschaftlicher Kooperation. In: Wunderer, R. (Hrsg.): Kooperation - Gestaltungsprinzipien und Steuerung der Zusammenarbeit zwischen Organisationseinheiten. Stuttgart, S. 107-125. 
Hedley, B. (1977): Strategy and the Business Portfolio. In: Long Range Planning. Feb., S. 9-15.

Hegel, F., W. (1989): Phänomenologie des Geistes. In: Werke - Auf der Grundlage der Werke von 1832-45. Bd. 3, Suhrkamp, Frankfurt am Main.

Helbling, C. (1994): Bilanz und Erfolgsanalyse: Lehrbuch und Nachschlagwerk für die Praxis mit besonderer Berücksichtigung der Darstellung im Jahresabschluss- und Revisionsbericht. 9. Aufl., Verlag Paul Haupt, Zürich.

Hermann, A. (1984): Wie die Wissenschaft ihre Unschuld verlor - Macht und Missbrauch der Forscher. DVA, Stuttgart.

Hitzler, A. (1993): Verstehen: Alltagspraxis und wissenschaftliches Programm. In: Jung, T., Müller-Doohm, S.: „Wirklichkeit“ im Deutungsprozess - Verstehen und Methoden in den Kulturund Sozialwissenschaften. Suhrkamp, Frankfurt am Main.

Hofstätter, L./Mayerhofer, H./ Würdinger, M. (1994): Betriebswirtschaftliche Kennzahlen in der Praxis. Kennzahlenhandbuch. Schriftreihe des Wirtschaftsförderungsinstitutes, Band 245, Wien.

Homburg, C./Rudolph, B. (1995): Wie zufrieden sind Ihre Kunden tatsächlich? In: Harvard Business Manager, Nr. 1, S. 43-50.

Horkheimer, M. (1968): Kritische Theorie. Fischer, Frankfurt.

Horovitz, J./Panak, M. J. (1993): Marktführer durch Service. Lehren aus 50 hervorragenden europäischen Unternehmen. Campus, Frankfurt.

Hume, D. (1967): Eine Untersuchung über den menschlichen Verstand. Reclam, Stuttgart.

John, G. (1984): An Empirical Investigation on Some Antecedents of Opportunism in a Marketing Channel. In: Journal of Marketing Research, Vol. 21, S. 278-289.

Jung, T., Müller-Doohm, S. (1993): >Wirklichkeit< im Deutungsprozess - Verstehen und Methoden in den Kultur- und Sozialwissenschaften. Suhrkamp, Frankfurt am Main.

Kant, I. (1998): Kritik der reinen Vernunft. Meiner, Hamburg.

Kanter, R. M. (1995): Unternehmenspartnerschaften: Langsam zueinander finden. In: Havard Business Manager, 17. Jahrgang, II Quartal, S. 33-44.

Kasper, H. (1990): Die Handhabung des Neuen in organisierten Sozialsystemen. Springer, Berlin - Heidelberg - New York.

Kettel, C. W. (2002): Vertrauen als Organisationsprinzip zwischenmenschlicher Austauschbeziehungen. Institut für Wirtschaftswissenschaften Rheinisch-Westfälische Technische Hochschule Aachen, Lehrstuhl für Unternehmenstheorie, insbesondere Umweltökonomie und industrielles Controlling RWTH Aachen, Arbeitsbericht Nr. 02/03.

Keuth, H. (Hsg.) (1998): Karl Popper - Logik der Forschung, Akademie Verlag, Berlin. 
Kieser, A. (1994): Fremdorganisation, Selbstorganisation und evolutionäres Management. In: Zeitschrift für betriebswirtschaftliche Forschung; Nr. 46, Nr. 3, S. 199-228.

King, G./ Robert, O. K./ Verba, S. (1994): Designing Social Inquiry - Scientific Inference in Qualitative Research. Princeton University Press, Princeton.

Kirchhoff, S./Kuhnt, S./Lipp, P./Schlawin, S. (2001): Der Fragebogen - Datenbasis, Konstruktion und Auswertung. Leske+Budrich Opladen, Wiesbaden.

Kirsch, W. (1983): Fingerspitzengefühl und Hemdsärmligkeit bei der Planung im Mittelstand. In: Kirsch, W./Roventa, P. (Hrsg.): Bausteine eines strategischen Managements: Dialoge zwischen Wissenschaft und Praxis. Berlin - New York, S. 399-422.

Knorr-Cetina, K. (1981): Die Fabrikation von Erkenntnis - Zur Anthropologie der Naturwissenschaft. Suhrkamp, Frankfurt am Main.

Kommission der Europäischen Gemeinschaft (Hrsg) (2001): Bericht der Kommission an den Rat, an das Europäische Parlament, an den Wirtschafts- und Sozialausschuss und an den Ausschuss der Regionen - Ein unternehmerisches Europa schaffen. Die Aktivitäten der Union zur Förderung der kleinen und mittleren Unternehmen (KMU), Brüssel [KOM(2001) 98 endgültig].

Kremsner, G. (1983): Ursachen und Beeinflussung der Mitarbeiterfluktuation. Wiwis. Dipl.Arb., WU-Wien, Wien.

Kuhn, T. (1967): Die Struktur wissenschaftlicher Revolutionen. Suhrkamp, Frankfurt am Main.

Lenz, H. (1990): Moralische Normen und Opportunismus in der neueren Theorie der Unternehmung. In: Schauenberger, B. (Hrsg.): Wirtschaftsethik - Schnittstelle von Ökonomie und Wissenschaftstheorie. Gabler, Wiesbaden.

Lorenz, K. (1973): Die Rückseite des Spiegels. Versuch einer Naturgeschichte menschlichen Erkennens. R. Piper-Verlag, München, Zürich.

Lötschner, F. (1998): Warum ist die Kundenzufriedenheit so wichtig? In: Sonderdruck aus Management \& Qualität, Heft 2, S. 4-10.

Luhmann, N. (1977): Funktion der Religion. Suhrkamp, Frankfurt am Main.

Luhmann, N. (1984): Soziale Systeme - Grundriss einer allgemeinen Theorie. Suhrkamp, Frankfurt am Main.

Luhmann, N. (1989): Vertrauen. Ein Mechanismus der Reduktion sozialer Komplexität. 3. Aufl., Enke, Stuttgart.

Maturana, H. (1988): Reality: the search for objectivity; or the quest for a compelling argument. The Irish Journal of Psychology, Vol. 9 (1).

Mayer, H. O. (2002): Interview und schriftliche Befragung. Oldenbourg, München.

Mellerowicz, K. (1973): Kosten und Kostenrechnung. Band 1, 5. Aufl., de Gruyter, Berlin. 
Meyer, A./Dornach, F. (1998): Das Deutsche Kundenbarometer - Qualität und Zufriedenheit. In: Simon, H./Homburg, C. (Hrsg.): Kundenzufriedenheit. Konzepte - Methoden - Erfahrungen. 3.Aufl., Gabler, Wiesbaden.

Mill, J., S. (1862): A System of Logic - Ratiocinative and Inductive. Hamper, New York.

Mobley, W. H./Hand, H. H./Becker, R. L./Meglino, B. M. (1979): Conceptual and Empirical Analyses of Military Recruit Training Attrition - JAPS/86.

Moore G., E. (1988): Principia Ethica. Prometheus Books, New York.

Müller, M., Halder, A. (1971): Kleines philosophisches Wörterbuch. Herder, Freiburg im Breisgau.

Müller, W./Riesenbeck, H.-J. (1991): Wie aus zufriedenen auch anhängliche Kunden werden. Harvard Manager, Nr. 3, S.67-79.

Mugler, J. (1999a): Betriebswirtschaftslehre der Klein- und Mittelbetriebe. Band 1, Springer, Wien/New York.

Murakami, Y./Rohlen, T. P. (1992): Social-exchange aspects of Japanese political economy: Culture, efficiency and change. In: Kumon, S./Rosorsky, H. (Hrsg.): The political economy of Japan, vol. 3 - Cultural and social dynamics, S. 63-105, Stanford University Press, Stanford.

Muris, T. J. (1981): Opportunistic Behaviour and the Law of Contracts. In: Minnesota Law Review, Vol. 65, Vol. 4, S. 521-590.

Neubauer, F. F. (1980): Das PIMS - Programm und Portfoliomanagement. In: Hahn, D./Taylor, B. (Hrsg.): Strategische Unternehmensplanung - Stand und Entwicklungstendenzen. Würzburg, Wien, S. 135-161.

Nieder, P. (1992): Fluktuation. In: Barath (Hrsg.): Handwörterbuch. o.O.

Nohr, H. (2001): Management der Informationsqualität. Arbeitspapiere Wissensmanagement Nr. 3, Fachhochschule Stuttgart - Studienlehrgang Informationswirtschaft, Stuttgart.

Noorderhaven, N. G. (1995): Trust and Transaction - Towards Transaction Cost Analysis with Differential Behavioural Assumption. In: Tijdschrift voor Economie en Management, Vol. 15, Nr. 1,S. 5-18.

Noorderhaven, N. G. (1996): Opportunism and Trust in Transaction Cost Economics. In: Groenewagen, J. (Hrsg.): Transaction Cost Economics and Beyond, Kluwer Academic Publishers, Boston, S.107-128.

Osterloh, M./Weibel, A. (2000): Ressourcensteuerung in Netzwerken: Eine Tragödie der Allmende? In: Sydow, J./Windeler, A. (Hrsg.): Steuerung von Netzwerken. Konzepte und Praktiken, Opladen, Wiesbaden, S. 88-106.

Ostrom, E. (1998): A Behavioural Approach to the Rational Choice Theory of Collective Action. In: American Political Science Review, Vol. 92, (1), S. 1-22. 
Peirce, C., S. (1976): Schriften zum Pragmatismus und Pragmatizismus. Appel K., O. (Hrsg.), Suhrkamp, Frankfurt am Main.

Pettit, P./Sugden, R. (1989): The Backward Induction Paradox. In: Journal of Philosophy Vol. 86, S. $169-182$.

Piaget, J. (1996): Einführung in die genetische Erkenntnistheorie. Übersetzung von Friedhelm Herborth. Suhrkamp, Frankfurt am Main.

Pleitner, J.H./Rößl, D. (1995): Wirtschaftsförderung unterhalb der Wirtschaftspolitik - der Beitrag zwischenbetrieblicher Kooperation. In: Brandenberg, A. (Hrsg.): Standpunkte zwischen Theorie und Praxis: Handlungsorientierte Problemlösung in Wirtschaft und Gesellschaft. Haupt, Bern/Wien, S. 671-689.

Plötner, O. (1995): Das Vertrauen des Kunden - Relevanz, Aufbau und Steuerung auf industriellen Märkten. Gabler, Wiesbaden.

Popper, K. (1995): Eine Welt der Propensitäten. Tübingen.

Poppo, L. H. (1991): Reputation and Supplier Opportunism in Markets and Firms, Dissertation, University of Pennsylvania.

Provan, K. G./Skinner, S. J. (1989): Interorganizational Dependence and Control an Predictors of Opportunism in Dealer-Supplier Relations. In: Academy of Management Journal, Vol. 32, S. 202-212.

Quartapelle, A. Q./Larsen, G. (1996): Kundenzufriedenheit. Wie Kundentreue im Dienstleistungsbereich die Rentabilität steigert. Springer, Berlin.

Reichelt, F./Sasser, W. (1991): Zero-Migration: Dienstleister im Sog der Qualitätsrevolution. Harvard Manager, Nr. 4, S. 108-116.

Riffner, B/Weidelich, R (2001): Professionelles Lieferantenmanagement - so arbeitet man erfolgreich zusammen. Deutscher Wirtschaftsdienst, Köln.

Ripperger, T. (1998): Ökonomik des Vertrauens: Analyse eines Organisationsprinzips. Mohr Siebeck, Tübingen.

Rohwer, G./Pötter, U. (2001): Grundzüge der sozialwissenschaftlichen Statistik. Juventa, München.

Rößl, D. (1994a): Gestaltung komplexer Austauschbeziehungen - Analyse zwischenbetrieblicher Kooperation. Gabler, Wiesbaden.

Rößl, D. (1994b): Selbstverpflichtung als effiziente Koordinationsform von Austauschbeziehungen und Möglichkeiten ihrer Nutzung im Klein- und Mittelbetrieb. (Herg.) Mugler, J.: Materialien der Abteilung für Gewerbe-, Klein- und Mittelbetriebe an der Wirtschaftsuniversität Wien.

RößI, D. (1996): Selbstverpflichtung als alternative Koordinationsform von komplexen Austauschbeziehungen. In: zfbf, Nr. 48, S. 311-323. 
Rößl, D. (2000): Beziehungsmanagement im Klein- und Mittelbetrieb. Gestaltung dauerhafter Kundenbeziehungen. Service-Fachverlag, Wien.

Rühl, G. (1980): Aspekte optimaler zwischenbetrieblicher Zusammenarbeit im Zulieferwesen. In: Internationales Gewerbearchiv, Nr. 28, Nr.2, S. 80-91.

Sabathil, P. (1977): Fluktuation von Arbeitskräften. Diss., München.

Schmidtchen, D. (2000): Vertrauen und Recht: Eine ökonomische Analyse. Volkswirtschaftliche Reihe, No. 0004, Universität des Saarlandes - Department of Economics, Saarbrücken.

Scholl, A. (2003): Die Befragung - Sozialwissenschaftliche Methode und komunikationswissenschaftliche Anwendung. UVK Verlag, Konstanz.

Schülein, J., A. (2002): Autopoietische Realität und konnotative Theorie - Über Balanceprobleme sozialwissenschaftlichen Erkennens. Velbrück, Weilerswist.

Schülein, J., A. (2002): Wissenschaftstheorie für Einsteiger. WUV Universitätsverlag, Wien 2002.

Schütz, A. Luckmann, T. (1979): Strukturen der Lebenswelt. Band 1, Frankfurt.

Seiffert, H. (1991): Einführung in die Wissenschaftstheorie I. 11. Aufl. Beck, München.

Seiffert, H. (1992): Einführung in die Hermeneutik: die Lehre von der Interpretation in den Fachwissenschaften. A. Franke Verlag, Tübingen.

Selten, R (1978): The Chain Store Paradox. In: Theory and Decision, Vol. 9, S.127-159.

Shleifer, A. (2004): Does Competition Destroy Ethical Behavior? Havard University, Cambridge (MA).

Simmel, G. (1968): Soziologie. Untersuchungen über die Formen der Vergesellschaftung. 5. Aufl., Duncker \& Humbolt, Berlin.

Simon, H./Homburg, C. (1995): Kundenzufriedenheit als strategischer Faktor. In: Simon, H./Homburg, C. (Hrsg.): Kundenzufriedenheit. Gabler, Wiesbaden.

Steiger, W. (1987): Wahrscheinlichkeit von Planungen und Prognosen. Ein Handbuch für Analysen der Treffsicherheit oder Erfolgswahrscheinlichkeit von Planungen, Vorhersagen und Schätzungen. Verlag Industrielle Organisation, Zürich.

Stenz, K. (1973): Die Arbeitnehmerfluktuation, Wien.

Störig, H., J. (1992): Kleine Weltgeschichte der Philosophie. Fischer, Frankfurt am Main.

Sydow, J. (1992): Strategische Netzwerke - Evolution und Organisation. Gabler, Wiesbaden. 
Thiel, U. (Hsg.) (1979): John Locke, Essay über den menschlichen Verstand. Akademie Verlag GmbH, Berlin 1997.

Töpfer, A. (1996): Kundenzufriedenheit: Die Brücke zwischen Kundenerwartung und Kundenbindung. In: Töpfer, A. (Hrsg.): Kundenzufriedenheit messen und steigern. Leuchterhand, Niewied, S. 1-32.

Töpfer, A./Mann, A. (1995): Kundenzufriedenheit als Messlatte für den Erfolg. In: Töpfer, A. (Hrsg.): Kundenzufriedenheit messen und steigern. Leuchterhand, Neuwied.

Ulrich, P. (1997): Integrative Wirtschaftsethik. Haupt, Bern/Wien.

Vico, G. (1979): Liber metaphysicus. Fink, München.

Vonèche, J.J. (1985): Genetic epistemology: Piaget's theory. International Encyclopedia of Education. Vol. 4., Pergamon, Oxford.

Wathne, O. E./ Heide, J.B. (2000): Opportunism in Interfirm Relationships: Forms, Outcomes and Solutions. In: Journal of Marketing, Vol. 64, S. 36-51.

Weber, H. K. (1998): Rentabilität, Produktivität, Liquidität der Unternehmung, Gabler, Wiesbaden.

Weiss, C. (1997): Die betriebswirtschaftliche Aussagekraft von Kennzahlen des Jahresabschlusses exemplarisch untersucht anhand eines Unternehmens der Textilbranche. Wiwis. Diplomarbeit, Wien.

Wenzel, U. (2000): Vom Ursprung zum Prozess. Zur Rekonstruktion des Aristotelischen Kausalitätsverständnisses und seiner Wandlung bis zur Neuzeit. Opladen, o.O..

Wiegand, W. (1994): Rechtsschein und Vertrauen. In: Hof, H./Kummer, H./Weingart, P. (Hrsg.): Recht und Verhalten. Verhaltensgrundlagen des Rechts - zum Beispiel Vertrauen. Baden-Baden, S. 183-198.

Wurche, S. (1991): Vertrauen und ökonomische Rationalität in kooperativen Interorganisationsbeziehungen. In: Wunderer, R. (Hrsg.): Kooperation - Gestaltungprinzipien und Steuerung der Zusammenarbeit zwischen Organisationseinheiten, Stuttgart, S. 144-162.

Wurche, S. (1994): Vertrauen und ökonomische Rationalität in kooperativen Interorganisationsbeziehungen. In: Sydow, J./Windeler, A. (Hrsg.): Management interorganisationaler Beziehungen. Opladen, Wiesbaden, S.142-159.

Zundel, S. (1990): Zur Kritik der Egoismusannahme in der Ökonomie. In: Schauenberger, B. (Hrsg.): Wirtschaftsethik - Schnittstelle von Ökonomie und Wissenschaftstheorie. Gabler, Wiesbaden. 



\section{Weiterführende Literatur:}

Albach, H. (1980): Vertrauen in der ökonomischen Theorie. In: Zeitschrift für die gesamte Staatswissenschaft (ZgS), Jg. 136, Heft 1, S. 2-11.

Atkinson, J.-W. (1957): Motivational Determinants of Risk Taking Behaviour. In: Psychological Review, Vol. 64, S. 359-372.

Beyes, T.B. (2002): Kontingenz und Management. Wiwiss. Diss. Nr. 2643, Universität St. Gallen (HSG), St. Gallen.

Bierhoff, H.W. (1995): Vertrauen in Führungs- und Kooperationsbeziehungen. In: Kieser, A. (Hrsg.): Handwörterbuch der Führung, 2. Aufl., Stuttgart, Sp. 2148-2158.

Bleicher, K. (1991): Normatives Management. Campus, Frankfurt/New York.

Chiles, T.-H./ McMackin, J.F. (1996): Integrating variable Risk Preferences, Trust, and Transaction Cost Economics. In: Academy of Management Review, Vol. 21, S. 73-99.

Dasgupta, P. (1988): Trust as a Commodity. In: Gambetta, D. (Hrsg.): Trust-Making and Breaking Cooperative Relations, Blackwell, Oxford, S. 49-72.

Deutsch, M. (1973): Cooperation and Trust: Some Theoretical Notes, in: Marshall, R.J. (Hrsg.): Nebraska Symposium on Motivation, S. 275-319.

Diesel, E. (1946): Die Macht des Vertrauens, Rinn, München.

Gambetta, D. (1988): Can we Trust Trust?, in: Gambetta, D. (Hrsg.): Trust - Making and Breaking Cooperative Relations, Blackwell, New York, S. 213-237.

Gierl, H. (2000): Opportunismus in langfristigen Geschäftsbeziehungen. In: Schmalenbachs Zeitschrift für betriebswirtschaftliche Forschungen, Bd. 52, S. 107-140.

Grunwald, W. (1995): Wie man Vertrauen erwirbt: Von der Misstrauens- zur Vertrauensorganisation. In: io Management Zeitschrift, Jg. 64, Heft 1/2, S. 73-77.

Heide, J.B./John, G. (1992): Do Norms Matter in Marketing Relationships? In: Journal of Marketing, Vol. 56 (April), S.32-44.

Kramer, R.M./Brewer, M.B./Hanna, B.A. (1996): Collective Trust and Collective Action: The Decision to Trust as a Social Decision. In: Kramer, R.M./Tyler, T.R. (Hrsg.): Trust in Organizations: Frontiers of Theory and Research. Sage Publications, Thousand Oaks, S. 357-389.

Kramer, R.M./Tyler, T.R. (1996): Whither Trust? In: Kramer, R.M./Tyler, T.R. (Hrsg.): Trust in Organizations: Frontiers of Theory and Research, Sage Publications, Thousand Oaks, S. 1-15.

Krystek, U. (1997): Vertrauen als vernachlässigter Erfolgsfaktor der Internationalisierung. In: Krystek, U./Zur, E. (Hrsg.): Internationalisierung: Eine Herausforderung für die Unternehmensführung, Berlin, S. 543-562. 
Loose, A./Sydow, J. (1994): Vertrauen und Ökonomie in Netzwerkbeziehungen Strukturationstheoretische Betrachtung. In: Sydow, J./Windeler, A. (Hrsg.): Management interorganisationaler Beziehungen, Opladen, Wiesbaden, S. 160-193.

Luhmann, N. (1988): Familiarity, Confidence, Trust: Problems and Alternatives. In: Gambetta, D. (Hrsg.): Trust- Marking and Breaking Cooperative Relations, Blackwell, New York, S. 94-107.

Manz, W. (1980): Gefangen im Gefangenendilemma? Zur Sozialpsychologie experimenteller Spiele. In: Bungard W. (Hrsg.): Die „gute“ Versuchsperson denkt nicht - Artefakte in der Sozialpsychologie, Urban\&Schwarzenberg, München, S. 145-166.

Mishra, A.K. (1996): Organizational Responses to Crises: The Centrality of Trust. In: Kramer, R.M./Tyler, T.R. (Hrsg.): Trust in Organisations - Frontiers of Theory and Research, Sage Publications, Thousand Oaks, S. 261-287.

Nieder, P. (1997): Erfolg durch Vertrauen - Abschied vom Management des Misstrauens. Gabler, Wiesbaden.

Nooteboom, B./Berger, H./Noorderhaven, H.G. (1997): Effects of trust and Governance on Relational. In: Academy of Management Journal, Vol. 40, S. 308-338, Boston, S. 105-128.

Petermann, F. (1992): Psychologie des Vertrauens, 2. A., Quintessenz-Verlag, München.

Pieper, J. (2000): Vertrauen in Wertschöpfungspartnerschaften: eine Analyse aus Sicht der neuen Instutitionenökonomie, Gabler, Wiesbaden.

Platzköster, M. (1989): Vertrauen: Theorie und Analyse interpersoneller, politischer und betrieblicher Implikationen, Beleke, Essen.

Rößl, D. (1992): Die Funktion und die Bildung von Vertrauen in der unternehmerischen Partnerschaft. In: Betriebswirtschaftliches Institut für empirische Gründungs- und Organisationsforschung e. V. (Hrsg.): Partnerschaftsmanagement in unternehmerischen Partnerschaften. Dortmund, S. 54-71.

Rotter, J,B. (1971): Generalised Expectancies for Interpersonal Trust. In: American Psychologist, Vol. 26, S. 443-452.

Rotter, J.B. (1967): A new scale for the measurement of Interpersonal Trust. In: American Psychologist, Vol. 35, S.651-665.

Siegenthaler, H. (1987): vertrauen - Ein Schlüsselbegriff bei Keynes und Schumpeter. In: Timmermann, M. (Hrsg.): Die ökonomischen Lehren von Marx, Keynes und Schumpeter, Stuttgart, S. 80-99.

Walgenbach, P. (2000): Das Konzept der Vertrauensorganisation - Eine theoriegeleitete Betrachtung. In: Die Betriebswirtschaft, Jg. 60, Heft. 6, S. 707-720. 


\section{Forschungsergebnisse der Wirtschaftsuniversität Wien}

Herausgeber: Wirtschaftsuniversität Wien vertreten durch a.o. Univ. Prof. Dr. Barbara Sporn

Band 1 Stefan Felder: Frequenzallokation in der Telekommunikation. Ökonomische Analyse der Vergabe von Frequenzen unter besonderer Berücksichtigung der UMTS-Auktionen. 2004.

Band 2 Thomas Haller: Marketing im liberalisierten Strommarkt. Kommunikation und Produktplanung im Privatkundenmarkt. 2005.

Band 3 Alexander Stremitzer: Agency Theory: Methodology, Analysis. A Structured Approach to Writing Contracts. 2005.

Band 4 Günther Sedlacek: Analyse der Studiendauer und des Studienabbruch-Risikos. Unter Verwendung der statistischen Methoden der Ereignisanalyse. 2004.

Band 5 Monika Knassmüller: Unternehmensleitbilder im Vergleich. Sinn- und Bedeutungsrahmen deutschsprachiger Unternehmensleitbilder - Versuch einer empirischen (Re-)Konstruktion. 2005.

Band 6 Matthias Fink: Erfolgsfaktor Selbstverpflichtung bei vertrauensbasierten Kooperationen. Mit einem empirischen Befund. 2005.

www.peterlang.de 


\section{Mittelstandsforschung}

\section{Beiträge zu Strategien, Finanzen und zur Besteuerung von KMU}

Frankfurt am Main, Berlin, Bern, Bruxelles, New York, Oxford, Wien, 2004. 230 S., zahlr. Tab., 2 Graf.

ISBN 3-631-51930-3 · br. € 42.50*

Das Buch enthält 14 Beiträge zu aktuellen Fragestellungen der Mittelstandsforschung. Dabei liefern kleine und mittlere Unternehmen (KMU) den einheitlichen Bezugsrahmen für sämtliche Beiträge. Dargestellt und analysiert werden Einzelaspekte, die den Themenschwerpunkten Strategisches Management, Finanzierung, Recht sowie Steuern und Rechnungslegung zugeordnet werden können. Im Einzelnen umfassen die Beiträge folgende Inhalte: Kooperationen in der Biotechnologiebranche, gesellschaftsrechtliche Gestaltung der Netzwerke virtueller Unternehmen mit Beispielen von KMU in der Biotechnologiebranche, die Bereitstellung von ASP-Lösungen für KMU, die Bedeutung der Kundenzufriedenheit mittelständischer Unternehmen aus Bankensicht, Technologietransfer als Innovationsmotor für den Mittelstand, Entrepreneurship am Beispiel eines konkreten Unternehmens, Nachfolgeproblematik im Mittelstand und Management Buy-out als Lösungsansatz, Finanzierung eines sustainable Entrepreneurs, Analysen zur Notwendigkeit eines Rating sowie der Inanspruchnahme ratingbegleitender Unterstützung bei KMU-Finanzierungen, Anmerkungen zur Unternehmensbewertung im Falle eines personenbezogenen Unternehmens, Buchwertabfindungsklauseln im Personengesellschaftskonzern, die Erfassung verdeckter Gewinnausschüttung nach der so genannten Teil-betragsrechnung und die Auswirkungen des BFH-Urteils vom 6. Juni 2002 auf die Umsatzbesteuerung von GmbH \& Co.KG.

Aus dem Inhalt. Strategisches Management · Finanzierung · Steuern und Rechnungslegung $\cdot$ Recht

Frankfurt am Main - Berlin - Bern - Bruxelles - New York · Oxford · Wien

Auslieferung: Verlag Peter Lang AG

Moosstr. 1, $\mathrm{CH}-2542$ Pieterlen

Telefax 0041 (0) 32 / 3761727

*inklusive der in Deutschland gültigen Mehrwertsteuer

Preisänderungen vorbehalten

Homepage http://umw.peterlang.de 